\title{
Regulation of Skeletal Muscle Carbohydrate Metabolism During Mammalian Hibernation
}

\author{
By: \\ Ryan Andrew Vincent Bell
}

A Thesis Submitted to the Faculty of Graduate and Post Doctoral Affairs in partial

fulfillment of the requirements for the degree of

\author{
Doctor of Philosophy \\ in \\ Chemistry \\ Carleton University \\ Ottawa, Ontario
}

(C) 2014

Ryan Andrew Vincent Bell 


\begin{abstract}
Thirteen-lined (Ictidomys tridecemlineatus) and Richardson's (Urocitellus richardsonii) ground squirrels survive harsh winter conditions by entering hibernation, spending the majority of their time in a state of torpor, where metabolic functions are both strongly suppressed and reprioritized to ensure long term survival. This thesis analyzed biochemical controls on carbohydrate metabolism during hibernation by characterizing the regulation of crucial enzymes of the glycolytic and gluconeogenic pathways: glyceraldehyde-3-phosphate dehydrogenase (GAPDH), pyruvate kinase (PK), and fructose-1,6-bisphosphatase (FBPase). Important signal transduction enzymes regulating carbohydrate metabolism were also evaluated: protein phosphatase $2 \mathrm{~A}$ (PP2A) and glycogen synthase kinase 3 (GSK3). The state of glycolysis in ground squirrel skeletal muscle was assessed by characterizing the bifunctional enzyme GAPDH and the terminal enzyme PK. Results showed that muscle GAPDH and PK activities were substantially suppressed during torpor. PK suppression was linked to reversible serine/threonine phosphorylation. GAPDH regulation was more complex with activity potentially mediated by one or more posttranslational modifications including acetylation, methylation and phosphorylation, as identified through mass spectrometry and Western blot analyses. The gluconeogenic state of muscle was assessed by characterizing FBPase as well as GAPDH operation in its gluconeogenic direction. In both cases, results indicated significant reductions in gluconeogenic function during torpor. Suppressed FBPase activity (i.e. decreased $V_{\max }$, increased $K_{m} F 1,6 P_{2}$ ) was linked with an increase in FBPase phosphorylation and allosteric controls by AMP and $\mathrm{F} 2,6 \mathrm{P}_{2}$. Analysis of PP2A catalytic subunit showed that elevated phosphorylation at
\end{abstract}


tyrosine307 accompanied a significant increase in $\mathrm{K}_{\mathrm{m}}$ peptide, indicating reduced activity of PP2A during torpor. This was corroborated by computational analysis of tyrosine307 phosphorylation effects on substrate binding. Skeletal muscle GSK3 activity also decreased during torpor associated with enhanced GSK3 phosphorylation at serine9. However, the principal substrate of GSK3, glycogen synthase, showed increased phosphorylation suggesting that a different protein kinase was responsible for its control during torpor. Taken together these studies suggest that skeletal muscle glycolysis and gluconeogenesis are suppressed during ground squirrel torpor via posttranslational modification and regulation of key enzymes. Reversible controls over glycolytic and gluconeogenic enzymes would allow for the quick reactivation of muscle metabolism to support shivering thermogenesis and a return to normal euthermic function during arousal. 


\section{Acknowledgements}

There is an exceedingly long list of people who I want to thank for, not only helping me achieve the success I've had in the Storey lab, but for making it such an amazing few years. I guess I'll start with those in the Storey lab whom have made my time here so enjoyable. So, a huge thanks goes out to Ben, Oscar, and Jacques whom made 'work' a blast during my Master's years. A special thanks goes to Mike Wu for the constant friendship throughout my years at Carleton and the continuous talk of all things sports/fantasy/Yankees. A special thanks also goes out to Neal Dawson who was my partner in crime for my $\mathrm{PhD}$ years in the lab. If not for Neal I would have shouldered the blame for many a screw up all by myself.

I also need to thank another friend who brought me to this lab during my undergraduate years and has always had my best interest in mind since we were kids. Thank you Judeh for making my Master's years seem like one big continuous summer vacation. Thank you also for all the support throughout my $\mathrm{PhD}$; there truly is no better friend.

A very special thanks needs to go out to Jan and Ken Storey. Jan, thank you so much for the endless help with my writing and my research. A huge thanks goes to Ken for being my science role model and guiding me through the minefield that is scientific research and enzymology in particular. Without both of your help I would not have been able to achieve my $\mathrm{PhD}$.

Of course a big thanks goes out to my family. Mom and Dad thank you for your constant support and encouragement throughout all my years in school. Thanks to my 
brother, Matt, for your endless support and for being an amazing role model throughout my life. There is no better friend and no better brother.

Lastly, I want to thank my wife and Jacob. Mel, thank you for all the love and support you have given me since the day I met you. You gave meaning to my life beyond my work, and made me realize that the thing that is most important to me is family. I love you with all my heart. Jacob, your arrival was certainly the highlight of my $\mathrm{PhD}$ and the highlight of my life. You mean absolutely everything to your Mom and I, and the best parts of my day were returning home to see you. I love you with all my heart. 


\section{Table of Contents}

Title Page

Abstract

ii

Acknowledgements

iv

Table of Contents

vi

List of Abbreviations

vii

List of Figures

ix

List of Tables

xi

List of Appendices

xii

Chapter 1 General Introduction

1

Chapter 2 Purification and Properties of Glyceraldehyde-3-phosphate

15

Dehydrogenase from the Skeletal Muscle of the Hibernating

Ground Squirrel, Ictidomys tridecemlineatus

Chapter 3 Regulation of skeletal muscle pyruvate kinase from the

hibernating ground squirrel, Urocitellus richardsonii.

Chapter $4 \quad$ Regulation of skeletal muscle fructose-1,6-bisphosphatase

83

from the hibernating ground squirrel, Ictidomys

tridecemlineatus

Chapter 5 Regulation of Protein Phosphatase 2A by Reversible

Phosphorylation during 13-lined Ground Squirrel Torpor

Chapter 6 Regulation of skeletal muscle glycogen synthase kinase-3

from the hibernating thirteen-lined ground squirrel.

Chapter 7 General Discussion

References

Appendices 


\section{List of Abbreviations}

AAPH

ACR

AMPK

APS

AMP

ADP

ATP

1,3BGP

CaMK

CAMP

cGMP

CM

DEAE

DSF

DTT

ECL

EDTA

EGTA

ESI-TOF QUAD MS/MS

FBPase

F1,6P2

F2,6P2

GAP

GAPDH

GnHCl

$\beta$-GP

GS

GSK3

HEPES

HPLC

HSL

I50

Ka

Km

LDH 2,2-azobis(2-amidinopropane dihydrochloride)

Active in the cold room

AMP-activated protein kinase

Ammonium persulphate

Adenosine monophosphate

Adenosine diphosphate

Adenosine triphosphate

1,3-bisphosphoglycerate

Calcium/calmodulin activated protein kinase

3'-5'-cyclic adenosine monophosphate

3'-5'-cyclic guanosine monophosphate

Carboxymethyl

Diethylaminoethyl Sephadex

Differential Scanning Fluorimetry

Dithiothreitol

Enhanced chemiluminescence

Ethylenediamine tetraacetic acid

Ethylene glycol tetraacetic acid

Electrospray ionization-time of flight quadrupole tandem mass spectrometer

Fructose-1,6-bisphosphatase

Fructose-1,6-bisphosphate

Fructose-2,6-bisphosphate

Glyceraldehyde-3phosphate

Glyceraldehyde-3-phosphate dehydrogenase

Guanidine hydrochloride

Beta-glycerophosphate

Glycogen synthase

Glycogen synthase kinase 3

4-(2-hydroxyethyl)-1-piperazineethanesulfonic acid

High performance liquid chromatography

Hormone-sensitive lipase

Concentration of inhibitor that lowers enzyme velocity by $50 \%$

Activator concentration that gives $1 / 2$ maximal enzyme activation

Substrate concentration at $1 / 2$ maximal enzyme velocity

Lactate dehydrogenase 


\begin{tabular}{ll} 
LH & Late hibernation \\
MES & 2-ethanesulfonic acid \\
MPA & Microplate Analysis Program \\
NAD & Nicotinamide adenine dinucleotide (oxidized) \\
NADH & Nicotinamide adenine dinucleotide (reduced) \\
NADP & Nicotinamide adenine dinucleotide phosphate (oxidized) \\
PAGE & Polyacrylamide gel electrophoresis \\
PCR & Polymerase chain reaction \\
PEP & Phosphoenolpyruvate \\
PEPCK & Phosphoenolpyruvate carboxykinase \\
PK & Pyruvate kinase \\
PKA & Protein kinase A \\
PKC & Protein kinase C \\
PMA & phorbol 12-myristate 13-acetate \\
PMSF & Phenylmethylsulphonyl fluoride \\
PP2Ac & Protein phosphatase 2A catalytic subunit \\
PTM & Posttranslational modification \\
PTP & Protein tyrosine phosphatase \\
PVDF & Polyvinylidiene difluoride \\
3PG & 3-phosphoglycerate \\
RQ & Respiratory quotient \\
SDS & Sodium dodecyl sulphate \\
TBS & Tris-buffered saline \\
TBST & Tris-buffered saline plus Tween-20 \\
TEMED & Tetramethylethylenediamine \\
Tb & Body temperature \\
Tm & Protein melting temperature \\
Tris & Tris(hydroxymethyl)aminomethane \\
Vmax & Maximal enzyme velocity \\
WAT & White adipose tissue \\
\hline
\end{tabular}




\section{List of Figures}

Figure 1.1 The body temperature of golden-mantled ground squirrel 14 (Ictidomys tridecemlineatus) monitored over one year.

Figure 2.1 Purified ACR skeletal muscle GAPDH. 44

Figure 2.2 Heat-induced inactivation of ACR and LH skeletal muscle 45 GAPDH.

Figure 2.3 Phosphorylation state of skeletal muscle ACR and LH 46 GAPDH

Figure 2.4 Posttranslational modification of ACR and LH skeletal muscle 47 GAPDH

Figure 2.5 Effect of in vitro incubations that stimulate protein kinases or 48 protein phosphatases on GAPDH activity.

Figure 2.6 Cytoplasmic and nuclear expression of skeletal muscle

GAPDH as determined by immunoblotting.

Figure 3.1 Purified euthermic PK from the skeletal muscle of $U$. 77 richardsonii.

Figure 3.2 $\mathrm{pH}$ curve for euthermic and torpid PK from the skeletal 78 muscle of $U$. richardsonii.

Figure 3.3 Thermal stability of euthermic and torpid PK from the skeletal 79 muscle of $U$. richardsonii.

Figure 3.4 Phosphorylation state of euthermic and torpid PK from the $\quad 80$ skeletal muscle of $U$. richardsonii.

Figure 3.5 Effect of alkaline phosphatase incubations on the $K_{m}$ ADP of 81 PK.

Figure 3.6 Analysis of posttranslational modification to skeletal muscle

PK from $U$. richardsonii as assessed through Western blots.

Figure 4.1 Partial purification of skeletal muscle FBPase from the ACR 98 condition.

Figure 4.2 Velocity versus $\mathrm{F} 1,6 \mathrm{P}_{2}$ substrate concentration curves for partially purified FBPase derived from ACR and LH muscle samples.

Figure 4.3 Deinhibition of muscle FBPase from ACR and LH conditions 100 with $10 \mathrm{mM} \mathrm{Mg}$ :citrate or $10 \mathrm{mM}$ histidine.

Figure 4.4 Inhibition of muscle FBPase by urea. 101

Figure 4.5 Muscle FBPase phosphorylation state. 102

Figure 4.6 Posttranslational acetylation of muscle FBPase. 103

$\begin{array}{lll}\text { Figure 4.7 The effect of in vitro incubations to stimulate endogenous } & 104\end{array}$ protein kinases and commercial protein phosphatase on $\mathrm{K}_{\mathrm{m}}$ F1,6 $\mathrm{P}_{2}$ for ACR and LH FBPase. 
Figure 5.1 Representative elution profile for ACR PP2Ac off of a DEAE- 123 Sephadex column.

Figure 5.2 Purification of PP2Ac from the skeletal muscle of ACR 13- 124 lined ground squirrels.

Figure 5.3 Relative levels of 13-lined ground squirrel PP2Ac and 125 phospho-PP2Ac in ACR and LH skeletal muscle preparations.

Figure 5.4 Posttranslational modifications of PP2Ac from skeletal 126 muscle of ACR and LH conditions: acetylation, and lysine and arginine methylation.

$\begin{array}{lll}\text { Figure 5.5 Peptide docking to the dephosphorylated PP2Ac homology } & 127\end{array}$ model produced from the 13-lined ground squirrel amino acid sequence.

Figure 5.6 Peptide docking to phosphorylated Y307-PP2Ac homology model produced from the 13-lined ground squirrel amino acid sequence.

Figure 6.1 Purified GSK3 from the skeletal muscle of ACR 13-lined ground squirrels.

Figure 6.2 ATP standard curves for Kinase-Glo Plus luminescent kinase assay platform.

Figure 6.3 $\mathrm{K}_{\mathrm{m}}$ peptide for GSK3 derived from the skeletal muscle of 13- 146 lined ground squirrels.

Figure 6.4 GSK3 $\beta$ and phospho-GSK3 $\beta$ (Ser9) expression in 13-lined ground squirrel skeletal muscle samples from active (ACR) versus torpid (LH) animals.

Figure 6.5 The levels of total GS and phospho(ser641/645)-GS expression in 13-lined ground squirrel skeletal muscle samples from active (ACR) versus torpid (LH) animals.

Figure 7.1 Enzymes of carbohydrate metabolism. 


\section{List of Tables}

Page

Table 2.1 Purification scheme for 13-lined ground squirrel ACR GAPDH. 40

Table 2.2 Kinetic analysis of ACR and LH GAPDH in the GAP-oxidizing 41 and $1,3 \mathrm{BPG}$-reducing directions at $5^{\circ} \mathrm{C}$ and $36^{\circ} \mathrm{C}$.

Table 2.3 Physical properties of skeletal muscle GAPDH from euthermic 42 and torpid 13-lined ground squirrels.

Table 2.4 The peptides detected by mass spectrometry in ground squirrel 43 muscle GAPDH from ACR versus LH conditions.

Table 3.1 Purification scheme for skeletal muscle PK from euthermic 73 ground squirrels, U. richardsonii.

Table 3.2 Kinetic properties of purified skeletal muscle PK from 74 euthermic and torpid $U$. richardsonii

Table 3.3 Effects of various metabolites on purified euthermic and torpid 75 U. richardsonii skeletal muscle PK.

Table 3.4 The effect of chemical denaturants, urea and guanidine 76 hydrochloride $(\mathrm{GnHCl})$, on the activity of purified euthermic and torpid $S$. richardsonii skeletal muscle PK.

Table 4.1 Partial purification of FBPase from 13-lined ground squirrel 96 ACR muscle.

Table 4.2 Kinetic comparison of 13-lined ground squirrel skeletal muscle 97 FBPase from ACR and LH conditions.

Table 5.1 Purification of PP2Ac from skeletal muscle of ACR 13-lined 121 ground squirrels.

Table 5.2 Kinetic properties of purified PP2Ac from skeletal muscle of 122 13-lined ground squirrels from ACR and LH conditions.

Table 6.1 ACR and LH 13-lined ground squirrel skeletal muscle GSK3 143 kinetics 


\section{List of Appendices}

Page

Appendix I Supplementary Information from Thesis

182

Appendix II $\quad$ List of Publications

192

Appendix III Communications at Scientific Meetings

195 
CHAPTER 1

General Introduction 


\section{Mammalian Hibernation}

Seasonal fluctuations in environmental temperature and resource availability are obstacles encountered by nearly all animals that make their home in the northern latitudes. Indeed, the persistence of the extreme winter climate requires organisms to adopt one of only a few strategies to survive: migrate, subsist on the resources still available while seeking shelter, or seek shelter and become dormant. The latter case is the strategy adopted by few species and is likely the most extreme example of physiological and biochemical plasticity seen in mammals.

The adaptive advantage of entering into a state of aerobic dormancy is in eliminating the need to maintain a body temperature around $37^{\circ} \mathrm{C}$ in cold weather, and thus reducing energy needs for those mammals that are typically high-temperature endotherms. This is particularly important for small mammals that have a high surface area to volume ratio, as heat will be lost quickly in frigid conditions thus necessitating higher rates of metabolism and a corresponding increase in food intake to maintain this metabolic rate. Some mammals that are susceptible to this problem have evolved mechanisms, such as daily torpor or extended torpor bouts, to reduce energy needs and survive until a more moderate climate emerges. Typically these torpor bouts occur in shelters termed hibernacula, which are usually underground burrows, caves or mines that not only protect from the extreme cold and wind, but also protect against potential predation. The temperature within a typical hibernaculum stays slightly above $0^{\circ} \mathrm{C}$ even as outside temperatures plummet to well below freezing (Geiser, 1988).

Mammalian hibernation consists of relatively long periods of torpor, ranging between a few days and 5 weeks, interspersed with periods of arousal usually lasting 
anywhere from 12-24 h (Fig. 1.1). During torpor, the metabolic rate of these animals falls by greater than $95 \%$, with a subsequent decrease in body temperature $\left(T_{b}\right)$ to near ambient temperature (Geiser, 2004). During these periods most physiological functions are slowed or completely arrested. For instance, mammalian heart rate usually drops from the usual 200-300 beats/min to 3-5 beats/min during torpor (Zatman, 1984), respiration rates drop precipitously from 100-200 breaths/min to 4-6 breaths/min (McArthur and Milsom, 1991), renal functioning virtually ceases (Moy, 1971; Zatman, 1984), and even immune function is greatly reduced during torpor (Prendergast et al., 2002). Interrupting these dormant states are short arousal periods where metabolic rate, $\mathrm{T}_{\mathrm{b}}$, and essentially normal physiologic functioning resumes at euthermic levels. These interbout arousals are absolutely necessary for survival throughout the hibernation season, although their function is somewhat debated. Current suggestions include the need to refresh memory circuits through sleep (i.e. torpor is not a sleep state), or a period where the immune system is renewed and important processes are reset (Daan et al., 1991; Larkin et al., 2002; Humphries et al., 2003; Bouma et al., 2010; Levesque and Tattersall, 2010; Zivadinovic et al., 2005).

Such major alterations in metabolic rate between torpid and aroused conditions requires extensive restructuring of the cellular landscape, and a readjustment of ATPproducing and ATP-utilizing processes. For instance, during torpor there is typically a suppression of energy-expensive processes such as transcription, translation, ion transport, protein degradation, and cell division (reviewed in Storey, 2010). These processes are known to consume the bulk of the energy available to cells, and thus their suppression confers major energy savings that allow hibernators to maintain viability 
over the long months of winter. With energy being the critical currency during torpor, a particular emphasis needs to be placed on the regulation of fuel metabolism during hibernation.

\section{Fuel Metabolism}

Pre-hibernation

The hibernation cycle consists of many metabolic changes as the animal transitions into and out of torpor, and alterations to fuel metabolism is an important aspect of this. Fuel metabolism changes begin during the pre-hibernation period with the caching of food inside burrows for some hibernating species, or, as is the case for some ground squirrels, partaking in hyperphagia in the summer and fall months prior to the hibernation season (cited in McKeever 1964; Davis, 1976). Hyperphagia, which is a period of intense eating, dramatically increases the fat stored in white adipose tissue (WAT); increasing body mass by approximately $50 \%$ prior to the hibernation season. The physiological mechanisms that allow for such a drastic build-up of WAT are not completely understood, but some pieces of the puzzle have been put into place.

Pre-hibernatory hormonal changes are responsible for allowing the accumulation of triglycerides in the WAT. As the overeating mammal ingests food during the summer/fall months, the pancreas secretes insulin into the blood, which is a key regulator of carbohydrate and fat metabolism. Although insulin affects several metabolic pathways, particularly important to the build-up of fat reserves is the activation of triglyceride synthesis within WAT. Specifically, insulin binds to receptors on adipocytes leading to two major effects: (1) protein phosphatase 2A becomes activated and dephosphorylates active hormone-sensitive lipase (HSL), and (2) phosphodiesterases 
become active and degrade cAMP, which is a necessary second messenger for cAMP dependent protein kinase (PKA) activation and the phosphorylation of HSL (Stralfors and Honner, 1989). The decreased presence of phosphorylated HSL results in decreased lipolysis and the reduced concomitant release of fatty acids, allowing for the accumulation of triglycerides in the WAT.

In addition to insulin-mediated HSL suppression, there are several lipogenic enzymes that become activated during pre-hibernation. For instance, fatty acid synthase and diacylglycerol acyltransferase enzyme activities are increased during the summer when preparatory lipogenesis is underway (Wang et al., 1997). These changes in WAT cellular biochemistry allow for the build-up of triglycerides that are essential for winter survival.

In most situations, the accumulation of fat in the WAT initiates the synthesis and release of the hormone leptin from adipocytes as part of a negative feedback loop to cause satiation. More specifically, leptin acts at the hypothalamus to decrease appetite and increase metabolic rate. Within most mammalian systems leptin secretion is highly correlated with an increase in adiposity, however, hibernators that have adopted hyperphagia as part of their survival mechanism display some disconnect between leptin signaling and adiposity. Evidence suggests that leptin secretion increases prior to a significant increase in adiposity during pre-hibernation, and that late in pre-hibernation when adiposity is maximal, plasma leptin and leptin secretion appears to decrease (Kronfeld-Schor et al., 2000). There is also evidence that indicates that leptin sensitivity during pre-hibernation may decrease with only high levels of the hormone being able to inhibit hyperphagia (Ormseth et al., 1996). While the exact mechanism by which 
hyperphagia-adopting hibernators can circumvent the leptin system is not completely understood, it is clear that it is important for mammalian preparation for hibernation. While hyperphagia generates a fat depot that will be able to sustain a mammal during its hibernating cycle, under normal circumstances these reserves would become useless at the body temperatures adopted during torpor. This is because in order to mobilize triglyceride-derived fatty acids for use throughout the body, the lipids must be in a fluid state. Typical mammalian body fat has a melting temperature of $25^{\circ} \mathrm{C}$ and would be solid at $0-5^{\circ} \mathrm{C}$, which is typically reached in deep torpor (Wang, 1979). Lipid fluidity is largely dependent upon the degree of unsaturation of the fatty acids in the lipid stores, with increased unsaturation providing greater lipid fluidity. Hence, by eating a diet rich in polyunsaturated fatty acids (PUFAs) (particularly linoleic acid and $\alpha$-linolenic acid) during the hyperphagia stage, the fluidity of adipose depots is adjusted to allow fuel mobilization across the wide range of body temperatures that are encountered during torpor-arousal cycles (Harlow and Frank, 2001; Frank et al., 2008).

While the build-up of fat stores and the associated physiological changes are important during prehibernation, it is also important to consider the state of the other main fuels during this period. For instance, the main carbohydrate store, glycogen, remains relatively constant in both liver and skeletal muscle tissue during the active portions of the ground squirrel life cycle. Similarly, plasma glucose levels remain constant during this period (Galster and Morrison, 1975). Although glucose/glycogen does not appear to be accumulated and excessively stored as seen with lipids, carbohydrate oxidation does account for the majority of energy produced during the prehibernation periods. Evidence for this originates from measurements of the respiratory 
quotient (RQ), which is the ratio of the volume of carbon dioxide produced to the volume of oxygen consumed by a given animal. During the prehibernation period it has been shown that RQ values are near 1 indicating that carbohydrates are the major fuel being oxidized by hibernators during the spring, summer and fall, whereas RQ values drop closer to 0.7 during hibernation which is indicative of lipid oxidation (cited in Carey et al., 2003).

Lastly, the contribution of protein catabolism to energy production during the prehibernation period should be considered. Given that the RQ value nears 1 and that protein breakdown for energy is generally unfavourable, it is expected that the use of amino acids for energy is limited pre-dormancy.

\section{Torpor}

As the pre-hibernatory period recedes and winter conditions ensue, the necessary resources to maintain a normal body temperature become limited and hibernating species begin to cycle through shallow torpor bouts. During this period, biochemical changes are made to favour carbohydrate sparing and lipolysis in most tissues (RQ shifts from 1 to 0.7-0.8; Buck and Barnes, 2000). This clear shift in metabolic fuel usage towards lipid oxidation necessitates significant changes in cellular biochemistry that begin with alterations in circulating hormones. For instance, since feeding has stopped, plasma insulin levels decrease during the hibernation cycle and are at their lowest at mid-winter (Bauman et al., 1987; Carey et al., 2003). With decreased circulating insulin and glucose, it is expected that the counteracting hormone, glucagon, would be released during torpor. Evidence from ground squirrels, however, suggests that glucagon levels remain fairly constant during torpor as compared euthermic controls (Bauman et al., 
1987). Although little increase was seen, the decrease in insulin levels combined with steadied glucagon levels likely means glucagon dominates in the blood. Evidence for this is provided by the numerous metabolic adjustments that occur during torpor, which are typically observed during times of elevated glucagon levels. Mainly, this hormonal state acts to reduce carbohydrate catabolism and initiate tissue lipid oxidation.

The shift in fuel usage from featuring carbohydrates to fats begins with the WAT. This tissue is the storage location for the fatty acids that will be needed to sustain cellular function in a variety of tissues. The substantial levels of circulating glucagon act on the WAT to activate PKA, which in turn phosphorylates and activates HSL for the conversion of triglycerides into free fatty acids. Interestingly, studies on the hibernating jerboa suggest the WAT becomes more sensitive to glucagon during torpor, which may amplify the effect on HSL activation (Moreau-Hamsany et al., 1988). In addition to altered HSL activity, a concomitant decrease in the activity of triglycerol synthesizing enzymes also occurs during mid-winter hibernation in WAT as compared to the prehibernatory and summer periods (Mostafa et al., 1993; Wang et al., 1997). This combination leads to a significant efflux of fatty acids into the blood stream facilitating the reorganization of fuel metabolism within other tissues.

It is important to consider the fact that fatty acids released into the blood have a low solubility, which is exasperated by the low body temperature experienced during torpor. This clearly presents a problem that is typically overcome by the binding of fatty acids to serum albumin, a very abundant protein in the blood, which increases fatty acid solubility dramatically. Early studies did suggest that serum albumin concentrations do increase during mammalian hibernation (cited in Srere et al., 1992; Galster and Morrison, 
1966), likely to facilitate this significant influx of fatty acids into the blood stream. Once bound, serum albumin transports fatty acids to the tissues where they are released and pass across the cell membrane by passive diffusion (Guo et al., 2006).

While circulating glucagon initiates the release of fatty acids from WAT, it also prepares many other tissues for accepting and utilizing fatty acids for energy. Glucagon binds to ubiquitous glucagon receptors and begins a cascade that eventually results in the activation of PKA. This protein kinase, among other things, phosphorylates cAMP response element binding (CREB) protein, which is responsible for mediating the expression of various proteins involved in lipid catabolism. Indeed, studies in several hibernating species have shown that proteins under CREB control such as peroxisome proliferator-activated receptor gamma (PPAR-gamma) and the PPAR coactivator-1 (PGC-1) are elevated in several tissues during torpor (Eddy and Storey, 2003, Eddy et al., 2005). Furthermore, the expression of fatty acid binding proteins (FABPs), which are controlled by PPARs, are elevated in several hibernating species (Hittel and Storey, 2001; Eddy and Storey, 2003; Epperson et al., 2004). These proteins are critical to the transport of lipids through the cytoplasm to the outer mitochondrial membrane where the fats are further processed for entry into the mitochondria and then catabolized for energy.

Directly affecting fuel metabolism in the torpid animal is the drop in insulin levels experienced due to the lack of feeding that occurs during dormancy (Palumbo et al., 1983; Bauman et al., 1987; Carey et al., 2003). It is expected that decreased circulating insulin will decrease signaling through the phosphoinositide-3-kinase (PI3K) and Akt cascade that is normally mediated by the action of insulin on insulin receptors. This has indeed been found to be the case in several tissues of hibernating ground squirrels where 
PI3K mediated activation of Akt was suppressed during torpor (Cai et al., 2004; Abnous et al., 2008). A lack of PI3K/Akt signaling affects downstream events that cause an eventual decrease in lipogenesis and glycogen synthesis (Porstmann et al., 2008; Chakrabarti et al., 2010; Duvel et al., 2010; Laplante and Sabitini, 2010; Li et al., 2010). Furthermore, a drop in circulating insulin leads to decreased glucose uptake into cells and concomitant decrease in glycolytic output. This decrease, however, appears to be augmented in several tissues by the coordinated control of several important glycolytic enzymes. For instance, within the mammalian liver, glycogen phosphorylase (GP), phosphofructokinase (PFK), pyruvate kinase (PK), and pyruvate dehydrogenase (PDH) activity have all been found to be suppressed through the mechanism of reversible phosphorylation during mammalian torpor (Storey, 1987, Storey, 1989; Brooks and Storey, 1992; Heldmaier et al., 1999). Similarly, within mammalian skeletal muscle, hexokinase (HK) was found to be regulated by reversible phosphorylation - the highly phosphorylated and less active form of HK being present in the torpid animal (Abnous and Storey, 2008). Additionally, and in contrast to responses by mammalian liver, studies on skeletal muscle GP, PFK, PK, and PDH showed no kinetic difference between euthermic and hibernating animals (Brooks and Storey, 1992; Heldmaier et al., 1999). Few studies have been conducted in other tissues but the aforementioned investigations do indicate some level of tissue-specific regulation of glycolytic output.

The torpor-induced alterations to the gluconeogenic pathway are somewhat less clear than those elucidated for glucose and lipid catabolism. Insulin levels present during torpor suggest that liver and muscle glycogen synthesis would be suppressed, and some studies have shown this to be the case. For instance, Burlington and Klain (1967) 
indicated a net decrease in liver glycogen synthesis during torpor, while several independent studies have suggested similar results in hibernator skeletal muscle (Galster and Morrison, 1970; Galster and Morrison, 1975). One study has even shown that gluconeogenic rates declined within torpid hepatocytes as compared to the control hepatocytes (Gehnrich and Aprille, 1988). In contrast, several studies have indicated that gluconeogenesis is maintained or slightly increased for hepatic (Burlington and Klain, 1967; Klain and Whitten, 1968; Green et al., 1984; Staples and Hochachka, 1998) and skeletal muscle (Hannon and Vaughn, 1961; el Hachimi et al., 1990; James et al., 2013) tissue during torpor.

Interbout Arousal

The periods of arousal that divide extended torpor bouts are critical for survival over the hibernating season, and are periods of high energy expenditure. For instance, energy usage during re-warming and the short periods of arousal consumes $40-70 \%$ of the energy budget for the entire hibernation cycle (Wang, 1979). This large fraction of total energy stored gets utilized mainly in the re-warming process. Re-warming begins within the brown adipose tissue with non-shivering thermogenesis (NST). NST makes use of uncoupling protein 1 (UCP1) within the inner mitochondrial membrane to create a bypass for the proton gradient generated by the electron transport chain, thereby allowing the energy that would have been used to synthesize ATP to be dissipated as heat. Once the animal has warmed to a sufficient degree (around $15^{\circ} \mathrm{C}$ ), shivering thermogenesis can commence and euthermia is eventually reached. The fuel usage during the initial rewarming is thought to originate mainly from lipid oxidation, with carbohydrates being used increasingly as $T_{b}$ increases. Indeed, RQ values of $\sim 0.7$ are commonly seen during 
the initial re-warming indicating that fatty acids constitute the main fuel used for NST. The RQ value then jumps significantly to values $>0.85$ during interbout euthermia, indicating that either protein or carbohydrates contribute to energy expenditure during the short period of time that the mammal spends at normal $\mathrm{T}_{\mathrm{b}}$ (Buck and Barnes, 2000; Karpovich et al., 2009). Protein catabolism is unlikely in this situation since plasma urea and non-protein nitrogen levels do not indicate the breakdown of proteins for energy during interbout arousal (Galster and Morrison, 1975; Zimmerman, 1982). This is substantiated by the fact that skeletal muscle glycogen reserves appear to decrease as the mammal proceeds from early to late arousal indicating the use of carbohydrates during this period (Galster and Morrison, 1975).

\section{Hypothesis}

Given the apparent changes in flux in carbohydrate metabolism between the various stages of hibernation (i.e. pre-hibernation euthermia, torpor, and interbout arousals), I propose that glucose catabolism and anabolism (pathways illustrated in Appendix IF) are regulated at the enzyme level within ground squirrel skeletal muscle to facilitate rapid alterations in enzyme state without necessitating energetically costly changes in protein expression. Two main hypotheses guide the research in this thesis:

(1) In conjunction with the suppression of skeletal muscle hexokinase, other crucial glycolytic enzymes are regulated and suppressed by posttranslational modifications to aid in coordinating skeletal muscle carbohydrate catabolism during torpor.

(2) Gluconeogenesis is suppressed during torpor, coordinated and mediated by the posttranslational modification and regulation of key gluconeogenic enzymes. 


\section{Objectives}

The extended torpor bouts that occur during hibernation are highly regulated physiological states that not only involve a significant depression of metabolic rates but also a major reorganization of metabolism to support long term survival at low temperatures. One aspect of metabolism that has been understudied to date is the regulation of skeletal muscle glycolysis and gluconeogenesis pathways during mammalian torpor (Appendix IF). The research in this thesis investigates the physical, kinetic and regulatory properties of an important glycolytic enzyme, pyruvate kinase (PK; Chapter 3 a bidirectional enzyme that is crucial to both glycolytic and gluconeogenic pathways, glyceraldehyde-3-phosphate dehydrogenase (GAPDH; Chapter 2), and an enzyme that functions solely in a gluconeogenic capacity, fructose-1,6-bisphosphatase (FBPase; Chapter 4). Analysis of these important enzymes of carbohydrate metabolism provide a more clear understanding of the state of glucose anabolism versus catabolism during ground squirrel torpor, as well as provide insight into the regulation of these pathways.

In addition to the above mentioned glycolytic/gluconeogenic enzymes, two potentially important regulatory enzymes that impact carbohydrate metabolism are also analyzed in this thesis: protein phosphatase 2A (Chapter 5) and glycogen synthase kinase-3 (Chapter 6). Specifically, the activity states and control mechanisms (if any) of these two signal transduction enzymes are examined to determine their potential role during ground squirrel torpor. Overall, then, the studies in this thesis are aimed at clarifying the key enzyme players, their activity status and regulatory mechanisms in skeletal muscle carbohydrate metabolism during mammalian hibernation. 


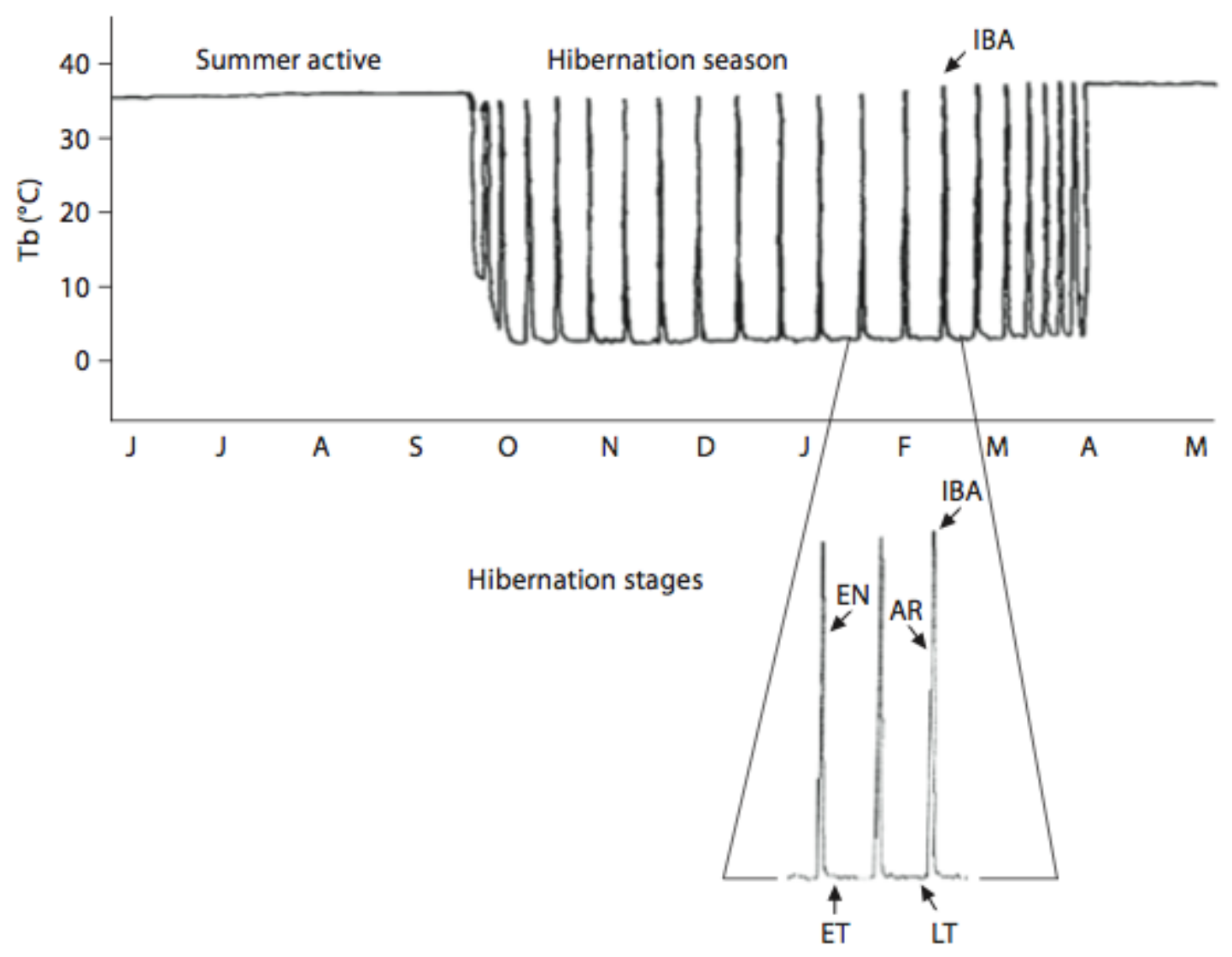

Figure 1.1. The body temperature of a golden-mantled ground squirrel

(Callospermophilus lateralis) monitored over one year. The inset figure depicts three torpor-arousal cycles with indicated stages of: entrance into torpor (EN), early torpor (ET), late torpor (LT), arousal from torpor (AR) and interbout arousal (IBA). The figure was taken from Carey et al., (2003). 


\section{Chapter 2}

\section{Purification and Properties of Glyceraldehyde-3- phosphate Dehydrogenase from the Skeletal Muscle of the Hibernating Ground Squirrel, Ictidomys tridecemlineatus}




\section{Introduction}

Glyceraldehyde-3-phosphate dehydrogenase (GAPDH; EC 1.2.1.12) is an integral member of many cellular events within the cell. This enzyme has recently been shown to be involved in such diverse functions as membrane trafficking, oxidative stress response, apoptosis, autophagy and the regulation of gene expression (reviewed in Sirover, 2011; Tristan et al., 2011). This, of course, is in addition to its canonical role in the glycolytic and gluconeogenic pathways where it converts D-glyceraldehyde-3-phosphate and NAD ${ }^{+}$ to 1,3-bisphosphoglycerate and NADH, or vice versa (structures illustrated in Appendix IG). The diversity of GAPDH roles in a variety of pathways makes it a potentially important enzyme to investigate within an animal that experiences extreme environmental stress.

One of the most dramatic adaptations to environmental stress is mammalian hibernation, which is adopted by a select number of mammals in the face of subzero temperatures and dangerously low food availability during prolonged winters. The bouts of aerobic torpor that occur during hibernation require many physiological and biochemical changes aimed at suppressing energetically expensive processes and reestablishing a new balance between ATP consumption and production. Of particular importance in this regard are the changes observed in skeletal muscle glucose metabolism during torpor. During dormancy, skeletal muscle carbohydrate catabolism is thought to be suppressed, with lipid catabolism becoming the primary source for energy (cited in Buck and Barnes, 2000; Tashima et al., 1970). The state of gluconeogenesis on the other hand is somewhat less clear during dormancy. Several studies indicate that there could be an increase in gluconeogenesis (Hannon and Vaughn, 1961; el Hachimi et al., 1990; 
James et al., 2013), while others indicate a decrease in gluconeogenesis within the skeletal muscle during torpor (Zimny and Tyrone, 1957; Galster and Morrison, 1970; Galster and Morrison, 1975). I hypothesized that skeletal muscle GAPDH activity, in both gluconeogenic and glycolytic directions, becomes suppressed during torpor, and that this suppression is mediated by reversible posttranslational modification. The present chapter explores GAPDH properties and regulation in ground squirrel skeletal muscle in an attempt to clarify the state of carbohydrate metabolism during torpor.

\section{Materials and Methods}

Animals

Thirteen-lined ground squirrels (Ictidomys tridecemlineatus) were captured in the fall in Illinois and transported to an animal care facility on the National Institutes of Health campus in Bethesda, Maryland. Hibernation experiments were approved by the Animal Care and Use Committee of the National Institute of Neurological Disorders and Stroke and conducted there as previously reported (Mamady and Storey, 2006). Individuals denoted as hibernators were sampled between December and February and only when animals had been in continuous torpor for at least 3 days, as evidenced by continuous low body temperature $\left(\sim 6^{\circ} \mathrm{C}\right)$ monitored by a subepidermal sensor (denoted as long hibernation or LH). Animals that had not entered torpor after at least 3 days in the cold room and showed a continuous high body temperature $\left(36-38^{\circ} \mathrm{C}\right)$ were sampled as euthermic controls (denoted as active in the cold room or ACR). All animals were sacrificed by decapitation and tissues were quickly excised and frozen in liquid nitrogen. Tissues were then transported by air to Carleton University on dry ice where they were subsequently stored at $-80^{\circ} \mathrm{C}$. 


\section{Purification of GAPDH}

Frozen skeletal muscle samples were homogenized 1:5 w:v in buffer A $(25 \mathrm{mM}$ imidazole, $\mathrm{pH}$ 7.0, $1.25 \mathrm{mM}$ EDTA, $1.25 \mathrm{mM}$ EGTA, $12.5 \mathrm{mM} \beta$-glycerophosphate, 5 $\mathrm{mM} \beta$-mercaptoethanol, 5\% v:v glycerol, and a few crystals of phenylmethylsulphonyl fluoride added just prior to homogenization) using a Polytron homogenizer. Homogenates were centrifuged at $13,500 \mathrm{~g}$ for 30 minutes at $5^{\circ} \mathrm{C}$, after which supernatants were decanted and held on ice until use in the first purification step.

A blue-agarose column was prepared with the dimensions of $1 \times 3.5 \mathrm{~cm}$ (diameter $\mathrm{x}$ height) and equilibrated in $15 \mathrm{~mL}$ of buffer $\mathrm{A}$. A $1.5 \mathrm{~mL}$ aliquot of crude muscle extract was then loaded on the column, which was then washed with $30 \mathrm{~mL}$ of buffer A. This was sufficient to reduce the absorbance of the fractions at $280 \mathrm{~nm}$ to that of the buffer alone. GAPDH was then eluted with a $0-1 \mathrm{M} \mathrm{KCl}$ gradient in buffer $\mathrm{A}$ and activity was assayed under $\mathrm{V}_{\max }$ conditions. The top three activity fractions were pooled and applied to a phenyl-agarose column.

The phenyl-agarose column was prepared with the same dimensions as for the blue-agarose column, and equilibrated in $15 \mathrm{~mL}$ buffer B (buffer A containing $1 \mathrm{M} \mathrm{KCl}$ ). Salt was also added to the pooled enzyme from the blue-agarose column to bring the concentration to $1 \mathrm{M} \mathrm{KCl}$ and then the enzyme was applied to the phenyl-agarose column. The column was washed with $30 \mathrm{~mL}$ of buffer $\mathrm{B}$, which was sufficient to wash away any unbound protein. GAPDH was eluted with a linear 1-0 $\mathrm{M} \mathrm{KCl}$ gradient (i.e. declining salt concentration) and assayed under $\mathrm{V}_{\max }$ conditions. The three top activity fractions were pooled and held at $4{ }^{\circ} \mathrm{C}$ until use.

GAPDH Assay 
GAPDH was assayed spectrophotometrically at $340 \mathrm{~nm}$ using a Thermo Labsystems Multiskan spectrophotometer. Optimal assay conditions for the oxidation of D-glyceraldehyde-3-phosphate (GAP) were 2.5 mM GAP, 1.5 mM NAD ${ }^{+}, 15 \mathrm{mM}$ $\mathrm{Na}_{2} \mathrm{HAsO}_{4}$, and $50 \mathrm{mM} \mathrm{NaH}_{2} \mathrm{PO}_{4}, \mathrm{pH}$ 8.0. Optimal assay conditions for the reverse reaction were 2.5 mM 3-phosphoglycerate (3PG), 2 mM Mg-ATP, 0.1 mM NADH, 1 Unit of phosphoglycerate kinase, and $50 \mathrm{mM}$ Tris- $\mathrm{HCl}$ buffer, $\mathrm{pH}$ 8.0. The coupling enzyme was added in excess to ensure the measurement of GAPDH activity and not phosphoglycerate kinase activity. Excessive activity was ensured by doubling the amount of enzyme in the reaction and checking to make sure the reaction rate doubled. This was done at all temperatures studied. Regardless of the assay direction, the enzyme reaction was initiated by the addition of $10 \mu \mathrm{L}$ of purified GAPDH. The amount of enzyme was optimized to ensure the measurement of initial reaction rates. Purified enzyme samples did not lose significant amounts of enzyme activity for up to two weeks (data not shown). All experiments were conducted within this time period.

Microplate analysis (MPA) and Kinetics v.3.51 programs (Brooks, 1992; 1994) were used to analyze the data and determine Michaelis constants $\left(\mathrm{K}_{\mathrm{m}}\right)$ for substrates and inhibitor concentrations that reduced enzyme activity by $50 \%\left(\mathrm{I}_{50}\right)$ values. All points were fitted to a curve determined by the Hill equation $(\mathrm{h}>0)$ within the Kinetics program. Soluble protein was measured using the Coomassie Brilliant Blue dye binding method with the Bio-Rad prepared reagent and a standard curve created using bovine serum albumin.

Effect of Temperature on GAPDH Activity 
To assess the effect of temperature on GAPDH activity, various kinetic parameters were determined at low $\left(5 \pm 1^{\circ} \mathrm{C}\right)$ and high $\left(36 \pm 1^{\circ} \mathrm{C}\right)$ temperature. To establish the low temperature condition, the Thermo Labsystems Multiskan spectrophotometer and the microplates containing all solutions except for purified GAPDH (being cooled separately) were placed inside a temperature-controlled incubator set to $5^{\circ} \mathrm{C}$. To establish the high temperature conditions, the microplate chamber of the Thermo Labsystems Multiskan spectrophotometer was set to warm to $37^{\circ} \mathrm{C}$ and the microplates (containing all solutions except for the purified GAPDH) were warmed on a EchoThermomicroplate heater (Torrey Pines Scientific). It is important to note that for assays at $36^{\circ} \mathrm{C}$ the enzyme was not allowed to equilibrate at this high temperature due to the lack of stability of GAPDH when heated (experimentally identified in this study). Prior to assay, the well temperature was monitored by a digital telethermometer placed inside the well. The final temperature of the well was also determined after the assay to ensure a stable reaction temperature.

The thermal stability of GAPDH was assessed in a manner similar to that described by Iddar et al. (2003). Briefly, aliquots of purified GAPDH were incubated in a water bath at a given temperature for 15 minutes, after which they were immediately chilled on ice and assayed under optimal conditions at $25^{\circ} \mathrm{C}$.

The effects of temperature on GAPDH were also analyzed using Arrhenius plots to determine GAPDH activation energy $\left(\mathrm{E}_{\mathrm{a}}\right)$. The maximal velocity $\left(\mathrm{V}_{\max }\right)$ of GAPDH in the $1,3 \mathrm{BPG}$-reducing reaction were measured at $5^{\circ} \mathrm{C}$ intervals from $5-35^{\circ} \mathrm{C}$. Assays at temperatures below or above $25^{\circ} \mathrm{C}$ (room temperature) were conducted as described 
above for the $5^{\circ} \mathrm{C}$ and $36^{\circ} \mathrm{C}$ assays, respectively. Well temperature was again confirmed using a telethermometer at the end of each assay.

Effect of Protein Oxidation on GAPDH Activity

The oxidizing agent, 2,2-azobis(2-amidinopropane dihydrochloride) (AAPH) was used to determine the susceptibility of GAPDH to inactivation through protein oxidation. $\mathrm{I}_{50}$ values for AAPH were determined by incubating purified GAPDH extracts with various concentrations of AAPH $(0-150 \mathrm{mM})$ for $20 \mathrm{~h}$ at $4^{\circ} \mathrm{C}$, and subsequently assaying for $\mathrm{V}_{\max }$ in the GAP-oxidizing direction at room temperature.

\section{Differential Scanning Fluorimetry}

Differential scanning fluorimetry (DSF) is a high throughput method that monitors the thermal unfolding of proteins in the presence of a fluorescent dye (Niesen et al., 2007). Purified ACR and LH GAPDH were spun through a G50 Sephadex column, pre-equilibrated in DSF buffer (100 mM potassium phosphate, $\mathrm{pH} 7.0,150 \mathrm{mM} \mathrm{NaCl})$, at $2500 \mathrm{rpm}$ for 1 minute. Protein samples were then aliquoted to a concentration of 0.05 $\mu \mathrm{g} / \mu \mathrm{L} /$ well into the wells of a 48 -well, thin-walled PCR plate along with the dye SYPRO Orange (5X final concentration of the dye). PCR plates were then sealed with sealing tape and placed into a BioRad iCycler5 PCR instrument. SYPRO Orange (Invitrogen) fluorescence was monitored as described by Biggar et al., 2012). Briefly, SYPRO Orange was excited through the transmission of light through the FAM filter $(485 \pm 30$ $\mathrm{nm})$, with the subsequent emission of light through the ROX filter $(625 \pm 30 \mathrm{~nm})$. Measurements were taken every $30 \mathrm{~s}$ in $1^{\circ} \mathrm{C}$ increments from $25^{\circ} \mathrm{C}$ to $97^{\circ} \mathrm{C}$. Subsequent analysis of the fluorescent data using OriginPro 8.5 and the Boltzmann distribution curve 
yielded the midpoint temperature of the protein-unfolding transition, known as the protein melting temperature $\left(\mathrm{T}_{\mathrm{m}}\right)$, for GAPDH.

\section{GAPDH Digestion and Mass Spectrometry}

Purified GAPDH samples were digested in $50 \mathrm{mM}$ ammonium bicarbonate containing sequencing grade porcine trypsin at $0.013 \mu \mathrm{g} / \mu \mathrm{L}$ (Promega). Extracted peptides were dried down in a vacuum apparatus overnight followed by resuspension in $30 \mu \mathrm{L}$ of $0.1 \%$ formic acid for use in subsequent mass spectrometry. Mass analysis was carried out on an ESI-TOF QUAD-MS/MS. Samples were loaded onto a $5 \mu \mathrm{m} \times 50 \mathrm{~mm}$ column packed in-house with $5 \mu \mathrm{m} \mathrm{C18}$ beads (100 ^̊ pore size) (Michrom Bioresources, CA) at a rate of $300 \mu \mathrm{L} /$ min using a Dinoex 3000 ultimate HPLC system (Thermo Fisher Scientific, IL). Peptides were eluted using a $0-60 \%(\mathrm{v} / \mathrm{v})$ gradient of acetonitrile with $0.1 \%$ formic acid over $30 \mathrm{~min}$. Mass spectra were analyzed using Mascot (Matrix Science).

SDS Polyacrylamide Gel Electrophoresis, Immunoblotting, and ProQ Diamond Phosphoprotein Staining

Purified ACR and LH GAPDH samples were mixed 2:1 (v:v) with SDS loading buffer (100 mM Tris buffer, pH 6.8, 4\% w:v SDS, $20 \%$ v:v glycerol, $0.2 \%$ w:v bromophenol blue, and 10\% v:v 2-mercaptoethanol) and boiled for 5 minutes, cooled on ice and frozen at $-20^{\circ} \mathrm{C}$ until use.

SDS resolving gels (15\% v/v acrylamide, $400 \mathrm{mM}$ Tris, $\mathrm{pH} 8.8,0.1 \% \mathrm{w} / \mathrm{v}$ SDS, $0.2 \% \mathrm{w} / \mathrm{v}$ ammonium persulfate [APS], $0.04 \% \mathrm{v} / \mathrm{v}$ TEMED) were prepared with a $5 \%$ stacking gel (5\% acrylamide, $190 \mathrm{mM}$ Tris, $\mathrm{pH} 6.8,0.1 \% \mathrm{w} / \mathrm{v}$ SDS, $0.15 \% \mathrm{w} / \mathrm{v}$ APS, $0.1 \% \mathrm{v} / \mathrm{v}$ TEMED). Purified ACR and LH GAPDH were loaded onto these gels and 
separated electrophoretically in SDS-PAGE running buffer (25 mM Tris-base, $\mathrm{pH} 8.5$, $190 \mathrm{mM}$ glycine, and $0.1 \% \mathrm{w} / \mathrm{v} \mathrm{SDS}$ ) at $180 \mathrm{~V}$ for $45 \mathrm{~min}$. A $3 \mu \mathrm{L}$ aliquot of Spectra ${ }^{\mathrm{TM}}$ Multicolor Broad Range Protein Ladder was added to one lane of every gel to provide molecular weight markers. Commercially purified rabbit muscle GAPDH (Boehringer Mannheim) was also loaded onto the gel to confirm the correct location of GAPDH subunits. Following electrophoresis, proteins were either electroblotted onto polyvinylidiene difluoride (PVDF) membranes (Millipore) by wet transfer and used for immunoblotting or fixed and stained with ProQ diamond phosphoprotein stain.

With regards to phosphoprotein staining, the SDS-gel containing purified ACR and LH GAPDH was washed three times in fixing solution $(50 \% \mathrm{v}: \mathrm{v}$ methanol, $10 \% \mathrm{v}: \mathrm{v}$ acetic acid) and subsequently left overnight at $4^{\circ} \mathrm{C}$ in this solution. The following day the gel was washed three times for ten minutes each time in $\mathrm{ddH}_{2} \mathrm{O}$, after which the gel was immersed in ProQ Diamond phosphoprotein stain (Invitrogen, Eugene, OR) for 30 minutes. Once the stain was placed onto the gel, the gel remained covered in aluminum foil to protect the light-sensitive stain. After staining, the gel was washed three times for 10 minutes each time with $\mathrm{ddH}_{2} \mathrm{O}$ before being viewed in a ChemiGenius Bioimaging System (Syngene, Fredrick, MD). The fluorescent bands were then quantified using GeneTools software. Subsequently, the SDS-gel was stained with Coomassie blue (0.25\% w:v Coomassie Brilliant Blue $\mathrm{R}$ in 50\% v/v methanol, $7.5 \% \mathrm{v}: \mathrm{v}$ acetic acid). The GAPDH ProQ band intensities were subsequently standardized against the Coomassie blue-stained GAPDH band intensities to negate errors caused by sample loading. Immunoblottting began with the wet transfer of proteins from $15 \%$ SDS gels (run as above) onto PVDF membranes previously soaked in methanol. Electroblotting was 
performed at room temperature for $1.5 \mathrm{~h}$ at $160 \mathrm{~mA}$ under transfer buffer $(25 \mathrm{mM}$ Tris, pH 8.5, $192 \mathrm{mM}$ glycine, and 20\% v/v methanol). Following protein transfer, PVDF membranes were blocked with 2.5\% skim milk for 20 minutes. Blots were then washed three times for 5 minutes each and then incubated overnight at $4{ }^{\circ} \mathrm{C}$ with one of the following primary antibodies that detect specific posttranslational modifications to the enzyme: (1) Pan-acetyl (Santa Cruz Biotechnology), (2) Phospho-serine (Calbiochem), (3) Phospho-threonine (Invitrogen), (4) Phospho-tyrosine (Invitrogen), or (5) Methyllysine (StressMarq Biosciences Inc.). All primary antibodies were diluted 1:1000 v:v in Tris-buffered saline with Tween-20 (TBST; $20 \mathrm{mM}$ Tris-base, $140 \mathrm{mM} \mathrm{NaCl,} \mathrm{0.05 \%}$ Tween-20) with a small amount of sodium azide added. After the overnight incubation, membranes were washed with TBST three times for 5 minutes each, followed by incubation with the appropriate secondary antibody conjugated with horseradish peroxidase (Bioshop Canada) at a dilution of 1:4000 v:v in TBST. Membranes were incubated at room temperature for $1.5 \mathrm{~h}$, and then washed three times for 5 minutes each time with $\mathrm{ddH}_{2} \mathrm{O}$. Signal was then detected using enhanced chemiluminescence (ECL), initiated by the addition of $600 \mu \mathrm{L}$ of hydrogen peroxide and $600 \mu \mathrm{L}$ of luminol reagent to the membrane's surface for several seconds. The mixture was then poured off and the chemiluminescence was detected with the ChemiGenius Bioimaging System (Syngene, MD, USA). Membranes were then stained with Coomassie blue $(0.25 \% \mathrm{w} / \mathrm{v}$ Coomassie Brilliant Blue $\mathrm{R}$ in 50\% methanol, 7.5\% acetic acid) and destained with destaining solution $\left(60 \% \mathrm{v} / \mathrm{v}\right.$ methanol, $20 \% \mathrm{v} / \mathrm{v}$ acetic acid in $\left.\mathrm{ddH}_{2} \mathrm{O}\right)$ until bands were clearly seen. Band densities were analyzed using the associated GeneTools software, and were normalized against the corresponding Coomassie blue stained bands. 
In vitro Incubations that Stimulate Protein Kinases or Protein Phosphatases

Crude muscle extracts were prepared as described above, and then subsequently filtered through a small G50 Sephadex column that had been pre-equilibrated in buffer B (25 mM imidazole, 5\% v:v glycerol, $5 \mathrm{mM} 2$-mercaptoethanol, $\mathrm{pH}$ 7.0). Aliquots of the filtered supernatants were incubated overnight at $4{ }^{\circ} \mathrm{C}$ with specific inhibitors and stimulators of protein kinases or protein phosphatases, as described in MacDonald and Storey (1999). Each aliquot was mixed 1:2 v:v with buffer B plus the following additions:

(1) Stop conditions: $1.25 \mathrm{mM}$ EGTA, $1.25 \mathrm{mM}$ EDTA, and $12.5 \mathrm{mM}$ ßglycerophosphate.

(2) Protein Tyrosine Phosphatases (PTP) - $30 \mathrm{mM} \mathrm{NaF}$

(3) Alkaline Phosphatase (Alk PPase) - $10 \mathrm{mM} \mathrm{MgCl} 2+5 \mathrm{mM}$ EDTA $+1 \mathrm{U}$ calf intestine alkaline phosphatase per incubation tube.

(4) Protein Kinase $\mathrm{C}(\mathrm{PKC})-1.3 \mathrm{mM} \mathrm{CaCl} 2+7 \mu \mathrm{g} / \mathrm{mL}$ phorbol 12-myristate 13-acetate $(\mathrm{PMA})+5 \mathrm{mM} \mathrm{ATP}+5 \mathrm{mM} \mathrm{MgCl}_{2}+30 \mathrm{mM} \mathrm{NaF}+5 \mathrm{mM} \mathrm{Na}_{3} \mathrm{VO}_{4}$

(5) AMP-activated Protein Kinase (AMPK) - $1 \mathrm{mM} \mathrm{AMP}+5 \mathrm{mM} \mathrm{ATP}+10 \mathrm{mM} \mathrm{MgCl} 2$ $+30 \mathrm{mM} \mathrm{NaF}+5 \mathrm{mM} \mathrm{Na}_{3} \mathrm{VO}_{4}$.

(6) Calcium/calmodulin Activated Protein Kinase (CaMK) - $1 \mathrm{U}$ of calmodulin/incubation tube $+1.3 \mathrm{mM} \mathrm{CaCl}_{2}+5 \mathrm{mM} \mathrm{ATP}+10 \mathrm{mM} \mathrm{MgCl}_{2}+30 \mathrm{mM}$ $\mathrm{NaF}+5 \mathrm{mM} \mathrm{Na}_{3} \mathrm{VO}_{4}$

Samples were spun at 2500 RPM through a G50 Sephadex column that had been pre-equilibrated in buffer A. This removed small molecular weight molecules and 
allowed GAPDH activity to be reassessed in the GAP-oxidizing direction under optimal conditions.

Preparation of Cytoplasmic and Nuclear Fractions

Thirteen-lined ground squirrel skeletal muscle cytoplasmic and nuclear extracts were prepared using the method described previously (Mamady and Storey, 2006). Briefly, $0.5 \mathrm{~g}$ of skeletal muscle tissue was gently homogenized in buffer C $(10 \mathrm{mM}$ HEPES, $\mathrm{pH}$ 7.9, $10 \mathrm{mM} \mathrm{KCl}, 10 \mathrm{mM}$ EDTA, $10 \mathrm{mM}$ DTT and $1.5 \mu \mathrm{L}$ protease inhibitor cocktail (Sigma)) using a Dounce homogenizer at $4^{\circ} \mathrm{C}$. Muscle cell nuclei were pelleted by centrifugation at $8000 \mathrm{xg}$ for $10 \mathrm{~min}$. The resulting supernatant was removed (this is the cytoplasmic fraction) and the pellet was washed in buffer C. Following washing, the pellet was resuspended in extraction buffer (20 mM HEPES, pH 7.9, $400 \mathrm{mM} \mathrm{NaCl}, 1$ $\mathrm{mM}$ EDTA, $10 \% \mathrm{v} / \mathrm{v}$ glycerol, $10 \mathrm{mM}$ DTT, and $1.5 \mu \mathrm{L}$ protease inhibitor cocktail) and placed on a rocker at $4^{\circ} \mathrm{C}$ for $1 \mathrm{~h}$. The resuspended sample was then centrifuged at 10 $000 \mathrm{x} g$ for $10 \mathrm{~min}$, whereby the resulting supernatant (this is the nuclear fraction) was collected. Protein concentration was determined as described above and samples of 5-10 $\mu \mathrm{g}$ (final amount) were made in SDS loading buffer (recipe defined above). Proteins were electrophoretically separated and immunoblotted as described above. Blots were probed with GAPDH primary antibody (1:1000; Santa Cruz Biotechnology) in the same manner as previously described.

\section{GAPDH Bioinformatics}

The nucleotide and amino acid sequence of 13-lined ground squirrel GAPDH was deduced using multiple GAPDH sequences from a variety of animals. As the basis for the construction, the rat (Rattus norgevicus) GAPDH coding nucleotide sequence (NCBI 
Reference Sequence: NM_017008.3) was blasted against the whole genome shotgun sequences from the 13-lined ground squirrel genome, and the sequence with the highest coverage was taken (Accession Number: gb|AAQQ01785922.1|). This sequence was then compared to three other whole genome shotgun sequences of 13-lined ground squirrel GAPDH as well as a low-coverage 1.9X 13-lined ground squirrel GAPDH sequence produced by the Broad Institute (http://www.ensembl.org/Spermophilus_tridecemlineatus/Info/Index). When discrepancies existed between the whole genome shotgun reads and the Ensemble sequence, typically, the whole genome shotgun sequence was taken as true. However, if there were discrepancies between whole genome shotgun reads themselves as well as the ensemble sequence, then GAPDH sequences from the rat, mouse, pig and cow were used to help decipher the most likely nucleotide sequence. Sequences were compared using DNAMAN Version 4.0 (Lynnon BioSoft).

\section{Results}

\section{Purification of GAPDH}

GAPDH from skeletal muscle of 13-lined ground was purified using a Blueagarose affinity column followed by a hydrophobic column, phenyl-agarose. The overall yield of the final ACR GAPDH preparation was $45 \%$ with a fold purification of 460 and a final specific activity of $146 \mathrm{U} / \mathrm{mg}$ (Table 2.1). The overall yield was similar for LH GAPDH preparations (data not shown). This purification procedure yielded electrophoretically pure preparations that were used in all subsequent experiments (Fig. 2.1).

GAPDH Kinetics 
Kinetic parameters of purified ACR and LH GAPDH were assessed in both the GAP-oxidizing direction and the 1,3-bisphosphoglycerate (1,3BPG)-reducing direction. It is important to note that commercial 3-phosphoglycerate kinase was used to rapidly convert 3-phosphoglycerate into 1,3BPG for use in the GAPDH reaction, thus kinetic parameters for 1,3BPG were measured indirectly in this study (although phosphoglycerate kinase activity was always in high excess in the assay).

The activity of GAPDH in both directions was investigated at two temperatures, one that corresponded to approximately euthermia $\left(36^{\circ} \mathrm{C}\right)$ and one typical of torpor $\left(5^{\circ} \mathrm{C}\right)$. Several significant differences in GAPDH kinetics were observed between ACR and LH GAPDH under high and low temperatures. At $5^{\circ} \mathrm{C}$, the $\mathrm{V}_{\max }$ values in the GAP-oxidizing direction and 1,3BPG-reducing directions were $\sim 63 \%$ and $44 \%$ less, respectively, for $\mathrm{LH}$ GAPDH as compared to ACR GAPDH (Table 2.2). At the same temperature, the $\mathrm{K}_{\mathrm{m}}$ $\mathrm{NAD}^{+}$for LH GAPDH was $60 \%$ lower than that of ACR GAPDH. At $36^{\circ} \mathrm{C}, \mathrm{GAPDH}$ $\mathrm{V}_{\max }$ in the GAP-oxidizing direction was significantly different between ACR and LH purified samples, LH GAPDH $V_{\max }$ being $\sim 67 \%$ less as compared to ACR GAPDH. Similar to the situation at $5^{\circ} \mathrm{C}$, the $\mathrm{K}_{\mathrm{m}} \mathrm{NAD}^{+}$was $60 \%$ lower for $\mathrm{LH}$ GAPDH as compared to the euthermic value (Table 2.2). $\mathrm{K}_{\mathrm{m}} \mathrm{GAP}$ and $\mathrm{K}_{\mathrm{m}}$ 3PG did not differ between the ACR and LH enzyme at either temperature.

The maximal activity of GAPDH was also monitored over a range of temperatures between $5^{\circ} \mathrm{C}$ and $36^{\circ} \mathrm{C}$ to determine the activation energy $\left(\mathrm{E}_{\mathrm{a}}\right)$ for the 1,3BPG-reducing reaction of GAPDH. The calculated $\mathrm{E}_{\mathrm{a}}$ for $\mathrm{LH}$ GAPDH was 2-fold higher than the corresponding value for ACR GAPDH (Table 2.3). GAPDH Stability and Effectors 
To assess the thermal stability of each enzyme form, the purified extracts were subjected to short (15 min) incubations at different temperatures, after which GAPDH was assayed at room temperature. Figure 2.2 indicates that LH GAPDH appears to be significantly less susceptible to thermal denaturation as compared to ACR GAPDH, with 45 or $55^{\circ} \mathrm{C}$ temperatures being required to cause a significant decrease in enzyme activity as compared to 35 and $45^{\circ} \mathrm{C}$ for ACR GAPDH. Furthermore, LH GAPDH displayed a significantly higher thermal melting point of $56.1 \pm 0.2$ as compared to the euthermic GAPDH $\mathrm{T}_{\mathrm{m}}$ of $54.7 \pm 0.1$ (Table 2.3$)$.

GAPDH susceptibility to oxidation was assessed through exposure of purified enzyme to increasing concentrations of AAPH. There was no significant difference in the concentration of AAPH that was required to cause $50 \%$ inactivation of ACR and LH GAPDH (Table 2.3).

\section{GAPDH Posttranslational Modification}

Posttranslational modifications of purified ACR and LH GAPDH were analyzed first by ESI-QUAD TOF MS/MS. Peptides produced from a tryptic digestion of GAPDH were compared to known sequences of rat, mouse, and human GAPDH amino acid sequences facilitated by the Mascot program. Analysis of the trypsin digested ACR GAPDH resulted in 33\% sequence coverage as compared to the deduced protein sequence of 13-lined ground squirrel GAPDH (Appendix IA). Within the peptides detected, numerous posttranslational modifications were identified including dimethylation, acetylation, oxidation, and phosphorylation (Table 2.4). Trypsin digested LH GAPDH produced peptides that provided $38 \%$ sequence coverage when compared to the deduced 13-lined ground squirrel sequence (Appendix IB). Similar to ACR GAPDH, 
LH GAPDH peptides contained a variety of modifications including acetylation, methylation, and dimethylation. Comparing the peptides sequences detected in both ACR and LH GAPDH preparations, it is clear that several similar sequences were found. Indeed, $\sim 82 \%$ of the ACR GAPDH sequence covered by the trypsin-digested peptides was also observed in sequences recovered from LH GAPDH mass spectrometry. Specific differences were found between GAPDH from ACR and LH conditions with respect to posttranslational modifications identified, details of which will be explored in the Discussion.

Subsequently, analysis of purified GAPDH by immunoblotting and phosphoprotein staining aimed at confirming the different levels of modified residues between the control and hibernating conditions. The overall phosphorylation state of GAPDH was assessed by ProQ Diamond phosphoprotein staining of the purified enzyme on an SDS-gel. Figure 2.3 shows that there was no significant difference between ACR and LH GAPDH relative band intensities for total protein-bound phosphate. Similarly, Western blotting with antibodies detecting phospho-serine or phospho-threonine indicated no significant difference in these posttranslational modifications between ACR and LH GAPDH (Fig. 2.3). However, GAPDH from the two conditions differed significantly in phospho-tyrosine content with LH GAPDH showing 35\% less phosphorylation as compared to the euthermic enzyme (Fig. 2.3).

Western blots looking at the overall acetylation level of purified GAPDH showed no significant difference between control and torpid enzyme forms (Fig. 2.4). The same results were seen when probing purified GAPDH with anti-methyl-lysine antibody, no significant differences between ACR and LH conditions. 


\section{GAPDH Regulation by Reversible Phosphorylation}

Crude skeletal muscle samples were incubated in solutions that either stimulated the activities of selected endogenous protein kinases or phosphatases or in a solution that activated commercial alkaline phosphatase and then GAPDH activity was reassessed at

room temperature. Incubations that aimed to stimulate endogenous tyrosine phosphatases (PTP) did not have any significant effect on GAPDH activity while the action of commercial alkaline phosphatase (Alk PPase) caused a 50\% increase in ACR GAPDH activity when compared to ACR GAPDH held in STOP buffer (endogenous kinases and phosphatases both inhibited) (Fig. 2.5). Conversely, incubations that stimulated activities of protein kinase $\mathrm{C}$ (PKC), AMP-dependent protein kinase (AMPK), or Calciumcalmodulin protein kinase (CaMK) lead to $\sim 80 \%$ decreases in ACR GAPDH activity as compared to the activity under STOP condition. Only incubation under conditions that stimulated CaMK altered the activity of LH GAPDH, the relative activity decreasing by $\sim 60 \%$ compared with STOP conditions.

\section{GAPDH Protein Expression}

Both cytoplasmic and nuclear protein levels of GAPDH were investigated in this study to determine if there were any significant changes in enzyme distribution between control and hibernating conditions. Figure 2.6 shows that GAPDH was found in both cytoplasmic and nuclear fractions. The relative amount GAPDH in the cytoplasmic compartment did not change between ACR and LH conditions and the same was true in the nuclear compartment. However, there was $\sim 20 \%$ and $\sim 40 \%$ less GAPDH expressed in the nuclear fractions from control and hibernating conditions, respectively, as 
compared to the corresponding cytoplasmic fractions, the value for LH being significantly lower.

\section{Discussion}

GAPDH is a multifunctional enzyme that plays a role in many cellular processes, with its most critical role being its central position in the glycolytic and gluconeogenic pathways within the cell. Its role in carbohydrate metabolism makes it an intriguing enzyme to investigate in animals that experience alterations in fuel metabolism due to drastic environmental changes. Thirteen-lined ground squirrels endure low-temperature torpor during their winter hibernation months and experience drastic changes to cellular metabolism, which may include reductions in both glycolytic and gluconeogenic rates (Galster and Morrison, 1970; Galster and Morrison, 1975). The present study indicates that regulation of GAPDH activity in the glycolytic versus gluconeogenic directions may contribute to the suppression of carbohydrate metabolism during torpor.

Ground squirrel muscle GAPDH was purified to electrophoretic homogeneity by using a combination of affinity and hydrophobic chromatography. The end result was an enzyme preparation that was purified 430 -fold, with a yield of $45 \%$, and a specific activity of $146 \mathrm{U} / \mathrm{mg}$ (Table 2.1; Fig 2.1). This purification scheme appears superior to that used in several other recent purifications of muscle GAPDH; camel, Camelus dromedaries, muscle GAPDH being purified with a yield of 23\% (Fourrat et al., 2007), and European pilchard, Sardina pilchardus, being purified with a yield of 25\% (Baibai et al., 2007). Thus, the purification procedure presented here indicates a novel and effective method of isolating GAPDH from a complex protein mixture. 
Kinetic analysis of GAPDH purified from euthermic control (ACR) versus deep hibernating (LH) animals revealed several differences between the two enzyme forms. Properties of the enzymes were compared at two temperatures, a high temperature $\left(36^{\circ} \mathrm{C}\right)$ that is very close to euthermic $\mathrm{T}_{\mathrm{b}}$ and a low temperature $\left(5^{\circ} \mathrm{C}\right)$, that is approximately the minimum $T_{b}$ that squirrels experience during torpor (i.e. if ambient temperature in the burrow falls to subzero values, squirrels respond with low rates of thermogenesis to keep $\mathrm{T}_{\mathrm{b}}$ above $0^{\circ} \mathrm{C}$ ). A comparison of ACR and LH GAPDH revealed a number of interesting features. At both high and low temperatures, the $\mathrm{K}_{\mathrm{m}} \mathrm{NAD}^{+}$was substantially lower for LH GAPDH as compared to ACR GAPDH (Table 2.2). Furthermore, GAPDH $\mathrm{V}_{\max }$, in both GAP-oxidizing and 1,3-BPG-reducing directions was significantly reduced for the enzyme isolated from torpid animals as compared to the enzyme from euthermic animals (Table 2.2). Since in vivo concentrations of $\mathrm{NAD}^{+}$likely meet or exceed the levels necessary for maximal GAPDH velocity (White and Schenik, 2012), it may be that the differences in $\mathrm{V}_{\max }$ are most important in assessing the functioning of GAPDH during euthermia and torpor. The decrease in LH GAPDH functionality is also corroborated by the near doubling of its activation energy as compared to the control enzyme (Table 2.3). In this respect the results for GAPDH analysis at $5^{\circ} \mathrm{C}$ do coincide with the suspected suppression of carbohydrate catabolism during torpor (cited in Buck and Barnes, 2000; Tashima et al., 1970), as well as those studies that indicate a decrease in gluconeogenic output during this same period (Zimny and Tyrone, 1957; Galster and Morrison, 1970; Galster and Morrison, 1975). This result is also supported by a previous study of skeletal muscle GAPDH from the hibernating Jerboa (Jaculus orientalis; Iddar et al., 2003), which indicated a similar decrease in GAPDH activity during torpor. Thus, the reduced 
GAPDH activity may play a role in slowing muscle glycolytic and gluconeogenic rates during torpor.

Kinetic changes in an enzyme originating from different physiological states are often indicative of structural differences between the enzymes. These variations can be confirmed through structural studies that probe the enzyme's susceptibility to denaturation/inactivation using temperature as the perturbant. Thermal denaturation of ACR and LH GAPDH indicated a distinct difference between the two enzyme forms with LH GAPDH being able to withstand incubation at higher temperatures as compared to ACR GAPDH (Fig. 2.2; Table 2.3). This resistance to thermal denaturation by GAPDH from the hibernating ground squirrel was similar to that seen by Iddar et al. (2003), who investigated purified skeletal muscle GAPDH from the hibernating Jerboa (Jaculus orientalis). These thermal denaturation studies confirm that skeletal muscle GAPDH from the torpid animal is structurally distinct from the enzyme from the euthermic condition.

Given that GAPDH is well known to be present as a single isozyme, the above structural differences between ACR and LH GAPDH are likely due to some posttranslational modification of the protein. Mass spectrometric analysis of purified GAPDH extracts revealed numerous posttranslational modifications on several different peptides in both euthermic and torpid conditions. Modifications included phosphorylation, acetylation, methylation, and oxidation, with several of the peptides retrieved being differentially modified between euthermic and torpid conditions (Table 2.4). One of the more intriguing posttranslational modifications identified was lysine acetylation. Mass spectrometric analyses showed acetylation of K213 and K217 in LH 
GAPDH but not in the identical peptides originating from ACR GAPDH. Interestingly, acetylation of plant (Arabidopsis thaliana) GAPDH on the lysine residue analogous to ground squirrel K213 was responsible for decreasing GAPDH activity, similar to the decrease seen in this investigation (Finkemeier et al., 2011). Acetylation has also been shown to mediate directionality of this enzyme within bacteria (Salmonella enterica) increased acetylation favoring glycolysis and deacetylation favoring gluconeogenesis (reviewed in Guan and Xiong, 2011; Guarente; 2011). This study was corroborated in human tumor cells with increased acetylation being linked to GAPDH activation in the glycolytic direction (Li et al., 2014). While GAPDH activity did change between ACR and LH GAPDH in this study (evident by changes in $\mathrm{V}_{\max }$; Table 2.2), it is unknown whether the potential site-specific changes in GAPDH acetylation shown here are the underlying cause. Global acetylation levels did not change between ACR and LH conditions and thus cast some doubt as to the importance of this modification in regulating GAPDH activity during torpor. Future studies will need to be focused on modifying GAPDH acetylation through incubation with commercial deacetylases and determining changes in GAPDH activity.

There are several alternative outcomes to GAPDH acetylation including alterations in GAPDH protein-protein interactions (Choudhary et al., 2009) and nuclear translocation (Ventura et al., 2010). While the former was not investigated in this study, analysis of the relative nuclear levels of GAPDH between ACR and LH indicated that GAPDH was not present in differing amounts between euthermic and torpid conditions (Fig. 6). While this coincides with the fact that global acetylation levels do not change between ACR and LH conditions, it does not indicate whether the presence of GAPDH in 
the nucleus is the result of its acetylation. Future studies would need to focus on determining the acetylation level of nuclear GAPDH in comparison to cytoplasmic GAPDH to assess if this posttranslational modification mediates GAPDH translocation. Furthermore, to investigate the potential for GAPDH acetylation to affect protein-protein interactions, co-immunoprecipitations could be used in conjunction with commercial deacetylases to determine if changes in GAPDH acetylation alters the network of proteins to which GAPDH may be bound.

Another potentially critical posttranslational modification found through mass spectrometry was GAPDH phosphorylation. Mass spectrometric analysis of ACR GAPDH revealed one peptide that contained a serine residue that was phosphorylated (S310), which was not found to be phosphorylated in the identical peptide from LH GAPDH (Table 2.4). This site has been identified previously as a phosphorylated serine residue in cancerous human T-lymphocyte GAPDH but no specific phenotypic effect has yet been associated with it (Mayya et al., 2009). To more generally assess the state of GAPDH phosphorylation, ProQ Diamond phosphoprotein staining and Western blot analyses were utilized to assess global phospho-serine, -threonine, and -tyrosine levels on euthermic versus torpid GAPDH. Results indicated that only phospho-tyrosine levels were altered on LH GAPDH as compared with ACR (i.e. 35\% lower than in ACR GAPDH) (Fig. 2.3). In an effort to determine if differential tyrosine phosphorylation regulates GAPDH activity, crude ACR and LH extracts were incubated under conditions that would stimulate endogenous protein tyrosine phosphatases and maximum GAPDH activity was reassessed. These results indicated that differential tyrosine phosphorylation does not seem to regulate GAPDH activity in vitro (Fig. 2.5). GAPDH tyrosine 
phosphorylation has been previously reported in rabbit muscle but no enzymatic effect was associated with this phosphorylation event (Sergienko et al., 1992). Bioinformatic analysis of potential phosphorylation motifs within the GAPDH primary amino acid sequence did, however, indicate a phosphorylation motif that matched the insulin receptor kinase conserved motifs, possibly substantiating some link between GAPDH regulation by tyrosine phosphorylation and fuel metabolism (Obenauer et al., 2003).

Given that some indication of differential serine phosphorylation for GAPDH was observed through mass spectrometry and that GAPDH serine/threonine phosphorylation is a well known as an important regulatory mechanism (Kawamoto and Caswell, 1986; Choudhary et al., 2000; Tisdale, 2002, Singh et al., 2004, Tisdale and Artalejo, 2007, Boulatnikov et al., 2008, Baba et al., 2010; Huang et al., 2011), additional incubations were conducted that activated specific serine/threonine protein kinases and phosphatases, prior to reassessing GAPDH activity. Incubations that stimulated exogenous alkaline phosphatase caused a significant increase in ACR GAPDH activity, while activation of endogenous PKC, AMPK, and CaMK caused significant decreases in ACR GAPDH activity (Fig. 2.5). These results suggest that LH GAPDH is present in a higherphosphorylated form in comparison to ACR GAPDH, which is contrary to the mass spectrometry results. Clearly, alterations to serine/threonine phosphorylations of GAPDH have a significant effect on GAPDH activity but this study was unable to decipher if these posttranslational modifications were key to regulating ground squirrel skeletal muscle GAPDH during torpor. To accomplish this an in-depth look at site-specific phosphorylation events would have to be undertaken. 
GAPDH methylation and dimethylation were the most prevalent modifications found through MS/MS analyses, and many sites found were novel (monomethylation E77, E170, D242, D313, E315, and dimethylation - N147, N153, N203, N237, R246; Seo et al., 2007). Comparison of the peptides from ACR and LH conditions indicates that only methylation on D313 and dimethylation on N147 and N153 on ACR GAPDH were not found on LH GAPDH (Table 2.4). Anti-methyl-asparagine, -aspartate, -glutamate, or -arginine antibodies were unavailable for Western blot analyses to further investigate the global methylation status of GAPDH between euthermia and torpor. Thus, based on these results, GAPDH methylation may be a significant regulator of this enzyme during dormancy. Western blot analyses of methyl-lysine levels indicated that indeed GAPDH was methylated but that there was no significant difference in the degree of GAPDH methylation between control and torpid states (Fig. 2.4). Again, this may a case where investigation into the effects of site-specific methylation events would be necessary to tease out the role of GAPDH methylation during torpor.

An unexpected modification to GAPDH structure was observed in one MS/MS peptide, and that was the oxidation of M229 on ACR GAPDH (Table 2.4). While this may by a result of oxidative damage to this peptide during sample processing, it is interesting to note that methionine oxidation is thought to be a form of enzyme regulation, with methionine oxidases and reductases mediating the structural changes (Stadtman et al., 2003). While this modification was not assessed via Western blots, AAPH, a free radical generator, was used to determine if ACR or LH GAPDH were more vulnerable to oxidants. These experiments revealed no difference in susceptibility of the enzymes derived from euthermic or torpid skeletal muscle tissues to AAPH-mediated 
oxidation (Table 2.3). This could suggest that methionine oxidation may be the result of deliberate action of methionine oxidases on GAPDH, however, further experiments would need confirm the prevalence of such a modification as well as the relevance to GAPDH function during torpor.

This study of 13-lined ground squirrel muscle GAPDH has indicated that the stable suppression of GAPDH activity during torpor coincides with the decrease in glycolytic and gluconeogenic output likely observed in this tissue during dormancy. The mechanism by which GAPDH enzyme activity is stably suppressed was not conclusively elucidated in this study but is likely the result of reversible phosphorylation, acetylation, or methylation of the protein. Further investigation into the role of GAPDH posttranslational modifications is crucial to understanding the regulation of this ubiquitous enzyme in the hibernating 13-lined ground squirrel. 
Table 2.1. Purification scheme for 13-lined ground squirrel ACR GAPDH.

\begin{tabular}{llllll} 
Purification Step & $\begin{array}{l}\text { Total } \\
\text { Protein } \\
(\mathrm{mg})\end{array}$ & $\begin{array}{l}\text { Total } \\
\text { Activity } \\
(\mathrm{U})\end{array}$ & $\begin{array}{l}\text { Specific } \\
\text { Activity } \\
(\mathrm{U} / \mathrm{mg})\end{array}$ & $\begin{array}{l}\text { Fold } \\
\text { Purification }\end{array}$ & \% Yield \\
\hline Supernatant & 14.4 & 4.9 & 0.34 & - & - \\
Blue Agarose & 1.4 & 5.0 & 3.6 & 10 & 100 \\
Phenyl Agarose & 0.015 & 2.2 & 146 & 430 & 45
\end{tabular}


Table 2.2. Kinetic analysis of ACR and LH GAPDH in the GAP-oxidizing and 1,3BPGreducing directions at $5^{\circ} \mathrm{C}$ and $36^{\circ} \mathrm{C}$.

\begin{tabular}{lll|ll} 
& \multicolumn{2}{l|l}{$36^{\circ} \mathrm{C}$} & \\
& & & & \\
& ACR & LH & ACR & LH \\
\hline $\mathrm{K}_{\mathrm{m}} \mathrm{GAP}(\mathrm{mM})$ & $0.45 \pm 0.02$ & $0.52 \pm 0.07$ & $1.4 \pm 0.2$ & $1.5 \pm 0.2$ \\
$\mathrm{~K}_{\mathrm{m}} \mathrm{NAD}(\mathrm{mM})$ & $1.3 \pm 0.2$ & $0.47 \pm 0.06^{*}$ & $1.3 \pm 0.3$ & $0.5 \pm 0.1^{*}$ \\
$\mathrm{~K}_{\mathrm{m}}$ 3PG (mM) & $0.22 \pm 0.03$ & $0.28 \pm 0.03$ & $0.24 \pm 0.02$ & $0.24 \pm 0.03$ \\
$\mathrm{~V}_{\max }(\mathrm{U} / \mathrm{mg} ; \mathrm{GAP}$ & $30 \pm 5$ & $11.4 \pm 0.9^{*}$ & $150 \pm 18$ & $50 \pm 2^{*}$ \\
oxidation) & & & & \\
$\mathrm{V}_{\text {max }}$ (U/mg; 3PG & $32 \pm 4$ & $18 \pm 2^{*}$ & $155 \pm 5$ & $130 \pm 13$ \\
Reduction) & & & &
\end{tabular}

Data are means $\pm \mathrm{SEM}, \mathrm{n} \geq 4$ independent determinations on separate enzyme preparations. $\mathrm{K}_{\mathrm{m}}$ values were determined at optimal co-substrate concentrations (as indicated in Materials and Methods). * indicates that the LH value is significantly different from the control (ACR) value at the same temperature as determined using the Student's t-test, $\mathrm{p}<0.05$. 
Table 2.3. Physical properties of skeletal muscle GAPDH from euthermic and torpid 13lined ground squirrels.

\begin{tabular}{lll} 
& ACR & LH \\
\hline Activation Energy $(\mathrm{kJ} / \mathrm{mol})$ & $28 \pm 2$ & $54 \pm 2^{*}$ \\
$\mathrm{I}_{50}$ AAPH $(\mathrm{mM})$ & $25 \pm 6$ & $25 \pm 3$ \\
$\mathrm{~T}_{\mathrm{m}}\left({ }^{\circ} \mathrm{C}\right)$ & $54.7 \pm 0.1$ & $56.1 \pm 0.2^{*}$
\end{tabular}

Data are means \pm SEM, $\mathrm{n} \geq 4$ independent determinations on separate enzyme preparations. Activation energy was measured for the 1,3BPG-reducing reaction of GAPDH. I $_{50}$ values were determined for the GAP-oxidizing reaction at optimal substrate concentrations (as indicated in Materials and Methods). * - indicates that the LH value is significantly different from the control (ACR) value as determined using the Student's ttest, $\mathrm{p}<0.05$. 
Table 2.4. The peptides detected by mass spectrometry in ground squirrel muscle GAPDH from ACR versus LH conditions. $\mathrm{ACR}$

70-KSITIFQERD-79
70-KSITIFQE(Me)RDPANIKW-85
143-KIVSNASCTTNCLAPLAKV-161
143-KIVSNASCTTN(Dimeth)CLAPLAKV-16
143-KIVSN(Dimeth)ASCTTNCLAPLAKV-16
198-RGAAQNIIPASTGAAKA-214
217-KVIPELNGKL-226
225-KLTGM(Ox)AFR-232
232-RVPTPNVSVVDLTCRL-247
232-RVPTPNVSVVDLTCR(Dimeth)L-247
232-RVPTPNVSVVD(Me)LTCRL-247
232-RVPTPN(Dimeth)VSVVDLTCRL-247
261-KQASEGPLKG-270
307-KLISWYDNE(Me)YGYSNRV-322
307-KLIS(Phos)WYDNEYGYSNRV-322
307-KLISWYD(Me)NEYGYSNRV-322

LH

2- KVGVNGFGRI-12

59-KAENGKLVINGKI-71

143-KIVSNASCTTNCLAPLAKV-161

160-KVIHDNFGIVE(Me)GLMTTVHAITATQKT-185

198-RGAAQNIIPASTGAAKA-214

198-RGAAQNIIPASTGAAK(Dimeth)A-214

198-RGAAQN(Dimeth)IIPASTGAAKA-214

198-RGAAQNIIPASTGAA $K(A c)$ TVG $K(A c)$ V-218

217-KVIPELNGKL-226

232-RVPTPNVSVVDLTCRL-247

232-RVPTPNVSVVDLTCR(Dimeth)L-247

232-RVPTPNVSVVD $(M e)$ LTCRL-247

261-KQASEGPLKG-270

307-KLISRYDNE(Me)YGYSNRV-322 


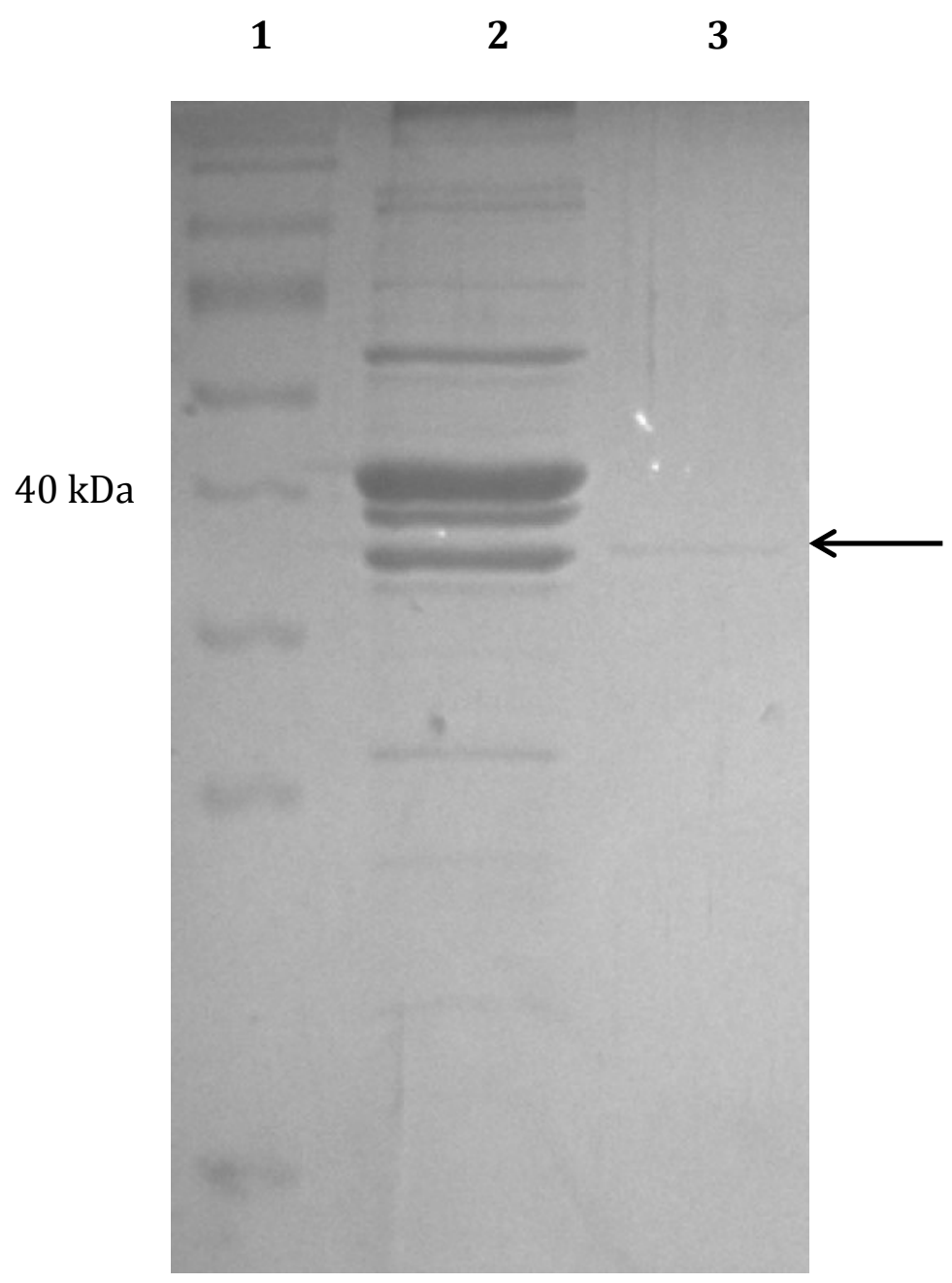

Figure 2.1 Purified ACR skeletal muscle GAPDH. The SDS-gel was stained with Coomassie Brilliant blue and the lanes show (1) Spectra Multicolour protein ladder with the $40 \mathrm{kDa}$ band indicated on the left, (2) the peak fraction of ACR GAPDH from the blue-agarose column, and (3) purified ACR GAPDH having been run through blueagarose and phenyl-agarose columns (indicated by arrow). 


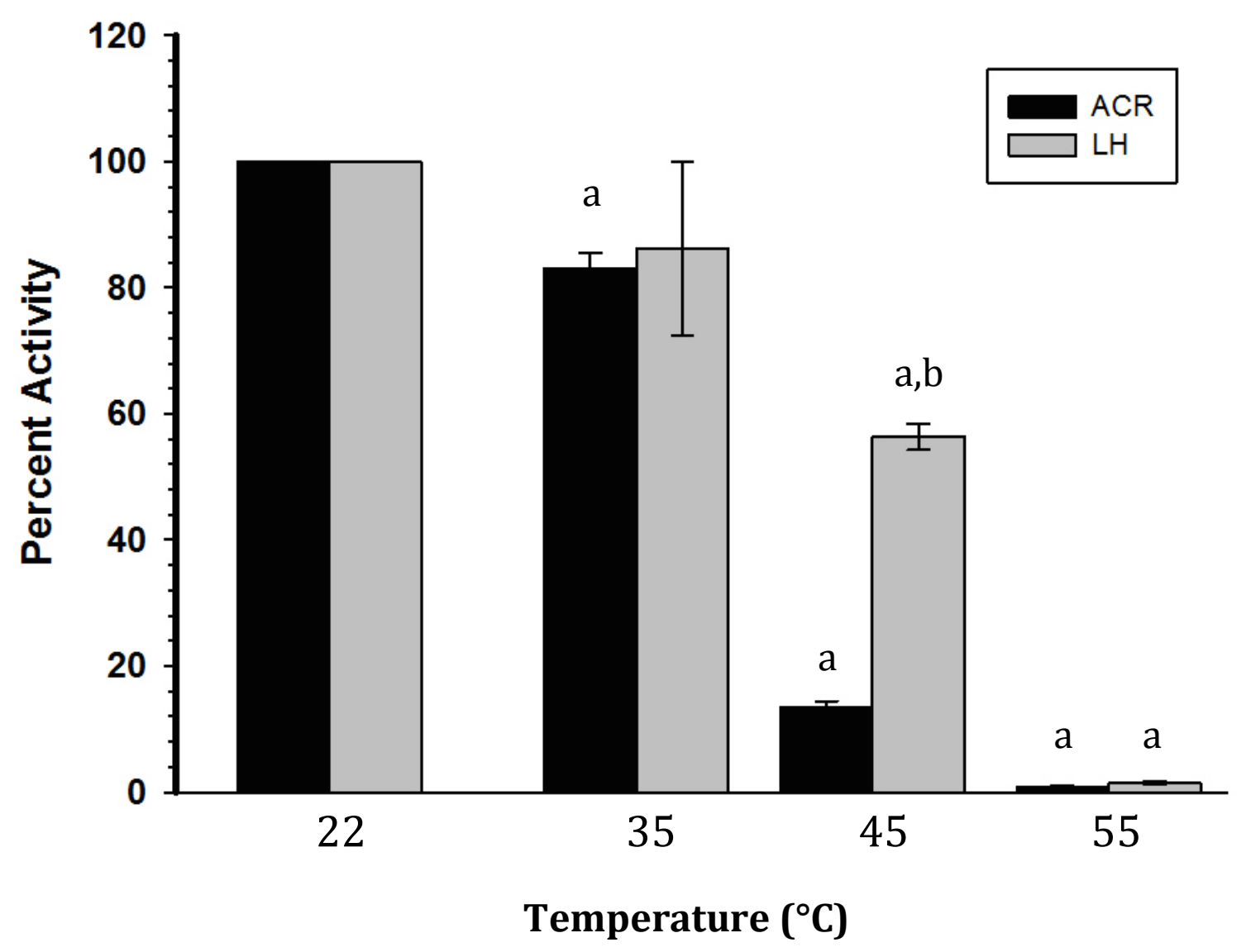

Figure 2.2 Heat-induced inactivation of ACR and LH skeletal muscle GAPDH. Data are means \pm SEM, $n=4$ independent determinations on separate enzyme preparations. a the value is significantly different from the value for the same condition assayed at $22^{\circ} \mathrm{C}$, as determined by a Holm-Sidak test, $\mathrm{p}<0.05$. b -the $\mathrm{LH}$ value is significantly different from the ACR value at the same temperature, as determined by the Holm-Sidak test, $\mathrm{p}<0.05$. 


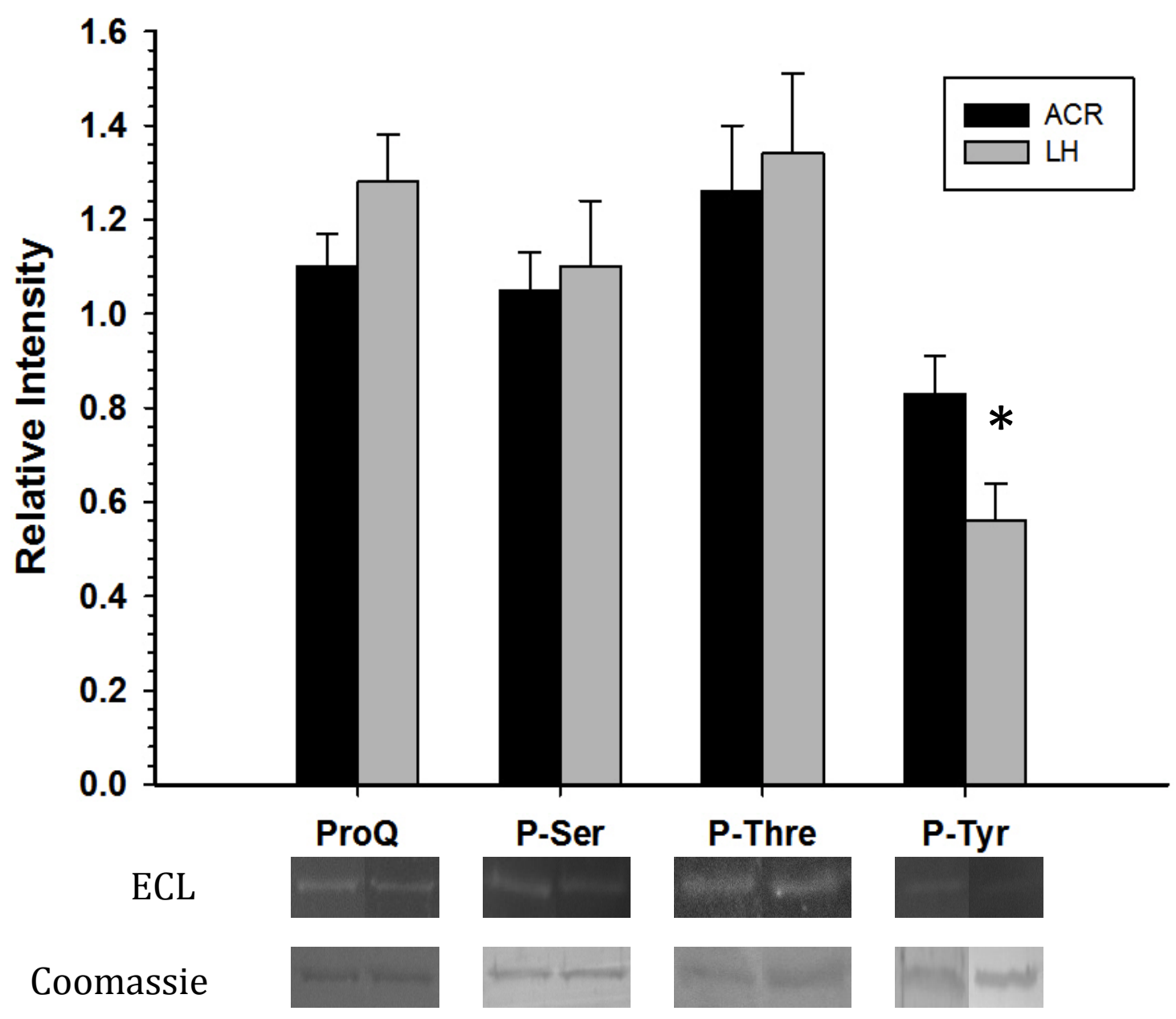

Figure 2.3 Phosphorylation state of skeletal muscle ACR and LH GAPDH: total phosphorylation as determined by ProQ Diamond phosphoprotein staining as well as relative levels of P-Ser, P-Thr and P-Tyr as determined with phospho-specific antibodies. Data are means \pm SEM, $\mathrm{n}=4$ determinations on independent enzyme samples. * _ indicates a significant difference in GAPDH phosphorylation between ACR and LH conditions using the Student's t-test, $\mathrm{p}<0.05$. 


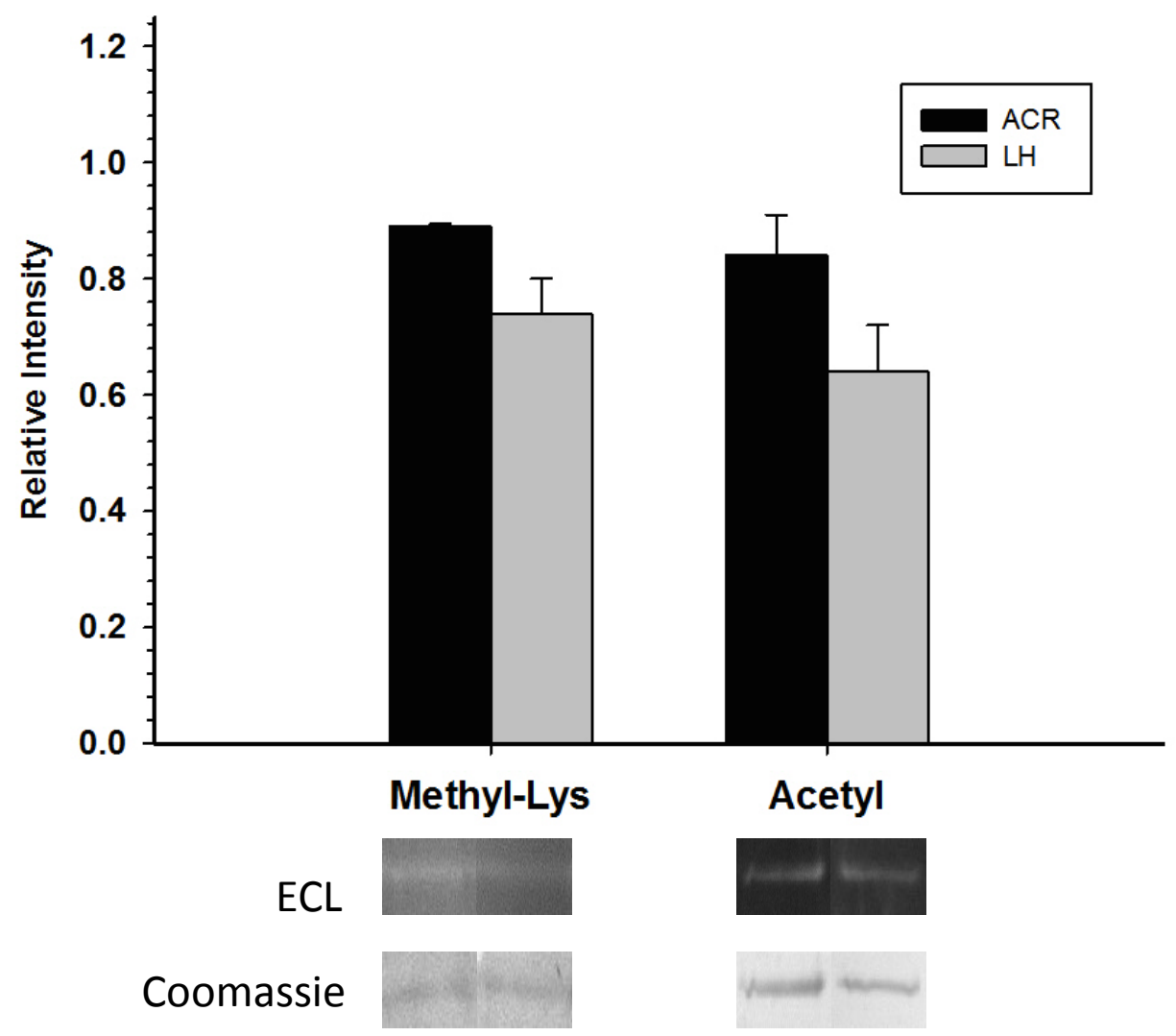

Figure 2.4 Posttranslational modification of ACR and LH skeletal muscle GAPDH:

relative changes in protein methylation and acetylation, as determined by immunoblotting. Data are means \pm SEM, $n=4$ independent samples run on the same gel. 


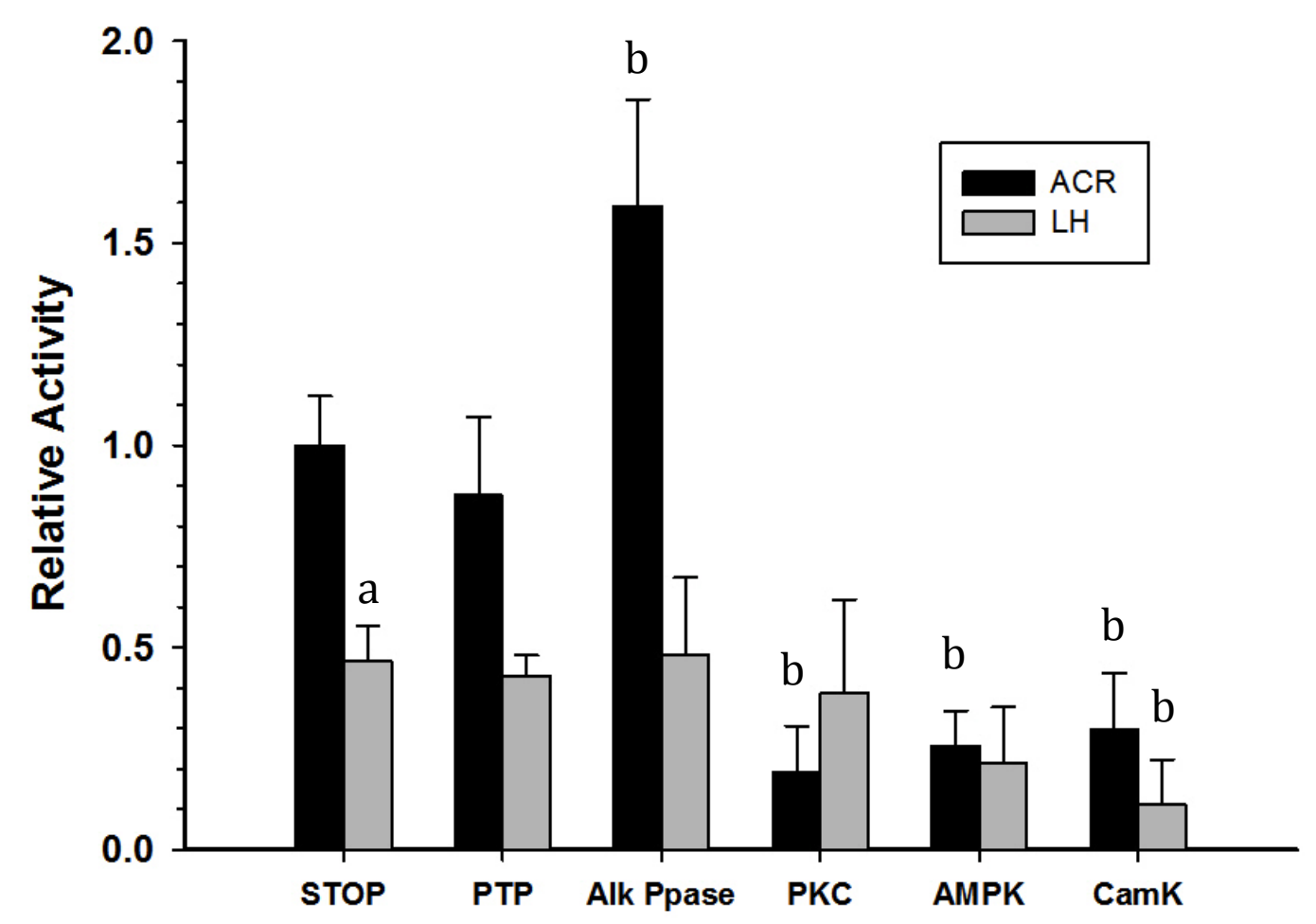

Figure 2.5 Effect of in vitro incubations that stimulate protein kinases or protein phosphatases on GAPDH activity (measured in the GAP-oxidizing direction). Data are means \pm SEM, $\mathrm{n}=3$ determinations on independent enzyme samples. ${ }^{\mathrm{a}}$ - indicates a significant difference between ACR and LH GAPDH activities under the STOP condition, as determined by the Student's t-test, $p<0.05$. $^{\mathrm{b}}$ - indicates a significant difference between the GAPDH activity value and the corresponding STOP condition, as determined by the Dunnett's test, $\mathrm{p}<0.05$. 


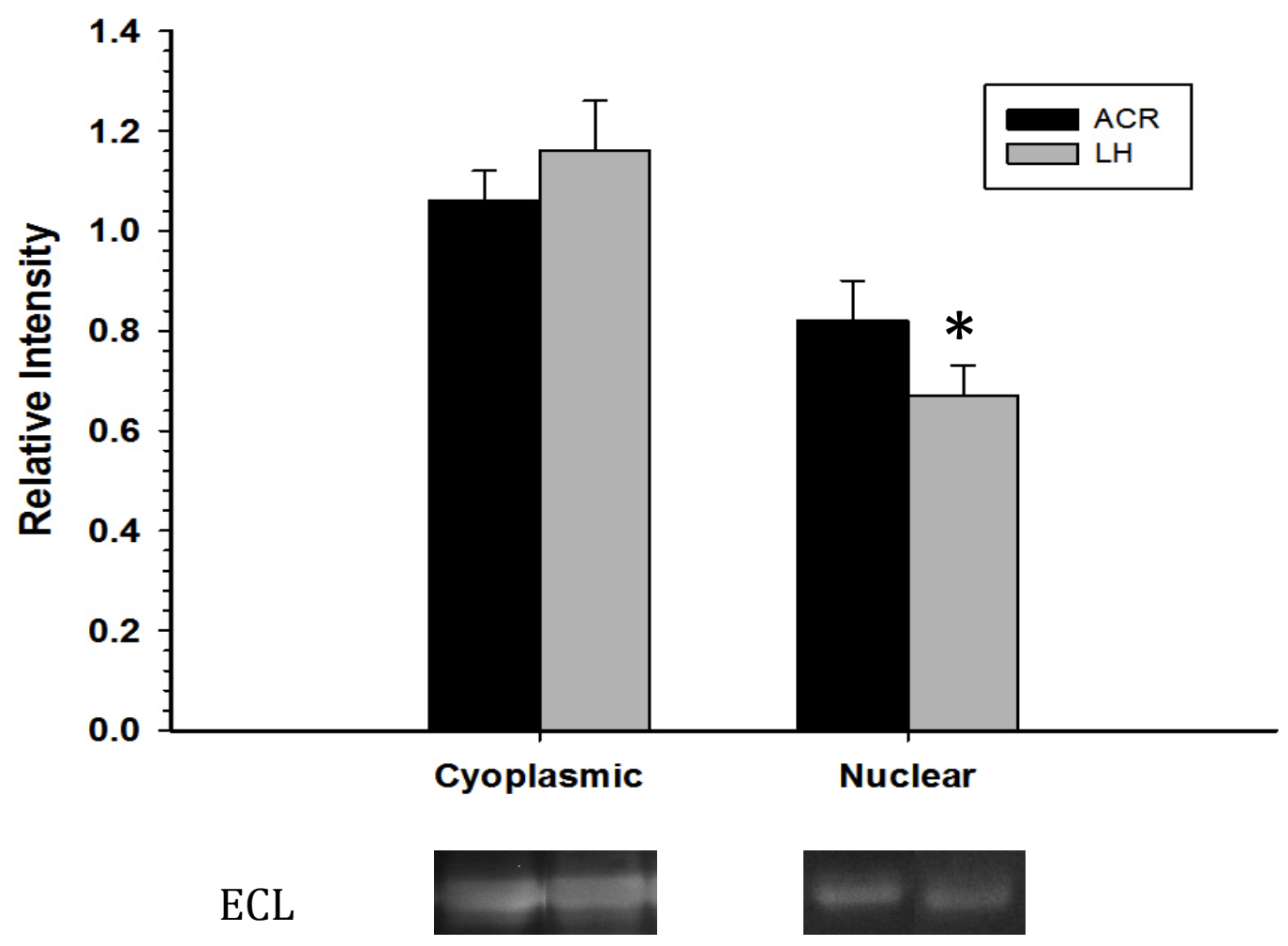

Figure 2.6 Cytoplasmic and nuclear expression of skeletal muscle GAPDH as determined by immunoblotting. Data are means \pm SEM, $n=5$ independent determinations on crude muscle extracts. * - indicates a significant difference between nuclear and cytoplasmic levels of GAPDH within a given condition, as determined by Holm-Sidak method, $\mathrm{p}<0.05$. 


\section{Chapter 3}

\section{Regulation of skeletal muscle pyruvate kinase from the hibernating ground squirrel, Urocitellus richardsonii.}




\section{Introduction}

Pyruvate kinase (PK; E.C. 2.7.1.40) is the cytosolic enzyme that catalyzes the irreversible and terminal reaction of the glycolytic pathway within mammalian cells. It mediates the transfer of the phosphate group on phosphoenolpyruvate to ADP producing ATP and pyruvate, the latter typically being shuttled into the mitochondria for further oxidation if oxygen is available (reaction is illustrated in Appendix IG). Under oxygenlimiting conditions, however, pyruvate is converted to lactate. Being at a locus of ATP production within the cell, PK is a common regulatory site in many mammalian tissues; e.g. in liver of rats (Riou et al., 1978, Ljungstrom et al., 1974) and hibernating mice (Storey, 1987) PK is regulated by reversible phosphorylation. However, this form of regulation has not been shown previously in normal (i.e. non-neoplastic) mammalian muscle cells.

Reversible phosphorylation of enzymes and functional proteins is a well-known regulatory mechanism by which the flux of cellular metabolites is controlled in response to changing environmental conditions. This is especially true during mammalian hibernation where posttranslational protein phosphorylation coordinates significant changes to cellular biochemistry during torpor bouts. This includes reversible suppression of the cell cycle (Wu and Storey, 2012a), protein synthesis (Wu and Storey 2012b), and ion transport (MacDonald and Storey, 1999). Moreover, reversible enzyme phosphorylation has been found to be critical to the regulation of metabolic processes during ground squirrel torpor. For instance, many tissues utilize reversible phosphorylation to suppress key enzymes involved in glucose metabolism as a part of the transition to non-carbohydrate fuels during dormancy (cited in Buck and Barnes, 2000; 
Tashima et al., 1970; Brooks and Storey, 1991). With respect to mammalian muscle, very few enzymes of carbohydrate metabolism have been found to be controlled by reversible phosphorylation. Exceptions include evidence of phospho-regulation of phosphoglucomutase (Hindle et al., 2011), and hexokinase (Abnous and Storey, 2008) during mammalian torpor. This study proposes that skeletal muscle PK from the hibernating ground squirrel, Urocitellus richardsonii, is regulated by reversible phosphorylation during torpor, and that increased PK phosphorylation decreases PK functioning.

\section{Materials and Methods}

Animals

Richardson's ground squirrels were captured in the summer near Calgary, Alberta and held in an animal care facility at the University of Calgary. The squirrels were housed at $22^{\circ} \mathrm{C}$ in separate cages and exposed to a fall photoperiod (10 h light: $14 \mathrm{~h}$ dark) for an 8-week feeding period to optimize their body lipid reserves. Following this time, some squirrels were removed from their cages and placed in a cold room (maintained at $\left.4^{\circ} \mathrm{C}\right)$ without food but with access to water. This room was held in a state of constant darkness for the duration of the experiments. Squirrels were allowed to enter hibernation and after one month of torpor cycles, they were then sampled in their last cycle after a minimum of 2 days of constant torpor (rectal temperature $6 \cdot 4-7 \cdot 4^{\circ} \mathrm{C}$ ). The minimum length of continuous torpor was confirmed visually by monitoring the disturbance of sawdust bedding. Euthermic squirrels, which were fed and maintained at a room temperature of $22^{\circ} \mathrm{C}$, remained alert and active prior to sampling and had rectal temperatures between $36-37^{\circ} \mathrm{C}$. At the time of sampling, all squirrels were decapitated 
and tissues were removed and frozen in liquid nitrogen. Samples were then sent to Carleton University on dry ice where they were subsequently stored at $-80^{\circ} \mathrm{C}$. PK Purification

Frozen skeletal muscle samples were homogenized 1:5 w:v using a Polytron homogenizer in buffer A (25 mM MES pH 6, 1.25 mM EDTA, 1.25 mM EGTA, 12.5 $\mathrm{mM} \beta$-glycerophosphate ( $\beta$-GP), $5 \mathrm{mM} \beta$-mercaptoethanol, and 5\% v:v glycerol). Prior to homogenization a few crystals of phenylmethylsulphonyl fluoride (PMSF) were added to inhibit serine/threonine proteases. Homogenates were centrifuged at $13,500 \mathrm{xg}$ at $5^{\circ} \mathrm{C}$, after which the supernatant was decanted and held on ice.

A $2 \mathrm{~mL}$ aliquot of supernatant was then applied to a column of carboxymethyl (CM) Sephadex (6 x $1.8 \mathrm{~cm}, \mathrm{~h} \times \mathrm{d})$ that was previously equilibrated in $15 \mathrm{~mL}$ of buffer A. After allowing the extract to seep into the column, the column was washed with 30 $\mathrm{mL}$ of buffer A and fractions were collected using a Gilson FC203B Fraction Collector. An endpoint spectrophotometric reading of the wash fractions at $280 \mathrm{~nm}$ indicated that this was sufficient washing to clear the column of any unbound protein. PK was then eluted using a $0-1 \mathrm{M} \mathrm{KCl}$ gradient in buffer A. Fractions of $\sim 0.5 \mathrm{~mL}$ were collected and assayed for PK activity. The four most active fractions were pooled $(\sim 2 \mathrm{~mL})$ and applied to a similarly sized column of blue-agarose beads pre-equilibrated in buffer B $(25 \mathrm{mM}$ imidizole, $\mathrm{pH}$ 7, $1.25 \mathrm{mM}$ EDTA, $1.25 \mathrm{mM}$ EGTA, $12.5 \mathrm{mM} \beta$-GP, $5 \mathrm{mM} \beta$ mercaptoethanol, and 5\% glycerol). After allowing the pooled fractions to seep into the blue-agarose column, the beads were washed with $30 \mathrm{~mL}$ of buffer B. Again, this was a sufficient amount of washing to rid the column of unbound protein, as determined by 
assay at $280 \mathrm{~nm}$. PK was again eluted with a $0-1 \mathrm{M} \mathrm{KCl}$ gradient, this time in buffer B, and fractions of $\sim 0.5 \mathrm{~mL}$ were collected and subsequently assayed under $\mathrm{V}_{\max }$ conditions. PK Assay

PK activity was monitored spectrophotometrically at $340 \mathrm{~nm}$ using a Thermo Labsystems Multiskan spectrophotometer. The optimal conditions for muscle PK were 1 mM phosphoenolpyruvate (PEP), 2 mM ADP, $0.15 \mathrm{mM}$ NADH, 1 Unit of lactate dehydrogenase (Sigma), $5 \mathrm{mM} \mathrm{MgCl} 2,50 \mathrm{mM} \mathrm{KCl}$, and $50 \mathrm{mM}$ imidizole, $\mathrm{pH}$ 7. Lactate dehydrogenase was added in excess to each reaction well as was determined by separate assays that measured LDH activity alone. In general, aliquots of column fractions or of the final purified enzyme were diluted 10X in buffer B before assay to obtain an appropriate amount of enzyme activity to characterize PK activity or kinetic properties. For purified PK, reactions were initiated by adding $5 \mu \mathrm{L}$ of diluted PK into a total volume of $200 \mu \mathrm{L}$. This amount of enzyme produced linear Abs vs. time curves and ensured measurement of initial reaction rates. Enzyme samples did not lose significant amounts of activity for up to two weeks (data not shown). All experiments were done within this time period.

$\mathrm{K}_{\mathrm{m}}$ values for substrates were determined at optimal co-substrate concentrations, as above. $K_{a}$ and $I_{50}$ values associated with the effects of various metabolites and salts (Mg-ATP [1:1 mix], alanine, aspartate, fructose-1,6-bisphosphate, $\mathrm{MgCl}_{2}$ and $\mathrm{KCl}$ ) on PK activity were determined at suboptimal co-substrate concentrations (0.05 mM PEP, $0.3 \mathrm{mM}$ ADP, $0.15 \mathrm{mM}$ NADH and $1 \mathrm{U}$ LDH activity).

Enzyme activity and kinetics were analyzed with a Microplate Analysis and Kinetics v. 3.5.1 programs as per Chapter 2. Likewise, the Coomassie blue dye-binding 
method was performed as described in Chapter 2.

Temperature Effects on PK

For assays at $5^{\circ} \mathrm{C}$ and $36^{\circ} \mathrm{C}$ the same procedure was followed as described in Chapter 2. For assays at $5^{\circ} \mathrm{C}, 10 \mu \mathrm{L}$ of purified (undiluted) PK was quickly added to the appropriate microplate wells to begin the assay whereas for assays at $36^{\circ} \mathrm{C}, 5 \mu \mathrm{L}$ of $30 \mathrm{X}$ diluted purified PK was added to each well (PK was held at room temperature prior to the assay). Room temperature (RT) assays were conducted at $22^{\circ} \mathrm{C}$.

The thermal stability of muscle PK was also assessed as described for 13-lined ground squirrel GAPDH in Chapter 2.

Physical Stability of PK

Investigation into the physical stability of PK from euthermic and hibernating conditions was undertaken by exposing purified PK to various concentrations of known denaturants, urea $(0-2 \mathrm{M})$ and guanidine hydrochloride $(0-0.75 \mathrm{M})$. Purified extracts were incubated with the denaturants (made in $50 \mathrm{mM}$ imidazole buffer) for $24 \mathrm{~h}$ prior to assaying for maximal PK activity.

\section{ProQ Diamond Phosphoprotein Staining}

To investigate the total phosphorylation of PK in euthermic versus hibernating states, an aliquot of the most active fraction from the blue-agarose column was mixed 1:1 v:v with SDS loading buffer (recipe defined in Chapter 2) and boiled for 5 minutes, cooled on ice and frozen at $-20^{\circ} \mathrm{C}$ until use. Aliquots were then loaded into the wells of a $10 \%$ SDS-PAGE gel and run as described in Chapter 2. The resulting gel was fixed, stained with ProQ diamond phosphoprotein stain and washed as per Chapter 2, Band intensities from ProQ staining were quantified and then the gel was then stained for 30 
min with Coomassie blue (25\% w/v Coomassie Brilliant Blue R in 50\% v/v methanol, $7.5 \% \mathrm{v} / \mathrm{v}$ acetic acid) and destained for $10 \mathrm{~min}$ with destaining mix $(60 \% \mathrm{v} / \mathrm{v}$ methanol, $20 \% \mathrm{v} / \mathrm{v}$ acetic acid in $\mathrm{ddH}_{2} \mathrm{O}$ ). The location of PK subunits was identified by comparison with the position of commercial purified rabbit muscle PK (Sigma) that was loaded into one lane of the gel. PK band intensities from the ProQ Diamond stain were normalized against the corresponding intensities of the same band when stained with Coomassie brilliant blue.

\section{Immunoblotting}

Western blots were prepared and run as described in Chapter 2. Following protein transfer, the PVDF membranes were incubated overnight at $4^{\circ} \mathrm{C}$ with either: (1) Pan-acetyl primary antibody (Santa Cruz Biotechnology), (2) Phospho-serine primary antibody (Calbiochem), or (3) Phospho-tyrosine primary antibody (Cell Signaling Technology). All primary antibodies were diluted 1:1000 v:v in TBST and all secondary antibodies were diluted 1:4000 v:v in TBST as described in Chapter 2. Washing and chemiluminescent detection were carried out as per the previous chapter.

\section{Alkaline Phosphatase Incubations}

Crude skeletal muscle samples were incubated 1:1 v:v in phosphatase incubation buffer (50 mM imidizole, $\mathrm{pH}$ 7, 10\% v:v glycerol, $10 \mathrm{mM} \beta$-mercaptoethanol, $20 \mathrm{mM}$ $\mathrm{MgCl}_{2}, 10 \mathrm{mM}$ EDTA, and 15 Units of alkaline phosphatase) for $24 \mathrm{~h}$ at $4^{\circ} \mathrm{C}$. Following the incubation, PK was purified as outlined above and an aliquot of the purified enzymes from euthermic and hibernating conditions were diluted 2:1 v:v in SDS loading buffer, boiled for 5 minutes and stored at $-20^{\circ} \mathrm{C}$. These were subsequently used for ProQ Diamond phosphoprotein stain analysis (details above and in Chapter 2). The remaining 
enzyme was assayed to determine the $\mathrm{K}_{\mathrm{m}} \mathrm{ADP}$ at room temperature.

\section{Bioinformatics}

Posttranslational modification predictor sites were used to corroborate the data obtained experimentally in this study. To utilize these predictor sites the PK amino acid sequence was obtained for the thirteen-lined ground squirrel, as opposed to the Richardson's ground squirrel as this animal is unsequenced. The thirteen-lined ground squirrel PK sequence was obtained from the Broad Institute (http://www.ensembl.org/Spermophilus_tridecemlineatus /Info/Index) and is shown in Appendix IC. This sequence was input into the NetPhos 2.0 server (Blom et al., 1999) for the identification of possible phosphorylation sites.

\section{Results}

PK Purification

PK was purified from Richardson's ground squirrel skeletal muscle through the use of an ion exchange column ( $\mathrm{CM}^{-}$Sephadex) and an affinity column (blue-agarose). PK was purified 390 fold, with an overall yield for the final PK preparation of $21 \%$ and a specific activity of $390 \mathrm{U} / \mathrm{mg}$ (Table 3.1; Fig. 3.1).

\section{Purified PK Kinetics}

Purified squirrel skeletal muscle PK displayed markedly different kinetics between euthermic and torpid states. One distinct change in kinetics was in the $\mathrm{K}_{\mathrm{m}} \mathrm{PEP}$, where torpid PK displayed a significantly higher $\mathrm{K}_{\mathrm{m}}$ PEP at all three assay temperatures as compared to the euthermic condition (Table 3.2). Torpid PK displayed its highest $\mathrm{K}_{\mathrm{m}}$ PEP at the temperature closest to $\mathrm{T}_{\mathrm{b}}$ during euthermia, while euthermic $\mathrm{PK} \mathrm{K}_{\mathrm{m}} \mathrm{PEP}$ remained relatively constant at all assay temperatures. Conversely, $\mathrm{K}_{\mathrm{m}} \mathrm{ADP}$ for torpid 
PK was $61 \%$ and $54 \%$ lower at $\mathrm{RT}$ and $35^{\circ} \mathrm{C}$, respectively, as compared to the values for euthermic PK. Interestingly, torpid PK displayed its highest $K_{m}$ ADP at the $T_{b}$ typically found during torpor, while euthermic $\mathrm{PK} \mathrm{K}_{\mathrm{m}}$ PEP was highest at RT and lowest at the two temperature extremes $\left(5^{\circ} \mathrm{C}\right.$ and $\left.35^{\circ} \mathrm{C}\right)$. Both euthermic and torpid PK displayed predictable behaviours at the various assay temperatures, with increasing temperature leading to increased enzyme activity. However, the degree to which enzyme activity increased varied between the two conditions. Torpid $\mathrm{PK} \mathrm{V}_{\max }$ was $45 \%$ higher at $5^{\circ} \mathrm{C}$ and $\sim 50 \%$ lower at room temperature and $35^{\circ} \mathrm{C}$ as compared to the corresponding euthermic $\mathrm{V}_{\max }$ at those temperatures.

Effectors of $P K$

PK from euthermic and torpid conditions responded differently to changes in $\mathrm{pH}$ (Fig. 3.2). Euthermic $\mathrm{PK}$ was sensitive to changes in $\mathrm{pH}$ above or below neutral $\mathrm{pH} 7$, whereas torpid PK maintained an optimal activity from $\mathrm{pH} 6$ to 8 . Both enzyme forms were completely inactive at $\mathrm{pH} 10.5$.

The response of muscle PK to various metabolites commonly encountered in a cell was investigated in this study. One consistent inhibitor of PK activity was ATP, which inhibited euthermic and torpid forms of PK at sub-saturating substrate levels and at all temperatures. Torpid PK was significantly less susceptible to ATP inhibition at $5^{\circ} \mathrm{C}$ and $35^{\circ} \mathrm{C}$, with a $\sim 5$-fold and 2-fold higher $\mathrm{I}_{50} \mathrm{ATP}$ respectively, as compared to the euthermic values. Furthermore, at the same temperatures, the fold inactivation (i.e. the maximum inhibition of PK at the highest concentration of ATP tested $(10 \mathrm{mM})$ ) values for torpid PK caused by ATP were 70\% and 50\% less respectively, as compared to the values for euthermic PK (Table 3.2). 
$\mathrm{KCl}$ and $\mathrm{MgCl}_{2}$ activated $\mathrm{PK}$ from both conditions at all three temperatures. PK from the torpid condition was markedly less sensitive to $\mathrm{MgCl}_{2}$ activation at $5^{\circ} \mathrm{C}$ as compared to PK from the euthermic condition, with $\mathrm{K}_{\mathrm{a}} \mathrm{MgCl}_{2}$ of $0.7 \pm 0.1 \mathrm{mM}$ and $4 \pm 1$ $\mathrm{mM}$, respectively (Table 3.2). At room temperature torpid PK displayed a significantly lower $\mathrm{K}_{\mathrm{a}} \mathrm{MgCl}_{2}$ at $1.2 \pm 0.3 \mathrm{mM}$ as compared to $\mathrm{PK}$ from the euthermic condition which showed a $\mathrm{K}_{\mathrm{a}}$ of $3.1 \pm 0.1 \mathrm{mM}$. Lastly, at $35^{\circ} \mathrm{C}$, there was no difference between euthermic and torpid $\mathrm{PK}$ responses to $\mathrm{MgCl}_{2}$. Opposite to the situation with $\mathrm{MgCl}_{2}$ at $5^{\circ} \mathrm{C}$, torpid $\mathrm{PK}$ was significantly more sensitive to $\mathrm{KCl}$ with a $\mathrm{K}_{\mathrm{a}}$ that was $69 \%$ less than the corresponding $\mathrm{K}_{\mathrm{a}}$ from the euthermic enzyme (Table 3.2). At room temperature and $35^{\circ} \mathrm{C}, \mathrm{K}_{\mathrm{a}} \mathrm{KCl}$ and the fold activation by the monovalent cation was similar for PK from both conditions.

Several different metabolites were tested to see if they would affect PK activity at suboptimal substrate concentrations. The amino acids L-alanine and L-aspartate did not affect PK activity significantly at concentrations up to $10 \mathrm{mM}$. Similarly, the Kreb cycle intermediate, citrate, did not have any effect on PK activity at concentrations up to 10 $\mathrm{mM}$. Fructose-1,6-bisphosphate also showed little propensity to effect PK activity under the condition of this experiment up to a concentration of $10 \mathrm{mM}$.

\section{Physical Stability of PK}

PK stability was assessed by exposing the purified enzyme to various concentrations of the chemical denaturants, urea and guanidine hydrochloride. At $5^{\circ} \mathrm{C}$ torpid PK was significantly less susceptible to urea denaturation as compared to the euthermic enzyme (Table 3.3). This difference in structural stability was lost at room temperature with euthermic and torpid PK displaying similar $\mathrm{I}_{50}$ urea values. Lastly, at 
$35^{\circ} \mathrm{C}$, the $\mathrm{I}_{50}$ urea could not be determined for euthermic $\mathrm{PK}$ as the purified enzyme was unstable and lost all activity after an overnight incubation at $35^{\circ} \mathrm{C}$. The torpid form of PK however, was able to withstand the prolonged exposure to higher temperatures and displayed an $\mathrm{I}_{50}$ urea of $0.60 \pm 0.03 \mathrm{M}$. It is interesting to note that torpid PK became increasingly more susceptible to urea denaturation at higher temperatures, however, euthermic PK became less susceptible to urea-induced unfolding when comparing the incubations from room temperature and $5^{\circ} \mathrm{C}$.

Guanidine hydrochloride-induced denaturation of euthermic and torpid PK was nearly identical at $5^{\circ} \mathrm{C}$ and varied only slightly at room temperature, with torpid PK displaying a 24\% higher $\mathrm{I}_{50} \mathrm{GnHCl}$. Again, euthermic PK was unable to withstand incubation at high temperature overnight and thus the $\mathrm{I}_{50} \mathrm{GnHCl}$ could not be determined at $35^{\circ} \mathrm{C}$. PK from the torpid condition, however, had an $\mathrm{I}_{50} \mathrm{GnHCl}$ of $0.13 \pm 0.01 \mathrm{M}$ at the same temperature. Similar to the situation with urea, torpid PK was most susceptible to $\mathrm{GnHCl}$ denaturation at high temperature, whereas euthermic PK became more resistant to $\mathrm{GnHCl}$-induced unfolding at room temperature as compared to $5^{\circ} \mathrm{C}$.

In addition to chemical denaturation, the physical stability of PK was assessed by thermal denaturation. As is evident in Figure 3.3, torpid PK was less stable at higher temperatures than euthermic PK. The percentage of torpid PK activity remaining after incubation at higher temperatures was significantly lower than the comparable values for euthermic PK activity at all three temperatures tested: $35^{\circ} \mathrm{C}, 45^{\circ} \mathrm{C}$, and $55^{\circ} \mathrm{C}$.

\section{Phosphorylation of $P K$}

Investigation into the phosphorylation state of squirrel muscle PK began with using ProQ Diamond phosphoprotein stain to quantify the overall level of enzyme 
phosphorylation. Figure 3.4 indicates that torpid PK was $\sim 40 \%$ more phosphorylated than euthermic PK, and that when the purified enzymes were treated with commercial alkaline phosphatase there was a dramatic decrease in protein phosphorylation. Although not statistically significant due to high variability in the data for phosphatase-treated torpid PK, dephosphorylation of torpid PK appeared to alter the kinetics of the enzyme, as is evident in Figure 3.5 where $\mathrm{K}_{\mathrm{m}} \mathrm{ADP}$ of torpid PK increased nearly 2-fold after phosphatase treatment to a value similar to that seen for euthermic PK.

Subsequent Western blot analysis indicated that torpid PK had a greater degree of serine phosphorylation, $\sim 40 \%$ more, as compared to the control condition (Fig. 3.6). Conversely, PK tyrosine phosphorylation did not change between the two conditions.

\section{Other Posttranslational Modifications}

In addition to phosphorylation, posttranslational acetylation of ground squirrel muscle PK was investigated. Figure 3.6 shows that torpid PK appears to be more highly acetylated, with a $\sim 70 \%$ greater signal detected as compared to euthermic PK.

\section{Discussion}

Entrance into torpor during winter hibernation is characterized by a strong reduction in the metabolic rate, often to values just $1-5 \%$ of normal (Geiser, 1988) and a reorganization of fuel usage within most body tissues to favour lipid oxidation (Hochachka and Guppy, 1987). Skeletal muscle metabolism is no exception, with various studies indicating significant decreases in muscle glycolytic activity during hibernation (Hachimi et al., 1990; Brooks and Storey, 1991; Abnous and Storey, 2008). Glycolytic suppression during hibernation is usually mediated through the regulation of one or more key enzymes within the pathway, and this study demonstrates that PK might 
be a key regulatory point that aids in tempering skeletal muscle glucose catabolism during torpor.

Previous studies on golden-mantled ground squirrel (Spermophilus lateralis) skeletal muscle PK did not show any differences in enzyme kinetic properties between euthermic and hibernating animals when the enzyme was analyzed in crude preparations (Brooks and Storey, 1992). While that study touched the surface of several glycolytic enzymes, this study purified skeletal muscle PK to clearly assess the detailed structural and kinetic characteristics of PK from a hibernator.

PK from hind leg skeletal muscle of Urocitellus richardsonii was purified to electrophoretic homogeneity (Fig. 3.1) through the use of $\mathrm{CM}^{-}$Sephadex and blueagarose columns. The overall yield of activity was $21 \%$, which is comparable to that of Randall and Anderson (1975) and Nishikawa et al. (2001), who purified PK from the muscle of sturgeon and lamprey, respectively. Thus, the combination of ion exchange and affinity chromatography utilized in this study is an efficient way to purify PK from complex protein mixtures.

Investigation into the behaviour of squirrel skeletal muscle pyruvate kinase began with a look into the kinetics of this enzyme at high and low temperatures. Generally speaking, regardless of temperature, ground squirrel muscle PK behaved similarly to an M1-type PK typically found in skeletal muscle (Hall and Cottam, 1978). For instance, ground squirrel PK displayed a $\mathrm{K}_{\mathrm{m}}$ PEP that ranged from 0.03-0.1 mM with a Hill coefficient of $\sim 1$ (i.e. a hyperbolic velocity versus substrate concentration relationship) (data not shown), as well as $\mathrm{K}_{\mathrm{m}} \mathrm{ADP}$ and $\mathrm{I}_{50}$ ATP that are fairly close to typical M1-type PK values (Hall and Cottam, 1978). 
Although euthermic and torpid PK displayed properties that were typical of M1type enzymes, there were significant differences between PK kinetics from the two conditions. At $35^{\circ} \mathrm{C}$, or near euthermic $\mathrm{T}_{\mathrm{b}}$, numerous kinetic changes were observed between PK isolated from euthermic versus torpid animals. These included a significantly higher $K_{m}$ PEP, and lower $K_{m} A D P$ and $V_{\max }$ for torpid PK as compared to the euthermic form of the enzyme. In deciphering the possible significance of these changes to the ground squirrel it is important to look at the endogenous levels of metabolites and substrates typically found during euthermia. Endogenous levels of ADP within resting mammalian muscle are approximately $1 \mathrm{mM}$ (English and Storey, 2000), which is well above the $\mathrm{K}_{\mathrm{m}} \mathrm{ADP}$ values for either enzyme form at $35^{\circ} \mathrm{C}$ (Table 3.1). This suggests that the decrease in $\mathrm{K}_{\mathrm{m}} \mathrm{ADP}$ for torpid $\mathrm{PK}$ at $35^{\circ} \mathrm{C}$ may be inconsequential since ADP levels are nearing saturating conditions. Conversely, mammalian skeletal muscle PEP concentrations under normal conditions range from 30-60 $\mu \mathrm{M}$ (Dohm et al., 1986; Storey and Kelly, 1995), which is well within the $\mathrm{K}_{\mathrm{m}}$ values determined in this study; this suggests that $\mathrm{K}_{\mathrm{m}}$ and in vivo PEP levels may be key for determining PK activity. Thus, the lower $\mathrm{K}_{\mathrm{m}}$ PEP values for euthermic PK as compared to torpid PK indicates that the torpid form of the enzyme is less able to function at high temperature, while the euthermic form appears suited for high temperature function.

Assays at $5^{\circ} \mathrm{C}$ also revealed significant kinetic differences between torpid and euthermic PK, however, these differences did not decidedly indicate a more or less active PK at low temperatures. Torpid PK showed a significant increase in $K_{m} P E P$ and $V_{\max }$ when compared to the euthermic enzyme measured at the same temperature (Table 3.2). Although contradictory, it is unlikely that $\mathrm{PK}$ would function at $\mathrm{V}_{\max }$ capacity since the 
muscle PEP concentration is known to decrease from the above stated range during torpor (Storey and Kelly, 1995). This suggests that the increase in $\mathrm{K}_{\mathrm{m}}$ PEP may be the more significant factor during torpor and thus may indicate a decrease in PK functioning during dormancy.

A decreased functionality for PK at the low body temperatures typically found during torpor coincides well with the overall muscle metabolic landscape found during dormancy. During torpor most organs, including the skeletal muscle, switch mainly from carbohydrate to lipid oxidation for energy production (cited in Buck and Barnes, 2000; Tashima et al., 1970; Hochachka and Guppy, 1987). While suppression of carbohydrate metabolism typically entails decreasing glycogen catabolism (through regulation of glycogen phosphorylase; Storey, 1987), and/or decreasing glucose catabolism (through regulating hexokinase; Abnous and Storey, 2008), PK typically plays an important regulatory role in slowing glycolytic rate further along the glycolytic pathway. PK as a glycolytic control point during hibernation has been almost exclusively associated with mammalian liver (Storey, 1987). However, studies in the hibernating jumping mouse, Zapus hudsonius, showed that skeletal muscle PEP concentrations rose during torpor but concentrations of pyruvate decreased during dormancy, suggesting that muscle PK may be an important control point within hibernator muscle during torpor (Kelly and Storey, 1995).

PK is typically susceptible to allosteric effects by a variety of metabolites depending on the tissue-specific isoform being studied. An important regulatory molecule for PK is ATP. At both high and low temperatures torpid PK was significantly less susceptible to ATP inhibition than euthermic PK (Table 3.3). Looking at the 
endogenous levels of ATP in muscle during euthermia $(\sim 6 \mathrm{mM})$ and hibernation $(\sim 3.5$ $\mathrm{mM}$ ) it is clear that PK from this ground squirrel may be sufficiently susceptible to ATP inhibition to make it a potentially important regulatory molecule throughout the life of the squirrel (English and Storey, 2000). The fact that euthermic PK is particularly more sensitive to ATP inhibition likely reflects the fact that at a normal $T_{b}$ energy flux can be much greater and rapid changes in ATP levels would need to temper glycolytic output more forcefully.

All isoforms of PK require monovalent and divalent cations for activity, and in vivo these cations are most likely potassium and magnesium, respectively (Larson et al., 1994; Frey and Hegeman, 2007). In this study, increasing concentrations of potassium and magnesium activated both euthermic and torpid PK, but the sensitivity to activation by these ions varied between the two enzyme forms. With regards to magnesium, torpid $\mathrm{PK}$ at $5^{\circ} \mathrm{C}$ was significantly less sensitive to the divalent cation and also showed a substantially lower fold activation as compared to euthermic PK under the same conditions (Table 3.3). Judging by the known intracellular concentration of magnesium within skeletal muscle (ranging from $\sim 0.5$ to $3 \mathrm{mM}$; MacDermott, 1990), the insensitivity of torpid PK at low temperature may contribute to decreasing PK functioning during dormancy. With respect to potassium, torpid PK displayed a significantly lower $\mathrm{K}_{\mathrm{a}} \mathrm{KCl}$ at $5^{\circ} \mathrm{C}$ as compared to euthermic PK (Table 3.3). However, the approximate potassium ion concentration within mammalian muscle is $\sim 200 \mathrm{mM}$ (Bijlani et al., 2010), and even with a slight reduction in concentration during hibernation (Willis et al., 1971), KCl levels are likely to be well above the $\mathrm{K}_{\mathrm{a}}$ values observed here. Thus, the most relevant kinetic parameter with regards to potassium would be its resulting fold activation of PK, 
which is not significantly different between the two enzyme forms at any temperature studied (Table 3.3).

Fructose-1,6-bisphosphate $\left(\mathrm{F}_{16 \mathrm{P}_{2}}\right)$ is a common allosteric regulator of most PK isozymes, however, it is not known to affect the most common skeletal muscle isozyme, PKM1. The binding site for $\mathrm{F}_{16} \mathrm{P}_{2}$ is thought to be coded for by the spliced portion of the PKM mRNA (unspliced mRNA is responsible for the production of PKM2; Jurica et al., 1998). Consistent with these facts, $\mathrm{F}_{16 \mathrm{P}_{2}}$ did not affect euthermic or torpid PK at any temperature in this study. Several other common cellular molecules were tested for their effects on PK activity, including L-alanine, L-aspartate and citrate. Alanine is a known inhibitor of the liver isozyme of $\mathrm{PK}$, but is typically not a regulatory molecule for the muscle isozyme (Carbonell et al., 1973). The results for ground squirrel PK concur support as alanine did not affect euthermic or torpid PK activity at concentrations as high as $10 \mathrm{mM}$. Alternatively, aspartate is known to activate plant-derived PK (Smith et al., 2000), as well as PK from a variety of anoxia-tolerant molluscs (Storey, 1986). However, aspartate did not affect ground squirrel muscle PK. Citrate has not previously been reported to affect PK but, it is a common product of lipid oxidation and could accumulate to appreciable amounts as hibernators favour lipid catabolism during torpor (Storey, 1989). Citrate has been shown to affect several metabolic enzymes, including phosphofructokinase (Whitwam and Storey, 1991) and glucose-6-phosphate dehydrogenase (Ramnanan and Storey, 2006) in estivating land snails, however, it does not appear to affect PK within the hibernating ground squirrel (Table 3.2).

The kinetic changes noted above suggest that there may be structural variations between the PK from the skeletal muscle of euthermic animals and PK from animals that 
are in torpor. Two common methods that can be used to elucidate structural differences between two enzyme forms are chemical and thermal denaturation. With regards to chemical denaturation, both urea and guanidine hydrochloride were used. Urea is thought to interact directly with positively charged residues on the surface of the protein as well as with amidic linkages of the peptide backbone to facilitate $\beta$-sheet unfolding and protein denaturation (Rocco et al., 2008; Almarza et al., 2009), while guanidine hydrochloride appears to mask electrostatic interactions within the protein to promote destabilization (Monera et al., 1994). Table 3.4 shows that torpid PK is significantly less susceptible to urea denaturation as compared to euthermic PK at $5^{\circ} \mathrm{C}$. Furthermore, as the incubation temperature increased, torpid PK became increasingly more susceptible to urea denaturation. Euthermic PK was less susceptible to urea-induced denaturation from $5^{\circ} \mathrm{C}$ to $\mathrm{RT}$, but was unable to be measured at $35^{\circ} \mathrm{C}$. This is somewhat surprising as it was expected that euthermic PK be more stable at higher temperatures as compared to torpid PK. This may be the result of the combinatorial effects of chemical denaturants and temperature, since denaturation using temperature alone indicated that euthermic PK was more stable at $35-40^{\circ} \mathrm{C}$ (Fig. 3.3).

In comparison to urea, $\mathrm{GnHCl}$ was a much stronger denaturant, showing $\mathrm{I}_{50}$ values 2-5 fold lower than the comparable values for urea, which is in line with previous experiments using these denaturants (Greene and Pace, 1974). The changes observed for $\mathrm{I}_{50} \mathrm{GnHCl}$ values between temperatures or metabolic states were less pronounced than those seen for urea, with torpid PK being only $7 \%$ more susceptible to denaturation at $5^{\circ} \mathrm{C}$, and $\sim 20 \%$ less susceptible to denaturation at RT (Table 3.4). Generally speaking, it appears that torpid PK is significantly more stable at low temperatures as compared to 
euthermic PK. This may be significant during torpor as protein turnover is suppressed as part of metabolic rate depression since it would be impossibly costly to try to maintain euthermic rates of protein synthesis and degradation. Thus, a more chemically stable enzyme may be able to withstand greater insult by the cellular environment without unfolding and requiring recycling.

Thermal denaturation studies were conducted by assaying purified PK under $\mathrm{V}_{\max }$ conditions following a short (15 min) incubation at a given temperature and a cooling period on ice to RT. Figure 3.3 illustrates that euthermic PK is significantly more stable at higher temperatures as compared to torpid PK, with euthermic PK maintaining a fairly stable $V_{\max }$ up to $45^{\circ} \mathrm{C}$ whereas the $\mathrm{V}_{\max }$ of torpid $\mathrm{PK}$ dropped significantly following incubation at $35^{\circ} \mathrm{C}$. This result matches the chemical stability studies presented above for torpid PK, which demonstrated that torpid PK was less stable at high temperature. The greater stability of PK derived from euthermic tissue coincides with the fact that the enzyme typically functions at approximately $35^{\circ} \mathrm{C}$, and thus should be better suited to function higher temperature than torpid PK.

Based on the kinetic and stability variations between euthermic and torpid PK it appears that there may be significant structural differences between PK from the two conditions. Structural variations in proteins are common in animals that transition into hypometabolic states, and usually occur in the form of posttranslational modifications to the proteins. Principal among these modifications is reversible protein phosphorylation, which was investigated in this study through ProQ Diamond phosphoprotein staining as well as analysis of global phospho-serine and phospho-tyrosine content of proteins via Western blots on purified PK. Figure 3.4 shows that the relative intensity of bands for 
torpid PK on an SDS-PAGE gel was $\sim 40 \%$ greater than the band intensity for euthermic PK when analyzed by ProQ Diamond phosphoprotein staining. Subjecting both enzyme forms to alkaline phosphatase treatment for $24 \mathrm{~h}$ resulted in a drastic decrease in ProQ staining, which supports the validity of the previous ProQ Diamond results. Furthermore, phospho-serine Western blots showed that torpid PK contained $\sim 50 \%$ more phosphorylated serine residues than PK from the control condition (Fig. 3.6). Conversely, phospho-tyrosine levels remained relatively constant between euthermic and torpid conditions.

Pyruvate kinase has been known to be phosphorylated in a variety of systems, such as rat, chicken and human liver (Titanji et al., 1976; Riou et al., 1978, Boivin et al., 1980; Fister et al., 1983), rat pancreatic islet (MacDonald and Kowluru, 1985), human and rat erythrocyte cells (Nakashima et al., 1982), and many human cancer cells (Weernink et al., 1990; Hitosugi et al., 2009). Phosphorylation of muscle PK is far less common, and has only been found in the channelled whelk and the spadefoot toad (Plaxton and Storey, 1984; Cowan and Storey, 1999). The only experimental evidence for mammalian muscle PK phosphorylation comes from work on human cancer cells, which showed increased phosphorylation of pyruvate kinase muscle isoform 2 (PKM2). Although this isoform is not found in mammalian skeletal muscle it has an amino acid sequence that is only $4 \%$ dissimilar to the dominant muscle isoform (i.e. PKM1). Indeed, PKM1 and PKM2 are expressed from alternative RNA splicing of the same gene (Noguchi et al., 1986), and the 43 extra amino acids that are present in PKM2 do not appear to contain any obvious phosphorylation motifs (Weernink et al., 1990). Furthermore, computational analysis of mammalian muscle PK does show considerable 
evidence for serine, threonine and tyrosine phosphorylation sites on 13-lined ground squirrel muscle PK as shown by the NetPhos 2.0 output (Blom et al., 1999; Appendix ID). Thus, it does appear possible that mammalian muscle PK can be phosphorylated and this is the first study that shows this to be true.

The known effects of reversible phosphorylation on mammalian PK functioning are inconsistent, with some reports indicating that it has no affect on PK activity (MacDonald and Chang, 1985), and others showing phosphorylation inhibits PK (Eigenbrodt et al., 1977; Riou et al., 1978; Presek et al., 1988; Hitosugi et al., 2009). In an effort to determine whether reversible PK phosphorylation had an effect on enzyme function in this study, crude muscle extracts were incubated with exogenous alkaline phosphatase prior to purification and the $\mathrm{K}_{\mathrm{m}}$ ADP was reassessed following purification. Figure 3.5 shows that prior incubation of euthermic PK with alkaline phosphatase did not affect its affinity for ADP to a significant degree, however, incubation of torpid PK with alkaline phosphatase increased the $\mathrm{K}_{\mathrm{m}} \mathrm{ADP}$ by approximately 2-fold to a value similar to that seen for euthermic PK. Thus, it appears that reversible phosphorylation may regulate changes in PK kinetics when the animal transitions into torpor.

In addition to reversible phosphorylation, posttranslational acetylation was investigated in this study to discern if this modification is potentially important for PK function during torpor. An analysis of the level of acetylation of muscle PK through Western blotting indicated that torpid PK was significantly more acetylated than the euthermic form of the enzyme (Fig. 3.6). Acetylation is emerging as a crucial and varied regulatory mechanism that is being found on an array of proteins within the cell. Recently, Zhao and colleagues (2010) found that many enzymes of glycolysis (including 
PK), gluconeogenesis, TCA cycle, urea cycle, fatty acid metabolism and glycogen metabolism were found to be acetylated in human liver tissue. Furthermore, studies on Salmonella enterica have shown that $\sim 90 \%$ of the enzymes of central metabolism were found to be acetylated and that the acetylation level varied depending the carbon source they were given; indicating that regulation is occurring through reversible acetylation (Wang et al., 2010). The effect of protein acetylation is varied, and studies have shown acetylation both positively and negatively affecting protein-protein interactions (Walter and Bienz, 1998; Dhalluin et al., 1999), and protein stability or half-life (Caron et al., 2005; Jeong et al., 2002). Few studies have been conducted to reveal the potential function of PK acetylation, but one study shows that acetylation of the M2 isoform of PK, targets this protein for lysosome-dependent degradation (Lv et al., 2011). While possible for squirrel skeletal muscle PK, it may be unlikely that acetylation targets this enzyme for degradation as it is a crucial component of glycolysis that is necessary during both torpor and interbout arousal. During hibernation the ground squirrel largely switches to using lipid oxidation for their primary fuel source, as evidenced by a respiratory quotient of $\sim 0.7$ (cited in Buck and Barnes, 2000). However, during the many periodic arousals that occur during hibernation the respiratory quotient quickly ramps up to $\sim 0.8$-1 indicating a rapid switch back to carbohydrate oxidation (Carey et al., 2003). It would be uneconomical with the ground squirrel's fixed fuel reserves to degrade PK during torpor only to have to synthesize it again upon arousal. Acetylation could potentially regulate protein-protein interactions between PK and another cellular proteins, or it could result in some of the kinetic or physical changes that were seen between euthermic and torpid PK. Clearly, there are many possible functional outcomes to PK 
acetylation and the role of ground squirrel muscle PK acetylation remains unknown and requires further investigation.

Purified skeletal muscle PK from Richardson's ground squirrels showed distinctly different kinetic properties between euthermic and torpid conditions, with torpid PK appearing to be less active at a low temperature that was typical of $\mathrm{T}_{\mathrm{b}}$ during dormancy as compared to euthermic PK. However, PK from the euthermic condition was well suited to function at normal mammalian $\mathrm{T}_{\mathrm{b}}$. ProQ Diamond phosphoprotein staining, Western blot analyses, and incubations that stimulated alkaline phosphatase indicated that at least some of the kinetic changes observed in this study were due to reversible phosphorylation. In addition to phosphorylation, skeletal muscle PK was found to be differentially acetylated with torpid PK showing greater levels of acetylation. The role of PK acetylation is unknown but may represent an important covalent modification during torpor. 
Table 3.1: Purification scheme for skeletal muscle PK from euthermic ground squirrels, U. richardsonii.

\begin{tabular}{llllll}
$\begin{array}{l}\text { Purification } \\
\text { Step }\end{array}$ & $\begin{array}{l}\text { Total } \\
\text { Protein } \\
(\mathrm{mg})\end{array}$ & $\begin{array}{l}\text { Total } \\
\text { Activity } \\
(\mathrm{U})\end{array}$ & $\begin{array}{l}\text { Specific } \\
\text { Activity } \\
(\mathrm{U} / \mathrm{mg})\end{array}$ & $\begin{array}{l}\text { Fold } \\
\text { Purification }\end{array}$ & \% Yield \\
\hline Supernatant & 19 & 19 & 0.99 & - & - \\
CM-Sephadex & 2.9 & 11 & 3.7 & 3.8 & 58 \\
Blue-Agarose & 0.010 & 4 & 390 & 390 & 21
\end{tabular}


Table 3.2: Kinetic properties of purified skeletal muscle PK from euthermic and torpid U. richardsonii

\begin{tabular}{llll|lll} 
& \multicolumn{3}{c|}{ Euthermic } & \multicolumn{3}{c}{ Torpid } \\
& $5^{\circ} \mathrm{C}$ & $22^{\circ} \mathrm{C}$ & $35^{\circ} \mathrm{C}$ & $5{ }^{\circ} \mathrm{C}$ & $22^{\circ} \mathrm{C}$ & $35^{\circ} \mathrm{C}$ \\
\hline $\mathrm{K}_{\mathrm{m}} \mathrm{PEP}(\mu \mathrm{M})$ & $25 \pm 2$ & $48 \pm 4$ & $33 \pm 5$ & $63 \pm 8^{*}$ & $65 \pm 4^{*}$ & $80 \pm 1^{*}$ \\
$\mathrm{~K}_{\mathrm{m}} \mathrm{ADP}(\mathrm{mM})$ & $0.32 \pm 0.05$ & $0.57 \pm 0.06$ & $0.44 \pm 0.04$ & $0.38 \pm 0.04$ & $0.22 \pm 0.01^{*}$ & $0.20 \pm 0.04^{*}$ \\
$\mathrm{~V}_{\max }(\mathrm{U} / \mathrm{mg})$ & $3.87 \pm 0.04$ & $14.3 \pm 0.5$ & $90 \pm 4$ & $5.6 \pm 0.3^{*}$ & $8.0 \pm 0.3^{*}$ & $47 \pm 3^{*}$
\end{tabular}

Data are means \pm SEM, $\mathrm{n}=4$ individual determinations on separate enzyme samples. $\mathrm{K}_{\mathrm{m}}$ values were determined at optimal cosubstrate concentrations (defined in Materials and Methods). * - Significantly different from the corresponding euthermic value using a two-tailed Student's t-test, $\mathrm{P}<0.05$. 
Table 3.3. Effects of various metabolites on purified euthermic and torpid U. richardsonii skeletal muscle PK.

\begin{tabular}{llll|lll} 
& \multicolumn{2}{c}{ Euthermic } & & & & \\
& & & & \\
& $5^{\circ} \mathrm{C}$ & $\mathrm{RT}$ & $35^{\circ} \mathrm{C}$ & $5^{\circ} \mathrm{C}$ & $\mathrm{RT}$ & $35^{\circ} \mathrm{C}$ \\
\hline $\mathrm{I}_{50} \mathrm{ATP}(\mathrm{mM})$ & $1.02 \pm 0.08$ & $3.0 \pm 0.3$ & $3.6 \pm 0.6$ & $5.6 \pm 0.4^{*}$ & $4.6 \pm 0.5$ & $6.6 \pm 0.5^{*}$ \\
(Fold Inactivation) & $(10 \pm 1)$ & $(4.6 \pm 0.8)$ & $(5.0 \pm 0.5)$ & $\left(3.0 \pm 0.3^{*}\right)$ & $(3.2 \pm 0.3)$ & $\left(2.4 \pm 0.2^{*}\right)$ \\
$\mathrm{K}_{\mathrm{a}} \mathrm{MgCl}_{2}(\mathrm{mM})$ & $0.7 \pm 0.1$ & $3.1 \pm 0.1$ & $0.9 \pm 0.1$ & $4 \pm 1$ & $1.2 \pm 0.3^{*}$ & $1.1 \pm 0.2$ \\
$($ Fold Activation) & $(7.4 \pm 0.8)$ & $(1.46 \pm 0.05)$ & $(2.1 \pm 0.2)$ & $(1.4 \pm 0.1)$ & $(1.38 \pm 0.05)$ & $(1.8 \pm 0.1)$ \\
$\mathrm{K}_{\mathrm{a}} \mathrm{KCl}(\mathrm{mM})$ & $3.9 \pm 0.6$ & $9 \pm 1$ & $2.7 \pm 0.6$ & $1.6 \pm 0.2^{*}$ & $9 \pm 1$ & $1.4 \pm 0.2$ \\
(Fold Activation) & $(1.7 \pm 0.1)$ & $(2.4 \pm 0.3)$ & $(1.8 \pm 0.2)$ & $(2.2 \pm 0.2)$ & $(1.8 \pm 0.1)$ & $(2.7 \pm 0.7)$
\end{tabular}

Data are means \pm SEM, $\mathrm{n}=4$ individual determinations on separate enzyme samples. $\mathrm{K}_{\mathrm{a}}$ and $\mathrm{I}_{50}$ values were determined at suboptimal cosubstrate concentrations (defined in Materials and Methods). Note that alanine, aspartate, citrate and fructose-1,6bisphosphate were also tested as effectors but did not affect PK from either euthermic or torpid animals at any of the temperatures tested. * - Significantly different from the corresponding euthermic value using a two-tailed Student's t-test, $\mathrm{P}<0.05$. 
Table 3.4. The effect of chemical denaturants, urea and guanidine hydrochloride $(\mathrm{GnHCl})$, on the activity of purified euthermic and torpid S. richardsonii skeletal muscle PK.

\begin{tabular}{|c|c|c|c|c|c|c|}
\hline & \multicolumn{3}{|l|}{$\mathrm{I}_{50}$ Urea (M) } & \multicolumn{3}{|l|}{$\mathrm{I}_{50} \mathrm{GnHCl}(\mathrm{M})$} \\
\hline & $5^{\circ} \mathrm{C}$ & RT & $35^{\circ} \mathrm{C}$ & $5^{\circ} \mathrm{C}$ & RT & $35^{\circ} \mathrm{C}$ \\
\hline Euthermic & $0.90 \pm 0.02^{b}$ & $1.26 \pm 0.06$ & na & $0.38 \pm 0.005^{b}$ & $0.44 \pm 0.005$ & na \\
\hline Torpid & $1.73 \pm 0.01^{\mathrm{ab}}$ & $1.19 \pm 0.03^{b}$ & $0.60 \pm 0.03^{\mathrm{a}}$ & $0.35 \pm 0.005^{\mathrm{ab}}$ & $0.55 \pm 0.03^{\mathrm{ab}}$ & $0.13 \pm 0.01^{\mathrm{a}}$ \\
\hline
\end{tabular}

Data are means \pm SEM, $\mathrm{n}=4$ individual determinations on separate enzyme samples. The temperature values reported here are the incubation temperatures, whereas all assays were performed at RT. $\mathrm{I}_{50}$ values were determined under $\mathrm{V}_{\text {max }}$ conditions. ${ }^{\mathrm{a}}$ - Significantly different from the corresponding euthermic value using a two-tailed Student's t-test, $\mathrm{P}<0.05$. ${ }^{\mathrm{b}}$ - Significantly different from the value at RT for euthermic PK or the value at $35^{\circ} \mathrm{C}$ for torpid PK. 


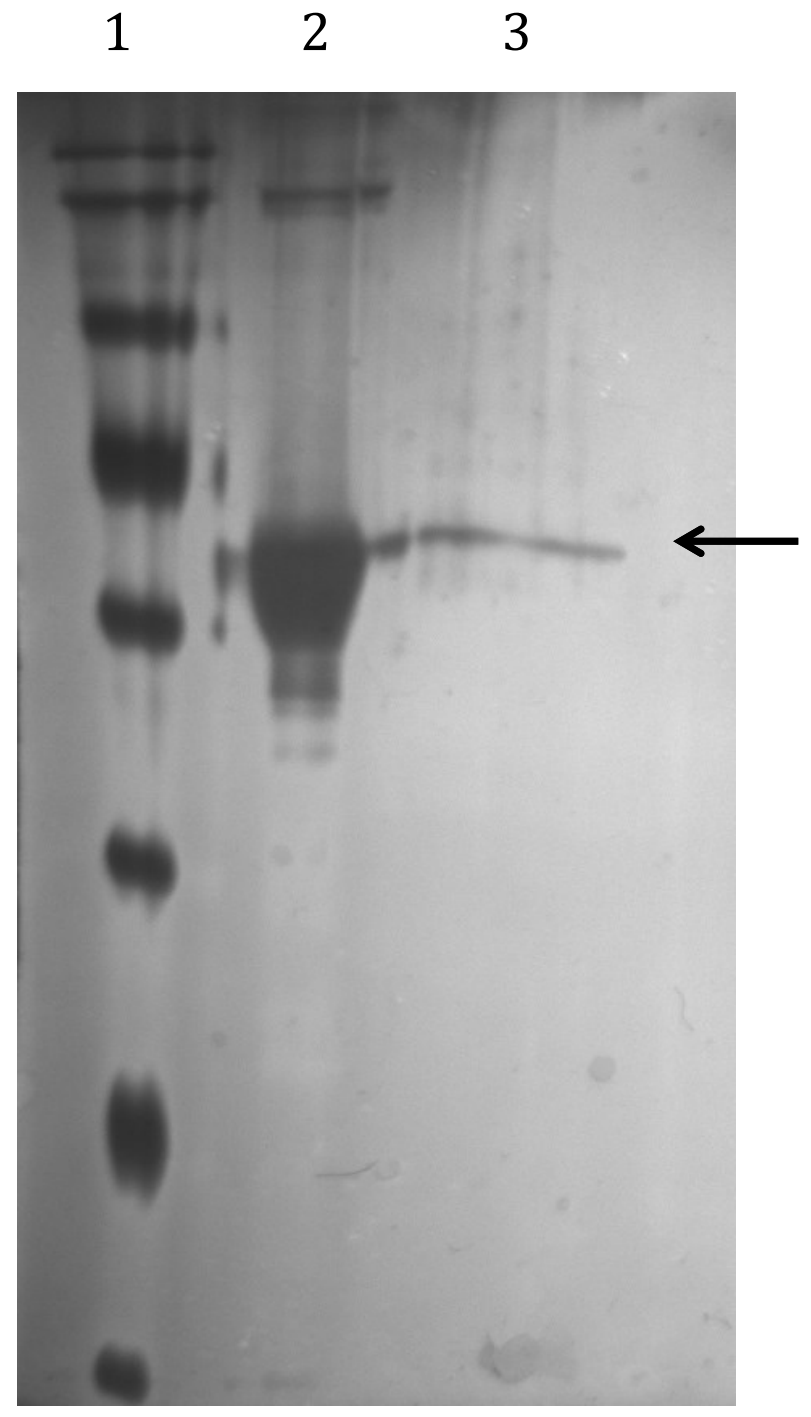

Figure 3.1 Purified euthermic PK from the skeletal muscle of U. richardsonii. Lane 1 shows the protein ladder; Lane 2 shows the protein eluted from a $\mathrm{CM}^{-}$Sephadex column; Lane 3 shows the purified PK sample after the blue-agarose column. The arrow indicates the location of PK subunits between the $55 \mathrm{kDa}$ and the $72 \mathrm{kDa}$ molecular weight standards in lane 1. 


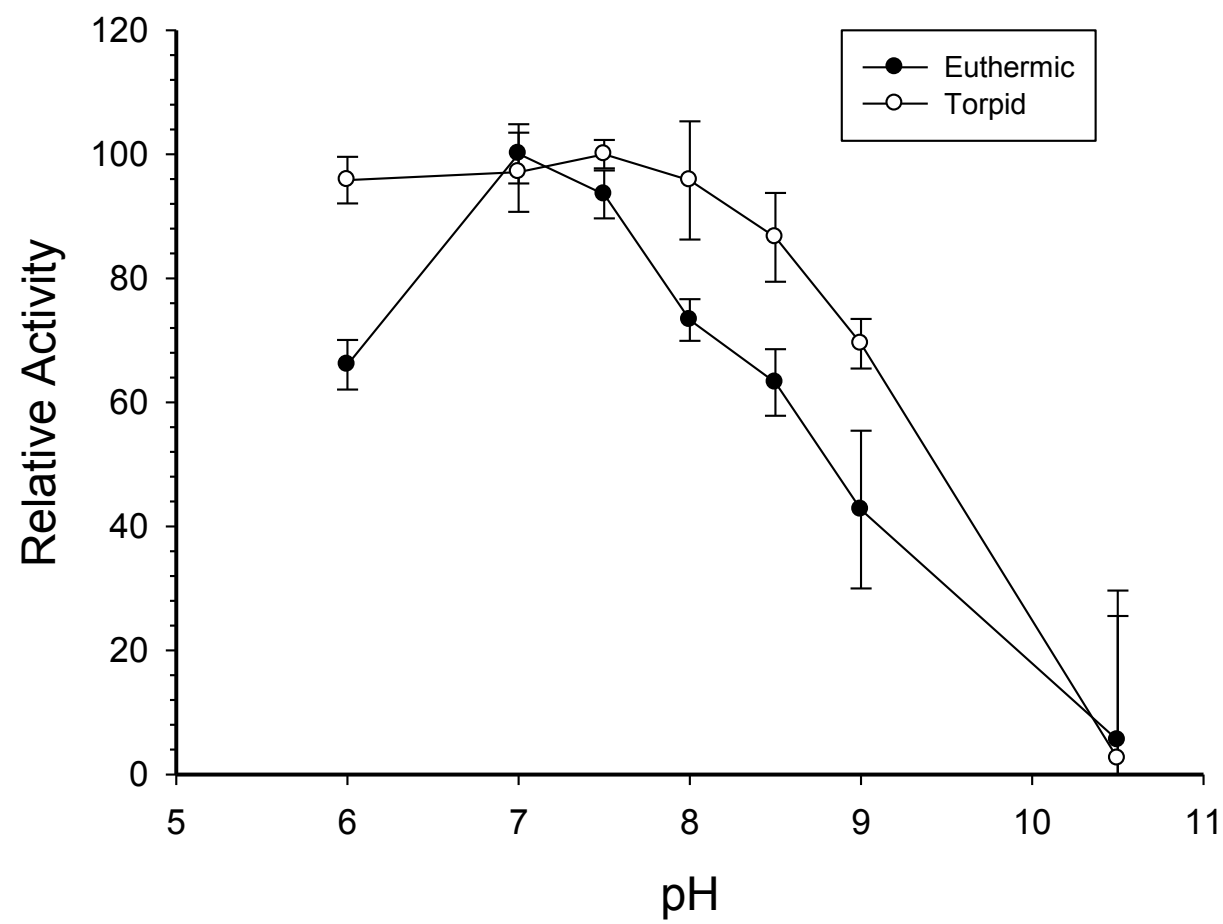

Figure 3.2 $\mathrm{pH}$ curve for euthermic and torpid PK from the skeletal muscle of $U$.

richardsonii. The data are means $\pm \mathrm{SEM}, \mathrm{n}=3$ separate determinations on independent samples of purified enzyme. 


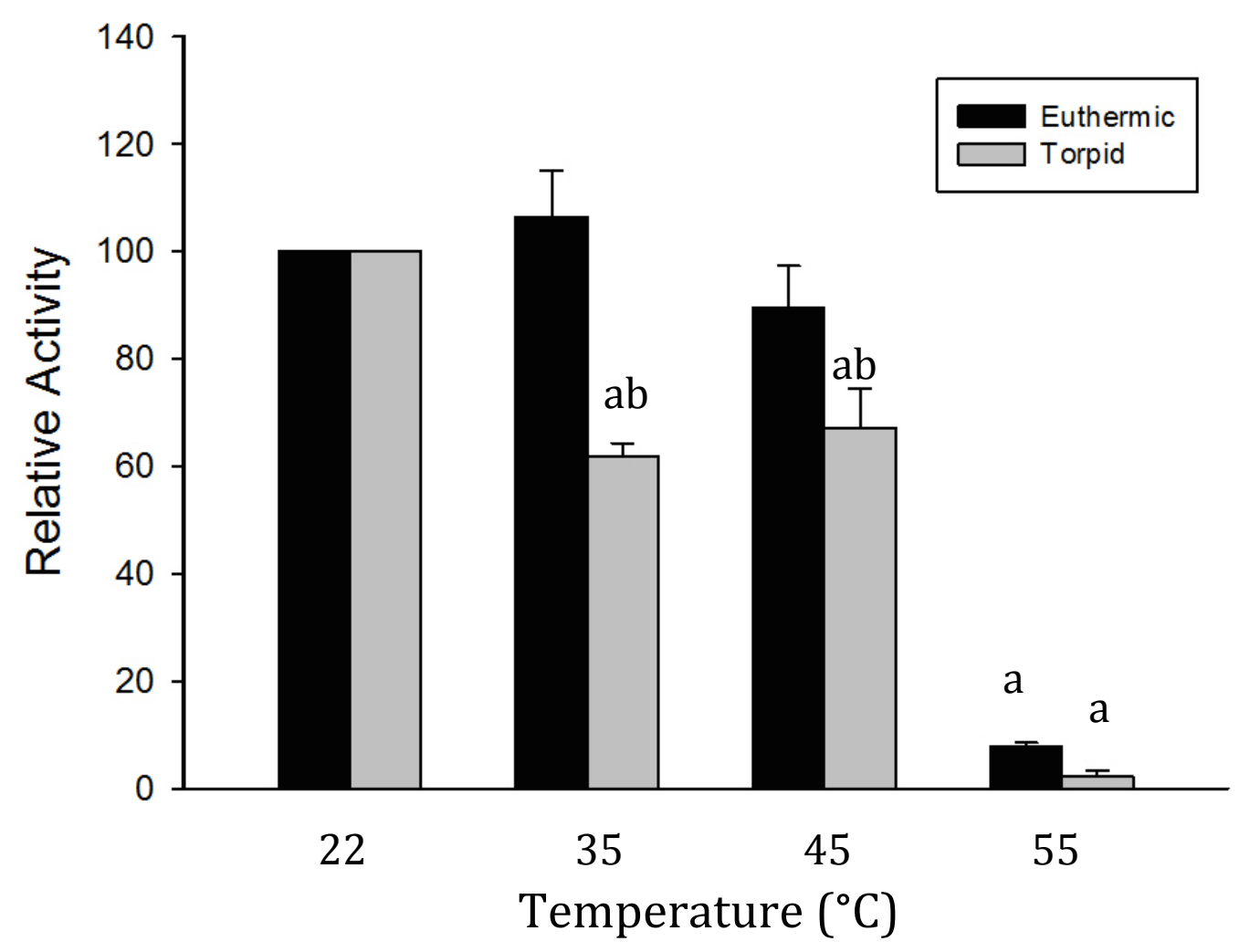

Figure 3.3 Thermal stability of euthermic and torpid PK from the skeletal muscle of $U$. richardsonii. Data are means $\pm \mathrm{SEM}, \mathrm{n}=4$ independent determinations on separate enzyme samples. ${ }^{\text {a }}$ - Significantly different from the corresponding value for the same condition (euthermic or torpid) at room temperature $\left(22^{\circ} \mathrm{C}\right) ;{ }^{\mathrm{b}}-$ significantly different from the euthermic value at the same temperature. Significant differences were determined by the Holm-Sidak method, $\mathrm{p}<0.05$. 


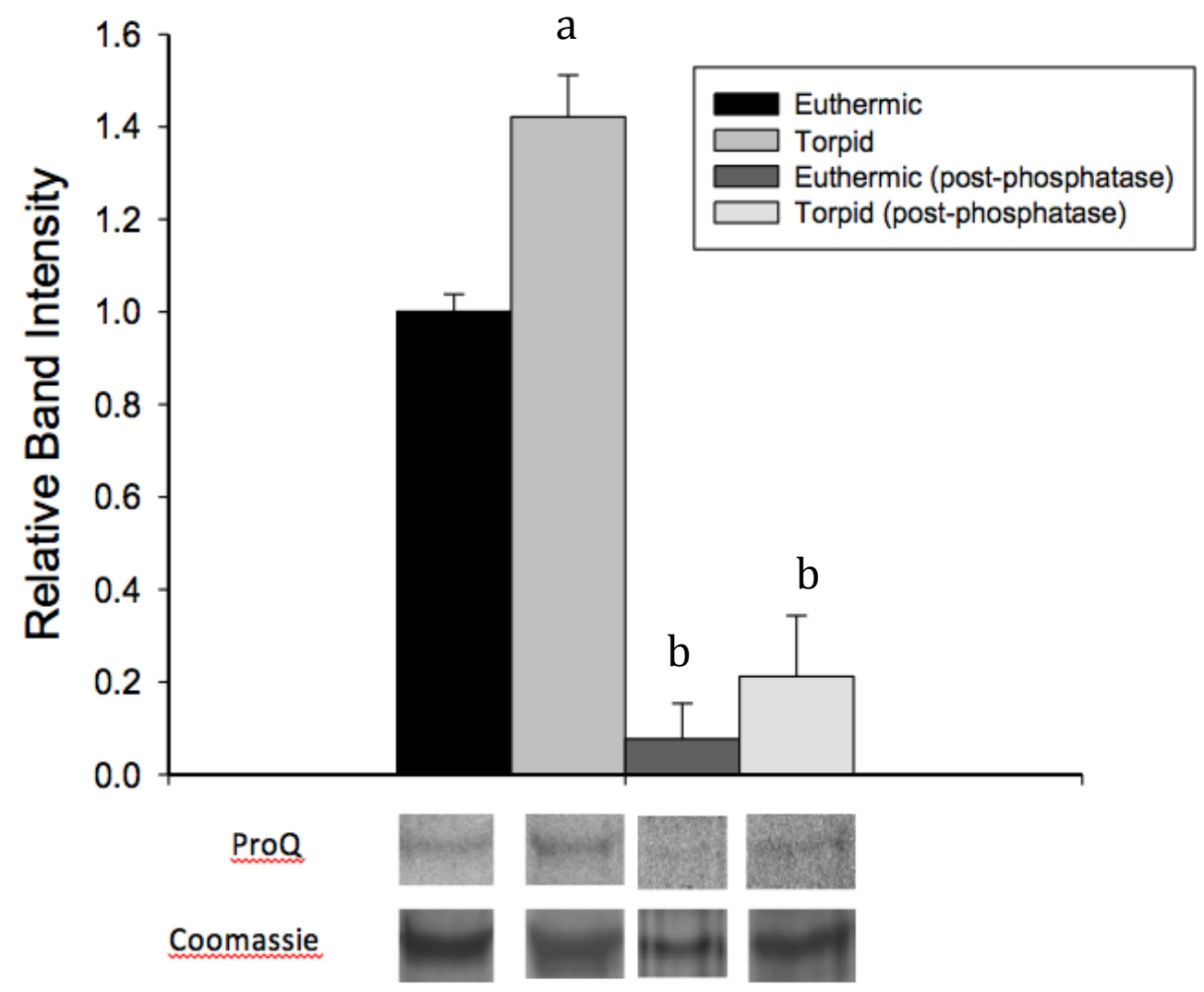

Figure 3.4 Phosphorylation state of euthermic and torpid PK from the skeletal muscle of U. richardsonii. Histogram bars that are indicated as 'post-phosphatase' are for purified PK samples that were incubated with alkaline phosphatase (see Materials and Methods for details) prior to electrophoresis and staining with ProQ Diamond. Data are means \pm SEM, $\mathrm{n}=4$ determinations on independent enzyme samples. ${ }^{\mathrm{a}}-$ Significantly different from the euthermic relative band intensity without any phosphatase treatment. ${ }^{\mathrm{b}}-$ Significantly different from the corresponding value without phosphatase treatment. The statistical test used for these determinations was the Holm-Sidak method, $\mathrm{p}<0.05$. 


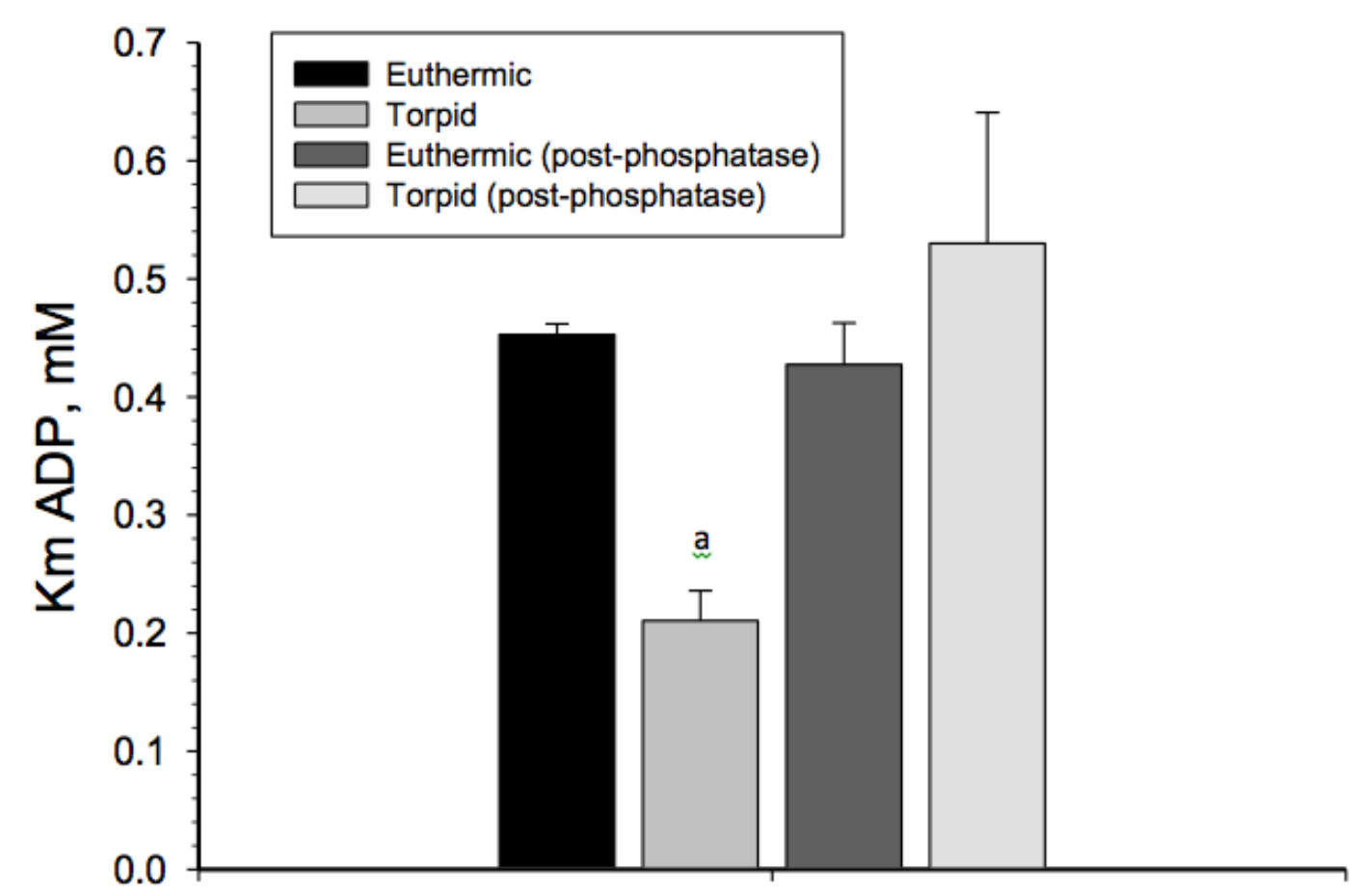

Figure 3.5 Effect of alkaline phosphatase incubations on the $K_{m}$ ADP of PK. $K_{m}$ values are means \pm SEM, $\mathrm{n}=4$ independent determinations on different incubated samples. ${ }^{\mathrm{a}}$ Significantly different from the corresponding euthermic PK value using the Holm-Sidak method, $\mathrm{p}<0.05$. 


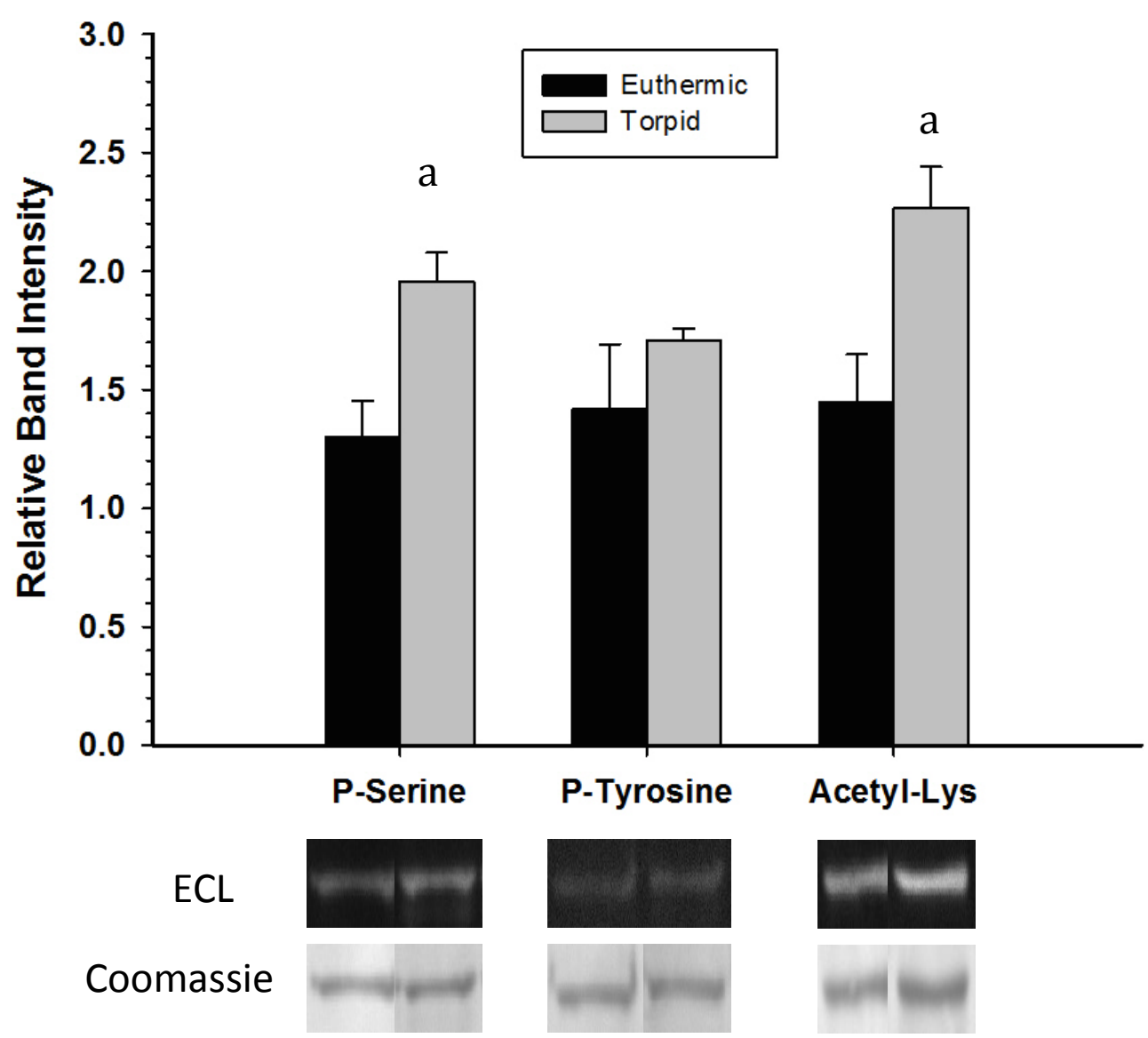

Figure 3.6 Analysis of posttranslational modification to skeletal muscle PK from $U$. richardsonii as assessed through Western blots. Relative band intensities are means \pm SEM, $\mathrm{n}=4$ determinations on independently purified PK samples. a - Significantly different from the corresponding euthermic value using Student's t-test, $\mathrm{p}<0.05$. 


\section{Chapter 4}

\section{Regulation of skeletal muscle fructose-1,6- bisphosphatase from the hibernating ground squirrel, Ictidomys tridecemlineatus}




\section{Introduction}

One of the key features of mammalian hibernation is an alteration in fuel metabolism that is dependent on the animal's stage within the hibernation cycle. For instance, during torpor, it is well established that most tissues switch to non-carbohydrate fuels and suppress carbohydrate catabolism (cited in Buck and Barnes, 2000; Tashima et al., 1970), while during interbout arousal carbohydrate catabolism becomes significant once again (Lyman and Leduc, 1953). The state of gluconeogenesis during hibernation is somewhat less clear, especially with respect to mammalian muscle tissue. Several studies suggest that gluconeogenesis decreases during torpor as evidenced by decreases in skeletal muscle glycogen levels (Zimny and Tyrone, 1957; Galster and Morrison, 1970; Galster and Morrison, 1975). Other studies have indicated that skeletal muscle glycogen increases or is maintained during torpor, which is suggestive of increased gluconeogenesis (Hannon and Vaughn, 1961; el Hachimi et al., 1990; James et al., 2013). While many of these studies have focused on muscle carbohydrate stores as an indicator of gluconeogenesis, no study has taken an in-depth look at some of the key gluconeogenic enzymes that mediate this process.

D-Fructose-1,6-bisphosphatse (FBPase; EC 3.1.3.11) is a critical member of the gluconeogenic pathway and catalyzes the reaction that is counter to phosphofructokinase. Specifically, FBPase catalyzes the removal of a phosphate from D-fructose-1,6bisphosphate to form fructose-6-phosphate, in the presence of divalent cations, typically magnesium or manganese (reaction illustrated in Appendix IG). Regulation of this enzyme may be accomplished by intracellular AMP (Underwood and Newsholme, 1965), fructose-2,6-bisphosphate (F26P2; Pilkis et al., 1981), as well as by reversible protein 
phosphorylation (Ekdahl, 1992; Rakus et al., 2003). This intricate regulatory network and its importance to the gluconeogenic pathway makes FBPase an intriguing enzyme to study in an animal that exhibits significant flux in carbohydrate metabolism - as is seen in the 13-lined ground squirrel during hibernation. The present investigation aims to determine the physical, kinetic and regulatory properties of skeletal muscle FBPase in an attempt to determine its role in mediating gluconeogenesis during ground squirrel hibernation.

\section{Materials and Methods}

Animals

Animal collection and tissue extraction is as described in Chapter 2.

Tissue Preparation and Purification of FBPase

Frozen skeletal muscle tissue was homogenized 1:5 w/v in homogenization buffer (12.5 mM 2-(N-morpholino)ethanesulfonic acid (MES), pH 6.5, $25 \mathrm{mM} \mathrm{NaF}, 1.25 \mathrm{mM}$ EDTA, $1.25 \mathrm{mM}$ EGTA, $5 \mathrm{mM} \beta$-mercaptoethanol, and 5\% glycerol) using a Polytron homogenizer, with a few crystals of phenylmethylsulphonyl fluoride added prior to homogenization. The resulting crude preparation was centrifuged at 13, $500 \mathrm{~g}$ for $30 \mathrm{~min}$ at $5^{\circ} \mathrm{C}$ in an Eppendorf centrifuge (5810 R). Supernatant was then decanted and held at $4^{\circ} \mathrm{C}$ until use.

Muscle FBPase was partially purified by ion exchange chromatography. Specifically, carboxymethyl- (CM-)Sephadex beads were placed into a column to the approximate dimensions of $4 \times 1 \mathrm{~cm}$ (height $\mathrm{x}$ diameter), and equilibrated in homogenization buffer. Following equilibration, $1.5 \mathrm{~mL}$ of crude extract was layered on top of the column and allowed to settle into the beads for several minutes. The beads 
were then washed with $30 \mathrm{~mL}$ of homogenization buffer, which was sufficient to reduce the absorbance at $280 \mathrm{~nm}$ of the collected wash fraction to that of just buffer. The enzyme was then eluted with a linear salt gradient of $0-1 \mathrm{M} \mathrm{KCl}$ made in homogenization buffer. The top three activity fractions were pooled and used in FBPase assays.

FBPase Assay

Partially purified 13-lined ground squirrel skeletal muscle FBPase was assayed spectrophotometrically using a Thermo Labsystems Multiskan spectrophotometer at 340 $\mathrm{nm}$. Optimal assay conditions were $50 \mathrm{mM}$ imidizole, $\mathrm{pH}$ 7.5, $0.1 \mathrm{mM}$ D-fructose-1,6bisphosphate $\left(\mathrm{F} 1,6 \mathrm{P}_{2}\right), 0.25 \mathrm{mM} \mathrm{NADP}^{+}, 7.5 \mathrm{mM} \mathrm{MgSO}_{4}, 0.5 \mathrm{U}$ of phosphoglucoisomerase (Sigma), and $0.5 \mathrm{U}$ of glucose-6-phosphate dehydrogenase (Sigma). Reactions were started with the addition of $40 \mu \mathrm{L}$ of partially purified extract. The sub-optimal assay conditions used to determine $\mathrm{I}_{50}$ values were $50 \mathrm{mM}$ imidizole, $\mathrm{pH}$

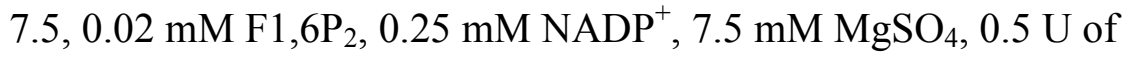
phosphoglucoisomerase, and $0.5 \mathrm{U}$ of glucose-6-phosphate dehydrogenase. Coupling enzymes were always added in excess. This was ensured by the measurement of FBPase reaction rates after doubling the amount of enzyme added to the reaction wells. The reaction rate doubled as expected and thus the coupling agents were said to be in excess. Assays were again started with the addition of $40 \mu \mathrm{L}$ of partially purified extract. This amount of enzyme produced linear Abs. vs. time curves and ensured the measurement of initial reaction rates. Assays were performed at room temperature $\left(22^{\circ} \mathrm{C}\right)$. Enzyme extracts remained stable for approximately 3 days without reduction in activity (data not shown). All experiments were performed within this time period. 
Deinhibition studies were conducted by assaying FBPase under sub-optimal conditions (outlined above) in the presence of $2 \mu \mathrm{M}$ AMP or fructose-2,6-bisphosphate $\left(\mathrm{F} 2,6 \mathrm{P}_{2}\right)\left(\right.$ concentrations that were near experimentally determined $\mathrm{I}_{50}$ values) as well as $10 \mathrm{mM}$ Mg:citrate or histidine.

Data was analyzed and protein content determined as described in Chapter 2.

\section{Urea Denaturation of FBPase}

Urea-induced unfolding of skeletal muscle FBPase was conducted as outlined in Chapter 2. The only difference was that the concentration of urea ranged from $0-4.5 \mathrm{M}$. SDS Polyacrylamide Gel Electrophoresis, Immunoblotting, and ProQ Diamond Phosphoprotein Staining

Partially purified muscle FBPase from both ACR and LH conditions were mixed 2:1 v:v with SDS loading buffer and boiled for 5 minutes, cooled on ice and frozen at $-20^{\circ} \mathrm{C}$ until use. SDS resolving gels $(10 \%)$ were run as directed in Chapter 2. Moreover, ProQ Diamond phosphoprotein staining and immunoblotting was performed as described in Chapter 2. An important difference was the use of commercially pure rabbit muscle FBPase (Boehringer Mannheim) that was also loaded onto the gel to confirm the correct location of the FBPase subunits.

\section{Phospho-dephospho Incubations}

To assess whether FBPase kinetic parameters were regulated by reversible phosphorylation, incubations were conducted that stimulated endogenous protein kinases as well as an exogenous protein phosphatase, and the key kinetic parameter that changed between ACR and LH conditions was reassessed (i.e. $\mathrm{K}_{\mathrm{m}} \mathrm{F} 1,6 \mathrm{P}_{2}$ ). 
Samples were prepared as described above and subsequently centrifuged through a G50 Sephadex column, pre-equilibrated in incubation buffer (25 mM MES, pH 6.5, 5\% v:v glycerol, and $5 \mathrm{mM} \beta$-mercaptoethanol), at $2500 \mathrm{RPM}$ for $1 \mathrm{~min}$. The resulting eluent was then mixed 1:1 v:v with incubation buffer plus the following additions:

1) STOP (inhibition of kinases and phosphatases) - $1.25 \mathrm{mM}$ EDTA, $1.25 \mathrm{mM}$ EGTA, $12.5 \mathrm{mM} \mathrm{NaF}$.

2) Total Kinase - $2 \mathrm{mM}$ cAMP, $10 \mathrm{mM}$ ATP, $20 \mathrm{mM} \mathrm{MgCl} 2,25 \mathrm{mM} \mathrm{NaF}, 10 \mathrm{mM}$ $\mathrm{Na}_{3} \mathrm{VO}_{4}, 2 \mathrm{mM}$ cGMP, $2.5 \mathrm{mM} \mathrm{CaCl} 2,14 \mu \mathrm{g} / \mathrm{mL}$ phorbol 12-myristate 13-acetate (PMA), $1 \mathrm{mM}$ AMP, and $1 \mathrm{U}$ of calmodulin activity.

3) Acid Phosphatase - $20 \mathrm{mM} \mathrm{MgCl} 2,10 \mathrm{mM}$ EDTA, and $1 \mathrm{U}$ of acid phosphatase activity (Sigma).

Samples were incubated for $3 \mathrm{~h}$ at $4^{\circ} \mathrm{C}$ with occasional vortexing. Following this incubation each sample was partially purified as previously described. Immediately following partial purification of muscle FBPase, the $\mathrm{K}_{\mathrm{m}} \mathrm{F} 1,6 \mathrm{P}_{2}$ was reassessed.

\section{Results}

\section{FBPase Partial Purification and Kinetics}

Skeletal muscle FBPase was purified 22-fold using a $\mathrm{CM}^{-}$Sephadex ion exchange column with an overall yield of $155 \%$ (Figure 4.1; Table 4.1). This enzyme preparation was subsequently used to assess FBPase kinetic properties, and in doing so, several significant differences were identified between the behaviour of this enzyme when it originated from ACR or LH conditions. For instance, $\mathrm{K}_{\mathrm{m}} \mathrm{F} 1,6 \mathrm{P}_{2}$ was nearly 2-fold higher for FBPase from the torpid condition as compared to the control condition, with a concomitant 2-fold increase in the Hill coefficient (indicating greater sigmoidicity of the 
$\mathrm{V}$ vs [S] relationship) for the enzyme from the torpid state (Table 4.2 and Fig. 4.3).

Furthermore, $\mathrm{I}_{50}$ values for two well-known inhibitors of FBPase, $\mathrm{F} 2,6 \mathrm{P}_{2}$ and AMP, were both approximately 2-fold higher for FBPase from the LH condition as compared to FBPase from ACR muscle (Table 4.2). The effect of AMP, but not F2,6P2, was found to be negated by the addition of $10 \mathrm{mM} \mathrm{Mg}$ :citrate or $10 \mathrm{mM}$ histidine (Fig. 4.3).

Muscle FBPase was also greatly affected by the addition of a common denaturant, urea. FBPase from the LH condition displayed a 3-fold higher $\mathrm{I}_{50}$ urea as compared to that of the control-derived enzyme (Fig. 4.4). Specifically, the $I_{50}$ urea for FBPase from the $\mathrm{LH}$ condition was $3.7 \pm 0.3 \mathrm{M}$ while FBPase from the control condition was only 1.2 $\pm 0.2 \mathrm{M}$.

\section{FBPase Posttranslational Modification}

Partially purified muscle FBPase was subjected to SDS-PAGE and then to ProQ Diamond phosphoprotein staining and Western blot analysis with antibodies detecting phospho-serine, -threonine, or -tyrosine residues. ProQ Diamond phosphoprotein staining of muscle FBPase resulted in near identical levels of phosphorylation being detected between ACR and LH conditions (Fig. 4.5). The same was true of phosphothreonine and phospho-tyrosine Western blots, with no significant differences between FBPase from the ACR of LH conditions being detected. There was however, a near $50 \%$ increase in phospho-serine levels detected on FBPase from the torpid condition as compared to the control condition (Fig. 4.5).

The potential effects of increased phospho-serine detection during torpor was investigated using incubations that stimulated endogenous protein kinases or a commercial acid phosphatase and subsequent re-examination of $\mathrm{K}_{\mathrm{m}} \mathrm{F} 1,6 \mathrm{P}_{2}$. Following 
incubations that stimulated a number of protein kinases within the cell, $\mathrm{K}_{\mathrm{m}} \mathrm{F} 1,6 \mathrm{P}_{2}$ for ACR FBPase increased significantly to values typically seen during torpor (Fig. 4.7). In response to the same incubation conditions, $\mathrm{K}_{\mathrm{m}} \mathrm{F} 1,6 \mathrm{P}_{2}$ for LH FBPase did not change. Conversely, when skeletal muscle FBPase was incubated with commercial acid phosphatase there was a significant reduction in $\mathrm{K}_{\mathrm{m}} \mathrm{F} 1,6 \mathrm{P}_{2}$ for FBPase from the torpid animals, while the value for FBPase from the euthermic animals did not change (Fig. 4.7).

Posttranslational acetylation of FPBase was also investigated using Western blot analysis of partially purified muscle samples. These blots indicated that FBPase from the torpid condition was approximately 3 -fold more acetylated than FBPase from the control state (Fig. 4.6).

\section{Discussion}

Metabolic reorganization during ground squirrel torpor requires coordinated regulation of key metabolic enzymes, including those of glycolysis and gluconeogenesis. While much work has been completed with respect to the regulation of glycolytic output, few studies have investigated gluconeogenic output during torpor, especially within mammalian skeletal muscle. Those studies that have analyzed skeletal muscle gluconeogenesis have arrived at somewhat contradictory conclusions with some indicating an increase in gluconeogenesis during torpor (Hannon and Vaughn, 1961; el Hachimi et al., 1990; James et al., 2013) and others indicating significant decreases (Zimny and Tyrone, 1957; Galster and Morrison, 1970; Galster and Morrison, 1975). None of these studies have evaluated the responses of the actual enzymes involved in regulating gluconeogenesis to determine if there is enzymatic evidence to support an 
altered capacity for gluconeogenesis during hibernation. The current study investigates the regulatory properties of skeletal muscle FBPase originating from euthermic and torpid animals in an attempt shed light on the state of gluconeogenesis during torpor.

Partially purified ground squirrel muscle FBPase displayed numerous kinetic changes between ACR and LH conditions. Hibernation had a distinct effect on the substrate affinity of the enzyme with a nearly 2-fold increase in $\mathrm{K}_{\mathrm{m}} \mathrm{F}$ 1,6 $\mathrm{P}_{2}$ (from 21 to 40 $\mu \mathrm{M}$ ) for FBPase derived from dormant animals as compared to that from euthermic animals (Table 4.2). This increase may be significant in vivo since the concentration of $\mathrm{F} 1,6 \mathrm{P}_{2}$ typically found in muscle is in the low micromolar range, and thus the reduced substrate affinity of FBPase during torpor could substantially affect enzyme rates in vivo. However, Storey and Kelly (1994) reported that the concentration of F1,6 $\mathrm{P}_{2}$ in the skeletal muscle of the jumping mouse, Zapus hudsonius, was approximately $20 \mu \mathrm{M}$ during euthermia and nearly $40 \mu \mathrm{M}$ during torpor. If the same is true for 13-lined ground squirrel $\mathrm{F} 1,6 \mathrm{P}_{2}$ concentration in muscle, then FBPase from the torpid animal may continue to function at about half of its maximal velocity, as in euthermia. However, $\mathrm{V}_{\max }$ measurements for muscle FBPase from the two conditions do indicate that FBPase from the LH condition is significantly less active than ACR muscle FBPase, and similar

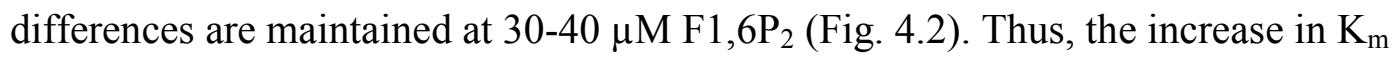
$\mathrm{F} 1,6 \mathrm{P}_{2}$ and decrease in FBPase $\mathrm{V}_{\max }$ may signal a suppression of FBPase functioning during torpor.

In apparent contradiction to the suppression of muscle FBPase activity is the significant increases in $\mathrm{I}_{50} \mathrm{AMP}$ and $\mathrm{I}_{50} \mathrm{~F} 2,6 \mathrm{P}_{2}$ for FBPase from the LH condition as compared to control (Table 4.2). The $\mathrm{I}_{50}$ values are well below the expected 
concentrations for AMP and F2,6 $\mathrm{P}_{2}$ within mammalian muscle; $\mathrm{AMP}$ and $\mathrm{F} 2,6 \mathrm{P}_{2}$ being present at approximately $0.1 \mathrm{mM}$ and $0.04 \mathrm{mM}$, respectively (Minatogawa and Hue, 1984; Storey and Kelly, 1994; English and Storey, 2000). At these concentrations in vivo it would be expected that FBPase is strongly, if not completely, inhibited during both euthermia and torpor, however this is unlikely to be the case. It is more likely that some intracellular molecule(s) is/are capable of counteracting AMP and F2,6 $\mathrm{P}_{2}$-dependent inhibition, effectively deinhibiting FBPase. Deinhibition is a well-known phenomenon but one that is not exceedingly common. It is important to note that deinhibition is distinct from activation since the deinhibiting molecule does not activate the enzyme in question when added to the enzyme without the inhibitor. Deinhibition studies conducted here indicated that both $\mathrm{Mg}$ :citrate and histidine are capable of negating the inhibitory effects of AMP but not F2,6P 2 (Fig. 4.3). Intracellular citrate levels are expected to increase substantially during torpor since elevated citrate typically accompanies increased fatty acid oxidation, which is the major fuel during dormancy (Brooks and Storey, 1992). Free histidine is generally believed to be below $1 \mathrm{mM}$ in mammalian muscle tissue, and thus may play a minor role in preventing AMP-mediated inhibition of FBPase (Newsholme and Leech, 2010). The mechanism by which this deinhibition occurs is unknown. The fact that citrate and histidine deinhibited only the non-competitive inhibitor, AMP, suggests some association between FBPase allosteric sites and the deinhibiting compounds (Pontremoli and Horecker, 1971; Pilkis et al., 1981; Van Schaftingen and Hers, 1981). An alternative to other molecules, such as citrate or histidine, overcoming AMP- and/or F2,6 $\mathrm{P}_{2}$-induced inhibition within the cell is the idea that measured AMP and $\mathrm{F} 2,6 \mathrm{P}_{2}$ concentrations are not representative of the actual 
concentration of free molecules in vivo. This was demonstrated previously in rat livers where the measured concentration of each inhibitor was enough to completely inhibit FBPase, however, clear evidence existed that there was cycling occurring between glucose and triose phosphates (reviewed in Hue and Rider, 1987). This suggests that actual free AMP and $\mathrm{F} 2,6 \mathrm{P}_{2}$ is likely far lower than measured in vitro values, and also lower than the measured $\mathrm{I}_{50}$ values.

The kinetic differences identified above for muscle FBPase between euthermic and hibernating states suggest that there is some structural difference in this enzyme between the two conditions. Urea-induced unfolding is a common method in determining if two enzymes are structurally distinct, and in this study, FBPase from the torpid animal exhibited an approximately3-fold higher $\mathrm{I}_{50}$ urea than the euthermic enzyme (Fig. 4.3). This reduced sensitivity to urea argues that the enzyme from the hibernating animal is more structurally stable than the euthermic enzyme, but this is somewhat contradictory to the generally accepted theory that cold-adapted enzymes maintain activity at low temperatures by increased structural flexibility, which inherently makes the enzyme less stable (Huston et al., 2008). However, increased structural stability likely means decreased enzyme functionality, which supports the kinetic findings in this study that indicate FBPase is less active during torpor.

Structural and functional dissimilarities of the same enzyme from two different conditions is often caused by the posttranslational modification of that particular enzyme. One of the most common and crucial covalent modifications to enzymes is reversible phosphorylation. The phosphorylation states of the euthermic and hibernating forms of muscle FBPase were assessed by ProQ Diamond phosphoprotein staining of FBPase 
subunits separated on an SDS-gel, as well as through the use of phospho-specific antibodies. Figure 4.4 shows that staining with ProQ Diamond revealed that total phosphate bound to FBPase from control and torpid conditions was not significantly different. This finding coincided with the results of the Western blots detecting phosphothreonine, or -tyrosine residues, which also showed no change for FBPase between the two conditions (Fig. 4.5). Levels of phospho-serine, on the other hand, indicated that LH FBPase was significantly more phosphorylated than the euthermic form of the enzyme. Phosphorylation of FBPase is not uncommon and has been shown to occur in a variety of organisms. Indeed, FBPase has been shown to be phosphorylated in the freeze-avoiding gall moth, Epiblema scudderiana (Muise and Storey, 1997), yeast, Saccharomyces cerevisae (Rose et al., 1988), as well as in both mammalian liver and muscle (Ekdahl, 1988; Rakus et al., 2003), with a variety of effects, including changes in FBPase kinetics. In vitro incubations that stimulated endogenous protein kinases or acid phosphatase in this study indicated that, indeed, ground squirrel muscle FBPase phosphorylation was linked to changes in $\mathrm{K}_{\mathrm{m}} \mathrm{F} 1,6 \mathrm{P}_{2}$ between euthermic and torpid conditions; LH FBPase being in the high phosphate form and ACR FBPase being in the low phosphate form (Fig. 4.7). This link between phosphorylation and changes in $\mathrm{K}_{\mathrm{m}} \mathrm{F}$ 1,6 $\mathrm{P}_{2}$ have been observed previously (Rakus and Dzugaj, 2000; Rakus et al., 2003), which suggests that regulation of FBPase by reversible phosphorylation may be a fairly conserved mechanism.

Other forms of protein posttranslational mechanisms exist and these have recently come to the forefront as regulators of enzyme function. One of these is protein acetylation. Indeed, the present study shows that muscle FBPase was differentially acetylated between ACR and LH conditions. Specifically, skeletal muscle FBPase 
displayed a nearly 3 -fold higher level of acetylation during hibernation as compared to the ACR condition (Fig. 4.6). Acetylation of FBPase has been reported previously, however the effect of such a modification was unknown (Simon et al., 2012). Interestingly, acetylation of other gluconeogenic enzymes, such as phosphoenolpyruvate carboxykinase (PEPCK), has been found to mediate flux through the gluconeogenic pathway in mammalian cell lines (Jiang et al., 2011; Xiong et al., 2011). Specifically, acetylation of PEPCK stimulates the ubiquitination, destabilization and eventual degradation of PEPCK leading to decreased gluconeogenic flux. This is unlikely to be the case for ground squirrel FBPase due to increased FBPase stability determined in this study. However, the study of PEPCK acetylation, as well as several other studies, do suggest a key role of reversible protein acetylation in the regulation of gluconeogenesis as well as metabolism as a whole (Sadoul et al., 2010; Zhao et al., 2010). Further experimentation on FBPase acetylation should focus on its potential role in mediating FBPase stability and/or activity during ground squirrel torpor.

This study has shown that muscle FBPase is kinetically and physically distinct between euthermic and torpid conditions. FBPase from the torpid condition appears to be suppressed during torpor with an increase in protein rigidity that may augment the decrease in enzyme activity during torpor. The suppression of FBPase function during torpor appears to be linked to reversible phosphorylation, with increased phosphorylation being linked to decreased enzyme function during torpor. The torpor enzyme form also showed a substantial increase in protein acetylation, which may represent a novel regulatory mechanism for this enzyme during hypometabolism. 
Table 4.1: Partial purification of FBPase from 13-lined ground squirrel ACR muscle.

\begin{tabular}{llllll} 
Purification & $\begin{array}{l}\text { Total } \\
\text { Protein } \\
(\mathrm{mg})\end{array}$ & $\begin{array}{l}\text { Total } \\
\text { Activity } \\
(\mathrm{mU})\end{array}$ & $\begin{array}{l}\text { Specific } \\
\text { Activity } \\
(\mathrm{mU} / \mathrm{mg})\end{array}$ & $\begin{array}{l}\text { Fold } \\
\text { Purification }\end{array}$ & \%Yield \\
\hline Crude Extract & 14 & 9.8 & 0.7 & - & - \\
$\mathrm{CM}^{-}$Sephadex & 1 & 15.1 & 15.1 & 22 & 155
\end{tabular}


Table 4.2: Kinetic comparison of 13-lined ground squirrel skeletal muscle FBPase from ACR and LH conditions.

\begin{tabular}{lll} 
Kinetic Parameter & ACR & LH \\
\hline $\mathrm{K}_{\mathrm{m}} \mathrm{F} 1,6 \mathrm{P}_{2}(\mathrm{mM})$ & $0.021 \pm 0.003$ & $0.040 \pm 0.004^{*}$ \\
Hill coefficient & $1.1 \pm 0.1$ & $2.1 \pm 0.2^{*}$ \\
$\mathrm{~K}_{\mathrm{a}} \mathrm{MgSO}_{4}(\mathrm{mM})$ & $1.0 \pm 0.1$ & $0.9 \pm 0.2$ \\
$\mathrm{I}_{50} \mathrm{~F} 2,6 \mathrm{P}_{2}(\mu \mathrm{M})$ & $1.5 \pm 0.1$ & $3.1 \pm 0.4^{*}$ \\
$\mathrm{I}_{50} \mathrm{AMP}(\mu \mathrm{M})$ & $1.6 \pm 0.3$ & $3.0 \pm 0.7^{*}$ \\
$\mathrm{~V}_{\max }(\mathrm{mU} / \mathrm{mg})$ & $12.3 \pm 0.8$ & $9.3 \pm 0.3^{*}$
\end{tabular}

The data are means $\pm \mathrm{SEM}, \mathrm{n} \geq 4$ independent determinations on separate enzyme preparations. * - Significantly different from the corresponding value for FBPase from the ACR condition using the Student's t-test, $\mathrm{p}<0.05$. 


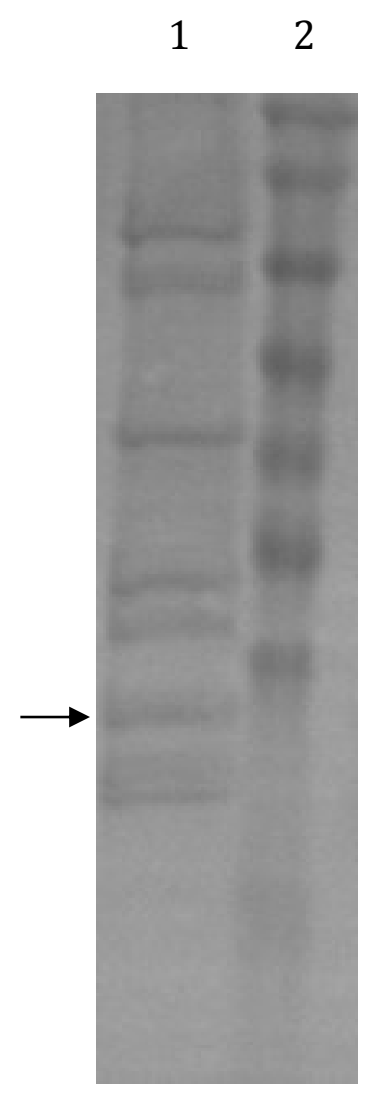

Figure 4.1 Partial purification of skeletal muscle FBPase from the ACR condition. The arrow indicates the band associated with FBPase subunits as identified by commercial FBPase standard loaded on the same gel (not shown). 


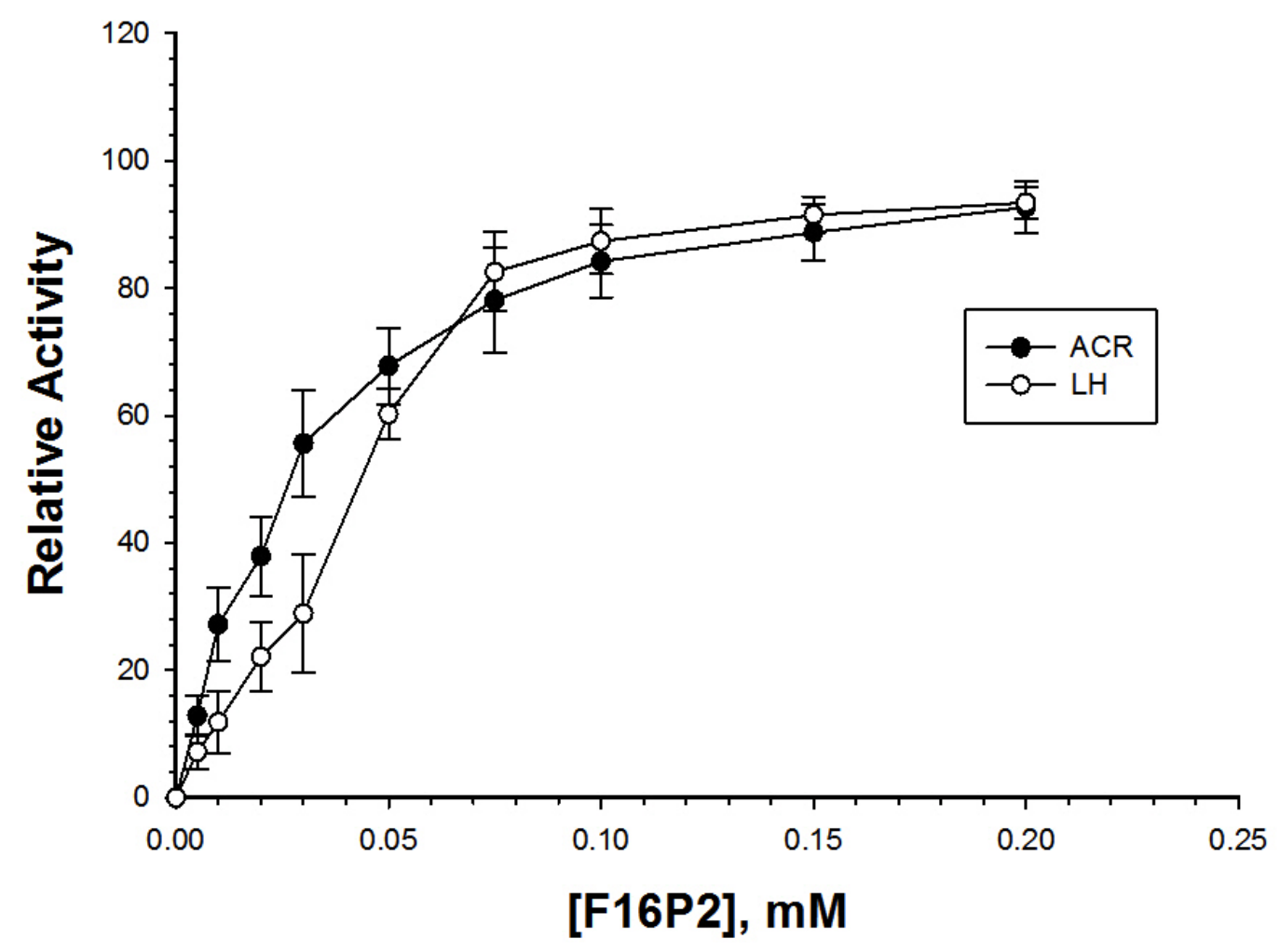

Figure 4.2 Velocity versus $\mathrm{F} 1,6 \mathrm{P}_{2}$ substrate concentration curves for partially purified FBPase derived from ACR and LH muscle samples. Data points are means $\pm S E M, n \geq 4$ independent determinations on separate enzyme samples. 


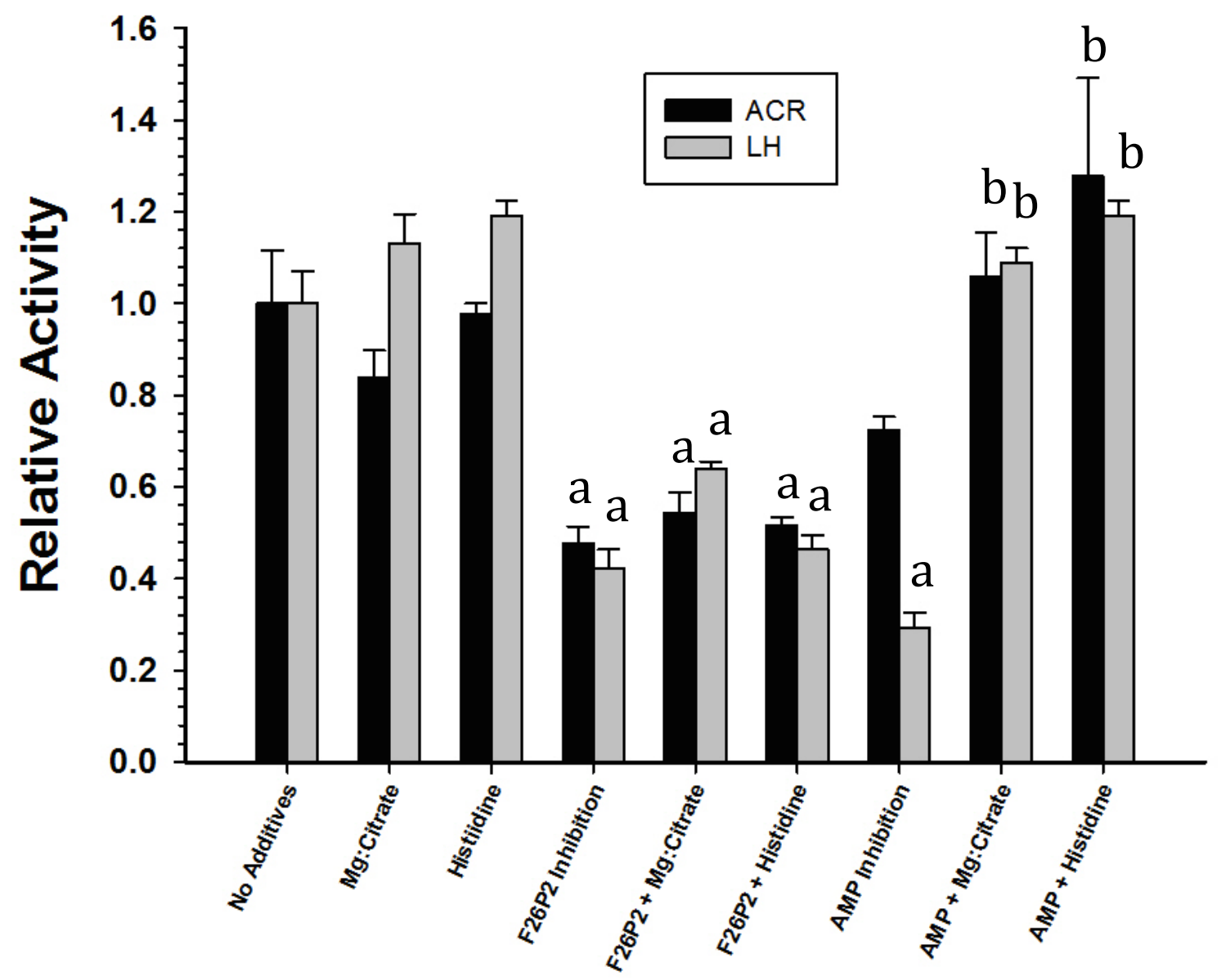

Figure 4.3 Deinhibition of muscle FBPase from ACR and LH conditions with $10 \mathrm{mM}$ Mg:citrate or $10 \mathrm{mM}$ histidine. Activities are expressed relative to the FBPase activity with no inhibitors or deinhibitors being added. Data are means \pm SEM, $n \geq 3$ independent determinations on separate enzyme samples. ${ }^{a}$ - Significantly different from the corresponding value without any additives using the Holm-Sidak method, $\mathrm{p}<0.05{ }^{\mathrm{b}}{ }^{\mathrm{C}}$ Significantly different from the corresponding AMP-inhibited value using the HolmSidak method, $\mathrm{p}<0.05$. 


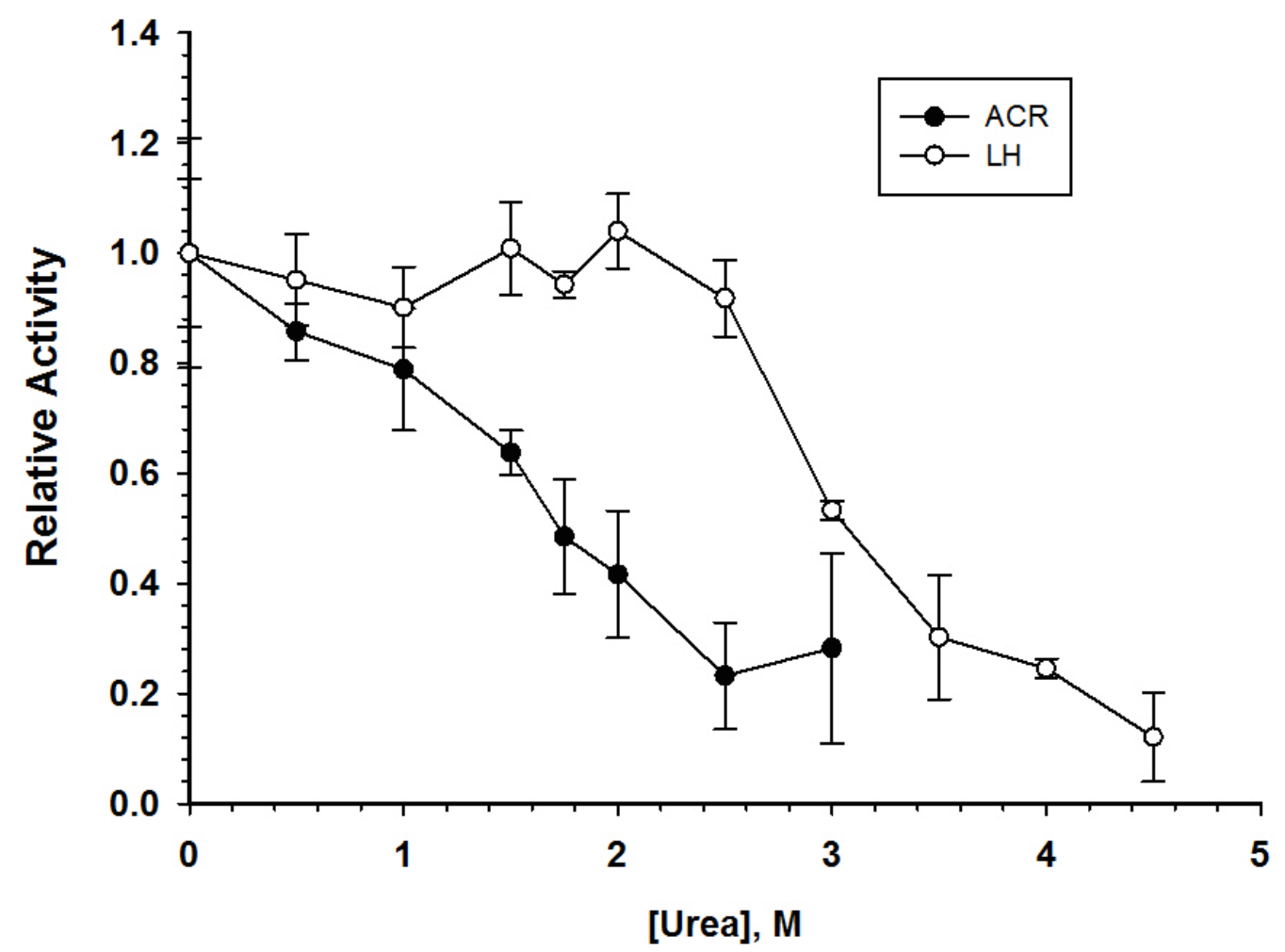

Figure 4.4 Inhibition of muscle FBPase by urea. Data are means $\pm S E M, n \geq 3$ independent determinations on separate enzyme samples. 


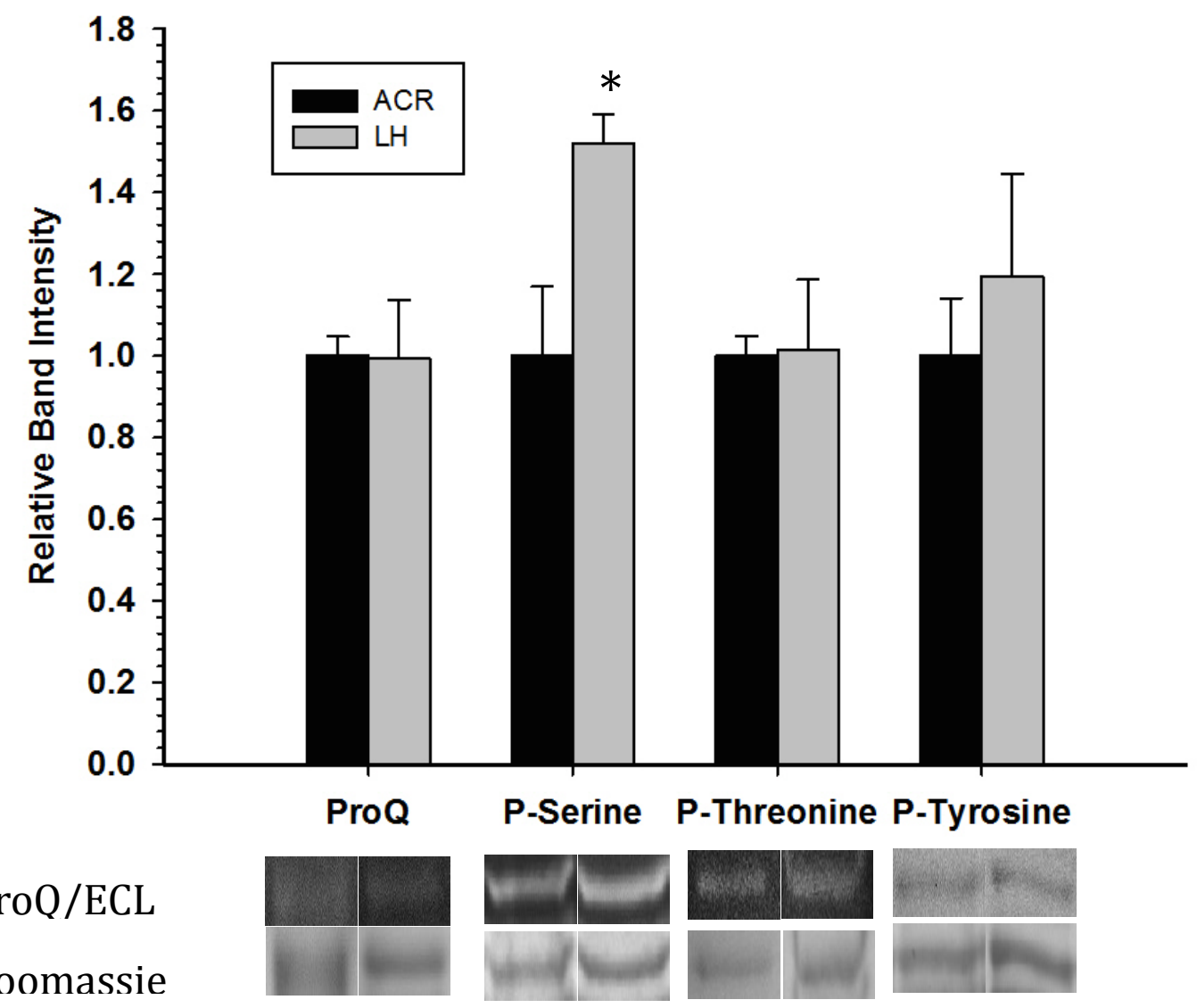

Figure 4.5 Muscle FBPase phosphorylation state. Data are means \pm SEM, $n \geq 4$ independent determinations on separate enzyme samples run on the same gel. * Significantly different from corresponding value from the ACR condition using the Student's t-test, $\mathrm{p}<0.05$. 


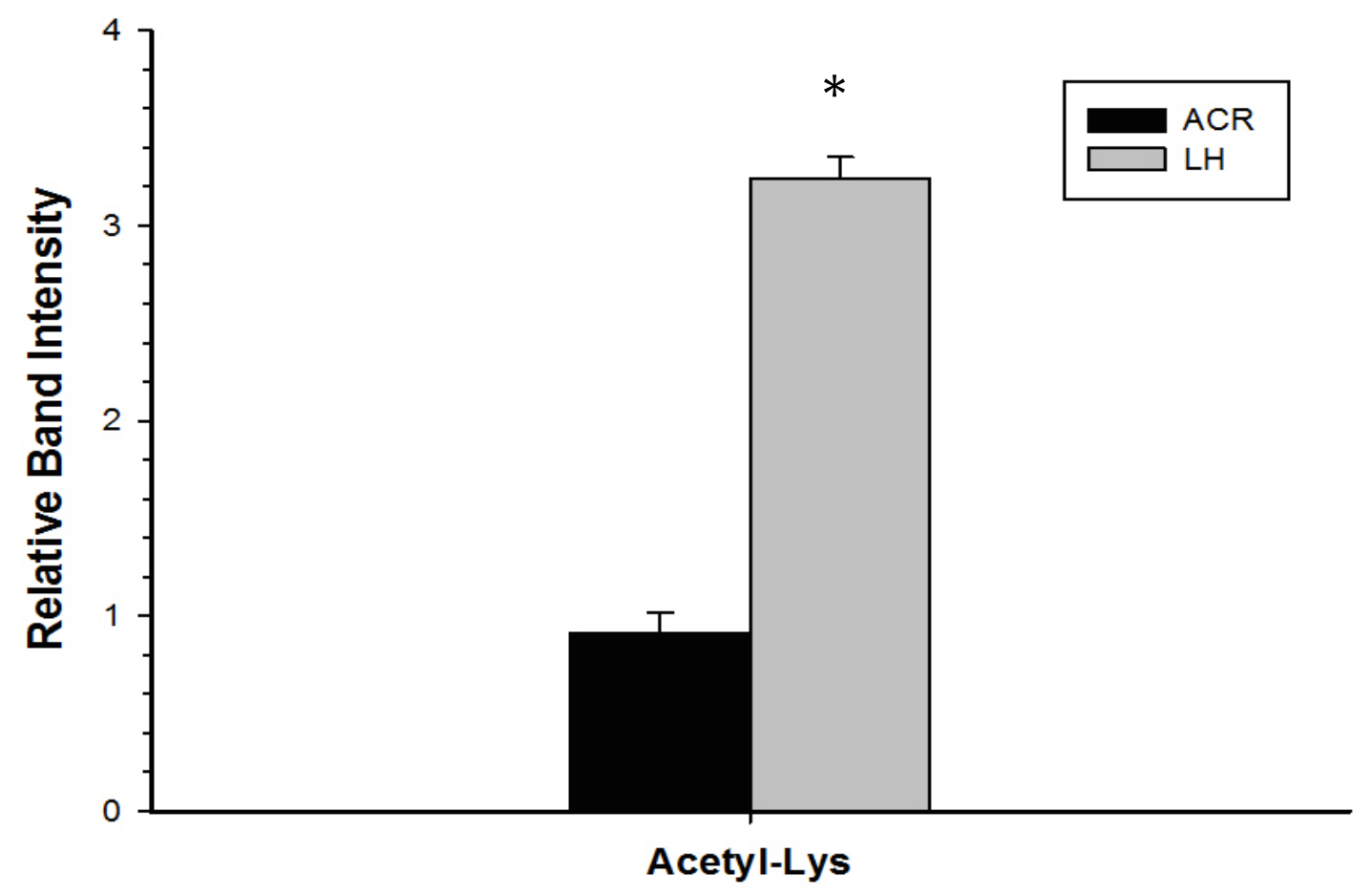

ECL

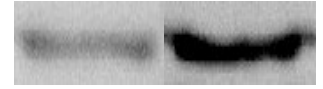

Coomassie

Figure 4.6 Posttranslational acetylation of muscle FBPase. Data are means \pm SEM, $\mathrm{n} \geq 4$ independent determinations on separate enzyme samples run on the same gel. * -

Significantly different from the same value for muscle FBPase from the ACR condition using the Student's t-test, $\mathrm{p}<0.05$. 


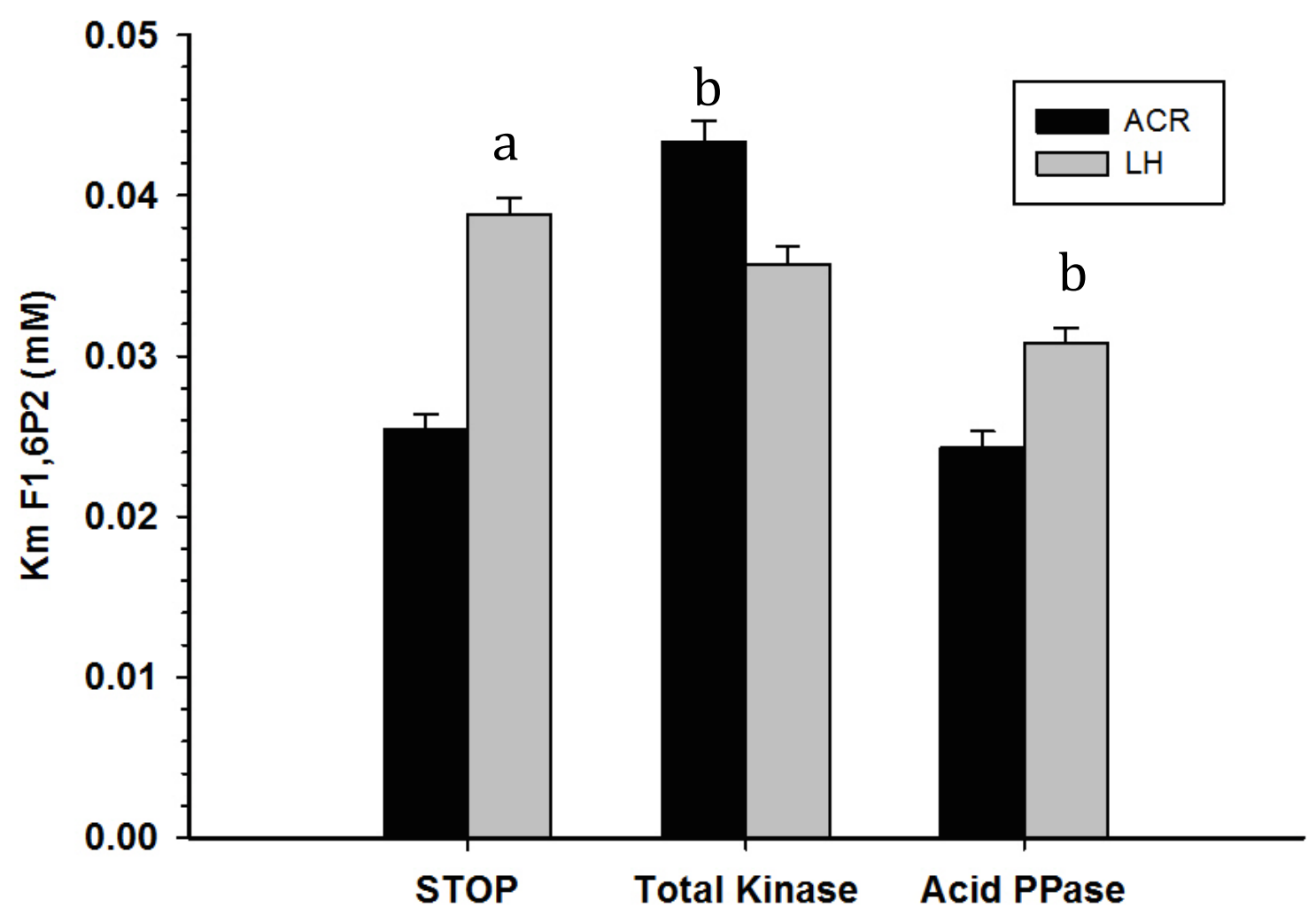

Figure 4.7 The effect of in vitro incubations to stimulate endogenous protein kinases and commercial protein phosphatase on $\mathrm{K}_{\mathrm{m}} \mathrm{F} 1,6 \mathrm{P}_{2}$ for ACR and LH FBPase. The data are means \pm SEM on $n=4$ determinations on separate enzyme samples. ${ }^{a}-$ indicates a significant difference between $\mathrm{K}_{\mathrm{m}} \mathrm{F} 1,6 \mathrm{P}_{2}$ for ACR and LH FBPase under the STOP condition, as determined by the Student's t-test, $p<0.05 .{ }^{\mathrm{b}}$ - indicates a significant difference between the $\mathrm{K}_{\mathrm{m}} \mathrm{F} 1,6 \mathrm{P}_{2}$ values for FBPase and the corresponding STOP condition, as determined by the Dunnett's test, $\mathrm{p}<0.05$. 


\section{Chapter 5}

\section{Regulation of Protein Phosphatase 2A by Reversible Phosphorylation during 13-lined Ground Squirrel Torpor}




\section{Introduction}

The regulation of signaling within a cell is of paramount importance for its survival, as well as the survival of the organism as a whole. The most common mechanism for the propagation of cell signals is through reversible phosphorylation, which is mediated by protein kinases and protein phosphatases. Although kinases have been given much of the attention in most cellular processes, protein phosphatases are highly regulated proteins that play an equally important role in regulating cell signaling.

Protein phosphatase 2A (PP2A) is a ubiquitously expressed serine/threonine phosphatase that typically consists of a catalytic subunit (PP2Ac) as well as two regulatory subunits. The primary regulatory subunit associated with PP2Ac is the $65 \mathrm{kDa}$ subunit PR65 (Mayer-Jaekel and Hemmings, 1994). The second regulatory subunit is highly variable, with at least 18 different proteins separated into four subfamilies that can play this role. These regulatory subunits typically act to localize the holoenzyme to different subcellular sites, determine substrate specificity, and to some degree affect enzyme activity (reviewed in Janssens and Goris, 2001). In contrast to the numerous regulatory subunits, the catalytic subunit exists in only two isoforms, $\alpha$ and $\beta$. The catalytic isoforms share $97 \%$ of their primary amino acid sequence, however, PP2Ac $\alpha$ is expressed about 10-fold more than PP2Ac $\beta$ (Khew-Goodall and Hemmings, 1988; Khew-Goodall et al., 1991). Catalytic subunit activity is known to be regulated by several distinct mechanisms including posttranslational phosphorylation and leucine methylation (Chen et al., 1992; Xie and Clarke, 1994). The role of PP2A within the cell is highly variable being integrally involved in such processes as translation, cell cycle, 
apoptosis, cell metabolism as well as the general stress response (reviewed in Janssens and Goris, 2001).

Entry of ground squirrels into their yearly hibernation cycle, and in particular the transition into and out of torpor, requires careful control of many cellular processes. A wealth of research has indicated that regulation of protein function through reversible phosphorylation is central to controlling long torpor bouts (Storey and Storey, 2010), which puts protein kinases and phosphatases at the forefront of winter survival for the 13lined ground squirrel. The present chapter describes the purification and characterization of the catalytic subunit of protein phosphatase $2 \mathrm{~A}$ from ground squirrel skeletal muscle, with a special focus on the posttranslational modification and regulation of PP2Ac during torpor.

\section{Materials and Methods}

Animals

Animal experiments and tissue isolation was conducted as described in Chapter 2. Purification of the Catalytic Subunit of PP2A

PP2Ac was purified from skeletal muscle of ground squirrels in the ACR and LH stages of hibernation. Frozen skeletal muscle samples were homogenized 1:5 w:v in buffer A (50 mM MES, pH 6.5, 2 mM EDTA, 2 mM EGTA, 10 mM $\beta$-mercaptoethanol, $10 \% \mathrm{v}: \mathrm{v}$ glycerol, with a few crystals of phenylmethylsulphonyl fluoride added just prior to homogenization) using a Polytron homogenizer. Homogenates were spun at 13, $500 \mathrm{~g}$ for 30 minutes at $5^{\circ} \mathrm{C}$, after which supernatants were decanted and held on ice until use in the first purification step. 
Endogenous small molecules, specifically free inorganic phosphate, were removed by passing crude skeletal muscle supernatants through small $(0.5 \times 5 \mathrm{~cm}, \mathrm{~d} \times \mathrm{h})$ columns of G50 Sephadex pre-equilibrated in buffer A. Samples were spun through the column at $2500 \mathrm{RPM}$ for 1 minute. The resulting eluent was collected and mixed 1:2 v:v with $100 \%$ ethanol (Favre et al., 1994). This preparation was placed on a rotator at $4^{\circ} \mathrm{C}$ for 30 minutes, after which the solution was centrifuged at $8000 \mathrm{~g}$ for 10 minutes. The supernatant was discarded and the pellet was resuspended in buffer A $(500 \mu \mathrm{L})$ and held on ice until a DEAE Sephadex column was prepared.

DEAE Sephadex beads were placed into a small column $(1 \times 7 \mathrm{~cm}, \mathrm{~d} \times \mathrm{h})$, and equilibrated in buffer A. The resuspended ethanol-treated muscle extract was placed on top of the bed of DEAE and allowed to seep into the beads. The DEAE Sephadex beads were subsequently washed with buffer A until the absorbance at $280 \mathrm{~nm}$ decreased to the level seen with just buffer, ensuring that all unbound proteins and small molecules were removed. Proteins were eluted using a linear salt gradient of 0-1 $\mathrm{M} \mathrm{KCl}$ made in buffer A, and the peak activity fractions pooled and placed at $4^{\circ} \mathrm{C}$ until further experimentation.

The purity of PP2A catalytic subunit (PP2Ac) was assessed by SDS-PAGE followed by immunoblotting and Coomassie blue staining of the peak fractions from the final purification step, as described later.

PP2Ac Activity Assay

PP2Ac was assayed using a malachite green/ammonium molybdate detection system that measures the amount of free inorganic phosphate released by PP2Ac action, as described by Ekman and Jager (1993). Briefly, all glassware and spatulas were prewashed in $4 \mathrm{M} \mathrm{HCl}$ prior to use. The reagent was prepared by mixing one volume of 
$10 \%(\mathrm{w} / \mathrm{v})$ ammonium molybdate $\left[\left(\mathrm{NH}_{4}\right)_{6} \mathrm{Mo}_{7} \mathrm{O}_{24} \cdot 4 \mathrm{H}_{2} \mathrm{O}\right]$ in $4 \mathrm{M} \mathrm{HCl}$ with 3 volumes of $0.2 \%(\mathrm{w} / \mathrm{v})$ malachite green in $4 \mathrm{M} \mathrm{HCl}$. The solution was covered with tin foil to protect the light-sensitive dye and stirred at room temperature for 30 minutes. The solution was then gravity filtered through Whatman filter paper and stored in a dark bottle covered in tin foil. This reagent was stable for over one month. The red dye turns green when bound to inorganic phosphate.

Initial activity measurements for PP2Ac were determined via the use of paired reaction tubes. PP2Ac activity was calculated as the difference in phosphate production between PP2A-inhibited assays (10 nM okadaic acid added to the assay) and noninhibited PP2A assays. Many repetitions of these assays indicated that, as expected, assays inhibited by okadaic acid (blanks) yielded no noticeable colour change when using the purified enzyme sample. Thus, subsequent PP2Ac kinetic analyses were done with only periodic checks of blanks via the paired reaction system. Assay conditions for maximum PP2Ac activity were obtained by adding $30 \mu \mathrm{L}$ of purified PP2Ac to $480 \mu \mathrm{M}$ of peptide substrate dissolved in Tris- $\mathrm{HCl}, \mathrm{pH} 8.0$ buffer in a final volume of $50 \mu \mathrm{L}$. The peptide substrate used was RRApTVA which is the related to the phosphoacceptor site on pyruvate kinase from several mammalian species, including humans, rat and mouse (Donella-Deana et al., 2003); peptide was purchased from Queen's University Peptide Synthesis Lab. Initial experiments determined the optimal reaction time for the PP2A to be $1 \mathrm{~h}$ (i.e. a $1 \mathrm{~h}$ reaction time produced subsequent colour development in the linear range, thereby ensuring initial reaction rate measurement). Following incubation $750 \mu \mathrm{L}$ of $\mathrm{ddH}_{2} \mathrm{O}$ and $200 \mu \mathrm{L}$ of the malachite green/ammonium molybdate dye system were added, mixed and incubated for an additional 15 minutes (also optimized). It should be 
noted that colour development was always done at room temperature regardless of assay temperature, and was always done in the dark. Aliquots of $200 \mu \mathrm{L}$ were then added to microplate wells and the absorbance at $595 \mathrm{~nm}$ was determined by an MR5000 microplate reader from Dynatech Laboratories (Chantilly, VA) using Biolynx 2.0 software (Brockville, ON). Phosphate production was determined through comparison to a $\mathrm{KH}_{2} \mathrm{PO}_{4}$ standard curve, and activities are presented as nmol phosphate produced $/ \mathrm{min} / \mathrm{mg}$ soluble protein $(\mathrm{U} / \mathrm{mg})$.

Assays at $5^{\circ} \mathrm{C}$ and $37^{\circ} \mathrm{C}$ were conducted with the use of a temperature-controlled incubator set to the respective temperature. Solutions to be used in the reaction were preequilibrated at the given temperature prior to the final mixing of reagents. Reaction mixtures were incubated at the given temperature for $1 \mathrm{~h}$ and then reactions were stopped by the addition of the acidic dye reagent and colour development was completed after a 15 minute incubation at room temperature. Kinetic constants and soluble protein content was determined as previously described in Chapter 2. All assays were performed the same day of enzyme purification.

SDS Polyacrylamide Gel Electrophoresis, ProQ Diamond Phosphoprotein Staining and Immunoblotting

Purified ACR and LH PP2Ac samples were prepared for SDS-PAGE as described in Chapter 2. Western blots that utilized crude skeletal muscle samples were prepared slightly differently than described above for purified enzyme samples. Briefly, frozen muscle samples were homogenized 1:2 w:v in a buffer containing $20 \mathrm{mM}$ HEPES, pH 7.5, $200 \mathrm{mM} \mathrm{NaCl}, 0.1 \mathrm{mM}$ EDTA, $10 \mathrm{mM} \mathrm{NaF}, 1 \mathrm{mM} \mathrm{Na} \mathrm{VO}_{4}, 10 \mathrm{mM} \beta-$ glycerolphosphate, and $1 \mu \mathrm{L}$ of protease inhibitor cocktail (Bioshop). Just prior to 
homogenization a few crystals of the serine protease inhibitor, phenylmethylsulphonyl fluoride, were added to sample. Tissue was homogenized and subsequently centrifuged at $4^{\circ} \mathrm{C}$ for $15 \mathrm{~min}$ at $10,000 \mathrm{x}$. Supernatants were collected and soluble protein concentrations determined using the Bradford assay with the Bio-Rad prepared reagent. Samples were standardized to a constant protein concentration and then mixed 1:1 v:v with 2 x SDS sample buffer (as described in Chapter 2).

Gel electrophoresis, ProQ Diamond phosphoprotein staining and immunoblotting on purified and crude samples were conducted as described in Chapter 2. Notable changes included the use of $3 \mu \mathrm{L}$ of FroggaBio PINK Plus Prestained protein ladder added to one lane of every gel to provide molecular weight markers. Antibodies additional to those listed in Chapter 2 were: (1) PP2Ac $\alpha$ (Genscript), (2) phospho-Y307 PP2Ac $\alpha / \beta$ (Santa Cruz Biotechnology), and (3) Methyl-arginine (Covalab). PP2Ac Homology Modeling and Peptide Docking

In order to construct a three-dimensional model of PP2Ac from 13-lined ground squirrels, the amino acid sequence was obtained from the Ensembl bioinformatics database (ENSSTOG00000010560) and used to search known crystallographic structures of PP2Ac. This search was completed in the Molecular Operating Environment (MOE), which subsequently identified the protein databank file 2IE3.c (PP2Ac from humans) as the highest quality match. Thirteen-lined ground squirrel PP2Ac was modeled after the human PP2Ac structure within MOE under the AMBER99 forcefield (used for simulating protein conformations; Cornell et al., 1995). The resulting structure was validated using the Ramachandran plot and used for peptide docking experiments. 
Prior to peptide docking to PP2Ac both the 13-lined ground squirrel model and the peptide (RRApTVA - synthesized in MOE) were protonated and energy minimized. Once this was completed, MOE Site Finder was used to locate the potential active site for PP2Ac. Several possibilities emerged but the one chosen as the most likely location of the active site contained the following amino acid residues: Arg89, Asn117, His118, Ile123, Tyr127, Trp200, and Arg214. This site was chosen as the active site for two reasons: (1) it was one of two sites that contained the essential residue His118, which is known to be part of the active site of PP2Ac (Myles et al., 2001); and (2) when docking the peptide substrate to each of the aforementioned sites the site identified above yielded the lowest energy docking interaction. Following the docking simulation within MOE, the most energetically favoured peptide substrate conformer was chosen as the correct orientation for the substrate in the chosen active site. This docking experiment was performed again following the phosphorylation of Y307 on 13-lined ground squirrel PP2Ac and an energy minimization of the altered structure. Again, the most energetically favourable peptide substrate conformation for docking into the chosen active site was taken as the correct substrate orientation for phospho-PP2Ac. Protein ligand interactions, docking energetics, and physical orientation of the peptide were analyzed for the phospho- and dephospho-PP2Ac structures to determine the effects of Y307 phosphorylation on peptide interaction.

\section{Results}

PP2Ac Purification and Kinetics

The catalytic subunit of PP2A was isolated through the use of ethanol precipitation and DEAE ion exchange chromatography (Fig. 5.1). PP2Ac was purified to 
electrophoretic homogeneity with a final yield of 67\% (Fig 5.2; Table 5.1). Fig. 5.2

confirms the purification by showing that only a single immunoreactive PP2Ac band occurred and that this was also the sole band seen after Coomassie blue staining. Compared with the molecular mass standards, the PP2Ac band showed a size of approximately $36 \mathrm{kDa}$, the expected size for mammalian PP2Ac. It should be noted that although only the PP2Ac $\alpha$ antibody was used, cross-reaction of this antibody with $\mathrm{PP} 2 \mathrm{Ac} \beta$ is possible and cannot be ruled out. For this reason, this study refers to the isolation and characterization of PP2Ac and not a particular isoform of the enzyme.

Once purified, PP2Ac kinetic properties were assessed at $5^{\circ} \mathrm{C}$, room temperature $\left(22^{\circ} \mathrm{C}\right)$, and $37^{\circ} \mathrm{C}$. At room temperature and $37^{\circ} \mathrm{C}$ the $\mathrm{K}_{\mathrm{m}}$ peptide was $60 \%$ and $52 \%$ higher, respectively, for LH PP2Ac as compared to the $\mathrm{K}_{\mathrm{m}}$ peptide for PP2Ac from the euthermic condition (Table 5.2). Conversely, the $\mathrm{K}_{\mathrm{m}}$ peptide at $5^{\circ} \mathrm{C}$ was not significantly different between torpid and euthermic conditions. With respect to the maximal PP2Ac activity, only the activity of PP2Ac at room temperature was significantly different between the two conditions; LH PP2Ac activity at $22^{\circ} \mathrm{C}$ was significantly higher as compared to ACR (Table 5.2).

PP2Ac Posttranslational Modification

Several PP2Ac posttranslational modifications were investigated in this study in an attempt to identify potentially important modifiers of PP2A function. Principal among these was reversible phosphorylation. PP2Ac is well known to be phosphorylated at Y307, and analysis of crude skeletal muscle samples indicated that LH PP2Ac was approximately $50 \%$ more phosphorylated on that residue as compared to the ACR control enzyme (Fig. 5.3A). Crude skeletal muscle samples were also assessed for total PP2Ac 
levels and no changes were observed between ACR and LH conditions (Fig. 5.3A). Following purification, total PP2Ac phosphorylation levels were assessed via ProQ Diamond phosphoprotein staining and no significant changes were observed between ACR and LH conditions (Fig. 5.3B). In addition to phosphorylation, PP2Ac acetylation was assessed for purified PP2Ac samples, with the result being a near $70 \%$ decrease in subunit acetylation during torpor as compared to control samples (Fig. 5.4). Lastly, PP2Ac isolated from either ACR or LH conditions did show lysine and arginine methylation of the protein, however, no significant difference was observed between ACR and LH conditions with respect to their overall level of methylation (Fig. 5.4). PP2Ac Homology Model and Peptide Docking

The homology model created through MOE had an associated Ramachandran plot which showed limited structural outliers ( $<1 \%$ of amino acids are outliers; Appendix IE). These outliers were not found within the template structure, although the template PDB 2IE3.c was truncated by 5 and 16 amino acid residues at the $\mathrm{N}$-terminal and $\mathrm{C}$-terminal ends of the protein, respectively (not shown). Aside from the truncated portions of the protein, 13-lined ground squirrel and human amino acid sequences showed 100\% identity. Taken together the limited number of structural outliers and the overall identity between model and template was taken to indicate that the model was an acceptable structure for PP2Ac.

Simulated docking of the peptide substrate that was used for in vitro enzymatic assays to the dephosphorylated form of PP2Ac yielded a docking interaction of -57.29 $\mathrm{kcal} / \mathrm{mol}$. In comparison, docking between peptide substrate and phospho-PP2Ac yielded a docking interaction of $-27.84 \mathrm{kcal} / \mathrm{mol}$. The physical orientation of the peptide 
substrate with respect to dephosphorylated PP2Ac situates the phosphate moiety deep in the active site pocket (indicated in red in Fig. 5.5A) with suspected direct interactions with Arg214 and Asn117 (Fig. 5.5B). Additionally, the peptide substrate in the dephosphorylated PP2Ac active site appears to be in a conformation that is well suited for the available pocket with numerous potential contact points on PP2Ac. Upon phosphorylation of Y307 on PP2Ac it appears that the active site binds to the peptide substrate such that the phosphate moiety is oriented away from active site amino acids (Fig. 5.6A), with no direct interactions with any active site amino acids (Fig. 5.6B). The peptide substrate also appears to fit less well within the active site pocket and appears to have considerably less interaction with PP2Ac than occurs for the dephosphorylated protein.

\section{Discussion}

Establishing a state of aerobic hypometabolism as a strategy for winter survival requires a significant reorganization of cellular processes. A large portion of this readjustment is mediated by protein kinases and phosphatases, which control the phosphorylation state and thereby the activity of many cell proteins. PP2A is a ubiquitous serine/threonine phosphatase that plays an essential role in many aspects of cellular function (Virshup, 2000; Lechward et al., 2001). The present study investigates the kinetic and physical characteristics of PP2Ac within ground squirrel skeletal muscle, and proposes a potential role for PP2A in regulating carbohydrate metabolism during torpor.

Purification of PP2Ac from ground squirrel muscle used a combination of ethanol precipitation and low-pH anion exchange chromatography. This represents a novel 
method for PP2Ac isolation. Ethanol stripping of PP2Ac from its regulatory subunits and the concomitant precipitation of the catalytic subunit has been reported in previous purification schemes (Favre et al., 1994), however, the low-pH DEAE Sephadex step is completely novel. The latter step takes advantage of the low isoelectric point of PP2Ac (predicted to be 5.3 using ExPaSy Compute pI/MW tool)(Bjellqvist et al., 1993;

Bjellqvist et al., 1994; Gasteiger et al., 2005) in that PP2Ac is expected to retain some negative charge at $\mathrm{pH} 6.5$ when most other proteins become positively charged and therefore repel the similarly charged DEAE resin. This was evidenced by the large amount of protein that was lost in the wash fractions prior to DEAE chromatography (Fig. 5.1). Furthermore, when protein began eluting from the column in response to increasing concentrations of salt, PP2Ac bound more strongly to the DEAE column than most other proteins (i.e. PP2Ac eluted late off the column in comparison to other bound proteins). The overall yield of PP2Ac from ground squirrel skeletal muscle was $67 \%$, and with such simple steps this purification scheme represents a significant improvement on previous purification procedures that are far more laborious (Lee and Stock, 1993; Favre et al., 1994).

Once isolated, PP2A kinetics were investigated at $5^{\circ} \mathrm{C}, 22^{\circ} \mathrm{C}$ and $37^{\circ} \mathrm{C}$. At $22^{\circ} \mathrm{C}$ and $37^{\circ} \mathrm{C} \mathrm{K}$ peptide was significantly higher for PP2Ac purified from the hibernating condition as compared to PP2Ac from the euthermic condition (Table 5.2). This trend was maintained at $5^{\circ} \mathrm{C}$ but the increase in $\mathrm{K}_{\mathrm{m}}$ peptide during torpor was not significant. A change in PP2Ac $\mathrm{K}_{\mathrm{m}}$ peptide was also seen in a study of another hibernating mammal, the Richardson's ground squirrel, where torpor induced a decrease in peptide affinity at $5^{\circ} \mathrm{C}$ and room temperature (Wu et al., 2013). The aforementioned study also linked these 
kinetic changes to an increase in PP2Ac phosphorylation at tyrosine-307, which is known to regulate PP2Ac activity inside the cell (Chen et al., 1992). Similarly, this study shows that there is a significant increase in PP2Ac phosphorylation at this residue during torpor (Fig. 5.3A), even though detection of overall PP2Ac phosphorylation by ProQ Diamond phosphoprotein staining indicated no difference in global phosphorylation of the enzyme between torpid and euthermic conditions (Fig. 5.3B). The latter result does not necessarily contradict the result from the pY307-specific Western blots as differences in phosphorylation state at this specific site may be too small to detect via ProQ Diamond. Furthermore, since ProQ Diamond detects global phosphorylation levels, it is possible that an overall assessment of PP2Ac phosphorylation will allow other phosphorylation events to potentially mask site-specific changes. Indeed, PP2Ac is known to be phosphorylated on a number of other residues, including Y248, Y265, Y284, and T304 (Gu et al., 2010; Zhou et al., 2013); many of these phosphorylation events were detected in various cell carcinomas and it is not known whether they are relevant to normal cellular function of the enzyme.

Phosphorylation at Y307 on PP2Ac is generally accepted to be negative modulator of PP2Ac activity, however it is unknown if/how this modification affects substrate binding. Results from the present experiments suggest that phosphorylation at Y307 may decrease the affinity of the active site for its protein substrate (Table 5.2), and computational methods were then used in attempt to corroborate or contradict this notion. Docking simulations, conducted using MOE, between the PP2Ac homology model and the peptide substrate used in this study indicated that Y307 phosphorylation led to a near $60 \%$ increase in binding energy as compared to the substrate binding energy for 
dephosphorylated PP2Ac. Furthermore, analysis of the ligand interaction with the PP2Ac active site suggested that when Y307 is phosphorylated the peptide substrate is unable to make many contacts with the active site (Fig. 5.5B and 5.6B). Phosphorylation at Y307 also seems to rotate the phosphate moiety on the substrate away from the active site possibly making cleavage more difficult (Fig. 5.5A and 5.6A). The increase in binding energy and decrease in PP2Ac ligand interactions coincides with the increase in $\mathrm{K}_{\mathrm{m}}$ peptide observed in this study, and suggests that mechanistically, Y307 phosphorylation significantly alters protein substrate binding.

It is important to note that the alteration to substrate binding identified through the above mentioned simulation does not necessarily reflect the true situation in vivo. As stated previously, PP2Ac is known to have numerous phosphorylation sites as well as other known posttranslational modifications, which could affect substrate binding or catalytic activity in a negative or positive way. The results merely demonstrate that phosphorylation at Y307 alone appears to be capable of altering substrate binding and may be the overwhelming reason for the altered $\mathrm{K}_{\mathrm{m}}$ peptide observed between PP2Ac from the ACR and LH conditions.

The potential targets for PP2A that are currently known are substantial and continue to grow, making investigations into the downstream targets and effects of PP2A in hibernation difficult. One known target that is thought to be specifically dephosphorylated by PP2Ac is FOXO1 (Yan et al., 2008). This transcription factor, which is involved in gluconeogenesis and glycogenolysis, has also been analyzed in 13lined ground squirrel muscle and found to be more highly phosphorylated during torpor as compared to the euthermic control situation (Hapsatou and Storey, unpublished). 
Enhanced phosphorylation of any protein target, including FOXO1, can be achieved by either increasing protein kinase action or inhibiting protein phosphatase. Hence, an inhibition of PP2A action due to decreased substrate affinity of ground squirrel muscle PP2Ac in the torpid condition (probably due to enhanced phosphorylation of PP2A at Y307) would fit well with the known torpor-dependent changes FOXO1. PP2A is also known to dephosphorylate both pyruvate kinase (PK) and fructose-1,6-bisphosphatase (FBPase) (Cohen, 1985; Donella-Deana et al., 2003). Earlier work in this thesis on 13lined ground squirrel skeletal muscle PK and FBPase fits well with decreased PP2Ac activity during torpor, since both enzymes were found to be more highly phosphorylated during torpor.

In an attempt to further characterize structural modifications and potential regulatory elements of PP2Ac, other posttranslational modifications, in addition to phosphorylation, were investigated. For instance, Western blot analyses found that the catalytic subunit of PP2A from control and torpid conditions contained methylated lysine and arginine residues. Methylation is a known modification of PP2Ac, however, previous studies have mainly focused on methylation on leucine-309 (Reviewed in Janssens and Goris, 2001). Thus, the PP2Ac lysine methylation identified in the current study appears to be novel, although there was no significant difference in lysine- or arginine-methylation of PP2Ac between ACR and LH conditions. The function of lysine modification is unknown, however, regulation through reversible lysine methylation has been shown to modify protein stability, activity, localization, and/or interactions with other proteins (Zhang et al., 2012). Further experiments would need to be devised to 
probe the function of PP2Ac methylation, but it does not seem to be a key regulator of PP2Ac activity within ground squirrel muscle.

PP2Ac posttranslational acetylation was also investigated to determine if this covalent modification may be an important regulator of PP2Ac function during torpor. PP2Ac from the torpid condition was approximately $70 \%$ less acetylated as compared to the acetylation of the euthermic control enzyme (Fig. 5.4). Acetylation has been previously reported for the PP2A catalytic subunit, however no function has yet been associated with this modification (Choudhary et al., 2009). Further experimentation would be necessary to elicit the role of acetylation, but simply the difference between control and torpid conditions suggests it may have a significant role during torpor.

In conclusion, skeletal muscle PP2Ac was suppressed during ground squirrel torpor, with the suspected mechanism being a significant increase in Y307 phosphorylation. Computational simulations support this proposition that Y307 phosphorylation is responsible for the decreased affinity of the PP2Ac active site for its peptide substrate. This may have implications for maintaining a suppressed state of carbohydrate metabolism during ground squirrel torpor by inhibiting PP2A from acting on proteins such as PK, FBPase and FOXO1. Additionally, PP2Ac was found to be posttranslationally modified by a non-phosphate moiety with a significant decrease in the level of acetylation of PP2Ac during torpor as compared to the control condition. The function of this modification is currently unknown but may represent a significant regulatory mechanism that can modify protein stability, protein-protein interactions, or protein localization during ground squirrel torpor. 
Table 5.1. Purification of PP2Ac from skeletal muscle of ACR 13-lined ground squirrels.

$\begin{array}{llllll}\text { Purification Step } & \text { Total } & \text { Total } & \text { Specific } & \text { Fold } & \text { \% Yield } \\ & \begin{array}{l}\text { Protein } \\ (\mathrm{mg})\end{array} & \text { Activity } & \text { Activity } & \text { Purification } & \\ & & (\mathrm{U} / \mathrm{mg}) & & \end{array}$

\begin{tabular}{llllll}
\hline Crude & 10.4 & - & - & - & - \\
Sephadex G50 & 8.2 & 430 & 52 & - & 100 \\
Ethanol & 7.5 & 305 & 40 & 0.8 & 71 \\
$\begin{array}{l}\text { Precipitation } \\
\text { DEAE pH 6.5 }\end{array}$ & 0.055 & 286 & 5200 & 100 & 67
\end{tabular}

One unit is defined as the amount of enzyme necessary for the production of $1 \mathrm{nmol}$ of $\mathrm{Pi}$ per minute. 
Table 5.2. Kinetic properties of purified PP2Ac from skeletal muscle of 13-lined ground squirrels from ACR and LH conditions.

\begin{tabular}{llll|lll} 
& \multicolumn{3}{c}{$\mathrm{ACR}$} & \multicolumn{4}{c}{$\mathrm{LH}$} \\
& $5^{\circ} \mathrm{C}$ & $22^{\circ} \mathrm{C}$ & $35^{\circ} \mathrm{C}$ & $5^{\circ} \mathrm{C}$ & $22^{\circ} \mathrm{C}$ & $35^{\circ} \mathrm{C}$ \\
\hline $\begin{array}{l}\mathrm{K}_{\mathrm{m}} \text { Peptide } \\
(\mu \mathrm{M})\end{array}$ & $162 \pm 2$ & $150 \pm 24$ & $230 \pm 30$ & $190 \pm 16$ & $240 \pm 30^{*}$ & $350 \pm 47^{*}$ \\
$\mathrm{~V}_{\max }$ & & & & & \\
$(\mathrm{nmol} / \mathrm{min} / \mathrm{mg})$ & $3.6 \pm 0.3$ & $7 \pm 1$ & $20.1 \pm 0.5$ & $4.2 \pm 0.3$ & $13 \pm 1^{*}$ & $25 \pm 2$
\end{tabular}

Data are means \pm SEM, $\mathrm{n} \geq 4$ independent determinations on individually isolated samples. * Significantly different from the corresponding value for the ACR condition using the Student's t-test, $\mathrm{p}<0.05$. 


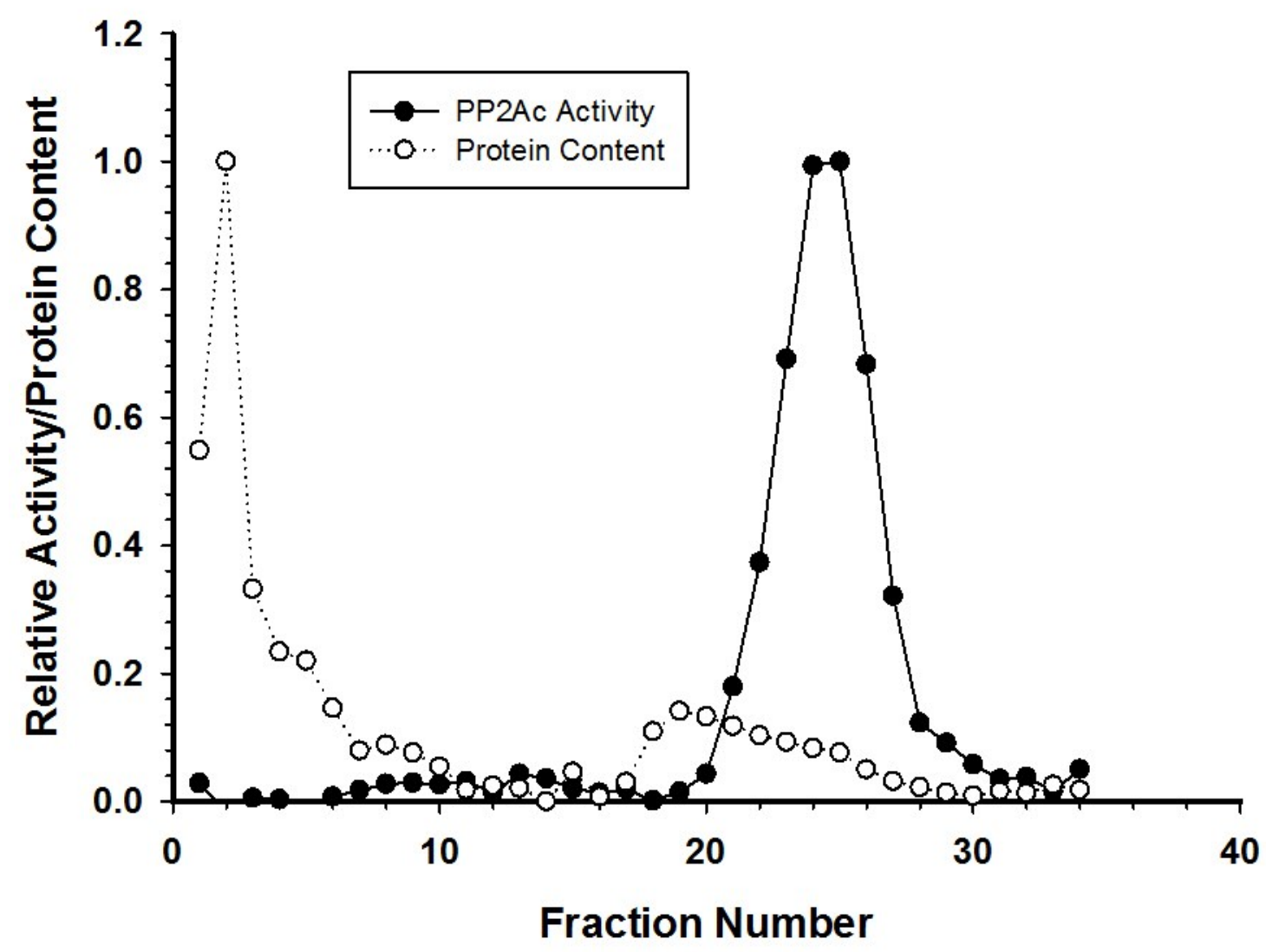

Figure 5.1 Representative elution profile for ACR PP2Ac off of a DEAE-Sephadex column. The protein content in each fraction as well as the PP2Ac activity are overlaid on this graph. Fractions 1-10 are wash fractions prior to starting the $0-1 \mathrm{M} \mathrm{KCl}$ gradient. 

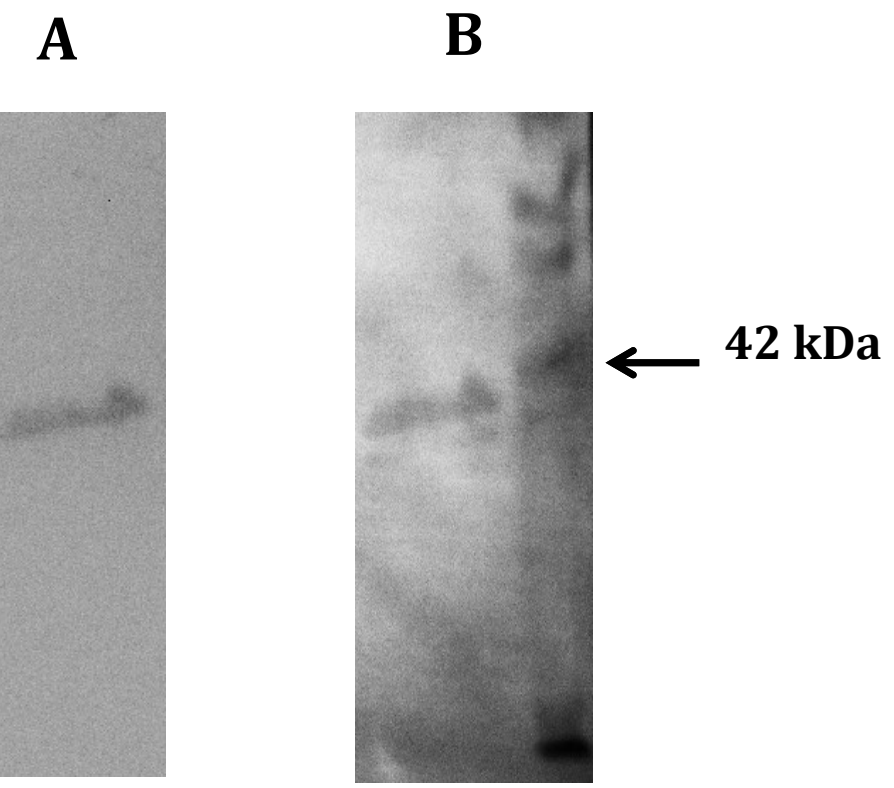

Figure 5.2 Purification of PP2Ac from the skeletal muscle of ACR 13-lined ground squirrels. (A) Western blot showing the PP2A $\alpha$ catalytic subunit; (B) Coomassie blue stained membrane of Western blot depicted in (A). The protein ladder is seen on the right hand side of (B) with the band at $42 \mathrm{kDa}$ indicated via an arrow. 
A

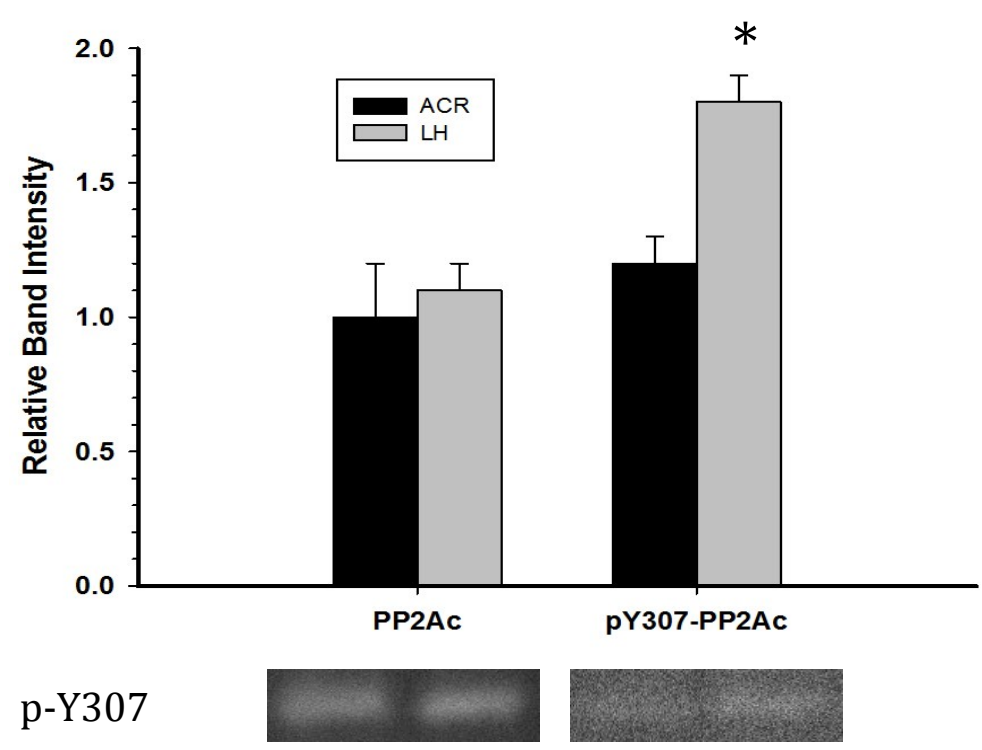

B

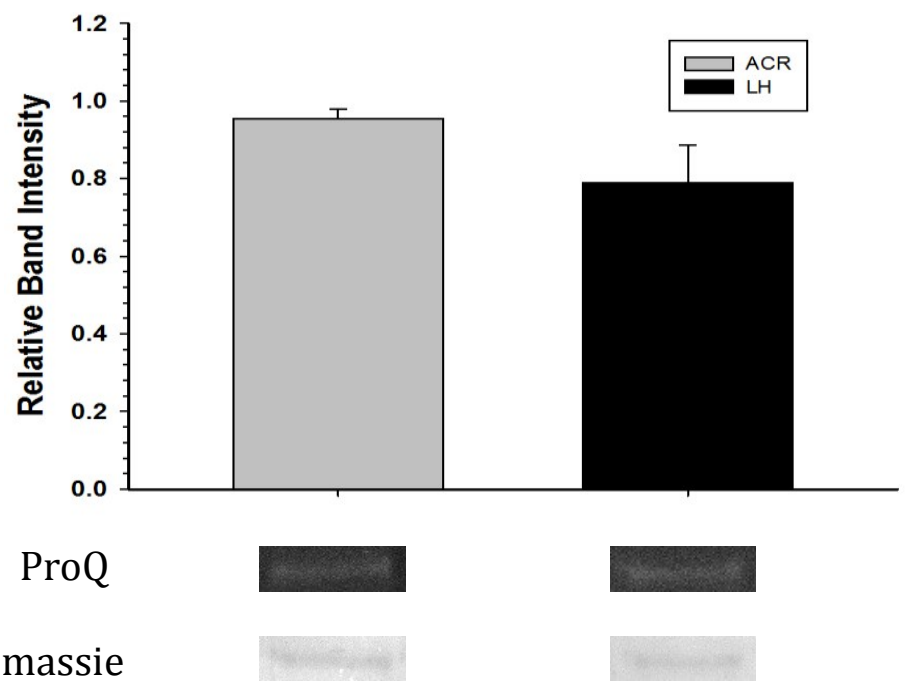

Figure 5.3 Relative levels of 13-lined ground squirrel PP2Ac and phospho-PP2Ac in ACR and LH skeletal muscle preparations. (A) Total PP2Ac and phospho-Y307 PP2Ac relative levels in crude muscle preparations. Representative immunoblot bands are also shown. (B) ProQ Diamond phosphoprotein staining of purified PP2Ac from skeletal muscle with representative bands for ProQ and Coomassie blue staining. Data are means \pm SEM, $\mathrm{n} \geq 4$ individually prepared enzyme samples. * - Significantly different from the corresponding ACR value using the Student's t-test. 


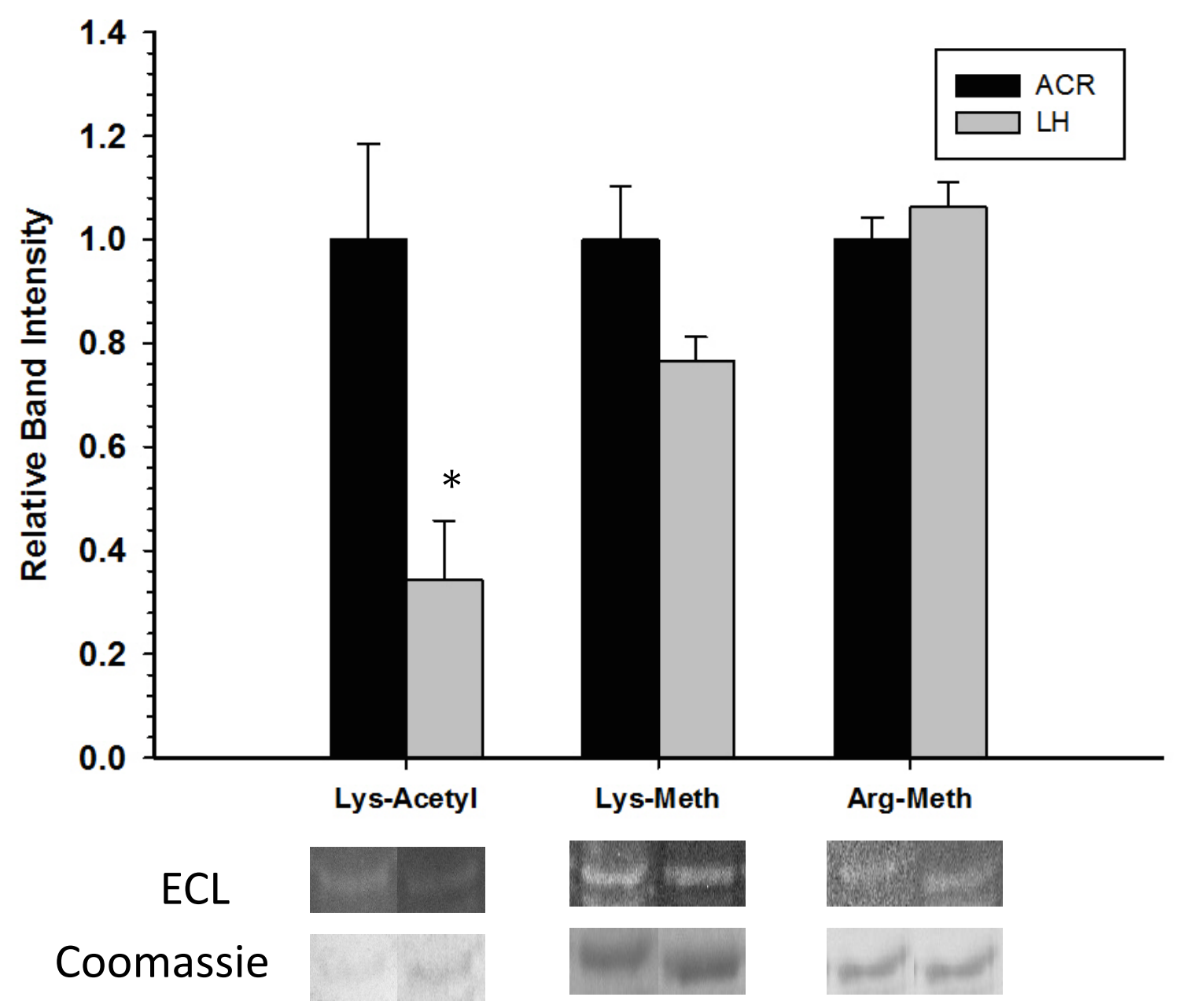

Figure 5.4 Posttranslational modifications of PP2Ac from skeletal muscle of ACR and LH conditions: acetylation, lysine methylation and arginine methylation. Representative immunoblots are shown along with Coomassie staining of the same bands. Data are means $\pm \mathrm{SEM}, \mathrm{n}=4$ individually purified enzyme samples run on the same gel/blot. * Significantly different from the corresponding ACR value using the Student's t-test, $\mathrm{p}<0.05$. 
A

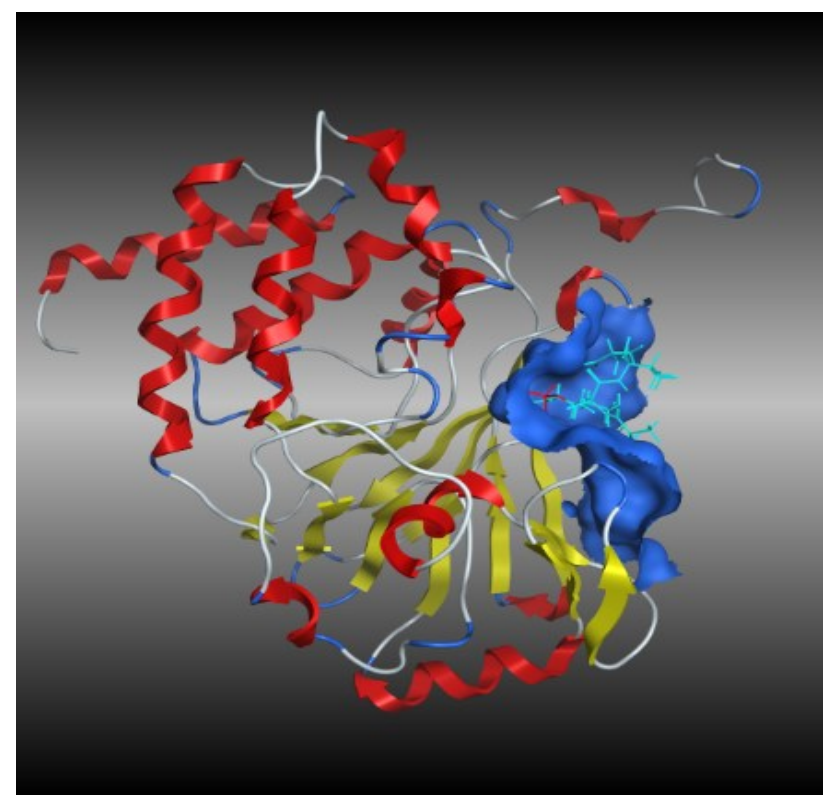

B

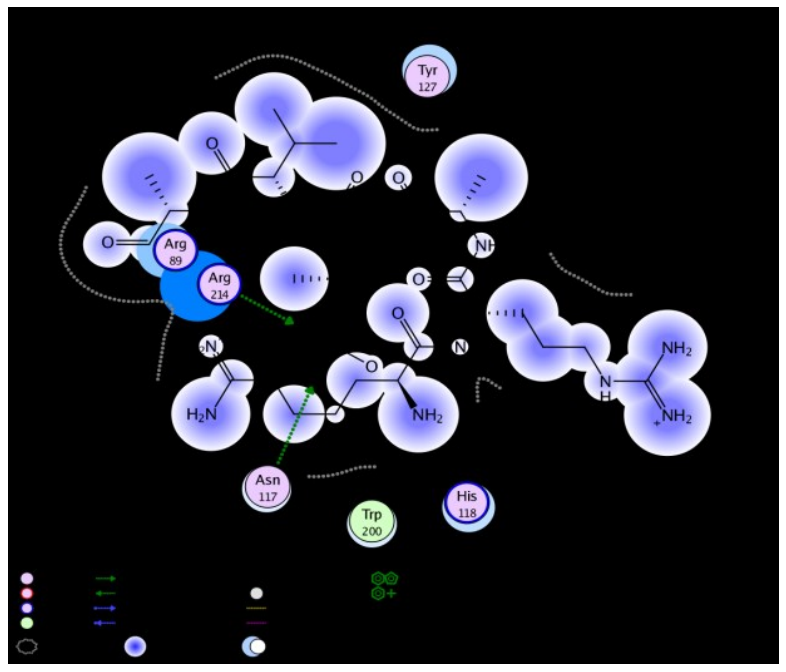

Figure 5.5 Peptide docking to the dephosphorylated PP2Ac homology model produced from the 13-lined ground squirrel amino acid sequence. (A) Homology model depicting the orientation of the peptide substrate (RRApTVA; light blue) within the PP2Ac active site. The atoms coloured in red indicate the phosphate moiety on the aforementioned peptide substrate. (B) Ligand interactions between the dephospho-PP2Ac active site and the peptide substrate. 
A

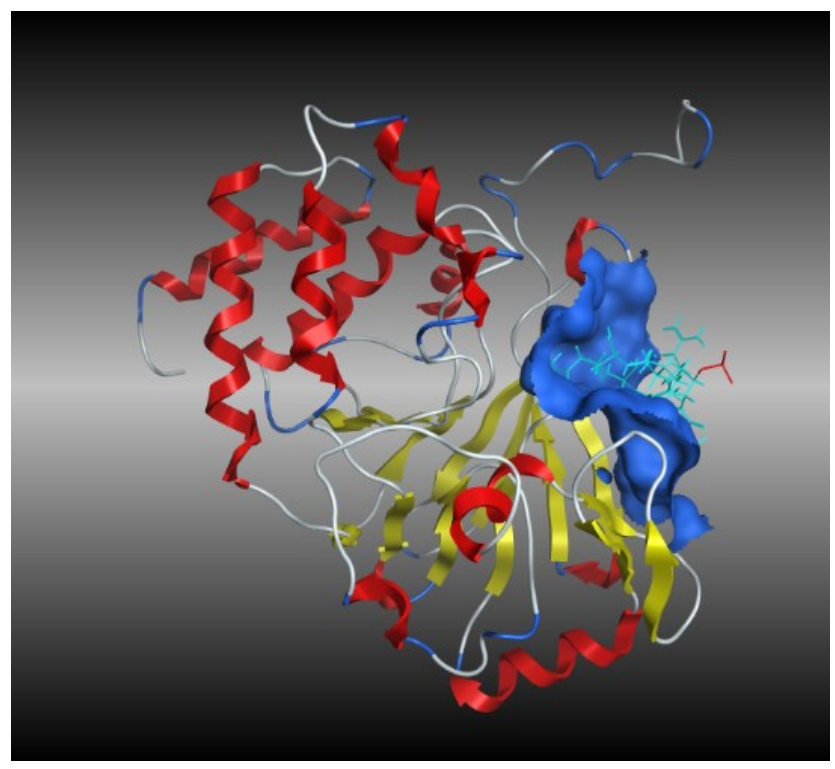

B

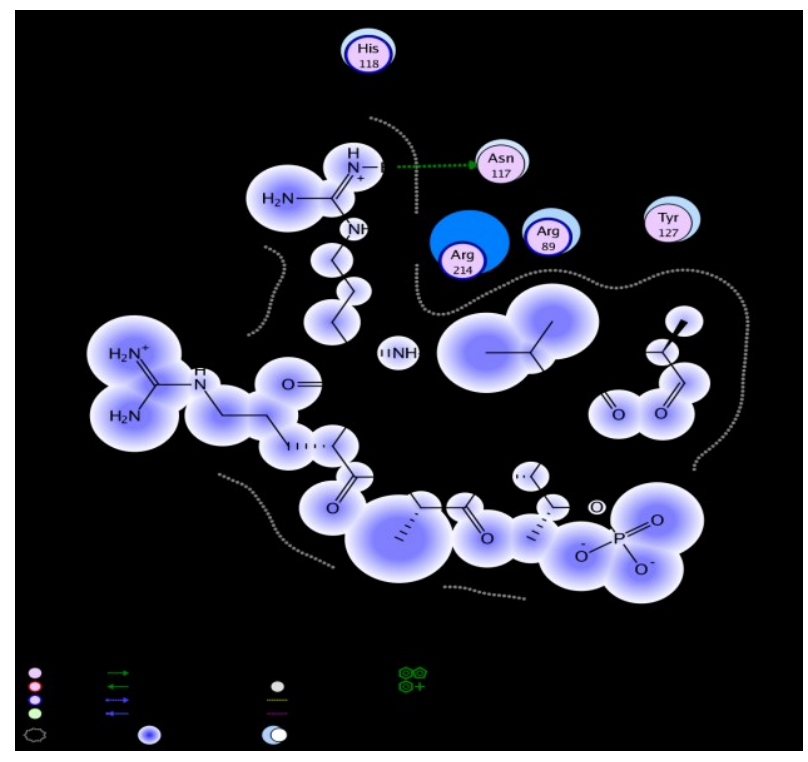

Figure 5.6. Peptide docking to phosphorylated Y307-PP2Ac homology model produced from the 13-lined ground squirrel amino acid sequence. (A) Homology model depicting the orientation of the peptide substrate (RRApTVA; light blue) within the pY307-PP2Ac active site. The atoms coloured in red indicate the phosphate moiety on the aforementioned peptide substrate. (B) Ligand interactions between the phospho-PP2Ac active site and the peptide substrate. 


\section{Chapter 6}

Regulation of skeletal muscle glycogen synthase kinase-3 from the hibernating thirteen-lined ground squirrel. 


\section{Introduction}

As presently understood, the vast majority of cell signaling involved in metabolic control is mediated by protein phosphatases, which catalyze the removal of a phosphate moiety from a protein, and protein kinases, which catalyze the transfer of the $\gamma$ phosphoryl group from ATP onto specific sites on a protein (MacDonald, 2004). Changes in the phosphorylation state of proteins provide the means to alter cellular metabolic state and function in response to changing environmental conditions by providing the link between the extracellular environment and internal cellular processes. Hence, animal exposure to major changes in food availability, oxygen, water, or temperature can initiate systemic effects that cause protein kinases to propagate signals for cell survival.

Thirteen-lined ground squirrels encounter many of the conditions mentioned above during harsh northern winters, and protein kinase function helps to establish a state of aerobic torpor that allows for ground squirrel survival. Torpor requires changes to many cellular processes, such as transcription, translation, cell cycle, membrane trafficking, and metabolism (reviewed in Carey et al., 2003), all of which require the action of select protein kinases.

Glycogen synthase kinase-3 (GSK3) is a very important protein kinase in the cell that is involved in regulating a variety processes such as intermediary metabolism (Alessi et al., 1996), the cell cycle, apoptosis (Ferkey and Kimelman, 2002), and cytoskeletal dynamics (Hanger et al., 1992; Doble and Woodgett, 2003). GSK3 has two isoforms, GSK $3 \alpha$ and GSK3 3 , both of which have nearly identical kinase domains but differ substantially in their N-terminal and C-terminal ends (Force and Woodgett, 2009). While many of the functions overlap between GSK $3 \alpha$ and GSK3 $\beta$, several studies 
suggest that there may be tissue-specific roles for each isoform (MacAuley et al., 2007; Patel et al., 2008). The canonical regulatory role of GSK3 is, of course, the phosphorylation and inactivation of glycogen synthase (Rylatt et al., 1980), the crucial enzyme gating carbohydrate storage as glycogen and acting in opposition to glycogen phosphorylase. The literature to date is somewhat unclear as to the state of skeletal muscle glycogen stores during mammalian hibernation, with several studies indicating a decrease in glycogen during torpor (Zimny and Tyrone, 1957; Galster and Morrison, 1970; Galster and Morrison, 1975), whereas others indicate an increase or maintenance of glycogen levels (Hannon and Vaughn, 1961; el Hachimi et al., 1990; James et al., 2013). Given this discrepancy and the importance of GSK3 in regulating various metabolic processes including glycogen synthesis, this study investigated the kinetic and regulatory properties of skeletal muscle GSK3 during ground squirrel torpor.

\section{Materials and Methods}

Animals

Animal origin, treatment and tissue excision was conducted as described in Chapter 2.

\section{GSK3 Purification}

Samples of frozen skeletal muscle tissue from 13-lined ground squirrels were homogenized 1:5 v:v in homogenization buffer $(25 \mathrm{mM}$ Tris- $\mathrm{HCl}, \mathrm{pH} 8.0,2.5 \mathrm{mM}$ EDTA, $2.5 \mathrm{mM}$ EGTA, $15 \mathrm{mM} \beta$-mercaptoethanol, $25 \mathrm{mM} \mathrm{NaF}$, and 10\% v:v glycerol). The resulting homogenate was centrifuged at $13,500 \mathrm{~g}$ for 30 minutes at $5^{\circ} \mathrm{C}$. The supernatant was then isolated and applied to a DEAE Sephadex column $(6 \times 1 \mathrm{~cm}, \mathrm{~h} \times \mathrm{d})$ pre-equilibrated in homogenization buffer. The column was subsequently washed with 
$20 \mathrm{~mL}$ of homogenization buffer to remove any unbound proteins, after which a $0-2 \mathrm{M}$ $\mathrm{KCl}$ gradient in homogenization buffer was used to elute bound proteins.

The location of GSK3 within the DEAE Sephadex fractions was determined by Western blotting using 10\% resolving gels prepared as described in Chapter 2 and DEAE fractions prepared in SDS loading buffer (as defined in Chapter 2). Equal volumes of sample from each fraction were loaded onto gels, then run and blotted onto PVDF paper as previously described. Blots were then subsequently probed with GSK3- $\alpha / \beta$ antibody (Santa Cruz Biotechnology) diluted 1:1000 v:v in TBST. Subsequent application of secondary antibody and chemiluminescent detection was as described in Chapter 2. Fractions shown to contain GSK3 were then pooled and held on ice until an affinity column could be prepared.

A blue-agarose column $(3 \times 1 \mathrm{~cm} ; \mathrm{h} \times \mathrm{d})$ was equilibrated in homogenization buffer prior to the application of the pooled DEAE fractions containing GSK3. This column was then washed with $30 \mathrm{~mL}$ of homogenization buffer to remove unbound proteins, and then proteins were eluted with a $0-2 \mathrm{M} \mathrm{KCl}$ gradient in homogenization buffer. Western blots were again used to identify the fractions that contained GSK3. Finally, those fractions from the blue-agarose column that contained GSK3 were pooled and applied to a phospho-cellulose column $(3 \times 2 ; \mathrm{h} \times \mathrm{d})$ that had been preequilibrated in homogenization buffer. The column was then washed with $30 \mathrm{~mL}$ of homogenization buffer to rid the column of unbound proteins. GSK3 was then eluted with a $20 \mathrm{mM}$ bump of Mg-ATP in homogenization buffer and the presence of GSK3 in the bump fractions was again determined with Western blots. 
The purity of the final GSK3 preparation was assessed by Western blotting after concentrating the enzyme in centrifugal filters (10 kDa cut-off; Amicon). Specifically, centrifugal filters were spun at $8000 \mathrm{RPM}$ for $20 \mathrm{~min}$ at $5^{\circ} \mathrm{C}$ in a Sorvall centrifuge. GSK $3 \alpha / \beta$ and GSK3 $\beta$ (Santa Cruz Biotechnology) primary antibodies, as well as Coomassie brilliant blue staining of the PVDF membranes were used to detect the presence of GSK3 and assess purity.

Development and Validation of the Kinase-Glo Assay for use with the Chemigenius Bioimaging System

The Kinase-Glo luminescent kinase assay is typically performed with the help of a spectrophotometer capable of detecting a luminescent signal. This study, however, utilized a ChemiGenius Bioimaging system that had not previously been used for this assay platform. ATP standard curves were created using both the Cary Eclipse Fluorescence Spectrophotometer (Agilent Technologies, ON, Canada) and the ChemiGenius Bioimaging System (Syngene, MD, USA) to determine if this detection system was valid for the Kinase-Glo assay. Using either machine, reaction mixtures contained $20 \mathrm{mM} \mathrm{MgCl}$, and 0-100 $\mu \mathrm{M}$ ATP in Tris- $\mathrm{HCl}, \mathrm{pH} 7.5$, made up to a final volume of $50 \mu \mathrm{L}$ in a 96-well Costar opaque black microplate. Equal volumes of KinaseGlo Plus reagent was added to the wells containing ATP and these solutions were allowed to sit for 10 minutes to allow for the luminescent signal to stabilize. Detection using the Cary Eclipse spectrophotometer involved selecting single read mode for a luminescent signal at $550 \mathrm{~nm}$ (experimentally determined luminescent $\lambda_{\max }$ ) and detecting that signal for $1 \mathrm{~s}$. In contrast, utilizing the ChemiGenius Bioimaging system, no specific 
filter was chosen and luminescence was measured for $1 \mathrm{~s}$. Accompanying GeneTools software was used to measure the luminescence of each well.

Following the production of the standard curves, GSK3 was assayed using the ChemiGenius Bioimaging system to assess whether kinase activity could be effectively detected via this method. Assays were conducted at $22^{\circ} \mathrm{C}$ with $50 \mu \mathrm{M}$ ATP. Further details for the kinase assay are described below.

GSK3 Assay and the Kinase-Glo Luminescent Kinase Assay Platform

Partially purified skeletal muscle GSK3 (after the blue-agarose step) was used in kinetic assays involving the Kinase-Glo luminescent kinase platform. Specifically, those fractions that contained GSK3 were pooled and concentrated in centrifugal filters (as described above). The resulting concentrate ( $\sim 50 \mathrm{X}$ more concentrated than the pooled fractions) was then used in GSK3 assays, which contained $25 \mathrm{mM}$ Tris- $\mathrm{HCl}, \mathrm{pH}$ 7.5, 50 $\mu \mathrm{M}$ ATP, $20 \mathrm{mM} \mathrm{MgCl}$, and varying amounts of GSK3 peptide [YRRAAVPPSPSLSRHSSPHQ(pS)EDEEE; SignalChem] ranging from 0-185 $\mu \mathrm{M}$. The peptide is phosphorylated at the indicated serine residue since GSK3 will preferentially phosphorylate substrates that are previously phosphorylated by other kinases. The underlined serine residues in the peptide sequence can both be phosphorylated by GSK3 (Rylatt et al., 1980). Selected assays also contained $1 \mathrm{mM} \mathrm{LiCl}$ that was used to inhibit GSK3 activity and provide a negative control (Klein and Melton, 1996; Stambolic et al., 1996). Assays were attempted in 96-well (final reaction volume was $50 \mu \mathrm{L}$ ) Costar black microplates, and kinase reactions were allowed to proceed for $3.5 \mathrm{~h}$ at $5^{\circ} \mathrm{C}$ or $1 \mathrm{~h}$ at $37^{\circ} \mathrm{C}$. Reactions were stopped by the addition of the Kinase-Glo reagent and then incubated for 10 min to stabilize the luminescent signal. Luminescence was detected as described 
above for the ATP standard curve. All enzyme preparations were used on the same day as purification.

GSK3 Activity and $K_{m}$ Calculation

Luminescent units measured by the GeneTools software were converted to the amount of ATP remaining after the kinase reaction using the standard curve and then the amount of ATP utilized by the kinase reaction was calculated. This value was then divided by the assay time and the amount of protein present in each assay, as measured by the Bradford assay (described in Chapter 2). Final activities were expressed as nmol ATP utilized per minute per mg soluble protein. These activity measurements were then used to determine the $\mathrm{K}_{\mathrm{m}}$ peptide and $\mathrm{V}_{\max }$ with the help of the Kinetics v.3.5.1 computer program (Brooks, 1992).

Immunoblotting

Immunoblots utilizing crude skeletal muscle samples were carried out as described in Chapter 2 and Chapter 5 (details of the sample preparation). Aliquots of samples containing $20 \mu \mathrm{g}$ of protein were loaded into the wells of a 10\% SDS gel, electrophoresed and then wet transferred onto a PVDF membrane, as previously outlined. Membranes were incubated with one of the following primary antibodies (diluted 1:1000 v:v in TBST): (1) GSK3 $\alpha / \beta$ (Santa Cruz Biotechnology), (2) GSK3 $\beta$ (Santa Cruz Biotechnology), (3) phospho-Ser9 GSK3 $\beta$ (Santa Cruz Biotechnology), (4) glycogen synthase (Biorbyt), or (5) phospho-Ser641/645 glycogen synthase (Millipore). Primary antibodies were allowed to incubate overnight at $4{ }^{\circ} \mathrm{C}$, followed by incubation with secondary antibody and then enhanced chemiluminescence detection as described previously in this thesis. 


\section{Results}

\section{GSK3 $\beta$ Purification}

A single isoform of GSK3 was purified to electrophoretic homogeneity using a combination of DEAE-Sephadex, Blue-agarose and phospho-cellulose column chromatography (Fig. 6.1A). Western blot analysis of the final preparation indicated that this band corresponded to GSK3 $\beta$ (Fig. 6.1B). It is important to note that GSK3 $\alpha$ was never detected in any preparations analyzed in this study, including Western blot analyses of crude skeletal muscle preparations (data not shown).

\section{Development and Validation of the Kinase-Glo Assay for use with the ChemiGenius}

\section{Bioimaging System}

GSK3 activity was detected using the Kinase-Glo luminescent kinase assay with the luminescent signal being measured by a ChemiGenius Bioimaging system, which has not been used for this purpose previously. A standard curve for the luminescent signal generated when varying concentrations of ATP (between 0 and $100 \mu \mathrm{M}$ ) were present

yielded a linear line when using either the ChemiGenius Bioimaging system $\left(\mathrm{R}^{2}=0.999\right.$; Fig 2A) or the Cary Eclipse spectrophotometer $\left(\mathrm{R}^{2}=0.994\right.$; Fig $\left.2 \mathrm{~B}\right)$. Partially purified GSK3 (taken after the blue-agarose step) from euthermic ground squirrels was then used to generate a velocity versus peptide substrate concentration curve to validate that kinase activity could be reliably produced using this novel detection method (Fig. 6.3A and 6.3B). The $\mathrm{K}_{\mathrm{m}}$ peptide that resulted from these experiments was $39 \pm 4 \mu \mathrm{M}$. Kinase-Glo Luminescent Kinase Assay and GSK3 Activity

After establishing the validity of this detection system, GSK3 kinetic parameters for the partially purified enzyme were determined with $50 \mu \mathrm{M}$ ATP, and at two different 
temperatures: $5^{\circ} \mathrm{C}$ and $37^{\circ} \mathrm{C}$. At $5^{\circ} \mathrm{C}, \mathrm{K}_{\mathrm{m}}$ peptide did not change between euthermic and torpid conditions (Table 6.1). However, at this low temperature, GSK3 from the torpid condition displayed just $55 \%$ of the $\mathrm{V}_{\max }$ activity of GSK3 derived from euthermic skeletal muscle (Table 6.1). Similar to the situation at $5^{\circ} \mathrm{C}$, assays at $37^{\circ} \mathrm{C}$ revealed that there was no significant difference in $\mathrm{K}_{\mathrm{m}}$ peptide for GSK3 between control and torpid conditions, however, the $\mathrm{V}_{\max }$ for GSK3 from the torpid condition was only $23 \%$ of that seen for GSK3 from the ACR condition (Table 6.1).

\section{GSK3 and GS Immunoblotting}

Initial immunoblotting using an anti-GSK $3 \alpha / \beta$ antibody and crude skeletal muscle extracts indicated that GSK3 $\alpha$ was not present in appreciable amounts in ground squirrel skeletal muscle, and that GSK3 $\beta$ was the primary isoform present (Data not shown). Western blot analysis of the crude skeletal muscle preparations determined that levels of GSK3 $\beta$ were nearly identical between euthermic and torpid conditions (Fig. 6.4). Similar experiments determining the tissue content of phosphorylated GSK3 $\beta$ (at serine 9) indicated that the relative amount of phospho-GSK3 $\beta$ from skeletal muscle of torpid ground squirrels was almost 4-fold higher than that seen in the euthermic animal (Fig. $6.4)$.

Both total and phosphorylated contents of glycogen synthase were also quantified by immunoblotting. There was no significant difference in the expression level of glycogen synthase, however the level of phospho-GS (serine 641/645) was nearly 2-fold higher for GS from the animals during torpor as compared to the enzyme derived from euthermic animals (Fig. 6.5). 


\section{Discussion}

Ground squirrel torpor during winter hibernation is a period that sees significant changes to many biochemical processes to allow for cell survival. Many of these processes rely on the action of protein kinases to mediate flux through important cellular pathways. GSK3 is a protein kinase that is essential to the regulation of many cellular events, not least of which is the regulation of glycogen synthase and the production of cellular glycogen. The state of this key skeletal muscle energy store is unclear during ground squirrel hibernation and thus this study investigated the role of GSK3 in regulating skeletal muscle glycogen synthesis during dormancy.

Prior to assessing GSK3 activity within ground squirrel skeletal muscle, the use of the novel ChemiGenius Bioimaging detection system had to be validated. The first step in assessing assay suitability is to determine the linear dynamic range for the assay using the ChemiGenius Bioimaging system as compared to that observed with the Carey Eclipse spectrophotometer (i.e. a standard luminometer). The Kinase-Glo Plus assay platform claims to have a linear dynamic range from 0-100 $\mu \mathrm{M}$ ATP and this is reflected in standard curves created using both machines (i.e. both standard curves elicited $\mathrm{R}^{2}$ values above 0.99; Fig. 6.2).

Given the linearity of the Kinase-Glo Plus assay platform, initial kinetic analyses were undertaken for GSK3 to determine the suitability of the ChemiGenius Bioimaging system for kinase assays. Partially isolated GSK3 from the skeletal muscle of 13-lined ground squirrels produced a $\mathrm{K}_{\mathrm{m}}$ peptide of $39 \pm 4 \mu \mathrm{M}$ at room temperature $\left(22^{\circ} \mathrm{C}\right.$; Fig. 6.2). The $\mathrm{K}_{\mathrm{m}}$ obtained in this study is similar to that determined for wood frog skeletal muscle GSK3, which utilized radioactive ATP and subsequent detection of radiolabelled 
peptides on a phosphor screen (Dieni et al., 2012). In addition to the seemingly accurate $\mathrm{K}_{\mathrm{m}}$ curve, the ChemiGenius Bioimaging system provided a unique ability to visualize the end point luminescence after the kinase reaction (Fig. 6.3B). This advantage over traditional luminometers allows for well-by-well assessment of experimental error, and for the potential to correct those errors in real-time. For instance, eliminating small bubbles, that would otherwise be unseen in luminometers, increases the accuracy and precision of luminescent measurements.

As indicated above, purification of GSK3 was necessary for any kinetic assessment of this kinase using the Kinase-Glo luminescent kinase assay. Few studies have purified non-recombinant GSK3 from tissue samples, but examples of successful methods included the use of immobilized axin (Primot et al., 2000), as well as a combination of precipitation, ion exchange, and affinity columns somewhat similar to that seen in this study (Hemmings et al., 1981). That being said, the purification scheme of DEAE-Sephadex/Blue-agarose/phospho-cellulose presented in this study does appear to be unique and represents a novel scheme for the isolation of native GSK3. It should be noted that a fold purification and \% yield was not attempted as determination of GSK3 activity was not possible in the crude state or following DEAE chromatography.

Fully purified samples were not used for the kinetic assessment of GSK3 as the number of assays from individual purifications was prohibitively low. Rather, kinetic assessment of GSK3 was conducted on DEAE-Sephadex/Blue-agarose purified enzyme samples. It is important to note that kinetic analyses of GSK3 are presented in this study as though both isoforms could be involved, although it is quite likely that GSK3 $\beta$ is the only isoform being measured given the absence of GSK3 $\alpha$ detection in crude 
preparations. Investigation of GSK3 activity at high and low temperatures $\left(5^{\circ} \mathrm{C}\right.$ and $37^{\circ} \mathrm{C}$ ) indicated that partially purified skeletal muscle GSK3 from the torpid condition was significantly less active as compared to its counterpart from the euthermic state. Specifically, the $\mathrm{V}_{\max }$ values for torpid GSK3 were $44 \%$ and $76 \%$ less than the corresponding $\mathrm{V}_{\max }$ values for ACR GSK3 at $5^{\circ} \mathrm{C}$ and $37^{\circ} \mathrm{C}$, respectively (Table 6.1). This finding coincides with previous findings for artic ground squirrel brain GSK3 that indicated decreases in GSK3 activity for torpid ground squirrels as compared to summer active animals ( $\mathrm{Su}$ et al., 2008). However, the current findings in this study are counter to those found in several other animals that are capable of entering into hypometabolic states (Ramnanan et al., 2007; Dieni et al., 2012), one of which, the freeze-tolerant wood frog, displayed an increase in skeletal muscle GSK3 activity during freezing. Given the differences in species and cellular stresses endured between wood frog and ground squirrel there is little basis to refute the results presented in this chapter. It does appear however, that GSK3 is regulated in a tissue- and species-specific manner.

Decreased GSK3 activity typically coincides with an increase in GSK3 phosphorylation at Ser9 (Sutherland et al., 1993; Stambolic and Woodgett, 1994; Cross et al., 1995; Frame et al., 2001), and this was indeed the case in this study. Although total GSK3 levels remained constant (Fig. 6.4), phosphorylation at Ser9 increased strongly during torpor. Ser9 phosphorylation is one of the main regulatory mechanisms for GSK3 control across species, and the principal upstream kinase that carries this out is Akt (protein kinase B). A measure of Akt activation is the level of Ser473 phosphorylation (Alessi et al., 1997; Sarbassov et al., 2005), which has been determined previously in 13lined ground squirrel muscle. The studies by Cai and colleagues (2004) and Wu and 
Storey (2012b) indicated that Akt is less phosphorylated at Ser473 and therefore less active during ground squirrel torpor. The potential decrease in Akt activity in ground squirrel skeletal muscle runs counter to the results dictated here (i.e. a significant increase in GSK3 phosphorylation), which suggests that alternative kinases may be regulating GSK3 in vivo. Indeed, serine 9 can be phosphorylated by other protein kinases including protein kinase A (Fang et al., 2000), p70S6 kinase, p90rsk-1 (Sutherland et al., 1993) and PKC (Ballou et al., 2001; Fang et al., 2002). Although a different species entirely, work on bat skeletal muscle PKA indicated that this protein kinase remained substantially active during hibernation (Holden and Storey, 1998). Furthermore, p70S6 kinase, which is principally involved in regulating protein synthesis, remains equally as active during late torpor as it does during euthermia making it another potential regulator of GSK3 (Wu and Storey, 2012). It should be noted as well that GSK3 contains other known phosphorylation sites that are similarly linked to decreased activity, however, the most prevalent hypothesis is that these phosphorylation events likely lead to phosphorylation at Ser9 (Ding et al., 2005; Thornton et al., 2008).

A main link between GSK3 and metabolism is its role in regulating glycogen synthase (GS). GS expression was observed to remain relatively constant between euthermic and torpid conditions, while phospho(ser641/645)-GS expression increases significantly during torpor (Fig. 6.5). While this coincides well with the suspected decrease in gluconeogenesis and glycogenesis during torpor, it runs counter to the results from this study that indicated a decreased capability of GSK3 to function during dormancy (Fig. 6.4). Although GS phosphorylation sites S641 and S645 are generally thought to be specific sites for GSK3 action (Skurat and Roach, 1995), several studies 
have indicated other kinases that may be responsible for GS regulation at those sites. For instance, Skurat and Dietrich (2004) demonstrate that rabbit skeletal muscle GS is phosphorylated at Ser640 (synonymous with Ser641) by one or several members of the dual-specificity tyrosine phosphorylated and regulated protein kinase (DYRK) family. This phosphorylation event causes a sharp decrease in skeletal muscle GS activity similar to that seen when GSK3 phosphorylates the same site. Moreover, a stress-activated protein kinase (SAPK2b) was found to phosphorylate GS at Ser644 (synonymous with Ser645)(Kuma et al., 2004). These studies indicate that GS phosphorylation at Ser641 and Ser645 are not exclusive to GSK3, and that the increased phosphorylation of GS during ground squirrel torpor may be the result of non-GSK3 protein kinase action.

GSK3 from the skeletal muscle of the 13-lined ground squirrel displayed an increased phosphorylation state at Ser9 during torpor, with a concomitant decrease in enzyme activity at $5^{\circ} \mathrm{C}$ and $37^{\circ} \mathrm{C}$ as compared to GSK3 from the control animal. This decrease in GSK3 activity during torpor did not correlate with GS phosphorylation levels during torpor, suggesting alternative protein kinase regulators for this enzyme during ground squirrel dormancy. That being said, the increase phosphorylation of GS within ground squirrel skeletal muscle does suggest that glycogen synthesis is reduced during torpor. 
Table 6.1. ACR and LH 13-lined ground squirrel skeletal muscle GSK3 kinetics.

\begin{tabular}{clll} 
& Parameter & ACR & LH \\
\hline $\mathbf{5}^{\circ} \mathbf{C}$ & $\mathrm{K}_{\mathrm{m}}$ Peptide $(\mu \mathrm{M})$ & $28.8 \pm 0.7$ & $26 \pm 4$ \\
& $\mathrm{~V}_{\max }(\mathrm{U} / \mathrm{mg})$ & $0.9 \pm 0.1$ & $0.50 \pm 0.02^{*}$ \\
$\mathbf{3 7}^{\circ} \mathbf{C}$ & $\mathrm{K}_{\mathrm{m}}$ Peptide $(\mu \mathrm{M})$ & $26 \pm 5$ & $24 \pm 7$ \\
& $\mathrm{~V}_{\max }(\mathrm{U} / \mathrm{mg})$ & $16 \pm 2$ & $3.7 \pm 0.4^{*}$
\end{tabular}

Assays were performed with $50 \mu \mathrm{M}$ ATP. One unit is defined as the amount of enzyme necessary to utilize $1 \mathrm{nmol}$ ATP per minute. Data are means \pm SEM, $\mathrm{n} \geq 3$ independent determinations on individually purified (partial; DEAE/Blue agarose) enzyme samples. * - Significantly different from the ACR value using the Student's t-test, $p<0.05$. 

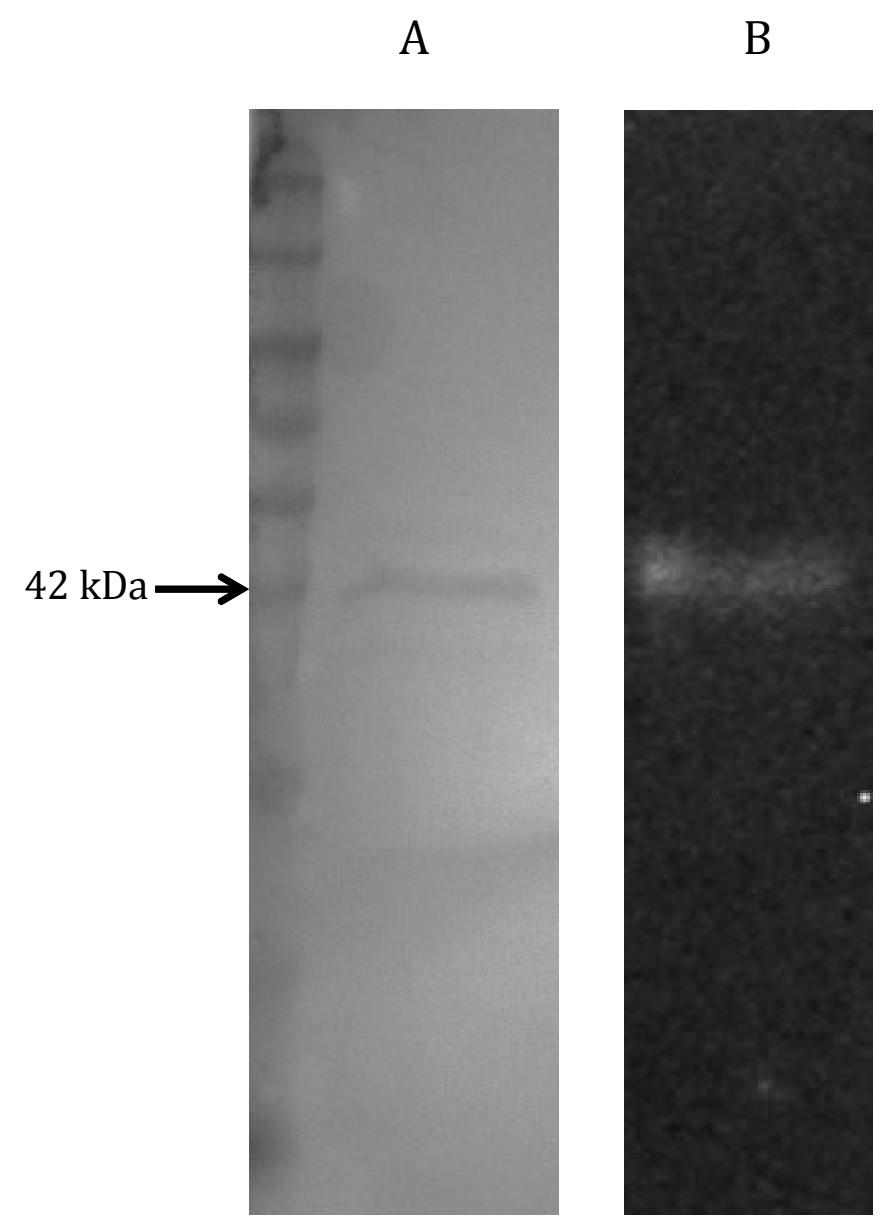

Figure 6.1 Purified GSK3 from the skeletal muscle of ACR 13-lined ground squirrels. (A) Coomassie Brilliant blue stained PVDF membrane, and (B) Western blot using an anti-GSK $3 \beta$ antibody. The arrow indicates the $42 \mathrm{kDa}$ band on the protein ladder. 
A

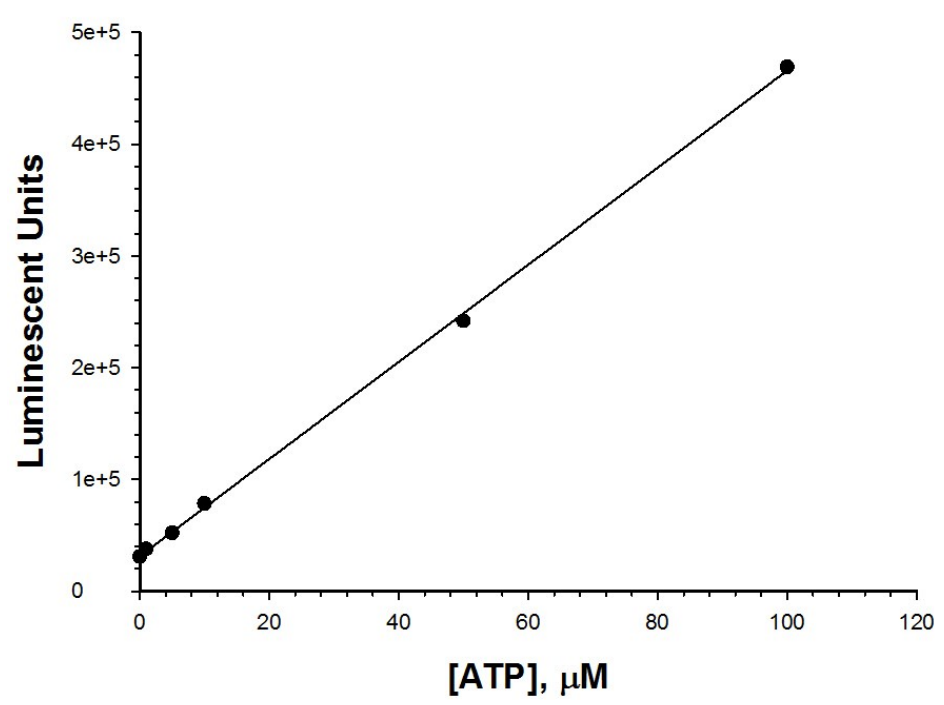

B

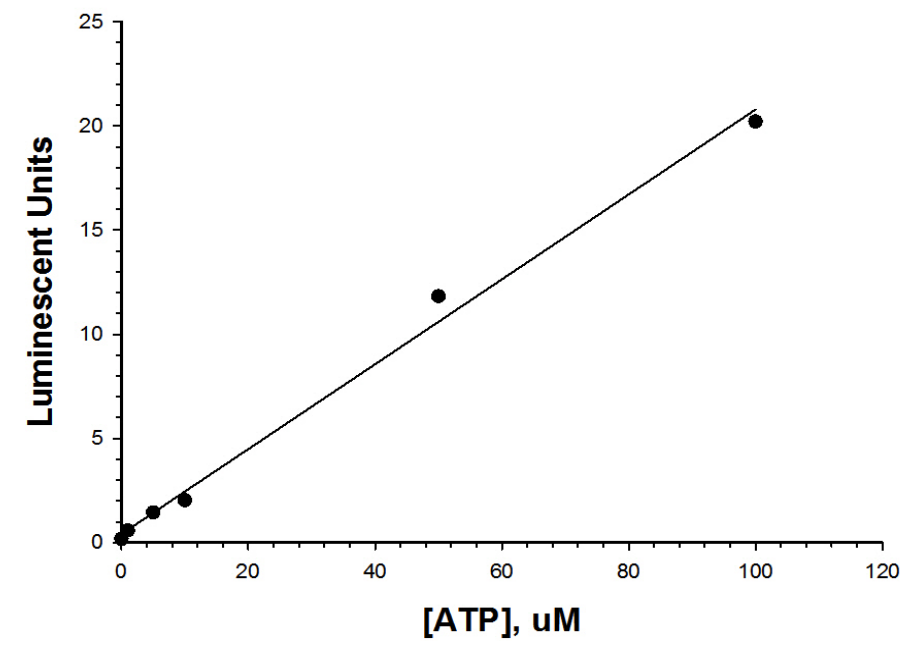

Figure 6.2 ATP standard curves for Kinase-Glo Plus luminescent kinase assay platform.

(A) The ATP standard curve when utilizing the Chemigenius Bioimaging System, and (B) the ATP standard curve when utilizing the Cary Eclipse fluorescent spectrophotometer. Data are means $\pm \mathrm{SEM}, \mathrm{n}=3$. 
$\mathbf{A}$

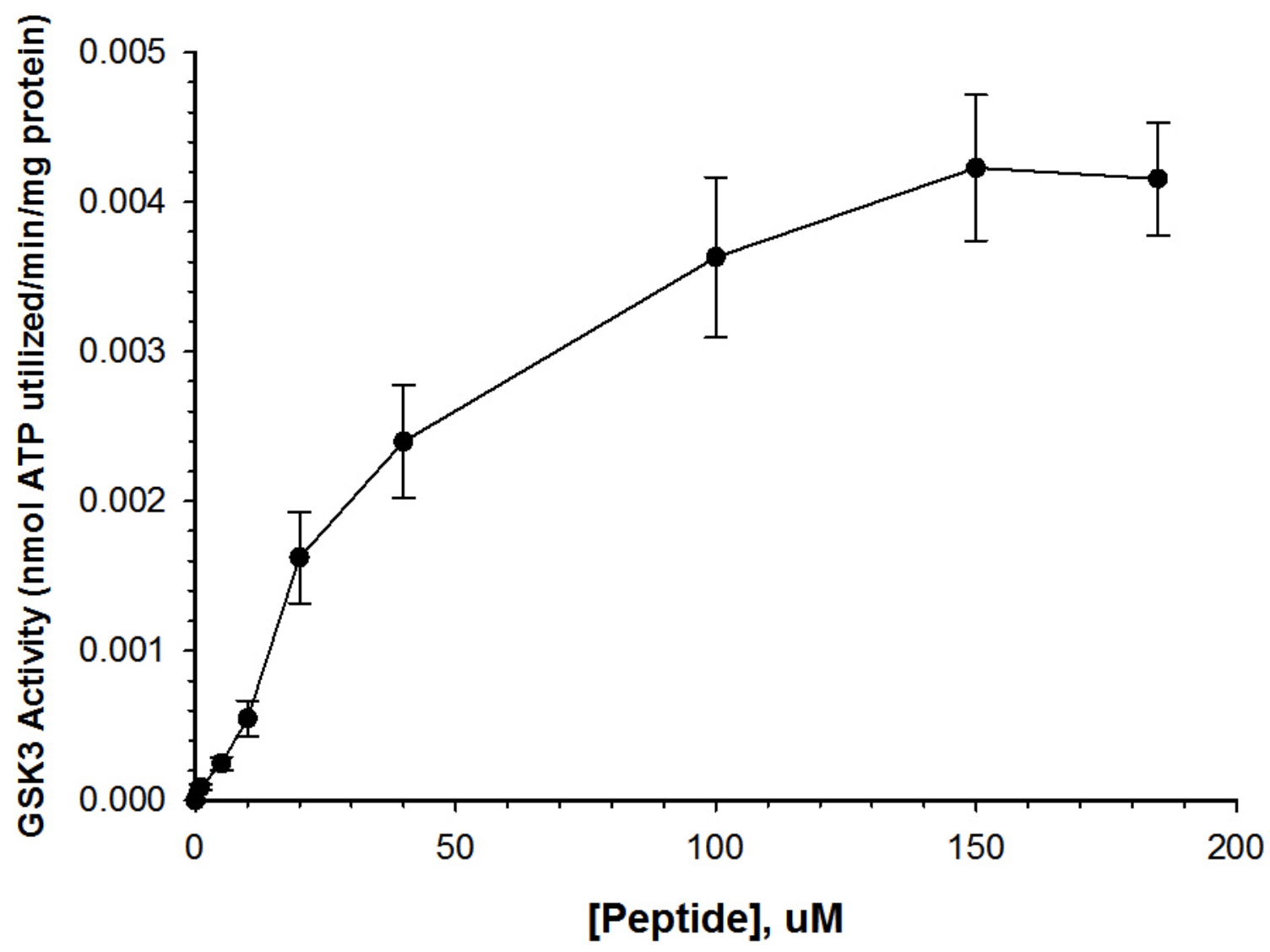

B

$\begin{array}{lllllllll}0 & 1 & 5 & 10 & 20 & 40 & 100 & 150 & 185\end{array}$

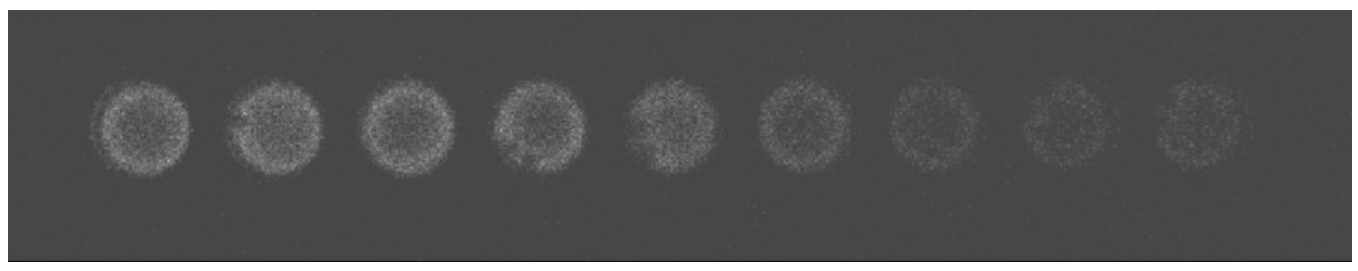

Figure 6.3 $\mathrm{K}_{\mathrm{m}}$ peptide for GSK3 derived from the skeletal muscle of 13-lined ground squirrels. (A) The kinetic curve for the determination of $\mathrm{K}_{\mathrm{m}}$ peptide at room temperature and (B) A representative sequence of luminescent wells that were used to generate kinetic curves shown in (A). The peptide concentrations are indicated above each luminescent well. The data representative in graph are means $\pm S E M, n=3$ on independent determinations on separate enzyme samples. 


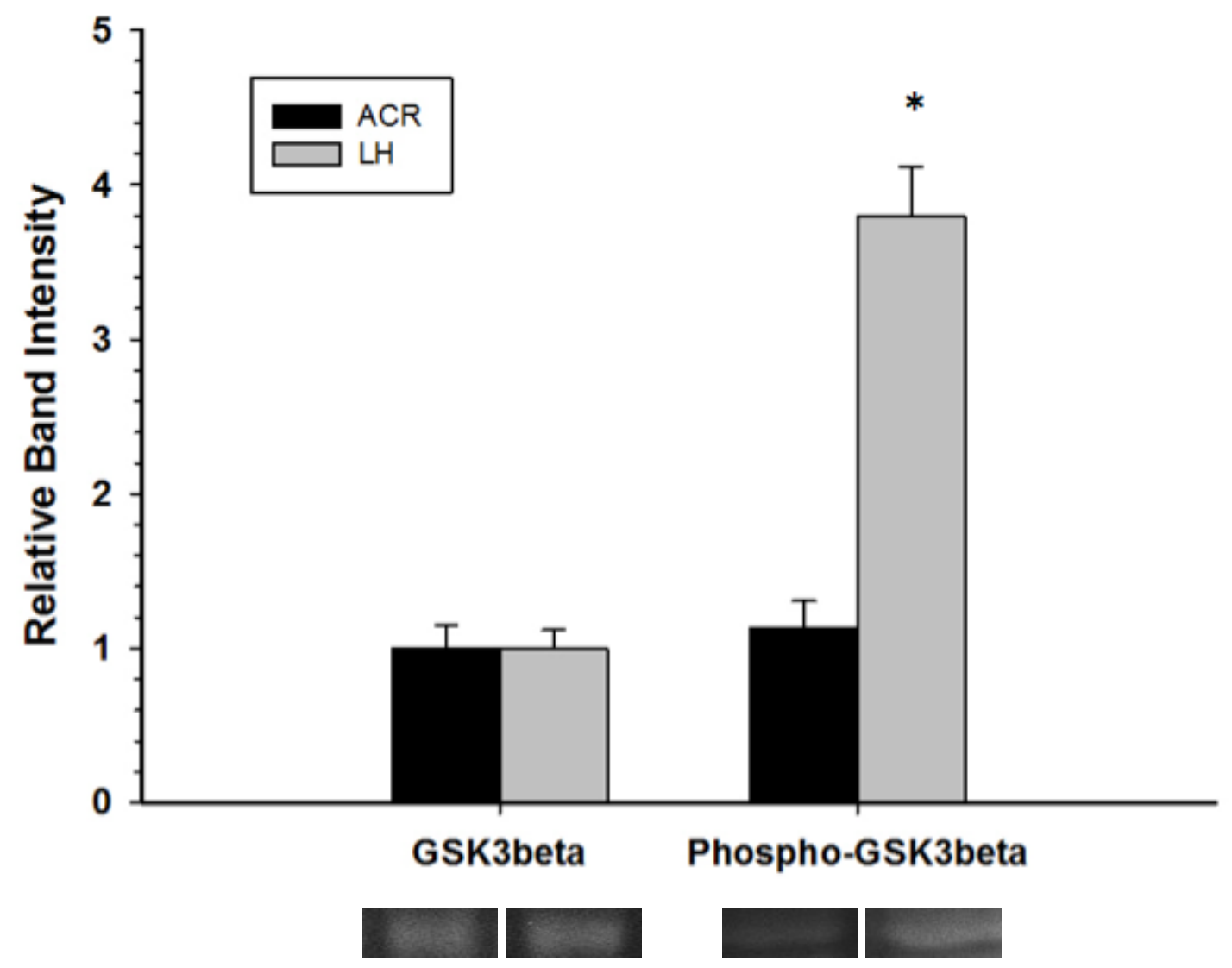

Figure 6.4 GSK3 $\beta$ and phospho-GSK3 $3($ Ser9) expression in crude 13-lined ground squirrel skeletal muscle samples from active (ACR) versus torpid (LH) animals. Data are means $\pm \mathrm{SEM}, \mathrm{n} \geq 5$ determinations on independently prepared samples. * -

Significantly different from the corresponding ACR value for the same condition using the Student's t-test, $\mathrm{p}<0.05$. 


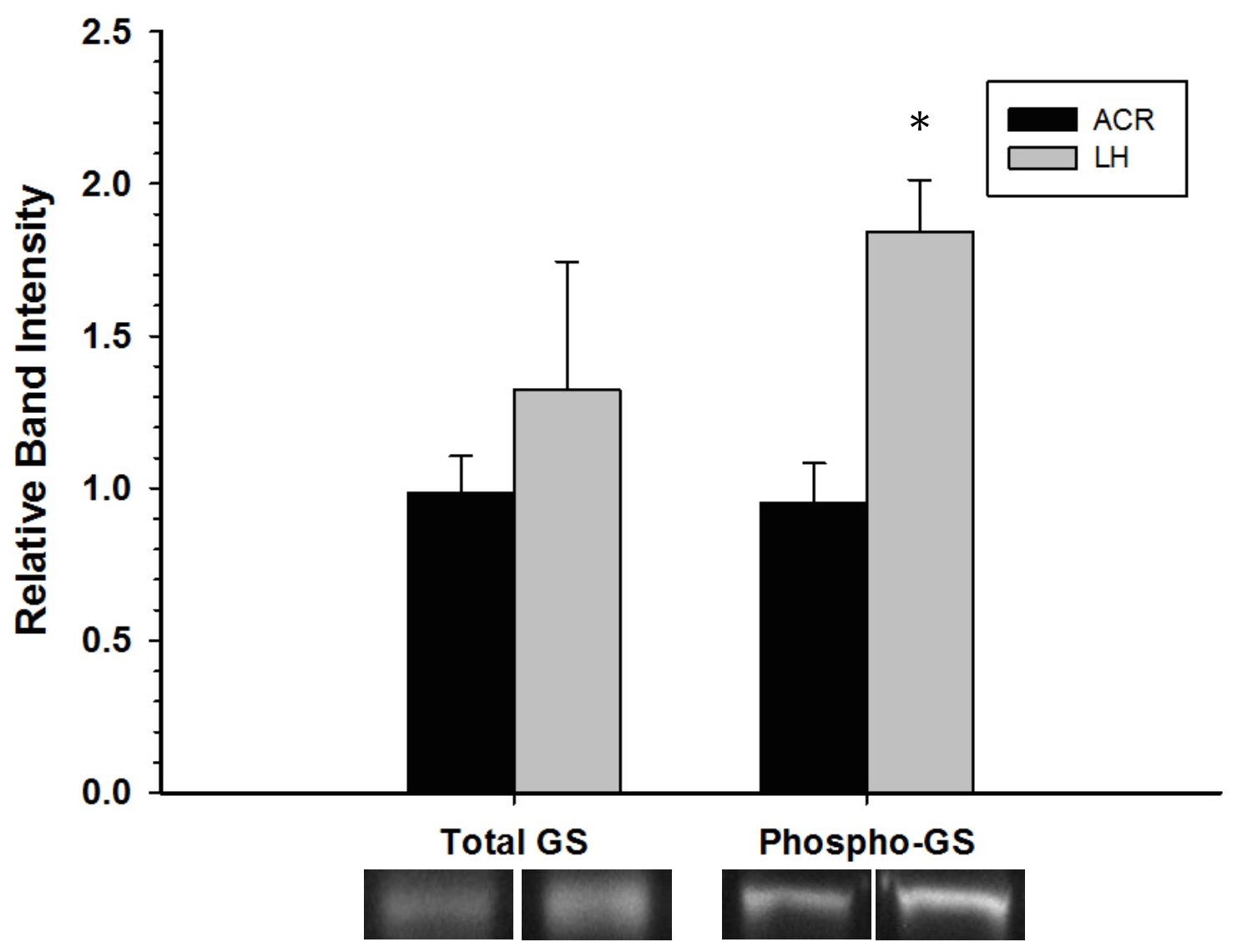

Figure 6.5 The levels of total GS and phospho(ser641/645)-GS expression in crude 13lined ground squirrel skeletal muscle samples from active (ACR) versus torpid (LH) animals. Data are means $\pm \mathrm{SEM}, \mathrm{n} \geq 3$ determinations on independently prepared samples. *- Significantly different from the corresponding value for the ACR condition using the Student's t-test, $\mathrm{p}<0.05$. 


\section{Chapter 7}

\section{General Discussion}


Harsh climates during the winter months in Canada and the northern United States present a significant obstacle to the survival of many organisms. The seasonal changes in temperature force an organism to either subsist on resources that remain available, migrate to warmer climates, or enter into a dormant state and emerge when more favorable conditions arise. Although it is the most complex strategy to employ, the latter approach is the one adopted by the Richardson's and thirteen-lined ground squirrel species for winter survival. These ground squirrels utilize numerous bouts of aerobic torpor during the hibernation season and subsist on endogenous fuel reserves until environmental conditions improve. While a complex array of biochemical and physiological changes occur within the ground squirrel to facilitate and maintain the torpid state, the careful regulation of energy production and usage may be the most critical aspect for this survival strategy. This is especially true for those hibernating species that do not forage during interbout arousals (which includes ground squirrels), but rely solely on the fixed fuel reserves compiled during pre-hibernation hyperphagia (cited in Humphries et al., 2003).

Torpor is a time when most tissues display a dramatic shift in the preferred fuel for energy production. Most tissues switch from carbohydrate to fatty acid oxidation for the majority of their energy needs. This is not to say that carbohydrate metabolism is unimportant during hibernation. Indeed, glucose catabolism and anabolism appears to fluctuate greatly depending of the stage of the hibernation cycle, and this suggests careful regulation of glycolysis and gluconeogenesis throughout hibernation. The present thesis aimed at assessing potentially important control points within carbohydrate catabolic and 
anabolic pathways, as well as investigating upstream regulators that may be important in controlling glucose metabolism during ground squirrel torpor.

With respect to skeletal muscle glucose catabolism, it is important to realize that the oxidation of glucose for energy occurs throughout the hibernation cycle. This is in contrast to the paradigm that during torpor, fatty acid oxidation is the sole source of energy for the dormant ground squirrel. Indeed, during torpor glucose consumption has been reported within skeletal muscle and, therefore, must contribute to the metabolic energy production required by the hibernators (Tashima et al., 1970). Conversion of total glucose utilized into the $\mathrm{CO}_{2}$ and ATP outputs that would be produced under aerobic conditions indicates that glucose may account for up to $10 \%$ of metabolic energy requirements during ground squirrel torpor (Morrison, 1960; Galster and Morrison, 1970). Moreover, the fact that glycolysis is still occurring during torpor is further corroborated by the continued production of pyruvate and lactate, albeit at a reduced rate (Galster and Morrison, 1970, Galster and Morrison, 1975; Hachimi et al., 1990). The contribution of carbohydrates is reported to increase steadily as the animal rewarms during interbout arousals (i.e. respiratory quotient nears 1), with glucose oxidation helping to provide energy for shivering thermogenesis (Lyman and Leduc, 1953; Buck and Barnes, 2000; Karpovich et al., 2009). Indeed, studies on fuel metabolism patterns during shivering thermogenesis in rats indicate that carbohydrate oxidation is dominant during intense shivering, which is likely similar to that seen during early ground squirrel rewarming (cited in Vaillencourt et al., 2009). The fact that these fluctuations occur repeatedly through the hibernation season and that a continual cyclic synthesis and degradation of glycolytic enzymes would be energetically uneconomical, it is reasonable 
to hypothesize that glycolysis would be regulated at the enzyme level by control mechanisms that can be quickly and easily applied. The main possibilities are covalent modification of enzymes such as by reversible phosphorylation and the actions of allosteric modifiers. Within ground squirrel skeletal muscle, regulation by posttranslational modification has already been demonstrated for the first enzyme of the glycolytic pathway, hexokinase, where reversible phosphorylation mediated the suppression of its activity during torpor (Abnous and Storey, 2008). The present studies in this thesis found that coordinated suppression of enzyme activity within the glycolytic pathway was not relegated to just hexokinase, but includes GAPDH and PK. The mechanism through which the latter enzyme was suppressed appears to be reversible phosphorylation, with increased PK phosphorylation during torpor leading to decreased activity. The mechanism by which GAPDH is regulated, on the other hand, was somewhat less clear. The evidence suggested that GAPDH suppression may be due to reversible tyrosine phosphorylation, however, the data obtained through mass spectrometry indicated that reversible acetylation or methylation could also be involved. Regardless, investigation into the kinetic action of skeletal muscle GAPDH and PK corroborates the results of the hexokinase study and indicates that glycolytic rate is slowed within skeletal muscle during torpor, and that the decrease is likely mediated through posttranslational modification of several glycolytic enzymes.

It has been the long-standing view that a single rate-limiting enzyme, typically at the beginning of a pathway, catalyzes a non-equilibrium reaction that controls flux through a pathway. Further, alterations in the activity of this enzyme, brought about by small molecular weight molecules or covalent modification, are thought to mediate flux 
through that pathway with subsequent enzymes being of sufficiently high activity to process metabolites without altering the pace set by the rate-limiting step. While this may be true in some cases, it is becoming increasingly evident that flux through many pathways is often dependent on regulation of multiple enzymes, and that the distribution of this control varies depending on the physiological state (Thomas and Fell, 1998). This appears to be especially true for the glycolytic pathway where numerous studies have indicated several points of control. For instance, studies in a variety of tissues within anoxia-tolerant turtles indicated that glycogen phosphorylase, phosphofructokinase (PFK), and PK all showed altered activity states during anoxia as compared to the enzymes from aerobic animals (Brooks and Storey, 1989). Similarly, Cowan et al. (2000) found that four glycolytic enzymes (PFK, GAPDH, aldolase, and PK) increased in activity in the spadefoot toad, Scaphiopus couchi, skeletal muscle during estivation. The points of regulation identified in these studies included both equilibrium and nonequilibrium reactions, which are important to note when attempting to assess flow through a particular pathway. Thus, the present study's assessment of GAPDH and PK help to give a more complete picture of the regulation and flow through the glycolytic pathway within ground squirrel skeletal muscle. Moreover, suppression of these two enzymes coincides well with the previous investigations of ground squirrel skeletal muscle hexokinase (Abnous and Storey, 2008) and PFK (MacDonald and Storey, 2005), thereby giving a clearer picture as to how glycolysis is regulated during torpor.

While glycolysis is generally thought to be an important pathway in virtually all cells, gluconeogenesis is often taken as irrelevant outside of the liver in mammalian systems. However, this is likely an oversimplification. It is true that gluconeogenesis is 
mainly confined to hepatic tissue, but the specific enzymes that are crucial for gluconeogenesis to occur (i.e. FBPase, phosphoenolpyruvate carboxykinase, and malic enzyme) are found in skeletal muscle of a number of vertebrate species, including several mammalian species (Opie and Newsholme, 1967). Moreover, various studies have shown that frog, rat, rabbit and human muscle have the ability to synthesize glucose under various conditions (Bendall and Taylor, 1970; Hermansen and Vaage, 1977; Fell et al., 1979; Fournier and Guderley, 1992). With respect to the ground squirrel, the role of gluconeogenesis in hibernation has seldom been studied. At most, the levels of muscle glycogen have been assessed throughout the hibernation cycle with varying results. Several studies indicate that muscle glycogen increases or is maintained during torpor (Hannon and Vaughn, 1961; el Hachimi et al., 1990; James et al., 2013), while others indicate a net decrease in glycogen during dormancy (Zimny and Tyrone, 1957; Galster and Morrison, 1970; Galster and Morrison, 1975). This contradiction may be explained by examining the timing of tissue sampling within the torpor bout. Early studies by Zimny and Tyrone (1957) and Hannon and Vaughn (1961) sacrificed animals anywhere from 3-5 days after entrance into torpor whereas Galster and Morrison (1970; 1975) determined glycogen content at various times from 2-12 days after torpor initiation. The latter studies likely depict the most accurate state of muscle glycogen content during a hibernation cycle due to its multiple time points. These studies showed that upon transition from late arousal to early torpor there is a significant increase in glycogen content within ground squirrel muscle. However, as torpor continues glycogen content decreases until the next interbout arousal period. Thus, it appears that glycolysis dominates over gluconeogenesis for the majority of the torpor/arousal cycle. 
Given that gluconeogenic rates are likely reduced during torpor but may increase during late arousal, two things were hypothesized: (1) gluconeogenic enzymes are suppressed during torpor, and (2) gluconeogenic enzymes are regulated at the enzyme level so as to easily accommodate increased/decreased flux depending on the stage of the torpor/arousal cycle. This may indeed be the case for skeletal muscle GAPDH, which showed decreased activity in the gluconeogenic direction as well as possible regulation by reversible tyrosine phosphorylation, acetylation or methylation (Chapter 2). Similarly, skeletal muscle FBPase activity was suppressed during torpor, with reversible phosphorylation being the underlying mechanism (Chapter 4). Thus, it appears that there is a coordinated suppression of gluconeogenic enzymes during torpor by reversible mechanisms that can be quickly reversed during interbout arousals when glucose synthesis is likely reestablished.

An unexpected and yet interesting aspect of this thesis is the prevalence of posttranslational acetylation on glycolytic and gluconeogenic enzymes. If the potential site-specific increase in GAPDH acetylation of the enzyme from torpid animals is included, all three metabolic enzymes investigated in this study displayed increased protein acetylation during dormancy. Indeed, reversible acetylation is becoming a burgeoning field of cellular metabolic regulation, with numerous examples of acetylation regulating carbohydrate anabolism and catabolism. For instance, reversible acetylation has recently been shown to be an important aspect of prokaryotic and eukaryotic regulation of metabolism, with most glycolytic and gluconeogenic enzymes showing evidence of acetylation (Zhao et al., 2010; Wang et al., 2011; Xiong et al., 2011). Interestingly, reversible acetylation of glycolytic and gluconeogenic enzymes was 
dependent on the availability of carbohydrates for energy. Moreover, enzymes important to fatty-acid oxidation in fasting mice demonstrate a significant reduction in protein acetylation, which could suggest that protein acetylation may be important to the torpid/fasting ground squirrel (Hirschey et al., 2010). Acetylation may well rival phosphorylation as a key regulator of carbohydrate metabolism, as well as many other processes within the cell. Future studies need to be focused on determining the role of posttranslational acetylation on the function of proteins, such as those investigated here. Furthermore, the upstream acetylases and deacetylases need to be investigated to determine the potentially important regulators of non-histone protein acetylation. Although reversible acetylation is becoming a highly prevalent posttranslational modification for many cells, reversible phosphorylation remains one of the most important regulatory mechanisms for the cell. This thesis investigated two key regulators of protein phosphorylation: GSK3 and PP2A. The former was found to be less active during torpor with site-specific reversible phosphorylation being the most likely underlying mechanism of GSK3 suppression. The activity state of GSK3 is important to consider when assessing the regulation of carbohydrate metabolism since it typically controls one of the key enzymes involved in synthesizing glycogen - glycogen synthase (Fig. 7.1). In apparent contradiction to the decrease in GSK3 activity, glycogen synthase was found to be significantly more phosphorylated at two key residues - Ser641 and Ser645 - during torpor as compared to the enzyme from the euthermic condition (Chapter 6). This suggests that glycogen synthase is less active during torpor as the aforementioned residues are closely associated with enzyme activity (Picton et al., 1982; DePaoli-Roach et al., 1983; Fiol et al., 1987). This result coincides with the results for 
skeletal muscle GAPDH (Chapter 3) and FBPase (Chapter 5), which indicate a reduction in enzyme activity in the gluconeogenic direction. Taken together this is sound evidence for an overall suppression of glucose and glycogen synthesis during ground squirrel torpor.

Another point of interest is the potential regulation of skeletal muscle FBPase by GSK3-dependent phosphorylation. Recent evidence has suggested that GSK3 may regulate the translocation of skeletal muscle FBPase into the nucleus, where it mediates important cellular events related to cell survival (Gizak et al., 2012). Nuclear translocation of FBPase was not investigated in this study but would be an interesting topic for a future project.

Equally important to protein phosphorylation in regulating enzyme activity is protein dephosphorylation brought about by protein phosphatases. This thesis investigated the activity and regulatory properties of PP2A, which is well known to exert effects at numerous points within carbohydrate anabolic and catabolic processes. These effects are mainly shown in vitro and in a wide range of organisms, including regulation of GSK3 in mammalian cell lines (Mora et al., 2002; Hernandez et al., 2010; Qian et al., 2010), dephosphorylation of hexokinase in the freeze tolerant wood frog (Dieni and Storey, 2011), and the dephosphorylation of GAPDH, fructose bisphosphate aldolase, and pyruvate kinase in yeast (Bernal et al., 2014). PP2A is also integral in responding to increases in cellular glucose by dephosphorylating and activating the carbohydrate responsive element binding protein (ChREBP) transcription factor (Kawaguchi et al., 2001). For these reasons PP2A was chosen as a potentially important factor in carbohydrate metabolism during ground squirrel torpor. Results from this study indicate 
that PP2Ac was suppressed during torpor, which was correlated with a significant increase in Y307 phosphorylation of the catalytic subunit. Computational analysis of the effects of Y307 phosphorylation indicated that this posttranslational modification causes a decrease in PP2Ac affinity for its protein substrate. Given that PK and GSK3 are known substrates for PP2A (Mora et al., 2002; Klumpp and Krieglstein, 2003; Hernandez et al., 2010; Qian et al., 2010) (Fig. 7.1), their elevated phosphorylation states found in this study may be made possible by the stable suppression of PP2Ac. Thus, PP2A suppression may play a critical role in maintaining the state of skeletal muscle carbohydrate metabolism during ground squirrel torpor.

The prevalence of numerous posttranslational modifications on the proteins investigated in this thesis is of particular importance as it suggests that regulation of protein function is far more complicated than simply one modification - one effect dynamics. Crosstalk between posttranslational modifications is actually quite common with some initial modifications preventing additional moieties from being added onto the protein, and some modifications stimulating further alterations (Yang and Seto, 2008; van Noort et al., 2012). An example of the former involves the acetylation of two key sites on MAPKK within the MAPK cascade, which blocks upstream phosphorylation by MAPKKK (Mukherjee et al., 2006). Alternatively, an example of positive crosstalk, which is related to a chapter in this thesis, involves the priming phosphorylation event that precedes the GSK3-mediated phosphorylation of glycogen synthase. Phosphorylation commonly plays this role and tends to seed further posttranslational modifications, such as the phosphorylation dependent ubiquitination of an E3 ligase substrate, or the phosphorylation dependent SUMOylation of heat shock factor protein 1 
(HSF1; reviewed in Hunter, 2007). While these examples illustrate the complexity of posttranslational regulation of enzymes, it is still unknown how the multitude of potential modifications can affect GAPDH, PK, FBPase and PP2Ac function in ground squirrels organs during hibernation. Future studies should investigate the interplay of the posttranslational modifications identified here and the effect these modifications on enzyme structure and function.

While not the focus of this thesis, the regulatory landscape regarding ground squirrel skeletal muscle carbohydrate metabolism would not be complete without the consideration of allosteric modifiers. For instance, some of the more important allosteric modifiers of metabolic enzymes are the adenylates (AMP, ADP, and ATP). Indeed, skeletal muscle PK is inhibited by cellular ATP (Tanaka et al., 1967), and in this study, was shown to be significantly less sensitive to ATP inhibition during torpor as compared to the enzyme from the euthermic animal (Chapter 3). Furthermore, FBPase is widely known to be inhibited by cellular AMP, and in this study, was shown to be significantly less susceptible to AMP-dependent inhibition during torpor as compared to the enzyme from the euthermic animal (Chapter 4). In both of the aforementioned cases, response to adenylates suggests a higher activity state for PK and FBPase during torpor. With respect to PK, intracellular ATP concentrations are reported to be near the measured $\mathrm{I}_{50}$ ATP for enzymes derived from both euthermic and torpid conditions, and thus may represent a significant modifier of PK activity in vivo. That being said, the 2.5 -fold increase in $\mathrm{K}_{\mathrm{m}}$ PEP during torpor is likely the most dominant determinant of PK activity in vivo, effectively suppressing PK activity during this period. With respect to FBPase, skeletal muscle concentrations of AMP likely exceed $\mathrm{I}_{50}$ AMP measured in this study for 
the enzyme derived from either condition (English and Storey, 2000). This would suggest that FBPase would be substantially, if not completely, inhibited at all times throughout a hibernation season. While this is unlikely to be the case, AMP may be a significant factor in controlling FBPase activity.

In addition to the adenylate molecules, many metabolic enzymes are affected by small metabolites. For instance, FBPase is inhibited by F2,6 2 , and this inhibition would appear to be complete at the levels of this molecule typically present in mammalian muscle tissue. Similar to the situation with AMP, complete inhibition of FBPase is unlikely and it is far more likely that the free $\mathrm{F} 2,6 \mathrm{P}_{2}$ in vivo is significantly less than measured concentration in vitro. That being said, this small molecular weight metabolite cannot be ruled out as an important regulator of ground squirrel skeletal muscle FBPase during torpor.

\section{Conclusion}

The regulation of energy production and expenditure is of the upmost importance during mammalian hibernation, especially for those hibernators that survive on fixed internal reserves of fuels for their entire hibernation season. Pre-hibernation hyperphagia, that is typical for many hibernators including the ground squirrel, sets the stage for the metabolic landscape during hibernation by specifically building up energy rich stores of fat for the switch to a lipid-based economy during torpor in most tissues. This fact suggests a de-emphasis on carbohydrate metabolism during torpor, and for the skeletal muscle the suppression of GAPDH, PK and FBPase bears this out. Each of the aforementioned enzymes appears to be reversibly modified so as to mediate their suppression during torpor, which may be critical in allowing re-activation of these 
enzymes during interbout arousals, where carbohydrate catabolism and anabolism are thought to increase. Potentially important mediators of these and other changes in glycolytic and gluconeogenic enzyme function include protein kinases and protein phosphatases. The current study of GSK3 suggests that this kinase may not be a key modifier of metabolic enzymes during torpor since site-specific phosphorylation at Ser9 on GSK3 $\beta$ efficiently suppressed its activity. However, evidence gathered about PP2Ac suggests that although this phosphatase is less active during torpor, its suppression likely contributes substantially to skeletal muscle metabolic state by maintaining the inhibitory phosphorylation state of crucial glycolytic and gluconeogenic enzymes during torpor. Taken together the work presented in this thesis provides novel insights into the regulation of skeletal muscle carbohydrate metabolism during ground squirrel torpor. 


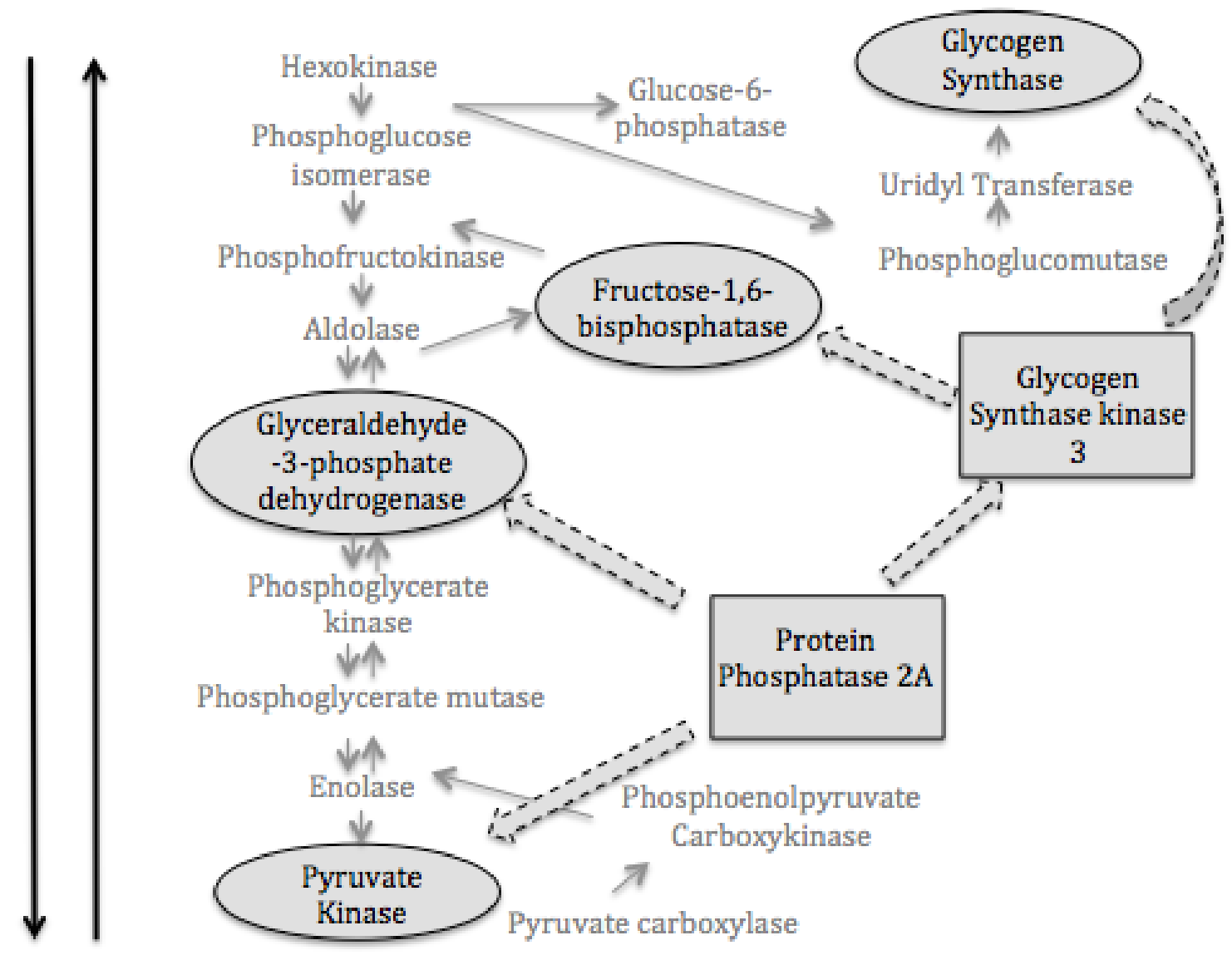

Figure 7.1. Enzymes of carbohydrate metabolism. The glycolytic pathway can be traced by following arrow (far left side) going from the top to the bottom and gluconeogenesis is the reverse. Enzymes that are circled and in bold are the enzymes that were investigated in this study. Dashed arrows indicate potential targets of either PP2A or GSK3 in the gluconeogenic or glycolytic pathways. 


\section{References}


Abnous, K., and Storey, K.B. (2008). Skeletal muscle hexokinase: regulation in mammalian hibernation. Mol. Cell. Biochem. 319: 41-50.

Alessi, D.R., Caudwell, F.B., Andjelkovic, M., Hemmings, B.A. and Cohen, P. (1996). Molecular basis for the substrate specificity of protein kinase B; comparison with MAPKAP kinase-1 and p70 S6 kinase. FEBS Letters 399: 333-338.

Alessi, D.R., James, S.R., Downes, C.P., Holmes, A.B., Gaffney, P.R.J., Reese, C.B., and Cohen, P. (1997). Characterization of a 3-phosphoinositide-dependent protein kinase which phosphorylates and activates protein kinase Ba. Curr. Biol. 7: 261269.

Almarza, J., Rincon, L., Bahsas, A., and Brito, F. (2009). Molecular mechanism for the denaturation of proteins by urea. Biochemistry 48(32): 7608-7613.

Baba, T., Kobayashi, H., Kawasaki, H., Mineki, R., Naito, H., and Ohmori, D. (2010). Glyceraldehyde-3-phosphate dehydrogenase interacts with phosphorylated Akt resulting from increased blood glucose in rat cardiac muscle. FEBS Lett. 584(13): 2796-2800.

Baibai, T., Oukhattar, L., Moutaouakkil, A., and Soukri, A. (2007).Purification and characterization of glyceraldehyde-3-phosphate dehydrogenase from European pilchard Sardina pilchardus. Acta Biochim. Biophys. Sin (Shanghai) 39(12): 947954.

Ballou, L.M., Tian, P.Y., Lin, H.Y., Jiang, Y.P., and Lin, R.Z. (2001). Dual regulation of glycogen synthase kinase- $3 \beta$ by the $\alpha 1 \mathrm{~A}$-adrenergic receptor. J. Biol. Chem. 276: 40910-40916.

Bauman, W.A., Meryn, S., and Florant, G.L. (1987). Pancreatic hormones in the nonhibernating and hibernating golden mantled ground squirrel. Comp. Biochem. Physiol. A Physiol. 86: 241-244.

Bendall, J.R. and Taylor, A.A. (1970). The Meyerhof quotient and the synthesis of glycogen from lactate in frog and rabbit muscle. Biochem. J. 118: 887-893.

Bernall, M., Zhurinsky, J., Iglesias, A.B., Snachez-Romero, M.A., Flor-Parra, I., TomasGallardo, L., Perez-Pulido, A.J., Jimenez, J., and Daga, R.R. (2014). Proteomewide search for PP2A substrates in fission yeast. Proteomics 14:1367-1380.

Bijlani, R.L., Bijlani, R.L., and Manjunatha, S. (2010). Membrane potential at rest and during activity, In: Understanding Medical Physiology: A Textbook for Medical Students. JP Medical Ltd. Pg. 118.

Bjellvqist, B., Hughes, G.J., Pasquali, C., Paquet, N., Ravier, F., Sanchez, J., Frutiger, S., and Hochstrasser, D.F. (1993). The focusing positions of polypeptides in immobilized $\mathrm{pH}$ gradients can be predicted from their amino acid sequences. Electrophoresis 14: 1023-1031.

Bjellqvist, B., Basse, B., Olsen, E., and Celis, J.E. (1994). Reference points for comparisons of two-dimensional maps of proteins from different human cell types 
defined in a $\mathrm{pH}$ scale where isoelectric points correlate with polypeptide compositions. Electrophoresis 15: 529-539.

Blom, N., Gammeltoft, S., and Brunak, S. (1999). Sequence- and structure-based prediction of eukaryotic protein phosphorylation sites. J. Mol. Biol. 294: 13511362.

Boivin, P., Galand, C., and Estrada, M. (1980). Phosphorylation of human red cell and liver pyruvate kinase. Differences between liver and erythrocyte L-type subunits. Cell. Mol. Life Sci. 36(8): 900-901.

Boulatnikov, I.G., Nadeau, O.W., Daniels, P.J., Sage, J.M., Jeyasingham, M.D., Villar, M.T., Artiques, A., and Carlson, G.M. (2008). The regulatory beta subunit of phosphorylase kinase interacts with glyceraldehyde-3-phosphate dehydrogenase. Biochemistry 47: 7228-7236.

Bouma, H.R., Carey, H.V., and Kroese, G.M. (2010). Hibernation: the immune system at rest? J. Leukocyte Biol. 88: 619-624.

Brooks, S.P.J. (1992). A simple computer program with statistical tests for the analysis of enzyme kinetics. Biotechniques 13: 906-911.

Brooks, S.P.J. (1994). A program for analyzing enzyme rate data obtained from a microplate reader. Biotechniques. 17: 1155-1161.

Brooks, S.P.J. and Storey, K.B. (1989). Regulation of glycolytic enzymes during anoxia in the turtle Pseudemys scripta. Amer. J. Physiol. 257: R278-R283.

Brooks, S.P.J. and Storey, K.B. (1992). Mechanism of glycolytic control during hibernation in the ground squirrel, Spermophilus lateralis. J. Comp. Physiol. A 118: 1103-1114.

Buck, C.L. and Barnes, B.M. (2000). Effects of ambient temperature on metabolic rate, respiratory quotient, and torpor in an arctic hibernator. Am. J. Physiol. 279: R255-R262.

Burlington, R.F. and Klain, G.J. (1967). Gluconeogenesis during hibernation and arousal from hibernation. Comp. Biochem. Physiol. 22: 701-708.

Cai, D., McCarron, R.M. Yu, E.Z., Li, Y., and Hallenbeck, J. (2004). Akt phosphorylation and kinase activity are down-regulated during hibernation in the 13-lined ground squirrel. Brain Res. 1014: 14-21.

Carey, H.V., Andrews, M.T., and Martin, S.L. (2003). Mammalian hibernation: Cellular and molecular responses to depressed metabolism and low temperature. Physiol. Rev. 83: 1153-1181.

Chakrabarti, P., English, T., Shi, J., Smas, C.M. and Kandor, K.V. (2010). Mammalian target of rapamycin complex 1 suppresses lipolysis, stimulates lipogenesis and promotes fat storage. Diabetes 59: 775-781.

Chen, J., Martin, B.L., and Brautigan, D.L. (1992). Regulation of protein serine-threonine phosphatase type-2A by tyrosine phosphorylation. Science 257: 1261-1264. 
Choudhary, S., De, B.P., and Banerjee, A.K. (2000). Specific phosphorylated forms of glyceraldehydes-3-phosphate dehydrogenase associate with human parainfluenza virus type 3 and inhibit viral transcription in vitro. J. Virol. 74(8): 3634-3641.

Choudhary, C., Kumar, C., Gnad, F., Nielsen, M.L., Rehman, M., Walther, T.C., Olsen, J.V., and Mann, M. (2009). Lysine acetylation targets protein complexes and coregulates major cellular functions. Science 325: 834-840.

Cohen, P. (1985). The role of protein phosphorylation in the hormonal control of enzyme activity. Eur. J. Biochem. 151: 439-448.

Cornell, W.D. Cieplak, P., Bayly, C.I., Gould, I.R., Merz Jr., K.M., Ferguson, D.M., Spellmeyer, D.C., Fox, T., Caldwell, J.W., and Kollman, P.A. (1995). A second generation force field for the simulation of proteins, nucleic acids, and organic molecules. J. Am. Chem. Soc. 117: 5179-5197.

Cowan, K.J. and Storey, K.B. (1999). Reversible phosphorylation of skeletal muscle pyruvate kinase and phosphofructokinase during estivation in the spadefoot toad, Scaphiopus couchii. Mol. Cell. Biochem. 195: 173-181.

Cowan, K.J., MacDonald, J.A., Storey, J.M., and Storey, K.B. (2000). Metabolic reorganization and signal transduction during estivation in the spadefoot toad. EBO 5: 61-85.

Cross, D.A., Alessi, D.R., Cohen, P., Andjelkovich, M., and Hemmings, B.A. (1995). Inhibition of glycogen synthase kinase-3 by insulin mediated by protein kinase B. Nature 378: 785-789.

Daan, S., Barnes, B.M., and Strijkstra, A.M. (1991). Warming up for sleep - ground squirrels sleep during arousals from hibernation. Neuroscience Lett. 128: 265-268.

Davis, D.E. (1976). Hibernation and circannual rhythms of food consumption in marmots and ground squirrels. Quart. Rev. Biol. 51: 477-514.

DePaoli-Roach, A.A., Ahmad, Z., Camici, M., Lawrence Jr., J.C., and Roach, P.J. (1983). Multiple phosphorylation of rabbit skeletal muscle glycogen synthase. Evidence for interactions among phosphorylation sites and the resolution of electrophoretically distinct forms of the subunit. J. Biol. Chem. 258: 1070210709.

Dhalluin, C., Carlson, J.E., Zeng, L., He, C., Aggarwai, A.K., Zhou, M.M. (1999). Structure and ligand of a histone acetyltransferase bromodomain. Nature 399(6735): 491-496.

Dieni, C.A., Bouffard, M.C., and Storey, K.B. (2012). Glycogen synthase kinase-3: cryoprotection and glycogen metabolism in the freeze-tolerant wood frog. J. Exp. Biol. 215: 543-551.

Dieni, C.A. and Storey, K.B. (2011). Regulation of hexokinase by reversible phosphorylation in skeletal muscle of a freeze-tolerant frog. Comp. Biochem. Physiol. B Biochem. Mol. Biol. 159: 236-243. 
Ding, Q., Xia, W., Yang, J.Y., Lee, D.F., Xia, J., Bartholomeusz, G., Li, Y., Pan, Y., Li, Z., Bargou, R.C., Qin, J., Lai, C.C., Tsai, F.J., and Hung, M.C. (2005). Erk associates with and primes GSK-3beta for its inactivation resulting in regulation of beta-catenin. Mol. Cell. 19: 159-170.

Doble, B.W., and Woodgett, J.R. (2003). GSK-3 tricks of the trade for a multi-tasking kinase. J. Cell Sci. 116: 1175-1186.

Dohm, G.L., Patel, V.K., and Kasperek, G.J. (1986). Regulation of muscle pyruvate metabolism during exercise. Biochem. Med. Metabol. Biol. 35(3): 260-266.

Donella-Deana, A., Boschetti, M., and Pinna, L.A. (2003). Dephosphorylation of phosphothreoine peptides In: Methods in Enzymology (ed. Klumpp, S., and Krieglstein, J.). Gulf Professional Publishing pg. 5-7.

Duvel, K., Yecies, J.L., Menon, S., Raman, P., Lipovsky, A.L., Triantafellow, E., Ma, Q., Gorski, R., Cleaver, S., Vander Heiden, M.G., MacKeigen, J.P., Finan, P.M., Clish, C.B., Murphy, L.O., and Manning, B.D. (2010). Activation of a metabolic gene regulatory network downstream of mTOR complex 1. Mol. Cell 39:171-183.

Dzugaj, A. and Kochman, M. (1980). Purification of human liver fructose-1,6bisphosphatase. Biochim. Biophys. Acta 614: 407-412.

Eddy, S.F. and Storey, K.B. (2003). Differential expression of Akt, PPAR $\gamma$, and PGC-1 during hibernating bats. Biochem. Cell Biol. 81: 269-274.

Eddy, S.F., Morin Jr., P., and Storey, K.B. (2005). Cloning and expression of PPAR $\gamma$ and PGC $-1 \alpha$ from the hibernating ground squirrel Spermophilus tridecemlineatus. Mol. Cell Biochem. 269: 175-182.

Eigenbrodt, E., Abdel-Fattah Mostafa, M., and Schoner, W. (1977). Inactivation of pyruvate kinase type $\mathrm{M} 2$ from chicken liver by phosphorylation catalyzed by a cAMP-independent protein kinase. Hoppe-Seyler's Z. Physiol. Chem. 358: 10471055.

Ekdahl, K. (1988). In vitro phosphorylation of fructose-1,6-bisphosphatase from rabbit and pig liver with cyclic AMP-dependent protein kinase. Arch. Biochem. Biophys. 262: 27-31.

Ekman, P., and Jagar, O. (1993). Quantification of subnanomolar amounts of phosphate bound to seryl and threonyl residues in phosphoproteins using alkaline hydrolysis and malachite green. Anal. Biochem. 214: 138-141.

Emmerson, L.E., Dahl, T.A., and Martin, S.L. (2004). Quantitative analysis of liver protein expression during hibernation in the golden-mantled ground squirrel. Mol. Cell. Proteomics 3: 920-933.

English, T.E. and Storey, K.B. (2000). Enzymes of adenylate metabolism and their role in hibernation of the white-tailed prairie dog, Cynomys leucurus. Arch. Biochem.

Biophys. 376(1): 91-100. 
Fang, X., Yu, S.X., Lu, Y., Bast Jr., R.C., Woodgett, J.R., and Mills, G.B. (2000). Phosphorylation and inactivation of glycogen synthase kinase 3 by protein kinase A. Proc. Natl. Acad. Sci. USA 97: 11960-11965.

Fang, X., Yu, S., Tanyi, J.L., Lu, Y., Woodgett, J.R., and Mills, G.B. (2002). Convergence of multiple signaling cascades at glycogen synthase kinase 3: Edg receptor-mediated phosphorylation and inactivation by lysophosphatidic acid through a protein kinase C-dependent intracellular pathway. Mol. Cell. Biol. 22: 2099-2110.

Favre, B., Zolnierowicz, S., Turowski, P., and Hemmings, B.A. (1984). The catalytic subunit of protein phosphatase $2 \mathrm{~A}$ is carboxyl-methylated in vivo. J. Biol. Chem. 269, 16811-16817.

Ferkey, D.M. and Kimelman, D. (2002). Glycogen synthase kinase-3 $\beta$ mutagenesis identifies a common binding domain for GBP and axin. J. Biol Chem. 277: $16147-16152$.

Fell, R.D., McLane, J.A., Winder, W.W. and Holloszy, J.O. (1979). Preferential resynthesis of muscle glycogen in fasting rats after exhausting exercise. Intreg. Comp. Physiol. 7: R238-R332.

Finkemeier, I., Laxa, M., Miguet, L., Howden, A.J.M., and Sweetlove, L.J. (2011). Proteins of diverse function and subcellular location are lysine acetylated in Arabidopsis. Plant Physiol. 155: 1779-1790.

Fiol, C.J., Mahrenholz, A.M., Wang, Y., Roeske, R.W., and Roach, P.J. (1987). Formation of protein kinase recognition sites by covalent modification of the substrate. J. Biol. Chem. 262: 14042-14048.

Fister, P. Eigenbrodt, E., Presek, P., Reinacher, M., and Schoner, W. (1983). Pyruvate kinase type M2 is phosphorylated in the intact chicken liver cell. Biochem. Biophys. Res. Commun. 115(2): 409-414.

Force, T. and Woodgett, J.R. (2009). Unique and overlapping functions of GSK-3 isoforms in cell differentiation and proliferation abd cardiovascular development. J. Biol. Chem. 284: 9643-9647.

Fournier, P.A., and Guderley, H. (1992). Metabolic fate of lactate after vigorous exercise in the leopard frog, Rana pipiens. Am. J. Physiol. 262: R245-R254.

Fourrat, L., Iddar, A., and Soukri, A. (2007). Purification and characterization of cytosolic glyceraldehyde-3-phosphate dehydrogenase from the dromedary camel. Acta Biochim. Biophys. Sin (Shanghai) 39(2): 148-154.

Frame, S., Cohen, P., and Biondi, R.M. (2001). A common phosphate binding site explains the unique substrate specificity of GSK3 and its inactivation by phosphorylation. Mol.Cell 7: 1321-1327.

Frank, C.L., Karpovich, S., and Barnes, B.M. (2008). Dietary fatty acid composition and the hibernation patterns in free-ranging arctic ground squirrels. Physiol. Biochem. Zool. 81(4): 486-495. 
Frey, P.A., and Hegeman, A.D. (2007). Phosphotransfer and nucleotidyltransfer, In: Enzymatic reaction mechanisms. Oxford University Press US, pg 495-496.

Galster, W.A. and Morrison, P. (1966). Seasonal changes in serum lipids and proteins in the 13-lined ground squirrel. Comp. Biochem. Physiol. 18: 489-501.

Galster, W.A. and Morrison, P. (1970). Cyclic changes in carbohydrate concentrations during hibernation in arctic ground squirrel. Am. J. Physiol. 218: 1128-1232.

Galster, W.A. and Morrison, P. (1975). Gluconeogenesis in arctic ground squirrels between periods of hibernation. Am. J. Physiol. 228: 325-330.

Gasteiger, E., Hoogland, C., Gattiker, A., Duvand, S., Wilkins, M.R., Appel, R.D., and Bairoch, A. (2005). Protein identification and analysis tools on the ExPASy Server, In: The Proteomics Protocols Handbook. John M. Walker (ed.) Humana Press. pp. 571-607.

Gehnrich, S.C. and Aprille, J.R. (1988). Hepatic gluconeogenesis and mitochondrial function during hibernation. Comp. Biochem. Physiol. 91: 11-16.

Geiser, F. (1988). Reduction of metabolism during hibernation and daily torpor in mammals and birds: temperature effect or physiological inhibition? J. Comp. Phys. B 158: 25-37.

Geiser, F. (2004). Metabolic rate and body temperature reduction during hibernation and daily torpor. Annu. Rev. Physiol. 66: 239-274.

Gizak, A., Pirog, M., and Rakus, D. (2012). Muscle FBPase binds to cardiomyocyte mitochondria under glycogen synthase kinase-3 inhibition or elevation of cellular $\mathrm{Ca}^{2+}$ level. FEBS Lett. 586: 13-19.

Green, C.J., Brosnan, J.T., Fuller, B.J., Lowry, M., Stubbs, M., and Ross, B.D. (1984). Effect of hibernation on liver and kidney metabolism in 13-lined ground squirrels. Comp. Biochem. Physiol. 798: 167-171.

Greene, R.F. and Pace, C.N. (1974). Urea and guanidine hydrochloride denaturation of ribonuclease, lysozyme, alpha-chymotrypsin, and beta-lactoglobulin. J. Biol. Chem. 249(17): 5388-5393.

Gu, T.L., Cherry, J., Tucker, M., Wu, J., Reeves, C., and Polakiewicz, R.D. (2010). Identification of activated Tnk1 kinase in Hodgkin's lymphoma. Leukemia 24: 861-865.

Guan, K. and Xiong, Y. (2011). Regulation of intermediary metabolism by protein acetylation. Trneds in Biochemical Sciences 36: 108-116.

Guarente, L. (2011). The logic of linking protein acetylation and metabolism. Cell Metab. 14: 151-153.

Guo, W., Huang, N., Cai, J., Xie, W., and Hamilton, J.A. (2006). Fatty acid transport and metabolism in HepG2 cells. Am. J. Physiol. Gastrointest. Liver Physiol. 290: G528-G534.

Hachimi, Z.E., Tijane, M., Boissonnet, G., Benjouad, A., Desmadril, M., and Yon, J.M. (1990). Regulation of the skeletal muscle metabolism during hibernation of 
Jaculus orientalis. Comp. Biochem. Physiol. B 96(3): 457-459.

Hall, E.R., and Cottam, G.L. (1978). Isozymes of pyruvate kinase in vertebrates: Their physical, chemical, kinetic and immunological properties. Int. J. Biochem. 9: 785793.

Hanger, D.P., Hughes, J.R., Woodgett, J.R., Brion, J.P., and Anderton, B.H. (1992). Glycogen synthase kinase-3 induces Alzheimer's disease-like phosphorylation of tau: generation of paired helical filament epitopes and neuronal localization of the kinase. Neuroscience Lett. 147: 58-62.

Hannon, J.P., and Vaughn, D.A. (1961). Initial stages of intermediary glucose catabolism in the hibernator and nonhibernator. Am. J. Physiol. 201: 217-223.

Harlow, H.J. and Frank, C.L. (2001). The role of dietary fatty acids in the evolution of spontaneous and facultative hibernation patterns in prairie dogs. J. Comp. Physiol. B 171(1): 77-84.

Harwood, H.J., and Geyer, R.P. (1964). Biology Data book. Federation of American Societies for Experimental Biology, Washington, D.C.

Heldmaier, G., Klingenspor, M., Werneyer, M., Lampi, B.J., Brooks, S.P.J., Storey, K.B. (1999). Metabolic adjustments during daily torpor in the Djungarian hamster. Am. J. Physiol. Endocrinol. Metab. 276: E896-E906.

Hemmings, B.A., Yellowlees, D., Kernohan, J.C., and Cohen, P. (1981). Purification of glycogen synthase kinase 3 from rabbit skeletal muscle: Copurification with the activating factor $(\mathrm{Fa})$ of the (Mg-ATP) dependent protein phosphatase. Eur. J. Biochem. 119: 443-451.

Hermansen, L. and Vaage, O. (1977). Lactate disappearance and glycogen synthesis in human muscle after maximal exercise. Am. J. Physiol. 233: E422-E429.

Hernandez, F., Langa, E., Cuadros, R., Avila, J., and Villanueva, N. (2010). Regulation of GSK3 isoforms by phosphatases PP1 and PP2A. Mol. Cell Biochem. 344: 211215.

Hirschey, M.D., Shimazu, T., Goetzman, E., Jing, E., Schwer, B., Lombard, D.B., Grueter, C.A., Harris, C., Biddinger, S., Ilkayeva, O.R., Stevens, R.D., Li, Y., Saha, A.K., Ruderman, N.B., Bain, J.R., Newgard, C.B., Farese, R.V. Jr., Alt, F.W., Kahn, C.R., and Verdin, E. (2010). SIRT3 regulates mitochondrial fattyacid oxidation by reversible enzyme acetylation. Nature 464: 121-125.

Hitosugi, T., Kang, S., Vander Heiden, M.G., Chung, T., Elf, S., Lythgoe, K., Dong, S., Lonial, S., Wang, X., Chen, G.Z., Xie, J., Gu, T., Polakiewicz, R.D., Roesel, J.L., Boggon, T.J., Khuri, F.R., Gilliland, D.G., Cantley, L.C., Kaufman, J., and Chen, J. (2009). Tyrosine phosphorylation inhibits PKM2 to promote the Warburg effect and tumor growth. Sci. Signal. 2(97): ra73

Hittel, D., and Storey, K.B. (2001). Differential expression of adipose- and heart-type fatty acid binding proteins in hibernating ground squirrels. Biochim. Biophys. Acta 1522: 238-243. 
Hochachka, P.W. and Guppy, M. (1987). Metabolic arrest and the control of biological time. Harvard University Press, Cambridge, Mass.

Holden, C.P. and Storey, K.B. (1998). Protein kinase A from bat skeletal muscle: A kinetic study of the enzyme from a hibernating mammal. Arch. Biochem. Biophys. 358: 243-250.

Huang, Q., Lan, F., Zheng, Z., Xie, F., Han, J., Dong, L., Xie, Y., and Zheng, F. (2011) AKT2 suppresses GAPDH mediated-apoptosis in ovarian cancer via phosphorylating GAPDH at threonine 237 and decreasing its nuclear translocation. J. Biol. Chem. 286: 42211-42220.

Humphries, M.M., Thomas, D.W., and Kramer, D.L. (2003). The role of energy availability in mammalian hibernation: a cost-benefit approach. Physiol Biochem. Zool. 76: 165-179.

Hunter, T. (2007). The age of crosstalk: phosphorylation, ubiquitination, and beyond. Mol. Cell 28: 730-738.

Huston, A.L., Haeggstrom, J.Z., and Feller, G. (2008). Cold adaptation of enzymes: structural kinetic and microcalorimetric characterizations of an aminopeptidase from the Arctic psychrophile Colwellia psycherrythraea and of human leukotriene A4 hydrolase. Biochim. Biophys. Acta 1784: 1865-1872.

Iddar, A., Campos, L.A., Sancho, J., Serrano, A., and Soukri, A. (2003). Different thermostability of skeletal muscle glyceraldehyde-3-phosphate dehydrogenase from hibernating and euthermic Jerboa (Jaculus orientalis). Acta Biochim. Biophys. Sinica 35: 891-896.

James, R.S, Staples, J. F., Brown, J.C.L., Tessier, S.T., and Storey, K.B. (2013). The effects of hibernation on the contractile and biochemical properties of skeletal muscles in the thirteen-lined ground squirrel, Ictidomys tridecemlineatus. J. Exp. Biol. doi:10.1242/jeb.080663

Janssens, V., and Goris, J. (2001). Protein phosphatase 2A: a highly regulated family of serine/threonine phosphatases implicated in cell growth and signalling. Biochem. $J .353,417-439$.

Jeong, J.W., Bae, M.K., Ahn, M.Y., Kim, S.H., Sohn, T.K., Bae, M.H., Yoo, M.A., Song, E.J., Lee, K.J., and Kim, K.W. (2002). Regulation and destabilization of HIF1alpha by ARD1-mediated acetylation. Cell 111(5): 709-720.

Jiang, W., Wang, S., Xiao, M., Lin, Y., Zhou, L., Lei, Q., Xiong, Y., Guan, K., and Zhao, S. (2011). Acetylation regulates gluconeogenesis by promoting PEPCK1 degradation via recruiting the UBR5 ubiquitin ligase. Mol. Cell 43: 33-44.

Jurica, M.S., Mesecar, A., Heath, P.J., Shi, W., Nowak, T., and Stoddard, B.L. (1998). The allosteric regulation of pyruvate kinase by fructose-1,6-bisphosphate. Structure 6(2): 195-210.

Karpovich, S.A., Tolen, O., Buck, C.L., and Barnes, B.M. (2009). Energetics of arousal episodes in hibernating arctic ground squirrels. J. Comp. Physiol. B 179: 691-700. 
Kawaguchi, T., Takenoshita, M., Kabashima, T., and Uyeda, K. (2001). Glucose and cAMP regulate the L-type pyruvate kinase gene by phosphorylation/ dephosphorylation of the carbohydrate response element binding protein. Proc. Natl. Acad. Sci. USA 98: 13710-13715.

Kawamoto, R.M. and Caswell, A.H. (1986). Autophosphorylation of glyceraldehyde phosphate dehydrogenase and phosphorylation of protein from skeletal muscle microsomes. Biochemistry 25(3): 657-661.

Khew-Goodall, Y., and Hemmings, B.A. (1988). Tissue-specific expression of mRNAs encoding $\alpha$ - and $\beta$-catalytic subunits of protein phosphatase 2 A. FEBS Lett. 238: 265-268.

Khew-Goodall, Y., Mayer, R.E., Maurer, F., Stone, S.R., and Hemmings, B.A. (1991). Structure and transcriptional regulation of protein phosphatase $2 \mathrm{~A}$ catalytic subunit genes. Biochemistry 30: 89-97.

Klain, G.J. and Whitten, B.K. (1968). Carbon dioxide fixation during hibernation and arousal from hibernation. Comp. Biochem. Physiol. 25(1): 363-366.

Klein, P.S. and Melton, D.A. (1996). A molecular mechanism for the effect of lithium on development. Proc. Natl. Acad. Sci. USA 93: 8455-8459.

Kouzarides, T. (2000). Acetylation: a regulatory modification to rival phosphorylation? EMBO 19(6): 1176-1179.

Krog, J. (1954). Storing of food items in the winter nest of the Alaskan ground squirrel, Citellus undulates. J. Mammalogy 35: 586.

Kronfeld-Schor, N., Richardson, C., Silvia, B.A., Kunz, T.H., and Widmaier, E.P. (2000). Dissociation of leptin secretion and adiposity during prehiberatory fattening in little brown bats. Am. J. Physiol. Regl. Integr. Comp. Physiol. 279: R1277-R1281.

Kuma, Y., Campbell, D.G., and Cuenda, A. (2004). Identification of glycogen synthase as a new substrate for stress-activated protein kinase $2 \mathrm{~b} / \mathrm{p} 38 \beta$. Biochem. J. 379: 133-139.

Laplante, M., abd Sabatini, D.M. (2010). mTORC1 activates SREBP-1c and uncouples lipogenesis from gluconeogenesis. Proc. Natl. Acad. Sci. USA 107: 3281-3282.

Larkin, J.E., Franken, P., and Heller, H.C. (2002). Loss of circadian organization of sleep and wakefulness during hibernation. Am. J. Physiol. 282: R1086-R1095.

Larson, T.M., Laughlin, L.T., Holden, H.M., Rayment, I., and Reed G.H. (1994). Structure of rabbit muscle pyruvate kinase complexed with $\mathrm{Mn}^{2+}, \mathrm{K}^{+}$, and pyruvate. Biochemistry 33(20): 6301-6309.

Lechward, K., Awotunde, O.S., Swiatek, W., and Muszynska, G. (2001). Protein phosphatase 2A: variety of forms and diversity of functions. Acta Biochim. Pol. 48, 921-933. 
Lee, J., and Stock, J. (1993). Protein phosphatase 2A catalytic subunit is methyl-esterified at its carboxyl terminus by a novel methyltransferase. J. Biol. Chem. 268: 1919219195.

Levesque, D.L. and Tattersall, G.J. (2010). Seasonal torpor and normothermic energy metabolism in the Eastern chipmunk (Tamias striatus). J. Comp. Physiol. B 180: 279-292.

Li, S., Brown, M.S., and Goldstein, J.L. (2010). Bifurcation of insulin signalling pathway in rat liver: mTORC1 required for stimulation of lipogenesis, but not inhibition of gluconeogenesis. Proc. Natl. Acad. Sci. USA 107: 3441-3446.

Li, T., Liu, M., Feng, X., Wang, Z., Das, I., Xu, Y., Zhou, X., Sun, Y., Guan, K., Xiong, Y., and Lei, Q. (2013). Glyceraldehyde-3-phosphate dehydrogenase is activated by lysine 254 acetylation in response to glucose signal. J. Biol. Chem. 289: 37753785

Ljungstrom, O., Hjelmquist, G., and Engstrom, L. (1974). Phosphorylation of purified rat liver pyruvate kinase by cyclic-3',5'-AMP-stimulated protein kinase. Biochem. Biophys. Acta 358: 289-298.

Lv, L., Li, D., Zhao, D., Lin, R., Chu, Y., Zhang, H., Zha, Z., Liu, Y., Li, Z., Xu, Y., Wang, G., Huang, Y., Xiong, Y., Guan, K.L., Lei, Q.Y. (2011). Acetylation targets the M2 isoform of pyruvate kinase for degradation through chaperonemediated autophagy and promotes tumor growth. Mol. Cell. 42(6): 719-730.

Lyman, C.P., and Leduc, E.H. (1953). Changes in blood sugar and tissue glycogen in the hamster during arousal from hibernation. J. Cell. Comp. Physiol. 41: 471-492.

MacAulay, K., Doble, B.W., Patel, S., Hansotia, T., Sinclair, E.M., Drucker, D.J., Nagy, A., and Woodgett, J.R. (2007). Glycogen synthase kinase 3alpha-specific regulation of murine hepatic glycogen metabolism. Cell Metab. 6: 329-337.

MacDermott, M. (1990). The intracellular concentration of free magnesium in extensor digitorum longus muscles of the rat. Exp. Physiol. 75: 763-769.

MacDonald, J.A. (2004). Signal transduction pathways and the control of cellular responses to external stimuli, In: Functional Metabolism: Regulation and Adaptation (Ed. Kenneth B. Storey). Wiley-Liss, Inc., Hoboken, New Jersey. Pp. $87-120$.

MacDonald, M.J., and Chang, C. (1985). Pancreatic islets contain the M2 isozyme of pyruvate kinase - Its phosphorylation has no effect on activity. Mol. Cell. Biochem. 68: 115-120.

MacDonald, M.J. and Kowluru, A. (1985). Evidence for calcium enhanced phosphorylation of pyruvate kinase by pancreatic islets. Mol. Cell. Biochem. 68(2): 107-114.

MacDonald, J.A. and Storey, K.B. (1999). Regulation of ground squirrel $\mathrm{Na}^{+} / \mathrm{K}^{+}$ATPase activity by reversible phosphorylation during hibernation. Biochem. Biophys. Res. Comm. 254: 424-429. 
Mamady, H. and Storey, K.B. (2006). Up-regulation of the endoplasmic reticulum molecular chaperone GRP78 during hibernation in thirteen-lined ground squirrels. Mol. Cell. Biochem. 292: 89-98.

Mayer-Jaekel, R.E. and Hemmings, B.A. (1994). Protein phosphatase 2A - a "ménage à trois". Trends Cell Biol. 4: 287-291.

Mayya, V., Lundgren, D.H., Hwang, S.I., Rezaul, K., Wu, L., Eng, J.K., Rodionov, V., and Han, D.K. (2009). Quantitative phosphoproteomic analysis of T cell receptor signaling reveals system-wide modulation of protein-protein interactions. Sci. Signal. 2(84): ra46.

McArthur, M.D. and Milsom, W.K. (1991). Changes in ventilation and respiration sensitivity associated with hibernation associated with hibernation in Columbian (Spermophilus columbianus) and golden-mantled (Spermophilus lateralis) ground squirrels. Physiol. Zool. 64: 940-959.

McKeever, S. (1964). The biology of the golden-mantled ground squirrel, Citellus lateralis. Ecological Monographs 34: 383-401.

Minatogawa, Y., and Hue, L. (1984). Fructose-2,6-bisphosphate in rat skeletal muscle during contraction. Biochem. J. 223: 73-79.

Monera, O.D., Kay, C.M., and Hodges, R.S. (1994). Protein denaturation with guanidine hydrochloride or urea provides a different estimate of stability depending on the contributions of electrostatic interactions. Prot. Sci. 3: 1984-1991.

Mora, A., Sabio, G., Risco, A.M., Cuenda, A., Alonso, J.C., Soler, G., and Centeno, F. (2002). Lithium blocks PKB and GSK3 dephosphorylation induced by ceramide through protein phosphatase-2A. Cell Signal 14: 557-562.

Moreau-Hamsany, C., Castex, C., Hoo-Paris, R., Kacemi, N., and Sutter, B. (1988). Hormonal control of lipolysis from the white adipose tissue of hibernating jerboa (Jaculus orientalis). Comp. Biochem. Physiol. A Comp. Physiol. 91: 665-669.

Morrison, P.R. (1960). Some interrelations between weight and hibernation function. Bull. Museum Comp. Zool. Harvard College 124: 50-75.

Mostafa, N., Everett, D.C., Chou, S.C., Kong, P.A., Florant, G.L., and Coleman, R.A. (1993). Seasonal changes in critical enzymes of lipogenesis and triacylglycerol synthesis in the marmot (Marmota flaviventris). 163: 463-469.

Moy, R. (1971). Renal function in the hibernating ground squirrel Spermophilus columbianus. Am. J. Physiol. 220: 747-753.

Mukherjee, S., Keitany, G., Li, Y., Wang, Y., Ball, H.L., Goldsmith, E.J., and Orth, K. (2006). Yersinia YopJ acetylates and inhibits kinase activation by blocking phosphorylation. Science 312: 1211-1214.

Muise, A., and Storey, K.B. (1997). Reversible phosphorylation of fructose-1,6bisphosphatase mediates enzyme role in glycerol metabolism in the freezeavoiding gall moth Epiblema scudderiana. Insect Biochem. Mol. Biol. 27: 617- 
623.

Myles, T., Schmidt, K., Evans, D.R., Cron, P., and Hemmings, B.A. (2001). Active-site mutations impairing the catalytic function of the catalytic subunit of human protein phosphatase $2 \mathrm{~A}$ permit baculovirus-mediated overexpression in insect cells. Biochem. J. 357: 225-232.

Nakashima, K., Fujii, S., Kaku, K., and Kaneko, T. (1982). Calcium-calmodulin dependent phosphorylation of erythrocyte pyruvate kinase. Biochem. Biophys. Res. Comm. 104(1): 285-289.

Niesen, F.H., Berglund, H., and Vedadi, M. (2007). The use of differential scanning fluorimetry to detect ligand interactions that promote protein stability. Nature Protocols 2: 2212-2221.

Nishikawa, A., Kanehira, C., and Imamura, K. (2001). Purification and characterization of pyruvate kinase from lamprey (Entosphenus japonicas) muscle. Comp. Biochem. Physiol. B Biochem. Mol. Biol. 128(1): 159-164.

Noguchi, T., Inoue, H., and Tanaka, T. (1986). The M1- and M2-type isozymes of rat pyruvate kinase are produced from the same gene by alternative RNA splicing. $J$. Biol. Chem. 261: 13807-13812.

Obenauer, J.C., Cantley, L.C., and Yaffe, M.B. (2003). Scansite 2.0: Proteome-wide prediction of cell signaling interactions using short sequence motifs. Nucleic Acids Res. 31: 3635-3641.

Ormseth, O.A., Nicolson, M., Pelleymounter, M., and Boyer, B.B. (1996). Leptin inhibits prehibernation hyperphagia and reduces body weight in artic ground squirrels. Am. J. Physiol. 271: R1775-R1779.

Palumbo, P.J., Wellik, D.L., Bagley, N.A., and Nelson, R.A. (1983). Insulin and glucagon responses in the hibernating black bear. Int. Conf. Bear Res. Manag. 291-296.

Patel, S., Doble, B.W., MacAuley, K., Sinclair, E.M., Drucker, D.J., and Woodgett, J.R. (2008). Tissue-specific role of glycogen synthase kinase 3 beta in glucose homeostasis and in insulin action. Mol. Cell Biol. 28: 6314-6328.

Picton, C., Woodgett, J., Hemmings, B., and Cohen, P. (1982). Multisite phosphorylation of glycogen synthase from rabbit skeletal muscle. Phosphorylation of site 5 by glycogen synthase kinase-5 (casein kinase-II) is a prerequisite for phosphorylation of sites 3 by glycogen synthase kinase-3. FEBS Lett. 150: 191-196.

Pilkis, S.J., El-Maghrabi, M.R., McGrane, M.M., Pilkis, J., Claus, T.H. (1981). The role of fructose-2,6-bisphosphate in regulation of fructose-1,6-bisphosphatase. J. Biol. Chem. 256, 3619-3622.

Plaxton, W.C. and Storey, K.B. (1984). Phosphorylation in vivo of red-muscle pyruvate kinase from the channelled whelk, Busycotpus canaliculatum, in response to anoxic stress. Eur. J. Biochem. 143: 267-272. 
Porstmann, T., Santos, C.R., Griffiths, B., Cully, M., Wu, M., Leevers, S., Griffiths, J.R., Chung, Y.L., and Schultze, A. (2008). SREBP activity is regulated by mTORC1 and contributes to Akt-dependent cell growth. Cell Metab. 8: 224-236.

Pontremoli, S. and Horecker, B.L. (1971). Fructose-1,6-diphosphatase from rabbit liver. Curr. Top. Cell Regul. 2: 173-199.

Prendergast, B.J., Freeman, D.A., Zucker, I., and Nelson, R.J. (2002). Periodic arousal from hibernation is necessary for initiation of immune responses in ground squirrels. Am. J. Physiol. Regul. Integr. Comp. Physiol. 282: R1054-R1062.

Presek, P., Reinacher, M., and Eigenbrodt, E. (1988). Pyruvate kinase type M2 is phosphorylated at tyrosine residues in cells transformed by Rous sarcoma virus. FEBS Lett. 242(1): 194-198.

Primot, A., Baratte, B., Gompel, M., Borgne, A., Liabeuf, S., Romette, J.L., Jho, E.H., Constantini, F., and Meijer, L. (2000). Purification of GSK-3 by affinity chromatography on immobilized axin. Protein Expr. Purif. 20: 394-404.

Qian, W., Shi, J., Yin, X., Iqbal, K., Grundke-Iqbal, I., Gong, C.X., and Liu, F. (2010). PP2A regulates tau phosphorylation directly and also indirectly via activating GSK-3beta. J. Alzheimers Dis. 19: 1221-1229.

Rakus, D., and Dzugaj, A. (2000). Muscle aldolase decreases muscle FBPase sensitivity toward AMP inhibition. Biochem. Biophys. Res. Commun. 275: 611-616.

Rakus, D., Zarzycki, M., and Dzugai, A. (2003). Rabbit muscle fructose-1,6bisphosphatase is phosphorylation in vivo. Acta Biochim Pol. 50: 115-121.

Ramnanan, C.J. Groom, A.G., and Storey, K.B. (2007). Akt and its downstream targets play key roles in mediating dormancy in land snails. Comp. Biochem. Physiol. B. 148: $245-255$.

Randall, R.F. and Anderson, P.J. (1975). Purification and properties of the pyruvate kinase of sturgeon muscle. Biochem. J. 145(3): 569-573.

Raymond, K.W. (2010). General Organic and Biological Chemistry: An Integrated Approach $3^{\text {rd }}$ Edition. John Wiley and Sons Inc.

Riou, J.P., Claus, T.H., and Pilkis, S.J. (1978). Stimulation of glucagon of an in vivo phosphorylation of rat hepatic pyruvate kinase. J. Biol. Chem. 253: 656-659.

Rocco, A.G., Mollica, L., Ricchiuto, P., Baptista, A.M., Gianazza, E., and Eberini, I. (2008). Characterization of the protein unfolding processes induced by urea and temperature. Biophys. J. 94: 2241-2251.

Rose, M., Entian, K., Hofmann, L., Vogel, R.F., and Mecke, D. (1988). Irreversible inactivation of Saccharomyces cerevisae fructose-1,6-bisphosphatase independent of protein phosphorylation at Ser11. independent of protein phosphorylation at Ser11. FEB 241: 55-59.

Rylatt, D.B. Aitken, A., Bilham, T., Condon, G.D., Embi, N., and Cohen, P. (1980). Glycogen synthase from rabbit skeletal muscle. Amino acid sequence at the sites phosphorylated by glycogen synthase kinase-3, and extension of the N-terminal 
sequence containing the site phosphorylated by phosphorylase kinase. Euro. $J$. Biochem. 107: 529-537.

Sadoul, K., Wang, J., Diagouraga, B., and Khochbin, S. (2010). The tale of protein lysine acetylation in the cytoplasm. J. Biomed. Biotech. Article ID 970382.

Sarbassov, D.D., Guertin, D.A., Ali, S.M., and Sabatini, D.M. (2005). Phosphorylation and regulation of Akt/PKB by the rictor-mTOR complex. Science 307: 10981101.

Seo, J., Jeong, J., Kim, Y.M., Hwang, N., Paek, E., and Lee, K. (2007). Strategy of comprehensive identification of post-translational modifications in cellular proteins, including the low abundant modifications: Application to glyceraldehyde-3-phosphate dehydrogenase. J. Proteome Res. 7: 587-602.

Sergienko, E.A., Kharitonekov, A.I., Bulgarina, T.V., Muronetz, V.I. and Nagradova, N.K. (1992). D-glyceraldehyde-3-phosphate dehydrogenase purified from rabbit muscle contains phosphotyrosine. FEBS Lett. 231: 413-416.

Simon, G.M., Cheng, J., and Gordon, J.I. (2012). Quantitative assessment of the impact of gut microbiota on lysine epsilon-acetylation of host gnotobiotic mice. Proc. Natl. Acad. Sci. USA 109: 11133-11138.

Singh, P., Salih, M., Leddy, J.J., and Tuana, B.S. (2004). The muscle-specific calmodulin-dependent protein kinase assembles with the glycolytic enzyme complex at the sarcoplasmic reticulum and modulates the activity of glyceraldehyde-3-phosphate dehydrogenase in a $\mathrm{Ca} 2+/$ calmodulin-dependent manner. J. Biol. Chem. 279(34): 35176-35182.

Sirover, M.A. (2011). On the functional diversity of glyceraldehyde-3-phosphate dehydrogenase: Biochemical mechanisms and regulatory control. Biochim Biophys. Acta 1810: 741-751.

Skurat, A.V., and Roach, P.J. (1995). Phosphorylation of sites 3a and 3b (Ser(640) and Ser(644)) in the control of rabbit muscle glycogen synthase. J. Biol. Chem. 270: 12491-12497.

Skurat, A.V. and Dietrich, A.D. (2004). Phosphorylation of Ser640 in muscle glycogen synthase by DYRK family protein kinases. J. Biol. Chem. 279: 2490-2498.

Smith, C.R., Knowles, V.L., and Plaxton, W.C. (2000). Purification and characterization of cytosolic pyruvate kinase from Brassica napus (rapeseed) suspension cell cultures. Eur. J. Biochem. 267: 4477-4486.

Srere. H.K., Wang, L.C.H., and Martin, S.L. (1992). Central role for differential gene expression in mammalian hibernation. Proc. Natl. Acad. Sci USA 89: 7119-7123.

Stadtman, E.R., Moskovitz, J., and Levine, R.L. (2003). Oxidation of methionine residues of proteins: Biological consequences. Antioxidants and Redox Signaling 5: 577582. 
Stambolic, V., Ruel, L., and Woodgett, J.R. (1996). Lithium inhibits glycogen synthase kinase-3 activity and mimics Wingless signaling in intact cells. Curr. Biol. 6: 1664-1669.

Staples, J.F. and Hochachka, P.W. (1998). The effect of hibernation status and coldacclimation on hepatocyte gluconeogenesis in the golden-mantled ground squirrel (Spermophilus lateralis). Can. J. Zool. 76: 1734-1740.

Storey, K.B. (1987). Regulation of liver metabolism by enzyme phosphorylation during mammalian hibernation. J. Biol. Chem. 262: 1670-1673.

Storey, K.B. (1989). Integrated control of metabolic rate depression via reversible phosphorylation of enzymes in hibernating mammals. In: Living in the Cold II (eds. Malan, A. and Canguilhem, B.). pp. 309-319. John Libbey Eurotext, London.

Storey, K.B. (2010). Out cold: Biochemical regulation of mammalian hibernation - A mini-review. Gerontology 56: 220-230.

Storey, K.B. and Kelly, D.A. (1995). Glycolysis and energetic in organs of hibernating mice (Zapus hudsonius). Can. J. Zool. 73: 202-207.

Storey, K.B. and Storey, J.M. (2010). Metabolic rate depression: the biochemistry of mammalian hibernation. Advan. Clin. Chem. 52: 77-108.

Stralfors, P., and Honner, R.C. (1989). Insulin-induced dephosphorylation of hormonesensitive lipase. Correlation with lipolysis and cAMP-dependent protein kinase activity. Eur. J. Biochem. 182: 379-385.

Su, B., Wang, X., Drew, K.L., Perry, G., Smith, M.A., and Zhu, X. (2008). Physiological regulation of tau phosphorylation during hibernation. J. Neurochem. 105: 20982108.

Sutherland, C., Leighton, I.A., and Cohen, P. (1993). Inactivation of glycogen synthase kinase $3 \beta$ by phosphorylation: New kinase connections in insulin and growthfactor signaling. Biochem 296: 15-19.

Tashima, L.S. Adelstein, S.J., and Lyman, C.P. (1970). Radioglucose utilization by active, hibernating, and arousing ground squirrels. Am. J. Physiol.218: 303-309.

Thomas, S. and Fell, D.A. (1998). The role of multiple enzyme activation in metabolic flux control. Adv. Enzyme Regul. 38: 65-85.

Thornton, T.M., Pedraza-Alva, G., Deng, B., Wood, C.D., Aronshtam, A., Clements, J.L., Sabio, G., Davis, R.J., Matthews, D.E., Doble, B., and Rincon, M. (2008). Phosphorylation by 38 MAPK as an alternative pathway for GSK3beta inactivation. Science 320: 667-670.

Tisdale, E.J. (2002). Glyceraldehyde-3-phosphate dehydrogenase is phosphorylated by protein kinase $\mathrm{Cl} / \lambda$ and plays a role in microtubule dynamics in the early secretory pathway. J. Biol. Chem.277: 3334-3341. 
Tisdale, E.J., and Artalejo, C.R. (2007). GAPDH mutant defective in Src-dependent tyrosine phosphorylation impedes Rab2-mediated events. Traffic 8: 733-741.

Titanji, V.P.K., Zetterqvist, O., and Engstrom, L. (1976). Regulation in vitro of rat liver pyruvate kinase by phosphorylation-dephosphorylation reactions, catalyzed by cyclic-AMP dependent protein kinases and a histone phosphatase. Biochim. Biophys. Acta 422(1): 98-108.

Tristan, C., Shahani, N., Sedlak, T.W., and Sawa, A. (2011). The diverse functions of GAPDH: Views from different subcellular compartments. Cellular Signaling 23: 317-323.

Underwood, H., and Newsholme, E.A. (1965). Some properties of fructose-1,6diphosphate of rat liver and their relation to the control of gluconeogenesis. Biochem. J. 96: 767-774.

Vaillancourt, E., Haman, F., and Weber, J. (2009). Fuel selection in Wistar rats exposed to cold: shivering thermogenesis diverts fatty acids from re-esterification to oxidation. J. Physiol. 587: 4349-4359.

van Breukelen, F., Sonenberg, N., and Martin, S.L. (2004). Seasonal and state-dependent changes of eI4E and 4E-BP1 during mammalian hibernation: implications for the control of translation during torpor. Am. J. Physiol. Regul. Integr. Comp. Physiol. 287: 349-353.

van Noort, V., Seebacher, J., Bader, S., Mohammed, S., Vonkova, I., Betts, M.J., Kuhner, S., Kumar, R., Maier, T., O’Flaherty, M., Rybin, V., Schmeisky, A., Yus, E., Stulke, J., Serrano, L., Russell, R.B., Heck, A.J.R., Bork, P., and Gavin, A. (2012). Cross-talk between phosphorylation and lysine acetylation in a genomereduced bacterium. Mol. Syst. Biol. 8: 571. DOI: 10.1038/msb.2012.4

van Schaftingen, E. and Hers, H.G. (1981). Inhibition of fructose-1,6-bisphosphatase by fructose-2,6-bisphosphate. Proc. Natl. Acad. Sci. USA 78: 2861-2863.

Ventura, M., Mateo, F., Serratosa, J., Salaet, J., Salaet, I., Carujo, S., Bachs, O., and Pujol, M.J. (2010). Nuclear translocation of glyceraldehydes-3-phosphate dehydrogenase is regulated by acetylation. Int. J. Biochem. Cell Biol. 42: 16721680 .

Virshup, D.M. (2000). Protein phosphatase 2A: a panoply of enzymes. Curr. Opin. Cell Biol. 12, 180-185.

Walter, L. and Bienz, M. (1998). Drosophila CBP represses the transcription factor TCF to antagonize Wingless signalling. Nature 395(6701): 521-525.

Wang, L.C.H. (1979). Time patterns and metabolic rates of natural torpor in Richardson's ground squirrel. Can. J. Zool. 57: 149-155.

Wang, P., Walter, R.D., Bhat, B.G., Florant, G.L., and Coleman, R.A. (1998). Seasonal changes in enzymes of lipogenesis and triacylglycerol synthesis in the golden- 
mantled ground squirrel (Spermophilus lateralis). Comp. Biochem. Physiol. B Biochem. Mol. Biol. 118: 261-267.

Wang, Q., Zhang, Y., Yang, C., Xiong, H., Lin, Y., Yao, J., Li, H., Xie, L., Zhao, W., Yao, Y., Ning, Z., Zeng, R., Xiong, Y., Guan, K., Zhao, S., and Zhao, G. (2010). Acetylation of metabolic enzymes coordinates carbon source utilization and metabolic flux. Science 327: 1004-1008.

Weernink, P.A., Rijksen, G., van der Heijden, M.C. and Staal, G.E. (1990). Phosphorylation of pyruvate kinase type $\mathrm{K}$ in human gliomas by a cyclic adenosine 5'-monophosphate-independent protein kinase. Cancer Res. 50(15): 4604-4610.

White, A.T., and Schenik, S. (2012). NAD+/NADH and skeletal muscle mitochondrial adaptations to exercise. Am. J. Physiol. Endocrinol. Metab. 303: E308-E321.

Willis, J.S. Goldman, S.S., and Foster, R.F. (1971). Tissue K concentration in relation to the role of the kidney in hibernation and the cause of periodic arousal. Comp. Biochem. Physiol. A: Physiol. 39(3): 437-445.

Woods, A.K. and Storey, K.B. (2004). Effects of hibernation on the multicatalytic proteinase complex in thirteen-lined ground squirrels, Spermophilus tridecemlineatus. Mol. Cell. Biochem. 271: 205-213.

Wu, C. and Storey, K.B. (2012a). Pattern of cellular quiescence over the hibernation cycle in liver of thirteen-lined ground squirrels. Cell Cycle 11: 1714-1726.

Wu, C. and Storey, K.B. (2012b). Regulation of the mTOR signaling network in hibernating thirteen-lined ground squirrel. J. Exp. Biol. 215: 1720-1727.

$\mathrm{Xie}, \mathrm{H}$. and Clarke, S. (1994). Protein phosphatase 2A is reversibly modified by methyl esterification at its C-terminal leucine residue in bovine brain. J. Biol. Chem. 269: 1981-1984.

Xiong, Y., Lei, Q.Y., Zhao, S., and Guan, K.L. (2011). Regulation of glycolysis and gluconeogenesis by acetylation of PKM and PEPCK. Cold Spring Harb. Symp. Quant. Biol. 76: 285-289.

Yan, L., Lavin, V.A., Moser, L.R., Cui, Q., Kaines, C., and Yang, E. (2008). PP2A regulates the pro-apoptotic activity of FOXO1. J. Biol. Chem. 283, 7411-7420.

Yang, X., and Seto, E. (2008). Lysine acetylation: codified crosstalk with other posttranslational modifications. Mol. Cell. 31: 449-461.

Zatman, M.L. (1984). Renal and cardiovascular effects of hibernation and hypothermia. Cryobiology 21: 593-614.

Zhang, X., Wen, H., and Shi, X. (2012). Lysine methylation: beyond histones. Arch. Biochem. Biophys. Sin. 44, 14-27.

Zhao, S., Xu, W., Jiang, W., Yi, W., Lin, Y., Zhang, T., Yao, J., Zhou, L., Zeng, Y., Li, H., Li, Y., Shi, J., An, W., Hancock, S.M., He, F., Qin, L., Yang, P., Chen, X., Lei, Q., Xiong, Y., and Guan, K.L. (2010). Regulation of cellular metabolism by protein lysine acetylation. Science 327: 1000-1004. 
Zhou, H., Palma, S., Preisinger, C., Peng, M., Polat, A.N., Heck, A.J., Mohammed, S. (2013). Toward a comprehensive characterization of a human cancer cell phosphoproteome. J. Proteome Res. 12: 260-271.

Zimmerman, M.L. (1982). Carbohydrate and torpor duration in hibernating goldenmantled ground squirrels (Citellus lateralis). J. Comp. Physiol. B 147: 129-135.

Zimny, M.L. and Tyrone, V. (1957). Carbohydrate metabolism during fasting and hibernation in the ground squirrel. Am. J. Physiol. 189: 297-300.

Zivadinovic, D., Marjanovic, M. and Andjus, R.K. (2005). Some components of hibernation rhythms. Ann. NY Acad Sci. 1048: 60-68. 


\section{Appendix I}

\section{Supplementary Information from Thesis Chapters}


Appendix IA: The deduced amino acid sequence for 13-lined ground squirrel GAPDH, with sequences that were identified for ACR GAPDH by mass spectrometry highlighted in yellow.

$1 \quad$ M V K V G V N G F G R I G R L V T R A A

21 F K S G K V D I V A I N D P F I D L N Y

41 M V Y M F Q Y D S T H G K F N G T V K A

61 E N G K L V I N G K S I S I F Q E R D P

81 A N I K W G D A G A E Y V V E S T G V F

101 T T M E K A G A H L K G G A K R V I I S

121 A S S A D A P M F V M T V N H E M Y D N

141 S P K I I S N A S C T T N C L I P L A K

161 V I H D N S S I V E G L K T T V L V I T

181 T N Q K T V D G P S G K L W H D G D G A

201 A Q N I L S A S T G A A K A V G K V I P

221 R L N G K L T G M A F R V P T P N V S V

241 V D L T C R L E K A A K Y D D I K K V V

261 K Q A S E G P L K G I L G Y T E D Q V V

281 S C D F N S D I H S S T F D A G A G I A

301 L N D H F V K L I S W Y D N E F G Y S N

321 R V V D L M V H M A S K E* 
Appendix IB: The deduced amino acid sequence for 13-lined ground squirrel GAPDH, with LH GAPDH sequences that were identified by mass spectrometry highlighted in yellow.

$1 \quad$ M V K V G V N G F G R I G R L V T R A A 21 F K S G K V D I V A I N D P F I D L N Y

41 M V Y M F Q Y D S T H G K F N G T V K A

61 E N G K L V I N G K S I S I F Q E R D P

81 A N I K W G D A G A E Y V V E S T G V F

101 T T M E K A G A H L K G G A K R V I I S

121 A S S A D A P M F V M T V N H E M Y D N

141 S P K I I S N A S C T T N C L I P L A K

161 V I H D N S S I V E G L K T T V L V I T

181 T N Q K T V D G P S G K L W H D G D G A

201 A Q N I L S A S T G A A K A V G K V I P

221 R L N G K L T G M A F R V P T P N V S V

241 V D L T C R L E K A A K Y D D I K K V V

261 K Q A S E G P L K G I L G Y T E D Q V V

281 S C D F N S D I H S S T F D A G A G I A

301 L N D H F V K L I S W Y D N E F G Y S N

321 R V V D L M V H M A S K E * 
Appendix IC: Thirteen-lined ground squirrel PK amino acid sequence obtained from the low-coverage sequencing attempt by the Broad Institute.

MSKPHSEAGTAFIQTQQLHAAMADTFLEHMCRLDIDSPPITARNTGIICTIGP ASRSVEMLKEMI KSGMNVARLNFS HGTHEYHAETIKNVRAATESFASDP I LYRPV AVALDTKGPEIRTGLIKGSGTAEVELKKGATLKITLDNSYMEKCDENI LWLDYKN ICKVVEVGSKIYVDDGLISLLVKEKGADFLVTEVENGGSLGSKKGVNLPGAAVDL PAVSEKDIQDLKFGVEQDVDMVFASFIRKASDVHEVRKI LGEKGKNIKI ISKIEN HEGVRRFDEILEASDGIMVARGDLGIEI PAEKVFLAQKMMI GRCNRAGKPVICAT QMLESMIKKPRPTRAEGSDVANAVLDGADCIMLSGETAKGDYPLEAVRMQHLIAR EAEAAMYHSKLFEELARASCLSTDPMEAMAMGSVEASYKCLAAALIVLTESGRSA HQVARYRPRAPI IAVTRNPQTARQAHLYRGIFPVVCKDPVQDAWAEDVDLRVNLA MNVGKARGFFKKGDVVIVLTGWRPGSGFTNTMRVVPVP 
APPENDIX ID: Pyruvate Kinase NetPhos 2.0 output for possible phosphorylation sites using the 13-lined ground squirrel sequence.

MSKPHSEAGTAF IQTQQLHAAMADTFLEHMCRLDI DSPPITARNTGI I TT IGPAS RSVEMLKEMIKS GMNVARLNFSHGT 80 HEYHAETIKNVRAATESFAS DP I LYRPVAVALDTKGPEIRTGLIKGSGTAEVELK KGATLKITLDNSYMEKCDENILWLD 160 YKN I CKVVEVGSKIYVDDGL I S LLVKEKGADF LVTEVENGGS LGSKKGVNLPGAA VDLPAVSEKDIQDLKFGVEQDVDMV 240

FASFIRKAS DVHEVRKI LGEKGKN IKI I SKIENHEGVRRF DEI LEASDG IMVARG DLGIEIPAEKVFLAQKMMIGRCNRA 320

GKPVICATQMLESMIKKPRPTRAEGSDVANAVLDGADC IMLSGETAKGDYPLEAV RMQHLIAREAEAAMYHSKLFEELAR 400

ASCLSTDPMEAMAMGSVEASYKCLAAAL IVLTESGRSAHQVARYRPRAP I IAVTR NPQTARQAHLYRGIFPVVCKDPVQD 480

AWAEDVDLRVNLAMNVGKARGFFKKGDVVIVLTGWRPGSGFTNTMRVVPVP

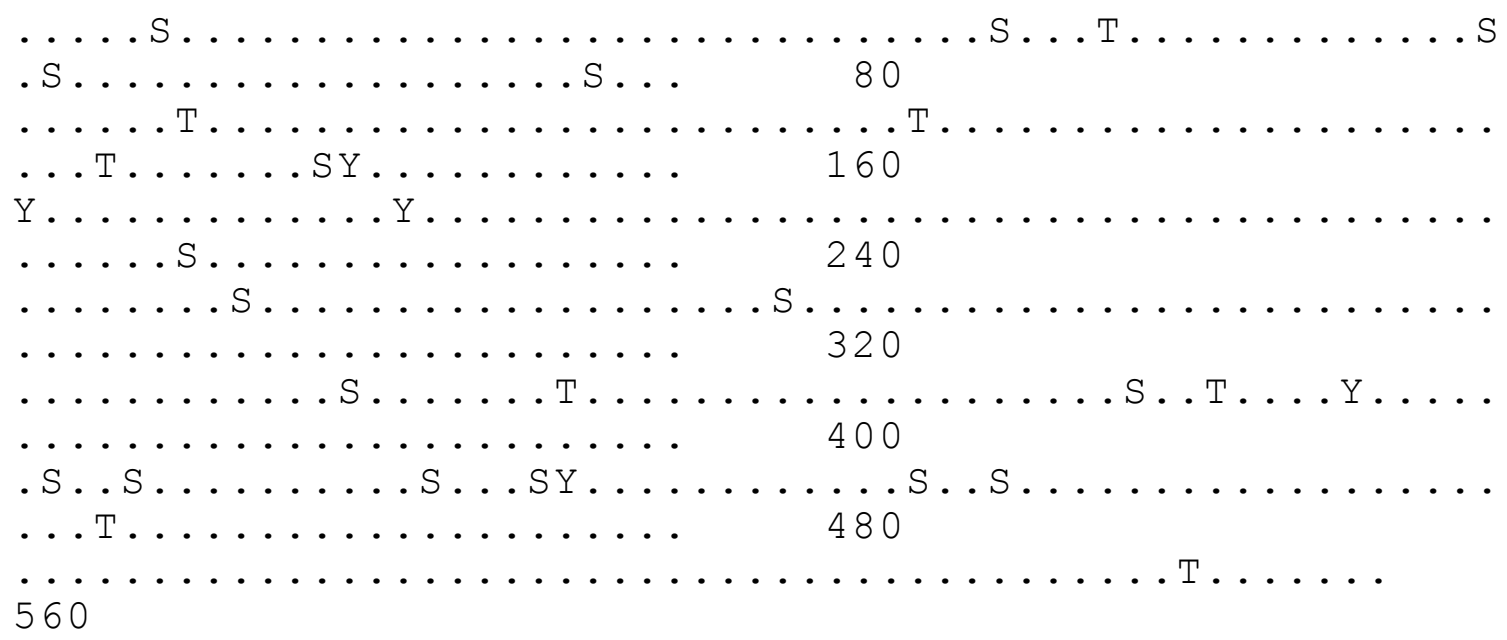

Phosphorylation sites predicted: Ser: 17 Thr: 8 Tyr: 5

Serine predictions

\begin{tabular}{|c|c|c|c|c|}
\hline Name & Pos & $\begin{array}{c}\text { Context } \\
\mathrm{V}\end{array}$ & score & Preo \\
\hline$\overline{\mathrm{P}} \overline{\mathrm{K}}$ & $\overline{2}$ & $---\bar{M} S \bar{K} \bar{P} \bar{H} \bar{S}$ & $\overline{0} . \overline{0} \overline{5}$ & • \\
\hline $\mathrm{PK}$ & 6 & SKPHSEAGT & 0.992 & $\star S *$ \\
\hline $\mathrm{PK}$ & 37 & LDIDSPPIT & 0.578 & $\star{ }^{*}$ * \\
\hline $\mathrm{PK}$ & 55 & IGPASRSVE & 0.845 & $\star S *$ \\
\hline $\mathrm{PK}$ & 57 & PASRSVEML & 0.982 & $\star S *$ \\
\hline $\mathrm{PK}$ & 67 & EMI KS GMNV & 0.036 & 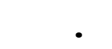 \\
\hline $\mathrm{PK}$ & 77 & RLNFSHGTH & 0.594 & $\star S *$ \\
\hline $\mathrm{PK}$ & 97 & AATESFASD & 0.113 & . \\
\hline $\mathrm{PK}$ & 100 & ESFASDPIL & 0.042 & . \\
\hline PK & 127 & LIKGSGTAE & 0.185 & . \\
\hline
\end{tabular}




\begin{tabular}{|c|c|c|c|c|}
\hline PK & 147 & TLDNSYMEK & 0.975 & $\star S *$ \\
\hline $\mathrm{PK}$ & 172 & VEVGSKIYV & 0.021 & . \\
\hline PK & 182 & DGL I S LLVK & 0.010 & . \\
\hline PK & 202 & ENGGSLGSK & 0.026 & . \\
\hline PK & 205 & GSLGSKKGV & 0.118 & . \\
\hline $\mathrm{PK}$ & 222 & LPAVSEKDI & 0.997 & $\star S *$ \\
\hline $\mathrm{PK}$ & 243 & MVFASFIRK & 0.036 & • \\
\hline PK & 249 & IRKASDVHE & 0.940 & $\star S *$ \\
\hline $\mathrm{PK}$ & 269 & IKI ISKIEN & 0.591 & $\star S *$ \\
\hline $\mathrm{PK}$ & 287 & I LEASDG IM & 0.016 & . \\
\hline $\mathrm{PK}$ & 333 & QMLESMIKK & 0.645 & $\star S *$ \\
\hline $\mathrm{PK}$ & 346 & RAEGSDVAN & 0.407 & . \\
\hline $\mathrm{PK}$ & 362 & CIMLSGETA & 0.584 & $\star S *$ \\
\hline $\mathrm{PK}$ & 392 & AMYHSKLFE & 0.076 & . \\
\hline $\mathrm{PK}$ & 402 & LARASCLST & 0.910 & $\star S *$ \\
\hline PK & 405 & ASCLSTDPM & 0.542 & $\star S *$ \\
\hline $\mathrm{PK}$ & 416 & MAMGSVEAS & 0.637 & $\star S *$ \\
\hline PK & 420 & SVEASYKCL & 0.983 & $\star S *$ \\
\hline PK & 434 & VLTESGRSA & 0.918 & $\star S *$ \\
\hline $\mathrm{PK}$ & 437 & ESGRSAHQV & 0.720 & $\star S *$ \\
\hline PK & 519 & WRPGSGFTN & 0.343 & • \\
\hline
\end{tabular}

Threonine predictions

\begin{tabular}{|c|c|c|c|c|}
\hline Name & Pos & $\begin{array}{c}\text { Context } \\
V\end{array}$ & Score & Preo \\
\hline$\overline{\mathrm{P}} \overline{\mathrm{K}}$ & $\overline{1} 0$ & $\overline{\mathrm{S}} \overline{\mathrm{E}} \overline{\mathrm{A}} \overline{\mathrm{G}} \mathrm{T} \overline{\mathrm{A}} \overline{\mathrm{F}} \overline{\mathrm{I}} \overline{\mathrm{Q}}$ & $0 . \overline{0} \overline{4}$ & $\bullet$ \\
\hline $\mathrm{PK}$ & 15 & AF I QTQQLH & 0.055 & • \\
\hline $\mathrm{PK}$ & 25 & AMADTFLEH & 0.231 & . \\
\hline $\mathrm{PK}$ & 41 & SPPITARNT & 0.878 & $\star \mathrm{T} *$ \\
\hline $\mathrm{PK}$ & 45 & TARNTGI I C & 0.115 & . \\
\hline $\mathrm{PK}$ & 50 & GIICTIGPA & 0.193 & . \\
\hline $\mathrm{PK}$ & 80 & FSHGTHEYH & 0.029 & . \\
\hline $\mathrm{PK}$ & 87 & YHAETIKNV & 0.924 & $\star \mathrm{T} *$ \\
\hline $\mathrm{PK}$ & 95 & VRAATESFA & 0.480 & . \\
\hline $\mathrm{PK}$ & 114 & VALDTKGPE & 0.782 & $\star T *$ \\
\hline $\mathrm{PK}$ & 121 & PEIRTGLIK & 0.031 & . \\
\hline $\mathrm{PK}$ & 129 & KGSGTAEVE & 0.292 & . \\
\hline $\mathrm{PK}$ & 139 & KKGATLKIT & 0.634 & $\star T^{*}$ \\
\hline PK & 143 & TLKITLDNS & 0.019 & . \\
\hline $\mathrm{PK}$ & 195 & DFLVTEVEN & 0.498 & . \\
\hline $\mathrm{PK}$ & 328 & VICATQMLE & 0.046 & . \\
\hline $\mathrm{PK}$ & 341 & KPRPTRAEG & 0.843 & $\star T^{*}$ \\
\hline PK & 365 & LSGETAKGD & 0.611 & $\star T *$ \\
\hline $\mathrm{PK}$ & 406 & SCLSTDPME & 0.016 & . \\
\hline $\mathrm{PK}$ & 432 & LIVLTESGR & 0.084 & . \\
\hline $\mathrm{PK}$ & 454 & I I AVTRNPQ & 0.027 & . \\
\hline $\mathrm{PK}$ & 459 & RNPQTARQA & 0.931 & $\star T *$ \\
\hline
\end{tabular}




$\begin{array}{llccc}\text { PK } & 513 & \text { VIVLTGWRP } & 0.099 & . \\ \text { PK } & 522 & \text { GSGFTNTMR } & 0.015 & * \\ \text { PK } & 524 & \text { GFTNTMRVV } & 0.945 & * \text { * * }\end{array}$

Tyrosine predictions

\begin{tabular}{|c|c|c|c|c|}
\hline Name & Pos & $\begin{array}{c}\text { Context } \\
\mathrm{V}\end{array}$ & Score & Pred \\
\hline$\overline{\mathrm{P}} \overline{\mathrm{K}}$ & $8 \overline{3}$ & $\overline{\mathrm{G}} \overline{\mathrm{T}} \overline{\mathrm{H}} \overline{\mathrm{E}} \mathrm{Y} \overline{\mathrm{H}} \overline{\mathrm{A}} \overline{\mathrm{E}} \overline{\mathrm{T}}$ & $0.0 \overline{2}$ & 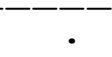 \\
\hline PK & 105 & DPI LYRPVA & 0.075 & • \\
\hline PK & 148 & LDNSYMEKC & 0.552 & $\star Y *$ \\
\hline PK & 161 & LWLDYKN I C & 0.632 & $\star Y *$ \\
\hline $\mathrm{PK}$ & 175 & GSKIYVDDG & 0.873 & $\star Y *$ \\
\hline $\mathrm{PK}$ & 370 & AKGDYPLEA & 0.559 & $\star Y *$ \\
\hline PK & 390 & EAAMYHSKL & 0.273 & • \\
\hline PK & 421 & VEASYKCLA & 0.867 & $\star Y *$ \\
\hline $\mathrm{PK}$ & 444 & QVARYRPRA & 0.009 & . \\
\hline $\mathrm{PK}$ & 466 & $\underset{\wedge}{Q A H L Y R G I F}$ & 0.011 & . \\
\hline
\end{tabular}

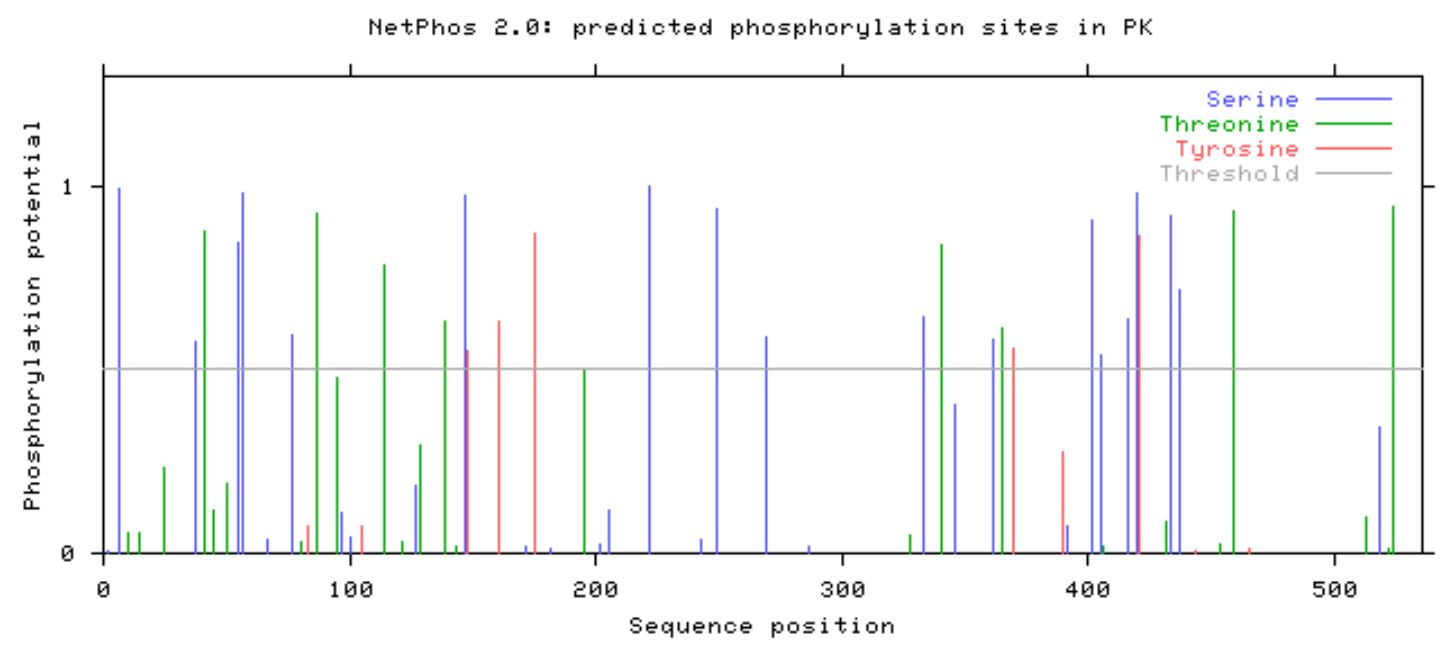


Appendix IE: Ramachandran plot for 13-lined ground squirrel PP2Ac modeled after human PP2Ac (PDB: 2IE3.c).

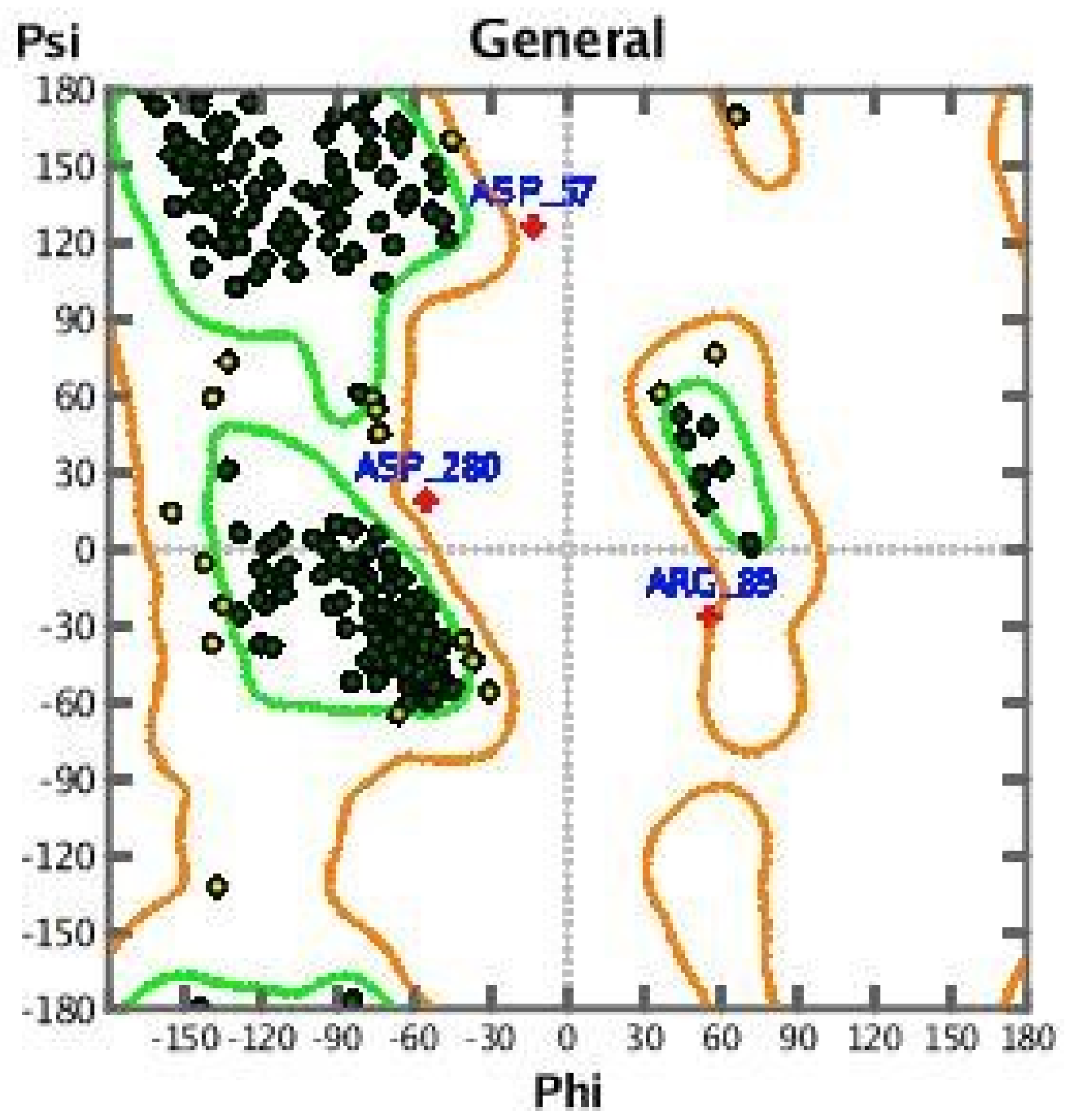


Appendix IF: The glycolytic and gluconeogenic pathways. The glycolytic pathway proceeds from top to bottom and the gluconeogenic pathway proceeds from bottom to top. The diagram was taken from Raymond (2009).

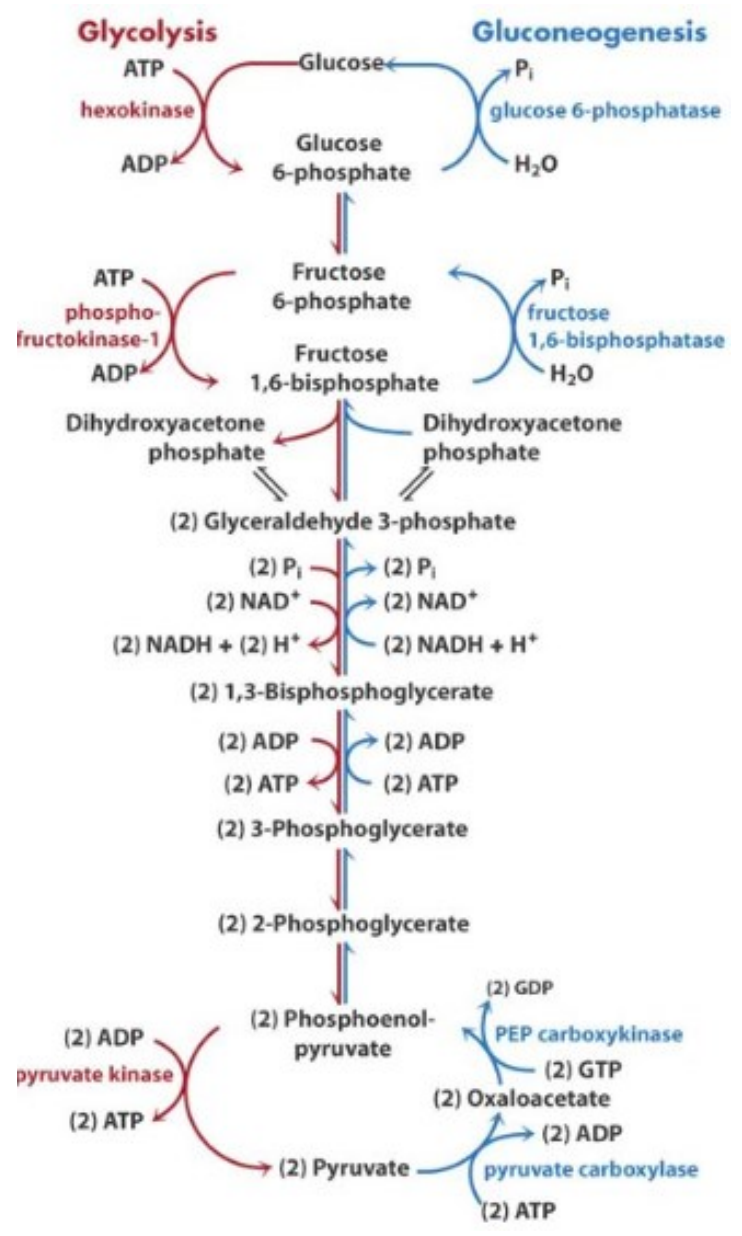


Appendix IG: The specific reactions catalyzed by the enzymes studied in this thesis (Chapters 2-4). The GAPDH and PK reaction illustrations were taken from www.chem.uwec.edu. The FBPase reaction illustration was taken from www2.ufp.pt.

Glyceraldehyde-3-phosphate Dehydrogenase

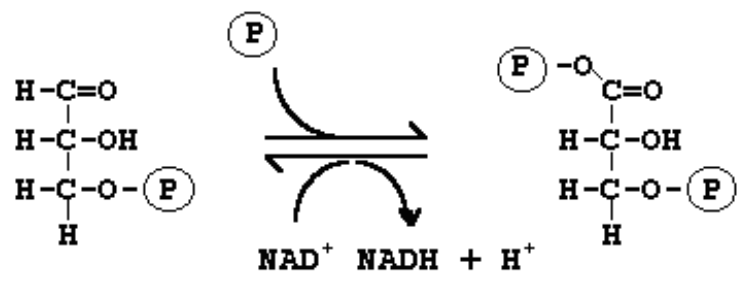

Glyceraldehyde 3-phosphate

1,3-Bisphosphoglycerate

\section{Pyruvate Kinase}

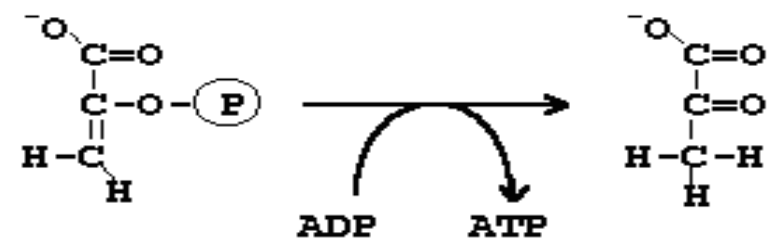

Phosphoenolpyruvate

Pyruvate

Fructose-1,6-bisphosphatase

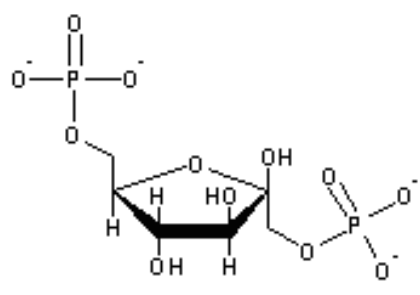

Frutose-1,6-BP

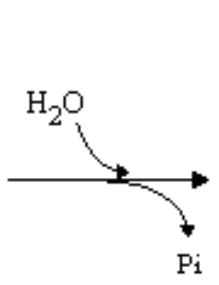

$\mathrm{Pi}$

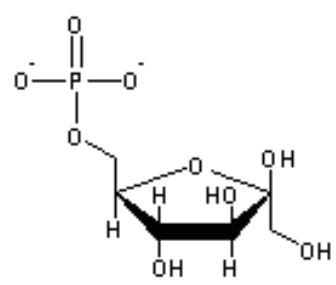

Frutose-6-P 
Appendix II List of Publications 


\section{Publications (published):}

[1] Shahriari, A., Dawson, N.J., Bell, R.A.V., and Storey, K.B. Stable suppression of lactate dehydrogenase activity in the foot muscle of Littorina littorea and the potential role of acetylation as a novel posttranslational regulatory mechanism. Enz. Res. doi: $10.1155 / 2013$

[2] Lama, J.L., Bell, R.A.V., and Storey, K.B. (2013). Hexokinase regulation in the hepatopancreas and foot muscle of the anoxia-tolerant marine mollusc, Littorina littorea. Comp. Biochem. Physiol. B Biochem Mol. Biol. Accepted July 4 ${ }^{\text {th }}, 2013$.

[3] Bell, R.A.V., Dawson, N.J., and Storey, K.B. (2013). Purification and properties of white muscle lactate dehydrogenase from the anoxia-tolerant turtle, the red-eared slider, Trachemys scripta elegans. Enz. Res. Article ID 784973.

[4] Lama, J.L., Bell, R.A.V., and Storey, K.B. (2013). Glucose-6-phosphate dehydrogenase regulation in the hepatopancreas of the anoxia-tolerant marine mollusc, Littorina littorea. PeerJ 1: DOI: 10.7717/peerj.21

[5] Bell, R.A.V, and Storey, K.B. (2012). Regulation of liver glutamate dehydrogenase from an anoxia tolerant freshwater turtle. HOAJ Biol. 1: 3

[6] Bell, R.A.V., Dawson, N.J., and Storey, K.B. (2012). Insights into the in vivo regulation of glutamate dehydrogenase from the foot muscle of an estivating land snail. Enz. Res. Article ID 317314.

[7] Bell, R.A.V. and Storey, K.B. (2010). Regulation of liver glutamate dehydrogenase by reversible phosphorylation in a hibernating mammal. Comp. Biochem. Physiol. B Biochem. Mol. Biol. 157: 310-316.

\section{Publications (submitted):}

[8] Bell, R.A.V., Smith, J.C. and Storey, K.B. Purification and properties of glyceraldehyde-3-phosphate dehydrogenase from the skeletal muscle of the hibernating thirteen-lined ground squirrel. J. Comp. Phys. B (under review)

[9] Bell, R.A.V. and Storey, K.B. Novel detection method for chemiluminescence derived from the Kinase-glo luminescent kinase assay platform: Advantages over traditional microplate luminometers. Methods $X$ (under review).

\section{Manuscripts (finished and prepared for publication):}

[10] Bell, R.A.V. and Storey, K.B. Regulation of skeletal muscle pyruvate kinase from the hibernating ground squirrel Spermophilus richardsonii. 
[11] Lama, J.L., Bell, R.A.V., and Storey, K.B. Glucose-6-phosphate dehydrogenase regulation in the kidney and liver of the hibernating ground squirrel, Spermophilus richardsonii.

[12] Wu, C.W., Bell, R.A.V., and Storey, K.B. Regulation of PTEN function and structural stability in hibernating thirteen-lined ground squirrels.

[13] Bell, R.A.V. and Storey, K.B. A structural comparison of glyceraldehyde-3phosphate dehydrogenase from a hibernating and non-hibernating mammal.

[14] Bell, R.A.V. and Storey, K.B. Regulation of liver and skeletal muscle fructose-1,6bisphosphatase by reversible phosphorylation within the hibernating thirteen-lined ground squirrel.

[15] Bell, R.A.V. and Storey, K.B. Purification and properties of protein phosphatase 2A from thirteen-lined ground squirrel skeletal muscle during hibernation.

[16] Bell, R.A.V. and Storey, K.B. Reversible phosphorylation and suppression of glycogen synthase kinase 3 within the skeletal muscle of hibernating thirteen-lined ground squirrels.

[17] Katzenback, B.A., Bell, R.A.V., and Storey, K.B. Entrance into hibernation is associated with a pro-inflammatory response in the liver of the thirteen-lined ground squirrel (Ictidomys tridecemlineatus). 


\section{Appendix III}

Communications at Scientific Meetings 


\section{List of Conferences (selected)}

Lama, J.L., Bell, R.A.V., Storey, K.B. Antioxidant defense in an anoxia-tolerant mollusc: The role of hexokinase and glucose-6-phosphate dehydrogenase regulation in increasing the potential for NADPH production. $8^{\text {th }}$ meeting of the Canadian Oxidative Stress Consortium, June 11-13, 2014.

Bell, R.A.V. and Storey, K.B. Posttranslational modification of glyceraldehyde-3phosphate dehydrogenase from a hibernating mammal: Insight into cold-adaptation and structural diversity of a housekeeping enzyme. ACCryo2014, Key Largo, Florida, January 15-19, 2014.

Bell, R.A.V. and Storey, K.B. Posttranslational modification of glyceraldehyde-3phosphate dehydrogenase from a hibernating mammal: Insight into cold-adaptation and structural diversity of a housekeeping enzyme. CRYO2013, 50 th Annual Meeting, Society for Cryobiology, Bethesda, Maryland, July 28-31, 2013.

Wu, C.-W., Bell, R.A.V. and Storey, K.B. Regulation of PTEN function and structural stability in hibernating thirteen-lined ground squirrels. ACCryo 2013, Miami, Florida, USA. January 2-7, 2013.

Bell, R.A.V. and Storey, K.B. Regulation of liver glutamate dehydrogenase from an

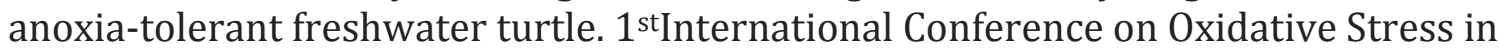
Aquatic Ecosystems. Los Cabos, Mexico, November 20-24, 2012.

Bell, R.A.V. and Storey, K.B. Posttranslational modification of glyceraldehyde-3phosphate dehydrogenase in a hibernating mammal: insight into the cold adaptation and structural diversity of a housekeeping enzyme. $19^{\text {th }}$ Methods in Protein Structure Analysis (MPSA), Ottawa University, Ottawa, Ont., Canada. June 25-28th, 2012.

Wu, C.-W., Bell, R.A.V. and Storey, K.B. Regulation of PTEN function and structural stability in hibernating thirteen-lined ground squirrels. 19 ${ }^{\text {th }}$ Methods in Protein Structure Analysis \& $2^{\text {nd }}$ China Canada Systems Biology Conference, Ottawa, ON, June 25-28, 2012.

Bell, R.A.V. and Storey, K.B. Regulation of foot muscle glutamate dehydrogenase from an estivating land snail. Oral Presentation given at the $49^{\text {th }}$ annual Canadian Society of Zoologists Conference, Ottawa University, Ottawa, Ont., Canada. May, 2011.

Bell, R.A.V. and Storey, K.B. Regulation of glutamate dehydrogenase from an anoxiatolerant freshwater turtle. Ottawa Carleton Institute of Biology Poster Day (refereed), Carleton University, Ottawa, Ont., Canada, April 2011 
Bell, R.A.V. and Storey, K.B. Regulation of foot muscle glutamate dehydrogenase from an estivating land snail. Ottawa Carleton Institute of Biology Poster Day (refereed), Carleton University, Ottawa, Ont., Canada, April 2010

Bell, R.A.V. and Storey, K.B. Regulation of glutamate dehydrogenase by reversible phosphorylation in liver of Richardson's ground squirrels. Poster presented in the $34^{\text {th }}$ Annual APICS/CIC Student Chemistry Conference, Antigonish, N.S., May $14^{\text {th }}$ $16^{\text {th }}, 2009$.

Bell, R.A.V. and Storey, K.B. Regulation of glutamate dehydrogenase from an anoxiatolerant freshwater turtle. Oral presentation given at the 12th Annual Chemistry and Biochemistry Graduate research Conference (refereed). Concordia University, Montreal, Quebec, Canada, November 23 and 24, 2009.

Bell, R.A.V. and Storey, K.B. Regulation of glutamate dehydrogenase by reversible phosphorylation in liver of Richardson's ground squirrels. Poster presented in the 10th Annual Chemistry and Biochemistry Graduate research Conference (refereed). Concordia University, Montreal, Quebec, Canada, November 23 and 24, 2007.

Bell, R.A.V. and Storey, K.B. Regulation of glutamate dehydrogenase by reversible phosphorylation in liver of Richardson's ground squirrels. Ottawa Carleton Chemistry Institute Poster Day (refereed), Carleton University, Ottawa, Ont., Canada, May 11, 2007. (Awarded Best Poster Prize) 